\title{
WIPP Waste Characterization \\ Program Sampling and Guidance Manual
}

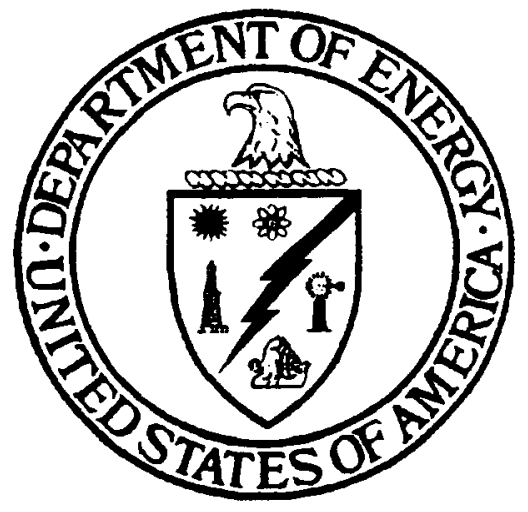

Waste Isolation Pilot Plant (WIPP)

Carlsbad, New Mexcico 
This document is issued by Westinghouse Electric Corporation, Waste Isolation Division, as the Managing and Operating Contractor for the Department of Energy, Waste Isolation Pilot Plant, Carlsbad, New Mexico, 88221.

\title{
DOE CONTRACT NUMBER: DE-AC04-86AL31950
}

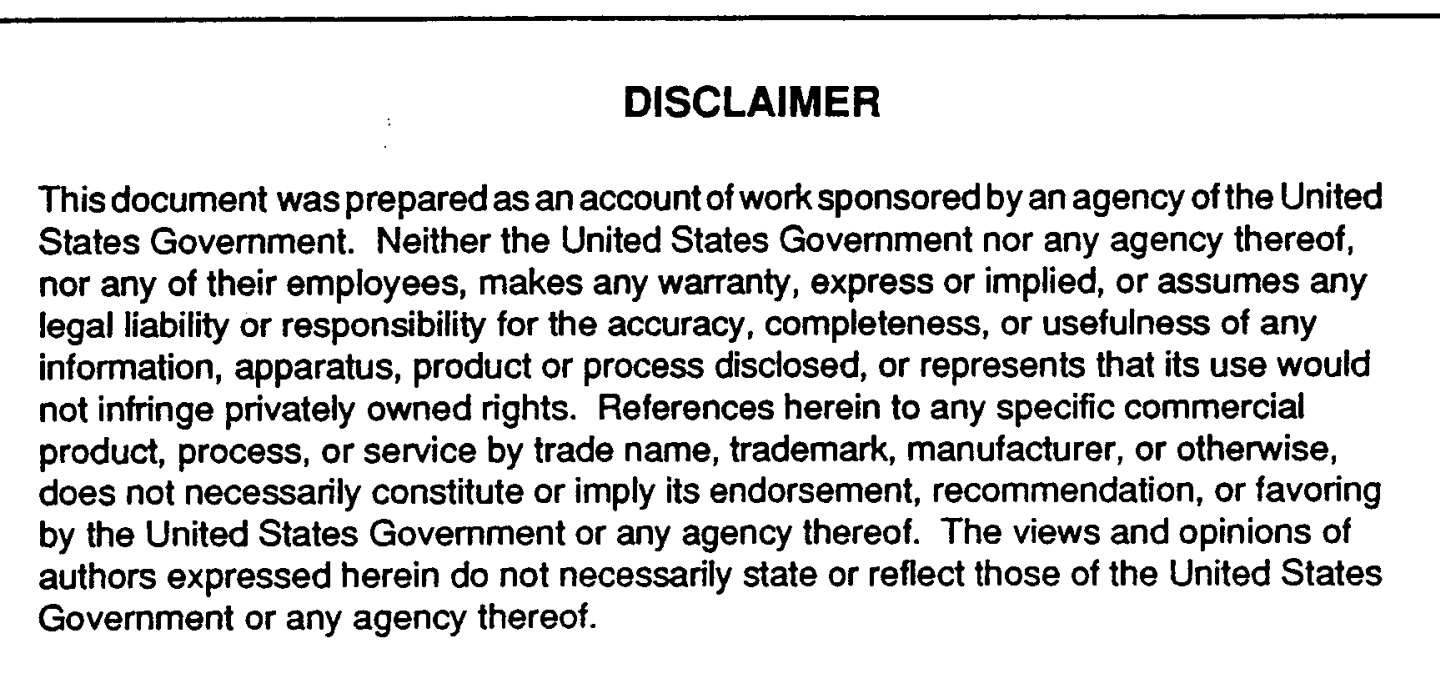

This document has been reproduced directly from the best possible copy. It is available to DOE and DOE contractors at the following address:

Office of Scientific and Technical Information

P.O. Box 62

Oak Ridge, TN 37831

Prices available from (615) 576-8401; FTS 626-8401

\author{
Available to the public from the \\ National Technical Information Service \\ U.S. Department of Commerce \\ 5285 Port Royal Road \\ Springfield, VA 22161
}

DISTRIBUTION OF THIS DOCUMENT IS UNLIMITED 


\section{DISCLAIMER}

The enclosed document has not received any secondary reviews by the U.S. Department of Energy's Office of Scientific and Technical Information (OSTI) for public releasability Post 9/11. It is being made available with the understanding that any further distribution, beyond the requesting organization, is the responsibility of the receiving organization/individual. Any distribution outside the DOE community may require additional reviews by the originating site in compliance with Secretary Abraham's May 30, 2002, memorandum titled "Safeguarding Information Pertaining to Weapons of Mass Destruction and Other Sensitive Information." 


\section{DISCLAIMER}

Portions of this document may be illegible in electronic image products. Images are produced from the best available original document. 
TABLE OF CONTENTS

INTRODUCTION

1.0 SAMPLING

110 Headspace Sampling

110.1 Headspace Gas Sampling of the 55-Gallon Poly-Bag Within a TRU Waste Drum

110.2 Headspace Gas Sampling of Innermost Layers of Confinement Within a TRU Waste Drum

110.3 Headspace Gas Sampling of WIPP Experimental Bins

2.0 CLEANING

210 Canister Cleaning

210.1 SUMMA ${ }^{\circledR}$ Passivated Stainless Steel Canister Certification and Cleaning

\subsection{PHYSICAL WASTE FORM CHARACTERIZATION}

310 Nondestructive/Destructive Characterization

310.1 Real-time Radiography Procedure

310.2 Visual Examination Procedure

\subsection{ORGANICS}

410 Sample Preparation

420 Sample Clean-up

430 Gas Chromatographic/Mass Spectrometric Volatile Organic Compound Analysis

430.1 Modified Method T0-14 for the Determination of Volatile Organic Compounds in Waste Container Headspace Using SUMMA ${ }^{\oplus}$ Passivated Canister Sampling and GCMS Analysis

430.2 Modified Method $8240 / 8260$ for the Determination of Volatile Organic Compounds in Waste Container Headspace Collected Using SUMMA ${ }^{\circledR}$ Passivated Canisters 
440 Gas Chromatographic Volatile Organic Compound Analysis

440.1 Gas Chromatographic-Flame Ionization Detector Determination of Alcohols and Ketones in Waste Container Headspace Collected Using SUMMA Passivated Canisters

\subsection{GASES}

510 Mass Spectrometric Analysis

510.1 Determination of Headspace Gases by Mass Spectrometry

520 Gas Chromatographic Analysis

520.1 Determination of Headspace Gases by Gas Chromatography

530 Spectrophotometric Methods

530.1 Determination of the Combined Nitrogen Oxide Content of Headspace Gas Samples

\subsection{INORGANICS}

\subsection{ASSAYS}

\subsection{CHARACTERISTICS AND PROPERTIES}

810 Methods for Determining Characteristics

810.1 Modified ASTM Method E 681-85 for the Evaluation of the Flammability of Waste Container Headspace Using SUMMA Passivated Canister Sampling 


\section{INTRODUCTION}

\section{PURPOSE OF THE MANUAL}

The Waste Isolation Pilot Plant (WIPP) Waste Characterization Program Sampling and Analysis Guidance Manual (Guidance Manual) provides a unified source of information on the sampling and analytical techniques that enable Department of Energy (DOE) facilities to comply with the requirements established in the current revision of the Quality Assurance Program Plan (QAPP) for the WIPP Experimental-Waste Characterization Program (the Program). This Guidance Manual includes all of the sampling and testing methodologies accepted by the WIPP Project Office (DOEMPO) for use in implementing the Program requirements specified in the QAPP. This includes methods for characterizing representative samples of transuranic (TRU) wastes at DOE generator sites with respect to the gas generation controlling variables defined in the WIPP bin-scale and alcove test plans, as well as waste container headspace gas sampling and analytical procedures to support waste characterization requirements under the WIPP test program and the Resource Conservation and Recovery Act (RCRA).

The procedures in this Guidance Manual are comprehensive and detailed and are designed to provide the necessary guidance for the preparation of site-specific procedures. The use of these procedures is intended to provide the necessary sensitivity, specificity, precision, and comparability of analyses and test results. The solutions to achieving specific program objectives will depend upon facility constraints, compliance with DOE Orders and DOE facilities' operating contractor requirements, and the knowledge and experience of the TRU waste handlers and analysts. With some analytical methods, such as gas chromatography/mass spectrometry, the Guidance Manual procedures may be used directly. With other methods, such as nondestructive/destructive characterization, the Guidance Manual provides guidance rather than a step-by-step procedure. Each DOE site must document the details of the procedures it will use and demonstrate the efficacy of such procedures to the DOEMPO prior to participation in the Program.

\section{ORGANIZATION AND FORMAT}

This Guidance Manual is issued by the DOEMWP and is not a controlled document. However, the revision number and date do appear on each page, and future revisions of this Guidance Manual will be published to reflect requirements in future QAPP revisions.

The format of this Guidance Manual is analogous to the format of Test Methods for Evaluating Solid Waste; Physical/Chemical Methods. Major categories of methods and analytes are subdivided into a series of procedures. Each method is indicated by a three-digit number and each procedure under a method is uniquely identified by the addition of a decimal identifier following the method number. For some samples, analysts may be required to select an appropriate sample preparation procedure and 
sample clean-up procedure in addition to an analytical procedure. New methods and new procedures will be added as required to ensure that all DOE sites participating in the Program have available DOE procedures for meeting program requirements.

For procedures to be included in this Guidance Manual, they must satisfy all of the programmatic requirements and quality assurance objectives identified in the QAPP. In addition, they must undergo peer review, be organized in the appropriate format, and receive approval by DOE/WPO. 


\subsection{SAMPLING}

This Guidance Manual contains the following procedures for collecting a headspace gas sample from a waste container:

Procedure 110.1 provides a procedure for the collection of representative headspace gas samples from the 55-gallon poly liner bag within a TRU waste drum. A multi-port sampling manifold, equipped with a purge assembly, is used to collect headspace samples of gases and volatile organic compounds that have a vapor phase component at room temperature and pressure. Samples are collected in SUMMA: passivated canisters.

Procedure 110.2 provides procedures for two SUMMA ${ }^{(k)}$ canister based systems for representative headspace gas sampling of innermost layers of confinement within a TRU waste drum. The first sampling system uses a low internal volume headspace gas sampling manifold; the second uses a needle and filter attached directly to the SUMMA: sample canister.

Procedure 110.3 provides a procedure for the collection of representative headspace gas samples from within the experimental bins used during the WIPP Test Phase. Headspace samples are collected in SUMMA canisters using a WIPP experimental bin headspace gas sampling system.

Analysts must consult the most current revision of the QAPP for headspace sampling quality assurance objectives, headspace sample containers and holding times, headspace sample custody procedures, and headspace sampling equipment calibration procedures and frequencies. 


\section{PROCEDURE 110.1}

\section{HEADSPACE GAS SAMPLING OF THE 55-GALLON POLY BAG WITHIN A TRU WASTE DRUM}

\subsection{SCOPE AND APPLICATION}

1.1 The sampling procedure described in this section may be used to collect representative headspace gas samples from the 55-gallon poly bag within a TRU waste drum. This sampling procedure is based on guidelines in EPA's Method TO-14 and SW-846. As such, it is applicable to sampling the headspace gases in drums containing all types of waste. The method is used to sample for gases (inorganic and hydrocarbon) and specific volatile organic compounds that have a vapor phase component at room temperature and pressure. It is based on the collection of samples in SUMMA passivated canisters. The equipment and procedures necessary to collect samples are described below.

1.2 This sampling procedure employs a multiport sampling manifold capable of collecting samples of headspace gas from within waste drums for analysis and quality control purposes. The procedure is designed for samples to be collected from within a 55-gallon poly liner bag after a waste drum's lid and internal rigid liner lid have been removed. Cleaned and evacuated sample canisters are attached to an evacuated sampling manifold. A changeable needle assembly with a valve is connected to the sampling manifold. The needle is inserted into the headspace of the poly liner bag and the valve is opened, allowing the headspace gases within the poly bag to expand into the evacuated sample canisters.

1.3 The sampling manifold is equipped with a purge assembly that allows sampling manifold blanks, field reference standards, and field duplicates to be collected through the entire manifold, from the needle tip through all of the same components that the headspace gas passes through. Field blanks may be collected through the sampling manifold, provided a sampling manifold blank is collected, or a real-time analysis of manifold cleanliness is performed with a manifold-mounted photoionization detector (PID) just before the field blank is collected. However, it is recommended that field blanks be collected in the immediate vicinity of the waste drums prior to removal of the drum lid, by opening an evacuated sample canister allowing room air to expand into the evacuated canister.

1.4 Before sampling, the total amount of available headspace gas should be estimated and compared to the combined volume of the sample canisters and sampling manifold. The available 
headspace gas should be sufficient to ensure that sampling will not withdraw more than approximately $10 \%$ of the available headspace gas.

\subsection{APPARATUS}

2.1 Collecting representative samples of headspace gas from the 55-gallon poly bag within a TRU waste drum requires the use of a variety of components. The major components required for sampling include a sampling manifold, sample canisters, pressure sensors, a thermometer, a vacuum pump, cylinders of pressurized gas, and a rotameter. In addition, the use of a PID is recommended to provide immediate indications of sampling manifold cleanliness. Each major component of the sampling apparatus is described below.

2.2 Sampling Manifold: With the exceptions of the pressurized gas cylinders, the vacuum pump and the PID, all components of the sampling manifold are designed to be panel-mounted in close configuration, minimizing the distance between components and, therefore, the system's internal volume and surface area. The panel can be mounted in a standard 19-inch rack mount cabinet on a moveable cart with the pressurized gas cylinders and vacuum pump for a mobile system, or on a wall. Figure 1 illustrates a potential configuration of the headspace sampling system. Table 1 lists the major parts required for this configuration. The numbered components in Figure 1 correspond to the numbered components described in the text below.

2.2.1 The sampling manifold is a system with two pneumatically distinct lines: an evacuated line for collecting samples and a pressurized line for providing zero air, helium or nitrogen, and field reference standard gases.

2.2.2 The evacuated line consists of a needle assembly, capacitance manometer, six ports for attaching sample canisters, and a dry vacuum pump. The internal volume, from the tip of the needle to the ports where the sample canisters attach, as shown in Figure 1, is estimated to be less than 200 milliliters. The needle consists of a 6-inch tube, 1/4-inch O.D., sharpened at one end and connected to an elbow fitting at the other (1). The elbow fitting is connected to a 0.5 micron filter (2), which is connected to the body portion of a quick-connect (3). The quick-connect allows the operator to easily connect a clean needle assembly between samples, thus reducing the potential for cross contamination.

2.2.3 A flow regulating bellows valve $(4)$ is connected to the stem side of the quickconnect. On the other side of valve $(4)$ is a 7 -micron filter $(6)$ which provides additional protection 
Revision: 0

Date: June 14, 1991

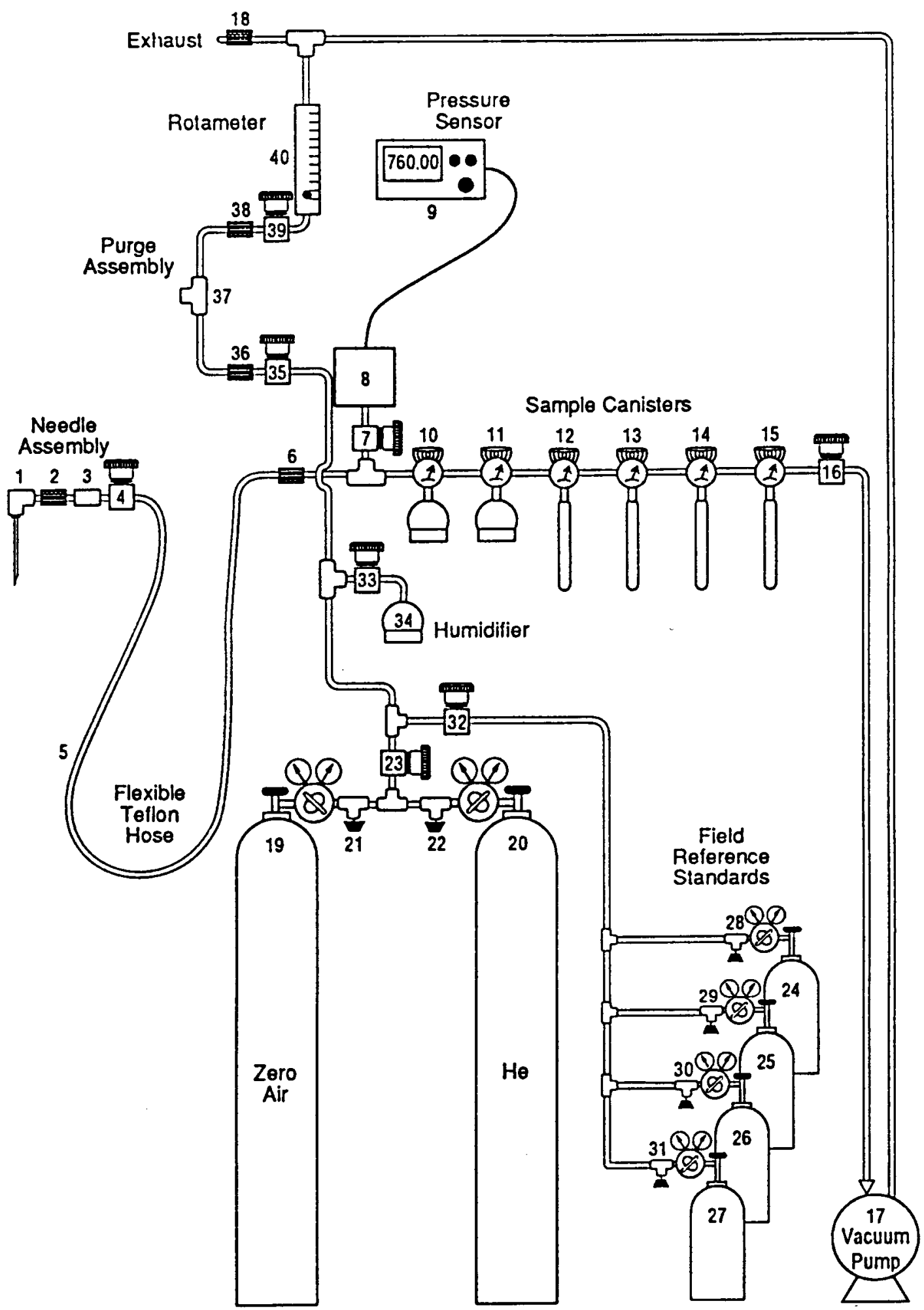

Figure 1. Configuration of the 55-Gallon Poly Bag Headspace Gas Sampling Manifold 
Table 1. 55-Gallon Poly Bag Headspace Gas Sampling System: Listing of Major Components

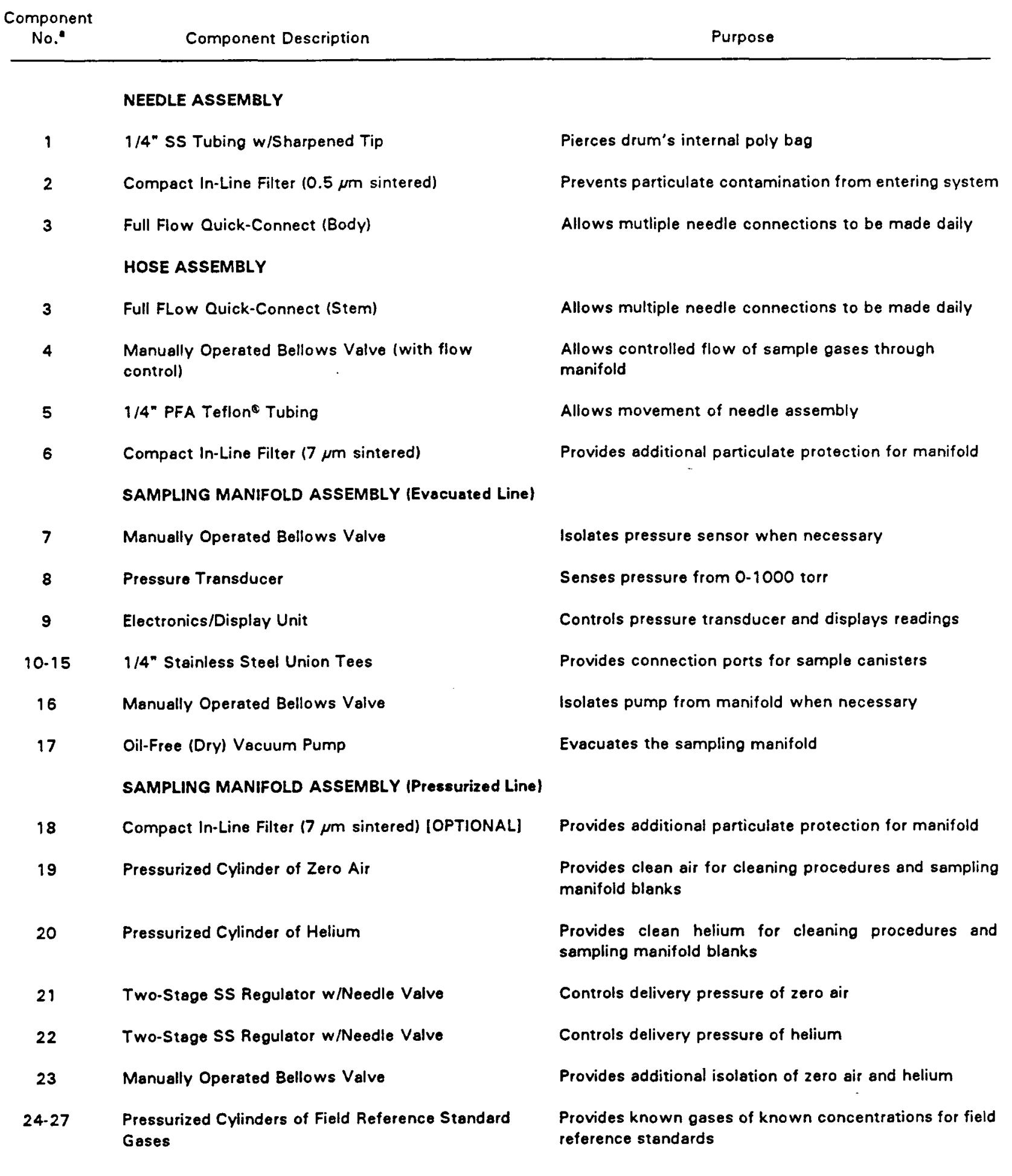


Table 1. 55-Gallon Poly Bag Headspace Gas Sampling System: Listing of Major Components (Continued)

\section{Component \\ No.} Component Description

Purpose
28-31

Two-Stage SS Regulator w/Needle Valve

32

33

34

35

36

37

38

39

40

Manually Operated Bellows Valve

Manually Operated Bellows Valve Water Control)

36

$1 / 4^{n}$ Union Tee

Manually Operated Bellows Valve

High-Accuracy Rotameter (.2-14 L/min)
$250 \mathrm{ml}$ SUMMA Canister Containing $100 \mathrm{ml}$ of

Menually Operated Bellows Vaive (with Flow

Compact In-Line filter (7 $\mu \mathrm{m}$ sintered) [OPTIONAL]

Compect In-Line Filter (7 $\mu \mathrm{m}$ sintered) [OPTIONAL]

ADDITIONAL SYSTEM COMPONENTS ${ }^{b}$

Blank Aluminum Panel $\left(19^{\prime \prime} \times 21^{\prime \prime} \times 12^{\prime \prime}\right)$

Full Range Fortin Mercury Barometer

Certified Mercury Thermometer

Portable Organic Vapor Analyzer with

Photoionization Detector (PID)

Resistive (Heat) Tape or Wire

\section{SYSTEM CLEANING ASSEMBLY}

Port Connector $\left(1 / 4^{n}\right)$

Union Elbow (1/4")

Full Flow Quick-Connect (Body)
Controls delivery pressure of field reference standards

Provides additional isolation of field reference standards Isolates humidifier from manifold when necessary

Humidifies pressurized gases

Controls flowrate of pressurized gases entering purge assembly

Provides additional particulate protection for manifold

Allows evacuated line to be connected to pressurized side

Provides additional particulate protection for manifold

Allows pressurized line cleaning after field reference standard collection

Allows system operator to monitor excess flow

Allows system components to be rack mounted

Provides ambient pressure measurements

Provides ambient temperature measurements

Provides immediate analysis for manifold cleanliness

Reduces potential for analytes to adhere to internal surfaces of manifold

Provides tight connection to purge assembly tee (37)

Connects port connector to quick-connect at 90 degrees

Allows connection to evacuated line of manifold

- See numbered components of Figure 1

- Components not shown in Figure 1 
against system contamination from particulate matter. Connecting valve $(4)$ and filter $(6)$ is flexible Teflon tubing (5) that allows movement of the needle from the purge assembly tee (37) to the 55gallon poly bag.

2.2.4 After passing through filter (6), headspace gases pass through a tee connector before being swept past the sample canister ports and into the vacuum pump. The tee provides a pneumatic link to the transducer (8) of a capacitance manometer pressure sensor. Closing bellows valve (7) protects the sensor from pressure shocks and overpressurization. CAUTION: The transducer should not be exposed to pressure greater than $1,200 \mathrm{~mm} \mathrm{Hg}$.

2.2.5 Once downstream of the pressure transducer tee, headspace gases are swept past six tees $(10-15)$ that provide connection ports for the sample canisters. Each port should have a permanent $V C R^{\oplus}$ adaptor fitting for canister attachment to the sampling manifold to circumvent the leak problem caused by reconnecting the Swagelok ${ }^{\star}$ fitting after cleanings. If desired, valves may be placed between the ports and the canister valves to facilitate closing the ports when sample canisters are not attached.

2.2.6 Bellows valve (16) connects the vacuum pump (17) to the sampling manifold. When the pump is operating, opening valve (16) decreases the system pressure and closing the valve isolates the pump. The pump exhaust is directed through a 7 -micron filter $(18)$, which serves as a safety precaution to prevent contaminated particulate matter from accidentally entering the system through this port. Filter $(18)$ is an optional component of the sampling manifold.

2.2.7 The PID (not shown) is connected to the sample port farthest from the needle assembly (15) and is used to check sampling manifold cleanliness while the system is purged with zero air. Exhaust from the PID is directed to the vacuum pump (17) exhaust line.

2.2.8 The pressurized line consists of pressurized cylinders of zero air (19), helium or nitrogen (20), and field reference standard gases (24-27), metered through two-stage stainless steel regulators $(21,22,28-31)$ to a purge assembly where flow rates of the pressurized gases can be adjusted and monitored with rotameter $(40)$. Valve $(35)$ is a flow regulating bellows valve for regulating flows from the pressurized gas cylinders through the rotameter. Valve (39) is a bellows valve that allows the operator to clean the system after collecting a field reference standard sample. Valves (23) and (32) provide additional isolation of the field reference standard gases and the zero air and helium or nitrogen. Filters $(36)$ and $(38)$ are 7 -micron particulate filters to prevent contaminated 
particulate matter from accidentally entering the system through the purge assembly tee (37). Filters (36) and (38) are optional components of the sampling manifold.

2.2.9 Bellows valve (33) connects a $250 \mathrm{~mL}$ sample canister (34) containing approximately $100 \mathrm{~mL}$ of ASTM Type II (or equivalent) water to the pressurized line. When valve (33) is open, the water humidifies the dry pressurized gases from the pressurized gas cylinders as they sweep by on their way to the purge assembly. Closing valve (33) isolates the water from the sampling manifold. The dry pressurized gases are humidified for $Q A / O C$ purposes. For $O C$ purposes, the water vapor is expected to assist with the cleaning of the internal surfaces of the sampling manifold. For $Q A$ purposes, the water vapor is expected to condition the zero air, helium, and field reference standards so that they are comparable to the headspace gas which is expected to be humid.

2.2.10 With valve (39) open and the needle assembly attached to the purge assembly tee (37), valve (35) is used to fine tune the flow rate through the rotameter (40). The purge assembly tee is a 1/4-inch stainless steel tee with Teflon ferrules seated by a stainless steel nut at the needle connection port. The needle port has been drilled to 1/4-inch I.D. to facilitate complete insertion of the needle. The pliable Teflon ferrules permit the needle to be repeatedly withdrawn from and tightened into the port. By maintaining flow through the rotameter when purging or sampling, the technician operating the system is assured that only gases from the pressurized line of the sampling manifold enter the evacuated line, thus preventing ambient air from entering the system through the rotameter, diluting the sample(s) collected and contaminating the sampling manifold.

2.2.11 The only time it is necessary to remove the Teflon ferrules from the purge assembly tee (37) is to accommodate attachment of the system cleaning assembly after the collection of a field reference standard sample. This assembly does not have the 0.5 micron particulate filter, and a port connector is used in place of the needle assembly's 6 -inch needle. This configuration allows the pressurized line, as well as the evacuated line, of the system to be evacuated, swept, and cleaned with zero air and helium or nitrogen.

2.2.12 An additional $O C$ provision which should be incorporated into the design of the sampling manifold, but not shown in Figure 1, is the ability to heat the sampling manifold during sampling and cleaning activities. This may be accomplished by wrapping the pneumatic lines, valves, and filters with resistive wire or tape in order to heat the internal surfaces of the sampling manifold (approximately $50^{\circ} \mathrm{C}$ is recommended). Heating the sampling manifold is recommended to reduce analyte adsorption on the sampling manifold's internal surfaces. 
2.3 Sample Canister: A sample canister, as illustrated in Figure 2, is a leak-free welded stainless steel SUMMA ${ }^{*}$ pressure vessel with a $\mathrm{Cr}$-NiO-passivated interior surface, bellows valve, and optional dial pressure/vacuum gauge. All sample canisters should have VCR fittings for connection to sampling and analytical equipment. The dial pressure/vacuum gauge is not a required component if a PID is used to check for sampling manifold cleanliness prior to sampling. A dial pressure/vacuum gauge on a sample canister indicates if the canister has leaked, thereby preventing sampling manifold contamination due to exposure to a contaminated sample canister. In the absence of this canister gauge, a PID provides a real-time check of sampling manifold cleanliness, reducing the need for the sampling and analysis of manifold blanks. SUMMA ${ }^{\star}$ canisters have been used in the past for VOC sample collection and have demonstrated sample storage stability of many specific VOCs (References 1 and 3). Two sizes of canisters are used for headspace gas samples: $100 \mathrm{~mL}$ canisters, and $250 \mathrm{~mL}$ canisters.

2.4 Pressure Gauges: The headspace gas sampling system uses two types of pressure gauges: a mercury barometer to measure ambient pressure and a capacitance manometer to measure sampling manifold pressure. In addition, a dial pressure/vacuum gauge may be mounted on each sample canister to verify canister leak-tightness.

2.4.1 Mercury Barometer: The mercury barometer is a full-range Fortin barometer, or equivalent, with a range of approximately 500 to $800 \mathrm{~mm} \mathrm{Hg}$. It is kept in the sampling area. Its resolution is $0.1 \mathrm{~mm} \mathrm{Hg}$ and its calibration is traceable to National Institute of Standards and Technology (NIST) standards. A dual scale thermometer should be included on the barometer case for temperature corrections, if necessary.

2.4.2 Capacitance Manometer: The capacitance manometer is pneumatically connected to the sampling manifold, as illustrated in Figure 1. The pressure sensor consists of a pressure transducer and electronics/display unit.

2.4.2.1 The transducer is able to measure absolute pressure in the range from $0.05 \mathrm{~mm} \mathrm{Hg}$ to $1000 \mathrm{~mm} \mathrm{Hg}$. The usable resolution of the measuring range is $\pm 0.005 \mathrm{~mm} \mathrm{Hg}$ at $0.05 \mathrm{~mm} \mathrm{Hg}$. Both the pressure transducer and the electronics/display unit have the capability to be rack-mounted.

2.4.3 Dial Pressure/Vacuum Gauge: A spiral bourdon-tube type dial pressure/vacuum gauge may be mounted on each sample canister. This type of gauge is helium leak tested to $1.5 \times 10^{-7}$ 

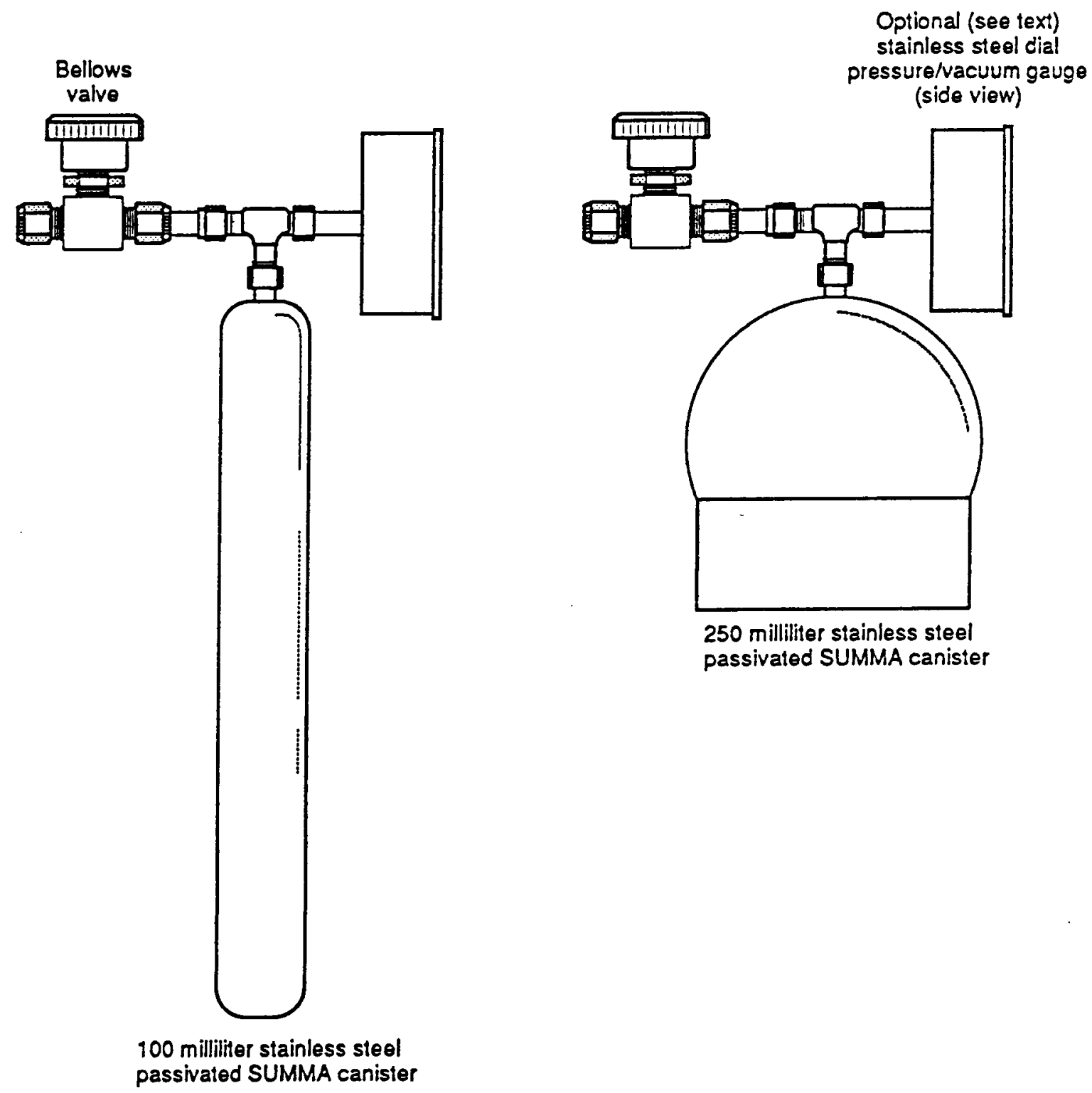

250 milliliter stainless steel passivated SUMMA canister

Figure 2. SUMMA ${ }^{\oplus}$ Canister Components Configuration (Not to Scale) 
standard $\mathrm{cc} / \mathrm{sec}$, has all stainless steel construction, and is capable of tolerating temperatures to $125^{\circ} \mathrm{C}$. The gauge is able to indicate $100 \mathrm{psig}$ pressure to a vacuum of 30 inches mercury.

2.5 Thermometer: The thermometer is a NIST-traceable laboratory grade mercury thermometer, total immersion type, with nitrogen above the mercury. It has permanent stain markings in the approximate range of $-30^{\circ}$ to $50^{\circ} \mathrm{C}$, with a resolution of $1^{\circ} \mathrm{C}$.

2.6 Vacuum Pump: The vacuum pump is a totally oil-free mechanical vacuum pump. It is able to reduce the sampling manifold pressure to $0.05 \mathrm{~mm} \mathrm{Hg}$, or lower.

2.7 Pressurized Gases: Gas from cylinders of compressed zero air and helium or nitrogen are used to clean the sampling manifold between samples and to provide gas for the collection of sampling manifold blanks. Cylinders of compressed field reference standard gases provide gas for the collection of field reference standards to assess sampling accuracy.

2.7.1 Zero Air: Zero air (certified free of hydrocarbon contamination) is supplied in steel or aluminum cylinders. It is used to clean the sampling manifold and collected as a sampling manifold blank for the VOC analyses. A $230 \mathrm{ft}^{3}$ steel cylinder is expected to provide enough zero air for 40 to 50 hours of operation.

2.7.2 Helium or Nitrogen: Helium or nitrogen is supplied in steel or aluminum cylinders. It is collected as a sampling manifold blank for the inorganic gas analyses. It is certified free of hydrocarbon contamination. One $230 \mathrm{ft}^{3}$ steel cylinder is expected to provide enough helium or nitrogen for 40 to 50 hours of operation.

\subsubsection{Field Reference Standard Gases: Field reference standard gases are supplied in} steel or aluminum cylinders. The cylinders that are used to supply field reference standard gases for VOC analyses contain certified mixtures of known VOCs at known concentrations. The cylinders that are used to supply gas for the $\mathrm{NO}_{x}$ and inorganic gas field reference standards also contain mixtures of known gases at known concentrations.

2.8 Rotameter: The glass tube rotameter, a mechanical device for indicating gas flow rate, has a polycarbonate shield and a 316 stainless steel float. Its range is from less than 1 to greater than $10 \mathrm{~L} / \mathrm{min}$, with an accuracy of \pm 2 percent. All components of the rotameter that come into contact with pressurized gases are constructed of Teflon ${ }^{*}$, glass, Viton ${ }^{\star}$, or stainless steel. The rotameter is easily dismantled for cleaning purposes. 
2.9 Photoionization Detector (PID): The PID is a battery-powered portable organic vapor analyzer capable of measuring VOCs in air in two ranges. In the range of $0-200 \mathrm{ppm}$, it has a resolution to $0.1 \mathrm{ppm}$. In the range of $200-2000 \mathrm{ppm}$, it has a resolution to $1.0 \mathrm{ppm}$. The PID uses an $11.8 \mathrm{eV}$ lamp that will detect most of the target analytes listed in Table 3-5 of the QAPP. Cylinders of zero air and $250 \mathrm{ppm}$ isobutylene are supplied by the manufacturer for calibrating the unit.

\subsection{REAGENTS AND MATERIALS}

Not applicable.

\subsection{SAMPLE HANDLING AND PROCEDURES}

4.1 This section describes the procedures that are used for cleaning the sampling manifold and procedures for collecting headspace gas samples, as well as samples for quality control purposes.

4.2 Procedures for Cleaning the Sampling System: The procedures for cleaning the sampling manifold are included to ensure sample representativeness by preventing cross-contamination between samples. These procedures maintain quality control of the sampling process. Procedure 4.2 .1 is a method for cleaning the individual system components as they are collected and assembled. This procedure also describes the steps for detecting and repairing system leaks once the system has been assembled.

Procedure 4.2.2 describes the routine system cleaning that is conducted between sampling events to prevent cross-contamination. Procedure 4.2.3 describes the cleaning conducted after the collection of a field reference standard sample. In the event Procedures 4.2.2 and 4.2.3 fail to properly clean the sampling manifold, as indicated by the sampling manifold blank and/or portable PID, the system is disassembled and cleaned as described in Procedure 4.2.1. Procedure 4.2.4 is the procedure for cleaning the needle assemblies between samples. Needle assemblies can also be disposed of after each sampling operation.

4.2.1 Initial Sampling Manifold Cleaning and Leak-Free Certification: Upon receipt from the various manufacturers and prior to assembly, all components of the sampling manifold that will come into contact with headspace or pressurized gases are inspected and thoroughly cleaned using this procedure. Parts received free of contamination, as stated or certified by the manufacturers, may be exempted from this cleaning procedure at the discretion of the sampling personnel.

1. Disassemble nonmetallic components of the sampling manifold, rinse with HPLC-grade deionized water, and dry in an oven at $50^{\circ} \mathrm{C}$ for 12 to 24 hours. An evacuated oven is preferable, but not required. 
2. Place stainless steel parts and fittings in a beaker of reagent-grade hexane in an ultrasonic bath for 15 minutes. Repeat this procedure with reagent-grade methanol. Rinse the stainless steel parts with HPLC-grade deionized water and dry in an oven at $100^{\circ} \mathrm{C}$ for 12 to 24 hours. Stainless steel parts and fittings may also be cleaned by assembling and forcing steam through the assembly.

3. Assemble the sampling manifold and leak-check by pressurizing to 10 psig and placing bubble solution on all fittings and connections. Then periodically check for the production of bubbles for a 30-minute period. As an alternative, the system may be pressurized with helium and leak-checked with a helium detection device.

4. Tighten fittings and connections that leak until no leaks are observed. From clean spare parts, replace any fittings and connections that continue to leak.

5. Evacuate the sampling manifold to $0.05 \mathrm{~mm} \mathrm{Hg}$ and monitor the pressure for 1 hour. If the pressure increases by more than $0.02 \mathrm{~mm} \mathrm{Hg}$, repeat steps 3 and 4 .

6. Purge the sampling manifold with humid zero air for 24 hours.

7. Evacuate and pressurize the sampling manifold with zero air three times.

8. Collect a sampling manifold blank through the manifold as described in Procedure 4.3.3 and analyze for the target compounds listed in Tables 3-5 and 3-6 of the QAPP. Check for system cleanliness with the PID and confirm by analysis of the sampling manifold blank.

9. Pressurize the sampling manifold with zero air to 10 psi when not in use.

\subsubsection{Routine Sampling Manifold Cleaning and Leak Check: " The following procedure} assumes that a clean needle assembly $(1-3)$ has been connected to valve $(4)$ and inserted into purge assembly tee (37). The procedure also assumes that all sampling manifold valves are closed, the vacuum pump is operating, and all regulators for the high pressure cylinders (zero air, helium or nitrogen, and field reference standards, etc.) have been properly purged and set to a delivery pressure of 10 psi.

1. Select the sample canisters to be used for sampling and, if present, check each sample canister's dial gauge vacuum readings (10-15), lightly tapping on the gauge face-plate to verify reading. Enter the reading on the sample canister tag. Attach and tighten the sample canisters to the sampling manifold. Sample canister ports not occupied by a canister must be closed with a cap or valve. If the port (to which a canister is attached) has a valve, the valve should be open prior to proceeding to step 2 .

2. Open valves (39), (23), (33), and (21).

${ }^{1}$ See Figure 3 for assistance in visualizing the process and locating the numbered components of the sampling manifold. 
Revision: 0

Date: June 14, 1991

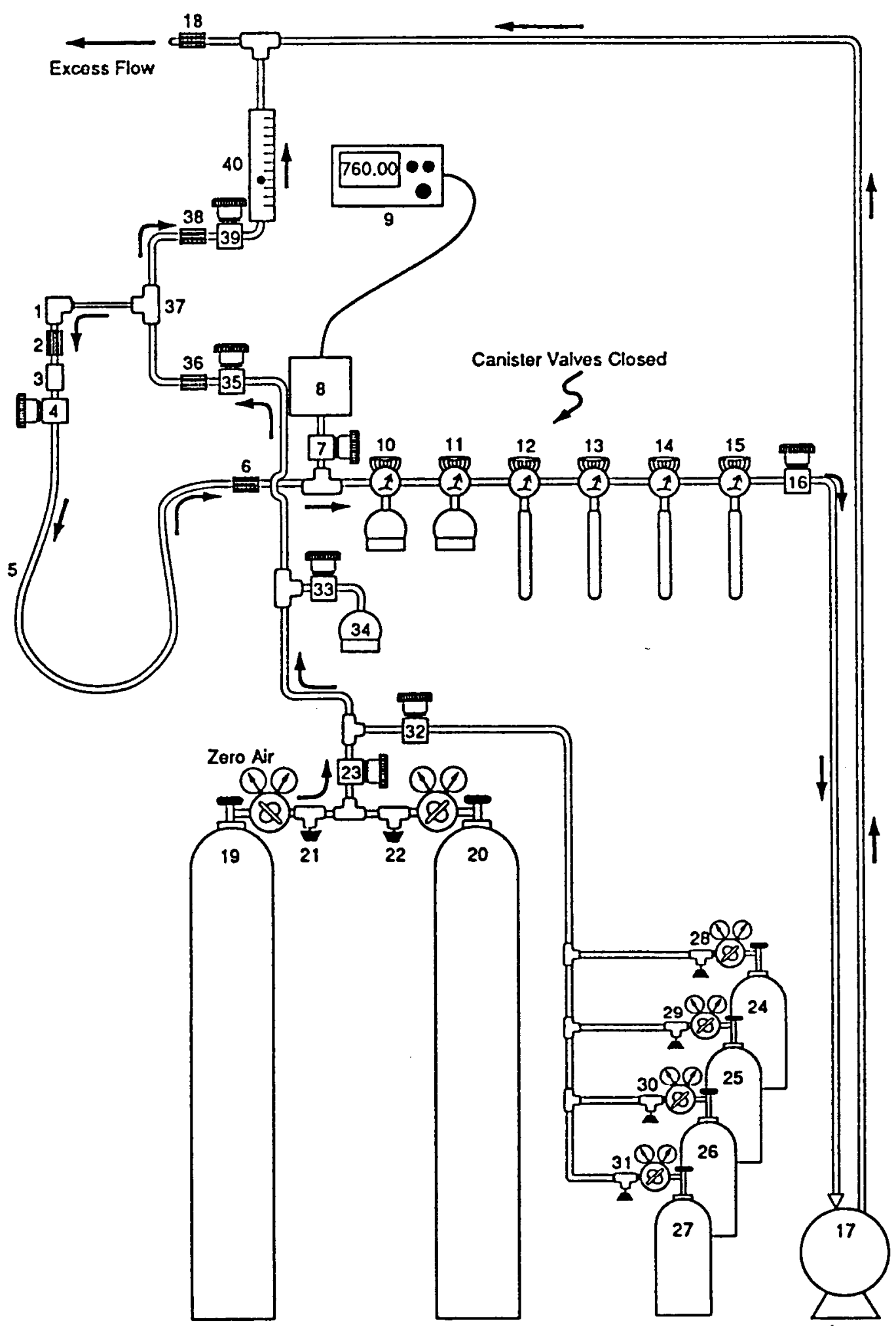

Figure 3. Sampling Manifold Configuration for Routine Cleaning and Leak Check 
3. Slowly open valve (35) until rotameter (40) indicates a flow rate of approximately 2 liters per minute.

4. Open valve (16) and slowly open valve (4) until rotameter $(40)$ indicates a flow rate of approximately 1 liter per minute. The sampling manifold is now being purged with zero air. Purge for approximately 3 minutes, periodically checking and adjusting valves (35) and (4), if necessary, to maintain rotameter flowrate at 1 liter per minute.

5. Close valve (4) to evacuate the sampling manifold. Wait 5 seconds, close valve (16), and reopen valve (4) to allow the sampling manifold to fill with zero air. Repeat this 3 to 4 times. Terminate this procedure by closing valve (16), opening valve (4), and allowing the sampling manifold to fill with zero air. If a PID is attached to a valve at canister port (15), open valve (15) and turn on the PID. Observe the PID display for at least 10 seconds. If the PID indicates a VOC concentration of $0.5 \mathrm{ppm}$ or greater, continue purging and sweeping the sampling manifold with zero air until the PID indicates less than $0.5 \mathrm{ppm}$. When the PID indicates less than $0.5 \mathrm{ppm}$, the internal volume between valve (7) and transducer (8) should be purged by opening valve (7). Care should be taken to slowly evacuate and fill the sampling manifold, since rapid fluctuations in manifold pressure may damage the manometer when valve $(7)$ is open.

6. Open valve (7) and close valve (4).

7. Slowly open valve (16) and allow the vacuum pump to reduce the pressure in the sampling manifold to $0.05 \mathrm{~mm} \mathrm{Hg}$, as indicated by the manometer (9).

8. Once the sampling manifold pressure has been reduced to $0.05 \mathrm{~mm} \mathrm{Hg}$, close valve (16).

9. Observe the manometer (9) reading for 3 to 5 seconds to verify that all sampling manifold valves have been properly closed and no observable leaks are present. If the manometer indicates a leak, cease sampling activities and leak check the manifold as described in steps 3 through 5 of Procedure 4.2.1.

10. Begin opening sample canister valves (10) through (15) one at a time, glancing up at the manometer (9) between valve openings to check for changes in pressure. If the manometer indicates an increase in pressure of less than $0.02 \mathrm{~mm} \mathrm{Hg}$, or a decrease in pressure, the sampling manifold is ready to collect a sample by opening valve (4).

An increase in manifold pressure greater than $0.02 \mathrm{~mm} \mathrm{Hg}$ indicates a canister leak, and opening the remaining sample canister valves is not advised. All open valves should be closed and the exposed sample canisters should be removed and replaced. Then the manifold should again be purged following steps 4 through 9, above.

11. If no leaks are detected, the sampling manifold is ready to collect headspace gas samples, sampling manifold blanks, and field reference standards for $V O C$ analyses. When samples are to be collected for gas or $\mathrm{NO}_{x}$ analyses, the needle assembly should be filled with helium or nitrogen, instead of zero air, between the purge assembly tee (37) and valve (4). This should be accomplished by purging the manifold with helium or nitrogen rather than zero air prior to step 6 . If the manifold is not going to be used for immediate sampling, reduce the flow rate through rotameter $(40)$ to less than 1 liter per minute (to conserve gas). 


\subsubsection{Sampling Manifold Cleaning After Field Reference Standard Collection: ${ }^{2}$ This} procedure is performed immediately after the collection of a field reference standard. It assumes that the needle assembly $(1-3)$ used for the field standard collection is still inserted into purge assembly tee (37).

1. Remove needie assembly (1-3) from purge assembly tee (37) and disconnect (3) the needle assembly from valve (4). Place the needle assembly in a collection box for items to be cleaned or dispose of it.

2. Remove the cleaning assembly from its resealable bag and connect it to valve (4).

3. Remove the nut and Teflon* ferrules from the purge assembly tee (37).

4. Attach the system cleaning assembly to the purge assembly tee $(37)$ and tighten the connection with a wrench.

5. Close valve (39) and open valves (4) and (32).

6. Observe the manometer ( 9 ) reading until the pressure ceases to increase and then close valve (23). CAUTION: Be careful not to pressurize or evacuate the manifold too quickly as this may damage the manometer.

7. Slowly open valve (16), evacuate the sampling manifold for a minimum of 5 seconds.

8. Close valve (16) and then slowly open valve (23).

9. Repeat procedures 6,7 , and 8 three more times. Terminate this step by closing valves $(16)$ and (32).

10. Close valve (4), open valve (39), and adjust valve (35) until rotameter (40) indicates approximately 1 liter per minute.

11. Proceed with the cleaning described in Procedure 4.2.2.

4.2.4 Needle Assembly Cleaning: ${ }^{3}$ This procedure describes the steps for cleaning a needle assembly after headspace gas samples or field reference standards have been collected.

1. After samples have been collected, and the sample canister valves have been closed, detach the needle assembly (1-3) at the quick-connect (3).

2. Remove the filter assembly to an area where it can safely be disassembled and cleaned.

${ }^{2}$ See Figure 4 for assistance in visualizing the process and locating the numbered components of the sampling manifold.

${ }^{3}$ See Figure 3 for assistance in locating the numbered component of the sampling manifold. 
Revision: 0

Date: June 14, 1991

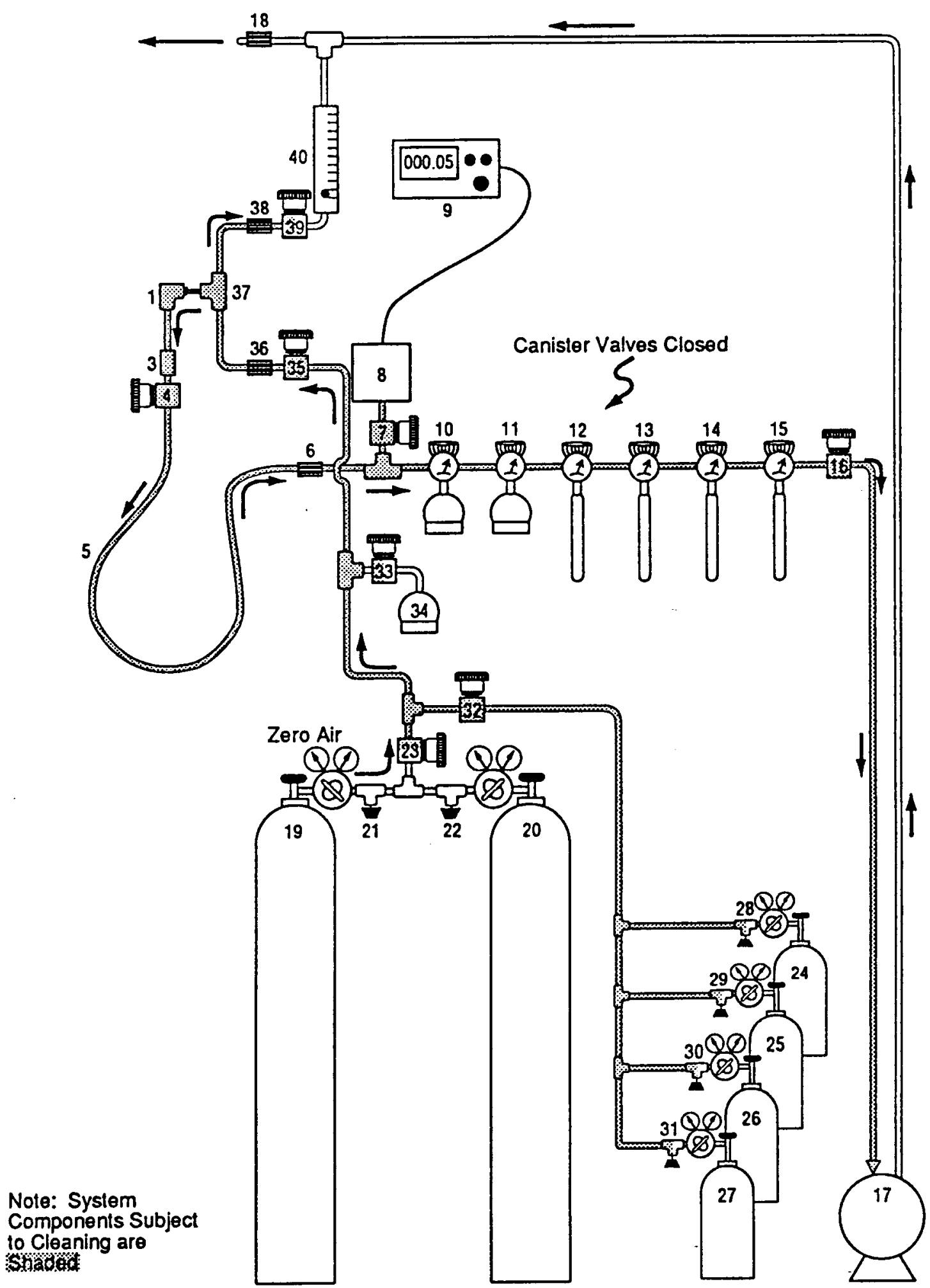

Figure 4. Sampling Manifold Configuration for Cleaning After Field Reference Standard Collection 
3. Unscrew the housing of the filter (2) and firmly grasp the filter element with a sturdy pair of forceps. Firmly pull the element to dislodge it from the housing. Properly discard the element.

4. Remove the Viton O-ring from the body of the quick-connect $(3)$ and discard the O-ring.

5. Place the reusable parts in a beaker of reagent-grade hexane in an ultrasonic bath for 15 minutes. Repeat this procedure with reagent-grade methanol. Rinse the parts with HPLCgrade deionized water and dry in an oven at $100^{\circ} \mathrm{C}$ for $12-24$ hours.

6. Insert a new filter element into the filter (2) and tightly screw the body together.

7. Place a new Viton ${ }^{0}$-ring on the body of the quick-connect (3).

8. Purge the needle assembly and a resealable bag with zero air as the needle assembly is being placed inside the bag.

9. Close the resealable bag and place it in a box for storage.

4.3 Procedures for Collecting Headspace Gas and Quality Control Samples: Headspace gas and quality control samples collected using the sampling manifold and procedures described here comply with the headspace gas sampling data quality and quality assurance objectives (see QAPP Sections 1.4 and 3.3). (Note: Prior to sampling, drums are allowed to equilibrate to the temperature of the sampling area for 72 hours.]

\subsubsection{Headspace Gas Sample Collection ${ }^{4}$}

1. Verify that the sampling manifold has been properly cleaned and leak-checked as described in Procedure 4.2.2.

2. Prepare the drum for sampling by removing the drum lid and the internal rigid liner lid which should expose the 55-gallon poly liner bag. CAUTION: Appropriate site-specific radiological controls must be established prior to removing the drum lid.

3. Locate an area within the poly bag from which a representative headspace gas sample can be withdrawn. Care should be taken to prevent placing the tip of the needle in an area where solid material or particulate matter might fill or clog the needle.

4. Remove the needle assembly (1-3) from the purge assembly tee (37) and press the needle through the poly bag and into the internal headspace. Disturbing the bag should be avoided, if possible. However, if handling the bag is necessary, the operator should do so in a manner that minimizes the potential for introduction of outside air into the poly bag's internal headspace.

5. Hold the needle in the internal headspace and open valve (4). Hold the needle steady until the manometer (9) indicates ambient pressure, or ceases to show an increase in pressure. Verify that the canister gauges, if present, indicate 0 psig.

${ }^{4}$ See Figure 5 for assistance in locating the numbered components of the sampling manifold. 
Revision: 0

Date: June 14, 1991

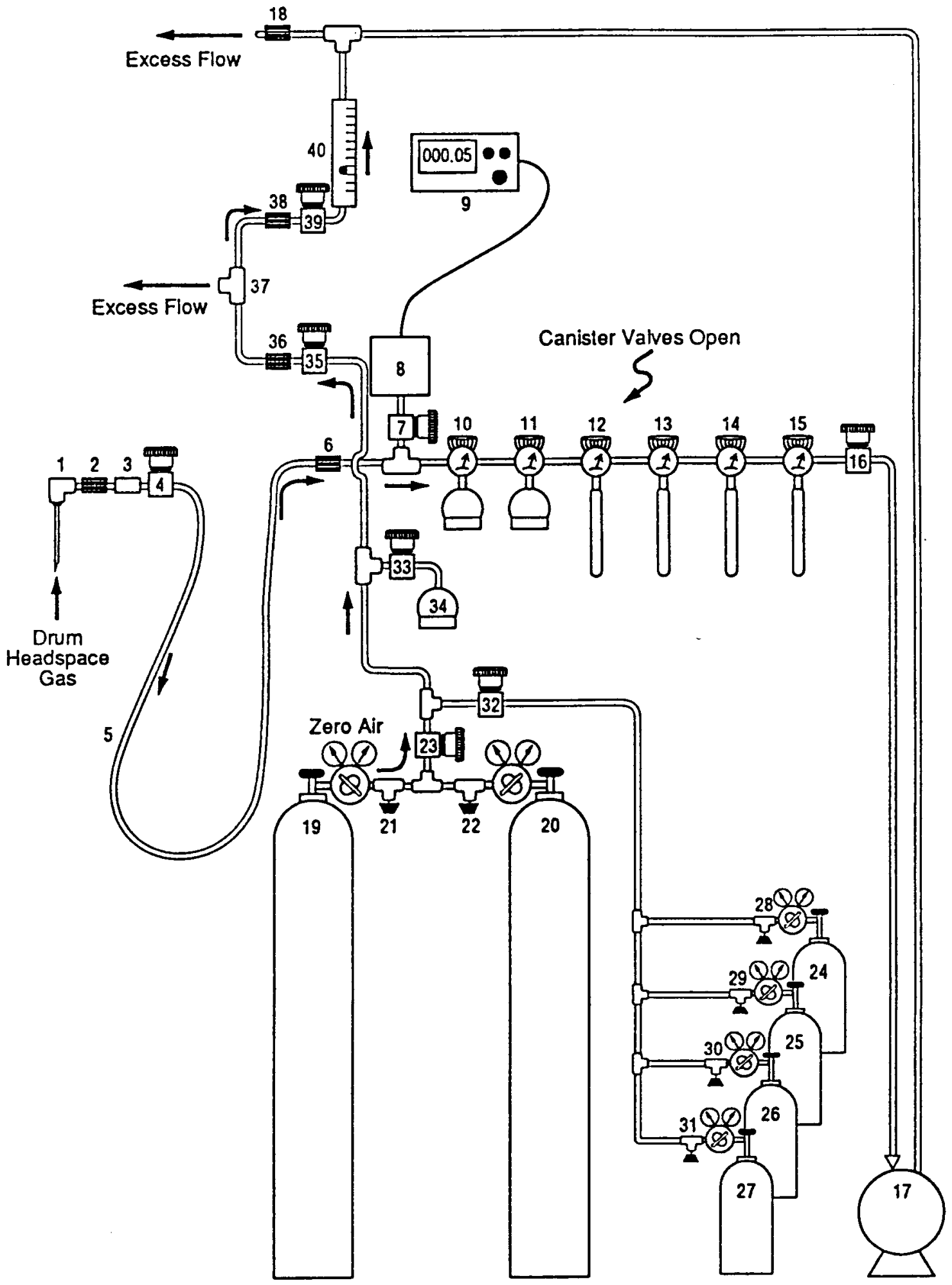

Figure 5. Sampling Manifold Configuration for Headspace Gas Sample Collection 
6. Close valves (10) through (15).

7. Remove the needle (1) from the poly bag and disconnect the needle assembly from valve (4). Place the needle assembly in a collection box for items to be cleaned or dispose of it.

8. Remove a fresh needle assembly from its resealable bag and connect it to valve (4). Insert the fresh needle assembly into purge assembly tee (37) and hand tighten the assembly in place.

9. Remove the sample canisters from the sampling manifold and log the date, time, manifold pressure (9), ambient pressure, and ambient temperature on the sample canister tags (see QAPP Section 5, Sample Canister Tagl.

10. Note the type of sample (e.g., duplicate, sampling manifold blank, etc.) that the sample canister contains on the OC sample record (see QAPP Section 5, OC Sample Record Form) and place the sample canisters in the shipping container.

11. Repeat Procedure 4.2.2.

4.3.2 Field Blank Collection: A field blank must be collected immediately prior to the collection of a headspace sample with the QC sample collection frequency specified in the QAPP, Section 9.0. Field blanks may be collected in one of two ways. The recommended procedure for collecting a field blank is to collect the sample directly into the sample canister without going through the sampling manifold, as described below. Sampling sites unable to use this procedure may collect field blanks through the sampling manifold provided they collect a sampling manifold blank immediately prior to collecting the field blank. Also prior to field blank collection, sampling sites may wish to use a PID to obtain a real-time indication of sampling manifold cleanliness.

1. Before the lid of the waste drum has been removed, place a sample canister near the top of the drum and open the sample canister valve.

2. Allow the sample canister to equilibrate to ambient pressure, close the canister valve, and log the date, time, ambient pressure, and ambient temperature on the canister tag (see QAPP Section 5, Sample Canister Tag).

3. Note that the sample canister contains a field blank on the OC sample record (see QAPP Section 5, OC Sample Record Forml and place the sample canister in the shipping container.

\subsubsection{Sampling Manifold Blank Collection ${ }^{5}$}

1. Verify that the sampling manifold has been properly cleaned and leak-checked as described in Procedure 4.2.2.

${ }^{5}$ See Figure 6 for assistance in locating the numbered components of the sampling manifold. 
Revision: 0

Date: June 14, 1991

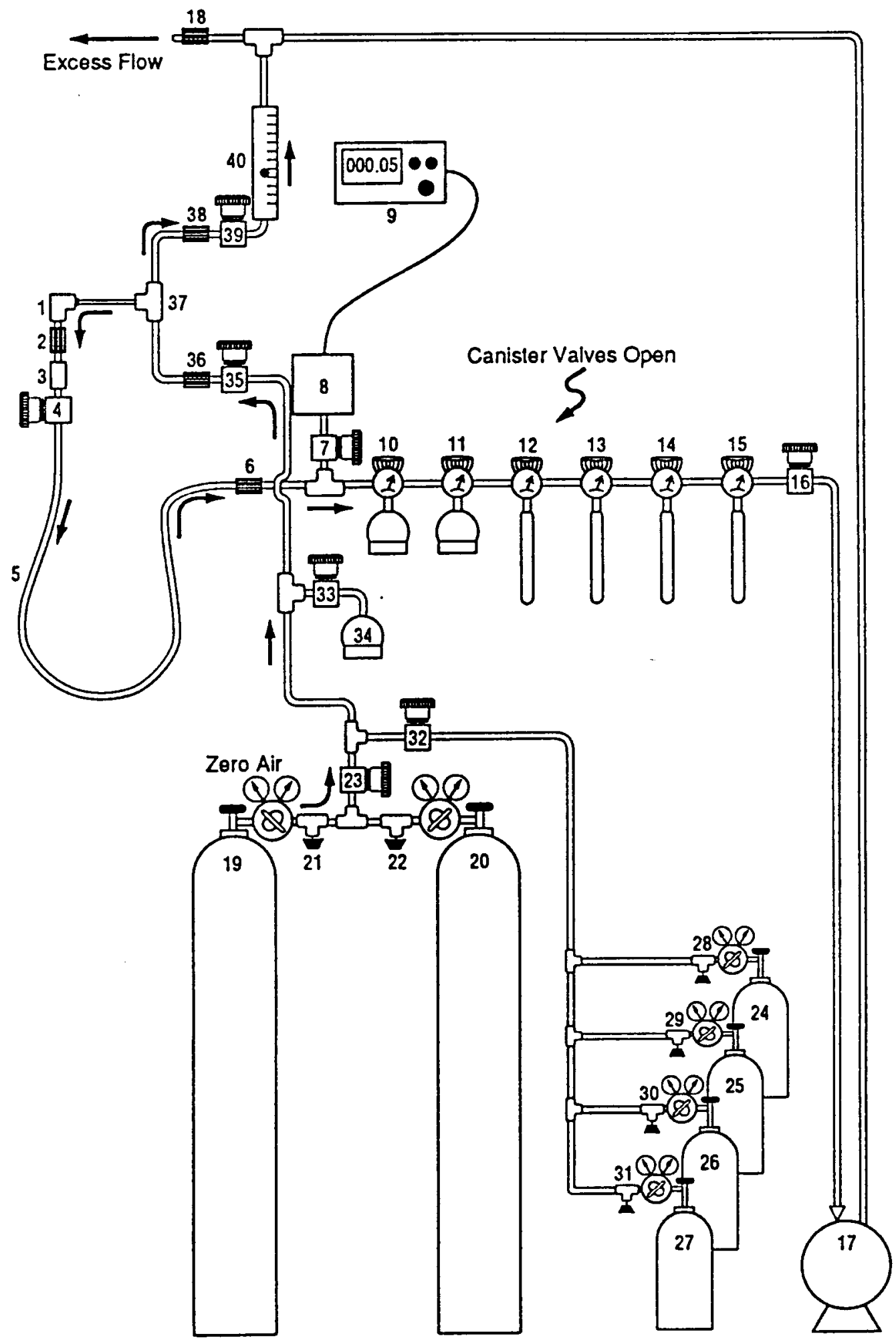

Figure 6. Sampling Manifold Configuration for Sampling Manifold Blank Collection 


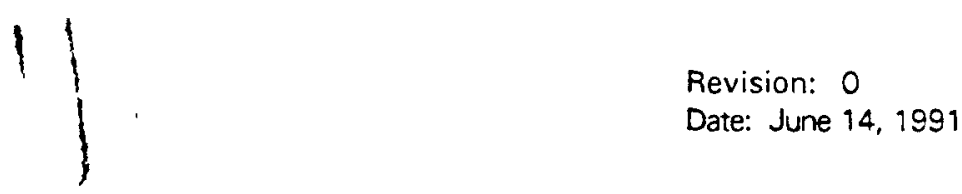

2. With the needle assembly (1-3) connected to the purge assembly tee (37), adjust valve (35) until rotameter $(40)$ indicates a flow rate of approximately 10 liters per minute.

3. Open valve (4) at a rate that allows the sample canister(s) to be filled as quickly as possible without reducing the flow indicated on the rotameter to zero (40). The rotameter should indicate excess flow at all times during collection of the sampling manifold blank.

If rotameter $(40)$ is observed to fall to zero during sampling, the sampling manifold blank should be invalidated and the sampling manifold should be cleaned (Procedure 4.2.2) prior to attempting collection of another sampling manifold blank.

4. Continue drawing zero air from the purge assembly tee (37) until the manometer (9) indicates ambient pressure or ceases to show an increase in pressure. Verify that the sample canister gauge(s), if present, indicate 0 psig.

5. Close valve (4) and valves (10) through (15) when the manometer indicates ambient pressure.

6. Adjust valve $(35)$ to reduce the flow rate through the rotameter $(40)$ to less than 1 liter per minute (to conserve zero air).

7. Remove the sample canister(s) from the manifold and log the date, time, manifold pressure (9), ambient pressure, and ambient temperature on the sample canister tags (see Section 5, Sample Canister Tagl.

8. Note that the sample canister contains a sampling manifold blank on the OC sample record (see Section 5, Sample Record Form) and place the canisters in the shipping container.

\subsubsection{Field Reference Standard Collection ${ }^{6}$}

1. Verify that the sampling manifold has been properly cleaned and leak-checked as described in Procedure 4.2.2.

2. Close valve (23), open valve (32), and open the valve to the cylinder of standard gas from which a standard sample is desired: (28) through (31). Allow standard gas to flow through the purge assembly for 2-3 minutes, opening and closing valve (35) a few times to facilitate purging residual zero air from pneumatic lines.

3. With the needle assembly (1-3) connected to the purge assembly tee (37), adjust valve (35) until rotameter $(40)$ indicates a flow rate of approximately 10 liters per minute.

4. Open valve (4) at a rate that allows the sample canister(s) to be filled as quickly as possible without reducing the flow indicated on the rotameter to zero (40). The rotameter should indicate excess flow at all times during collection of the field reference standards.

${ }^{6}$ See Figure 7 for assistance in locating the numbered components of the sampling manifold. 
Revision: 0

Date: June 14, 1991

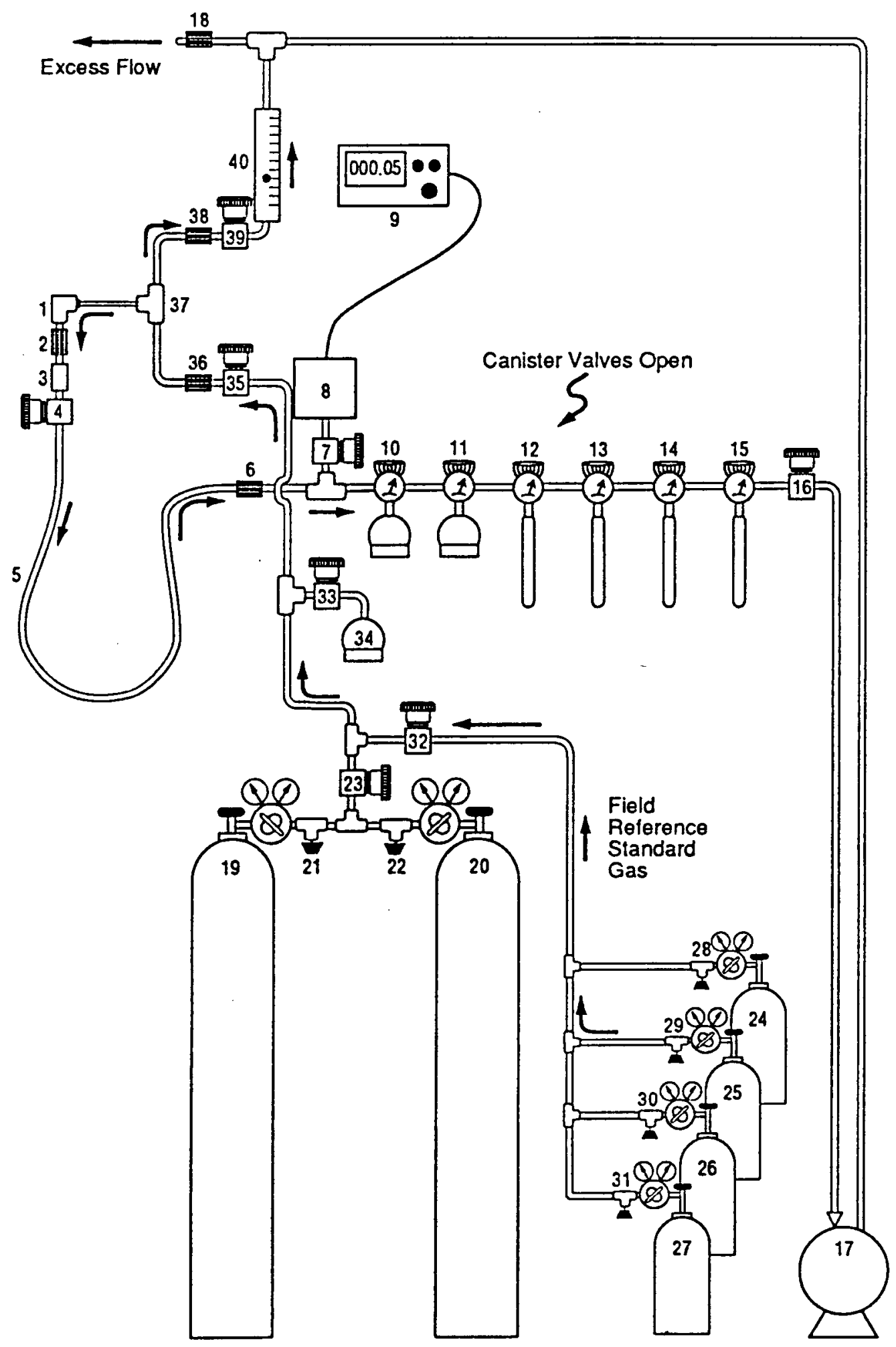

Figure 7. Sampling Manifold Configuration for Field Reference Standard Collection 
If rotameter $(40)$ is observed to fall to zero during sampling, the field reference standard should be invalidated and the sampling manifold should be cleaned (Procedure 4.2.2) prior to attempting collection of another field reference standards sample.

5. Continue drawing field reference standard gas from the purge assembly tee (37) until the manometer (9) indicates ambient pressure or ceases to show an increase in pressure. Verify that the sample canister(s) gauge(s), if present, indicate 0 psig.

6. Close valve (4) and valves (10) through (15) when the manometer indicates ambient pressure.

7. Adjust valve (35) to reduce the flow rate through rotameter (40) to approximately 1 liter per minute, close valve (32), and close the valve (28-31) of the field standards cylinder that was opened.

8. Open valve (23) and open and close valve (35) a few times to facilitate purging residual field reference standards gas from the pneumatic lines. Terminate this step with valve (35) adjusted to deliver approximately 1 liter per minute zero air through rotameter (40).

9. Remove the sample canister(s) from the sampling manifold and log the date, time, pressure sensor (9) reading, ambient barometric pressure, and ambient temperature on the sample canister(s) tag(s) (see QAPP Section 5, Sample Canister Tag).

10. Note that the sample canister(s) contain a field reference standard on the OC sample record (see QAPP Section 5, OC Sample Record Form) and place the sample canister(s) in the shipping container.

11. Cap sample canister(s) port(s) (10) through (15) and proceed with cleaning the sampling manifold as described in Procedure 4.2.3.

\subsection{CALCULATIONS}

5.1 The potential exists for sampling or analytical personnel, upon receipt of a sample canister, to find that the dial pressure gauge, if present on the sample canister, does not indicate the same pressure as that which was last logged on the sample canister tag. Changes in the sample canister gauge pressure may be the result of four possible occurrences: (1) a leak in the sample canister or sample canister component (value, gauge, etc.); (2) damage to the sample canister gauge during storage or transportation; (3) an ambient pressure difference between the sampling and analysis location; and (4) an ambient temperature difference between the sampling and analysis location.

5.2 The procedures in Section 5.3, below, should be followed to determine the sample canister gauge pressure change expected if ambient pressure and temperature differences exist between the sampling and analysis locations. If the change in sample canister gauge pressure can be accounted for using the procedures in Section 5.3, then the integrity of the sample has been maintained. If, 
however, the sample canister gauge pressure change cannot be accounted for by considering ambient pressure and temperature changes, the canister must be leak checked and re-certified for use.

\subsection{Calculations to Compute Expected Sample Canister Gauge Pressure Changes}

5.3.1 Temperature Change at Constant Ambient Pressure: Sample canister gauge pressure changes resulting from a temperature change can be explained by Equation (1):

$$
P_{2}=\frac{P_{1} T_{2}}{T_{1}}
$$

where

$P_{1}=$ is the sample canister internal pressure (ambient pressure plus gauge pressure in inches of mercuryl at the time of sampling,

$P_{2}=$ is the sample canister internal pressure lambient pressure plus gauge pressure in inches of mercuryl at the time of analysis, if the ambient temperature changes from $T_{1}$ to $T_{2}$ at constant ambient pressure.

$T_{1} \quad$ is the ambient temperature (degrees Kelvin) at the time of sampling,

$\mathrm{T}_{2}$ is the ambient temperature (degrees Kelvin) at the time of analysis.

According to this equation, increasing the temperature of the canister $\left(T_{2}>T_{1}\right)$ will increase the internal canister pressure, while decreasing the canister temperature $\left(T_{2}<T_{1}\right)$ will decrease the internal canister pressure.

\subsubsection{Ambient Pressure Change at Constant Temperature}

5.3.2.1 A sample canister's dial gauge is expected to indicate a change in pressure if the canister is transported from a high elevation to a lower elevation, or vice versa. The expected change in canister gauge pressure should be the same as the difference in ambient pressure between the high elevation site and the low elevation site, or vice versa.

5.3.2.2 Changes in sample canister gauge pressure that result from changes in temperature and ambient pressure are additive. Care, however, must be taken to ensure that the sign (positive or negative) of the calculated gauge pressure changes are accurately assigned. 


\subsection{ANALYTICAL PROCEDURE}

The analytical procedures for analysis of VOCs and gases in the headspace of waste containers are provided as Procedures 430 and 440 for VOCs, and 510, 520, and 530 for gases.

\subsection{QUALITY CONTROL FOR HEADSPACE SAMPLES}

7.1 The accuracy and precision of the headspace sampling and analysis operations are assessed by analyzing field $\mathrm{OC}$ samples and by cleaning and leak checking the sampling manifold prior to use. The field $O C$ samples include a sampling manifold blank, a field reference standard, field blank, and field duplicate. The sampling manifold blank is a sample of the zero air, helium or nitrogen used to purge the sampling manifold. The field reference standard is a sample of a high purity gas containing known concentrations of target analytes. Field blanks are samples of room air collected near the drum being sampled. Finally the field duplicates are headspace samples collected at the same time from the same 55-gallon poly bag.

7.2 The precision of the headspace sampling and analysis operation is assessed by collecting field duplicates for VOCs, gas, and $\mathrm{NO}_{x}$ analysis. The accuracy of the headspace sampling and analysis operation is assessed by cleaning and leak checking the manifold and by collecting field reference standards. The sampling manifold is thoroughly cleaned and evacuated prior to use.

7.3 Specific sampling steps to ensure the representativeness of headspace gas samples include:

- Sample canister cleaning and leak check

- Sampling manifold cleaning

- Use of sample canisters with a passivated internal surfaces

- Use of a low internal volume sampling manifold

- Small sample volume: low sample volume to available headspace gas volume ratio

- Careful pressure regulation in sample canister

- Performance audits

- Collection of sampling manifold blanks, field reference standards, field blanks, and field duplicates.

\subsection{Quality Control for Pressure and Temperature Measurements}

7.4.1 Calibration requirements described in this section pertain to the calibration of equipment and instrumentation used for temperature and pressure measurements. All information relat- 
ing to the calibrations of the pressure and temperature sensors is recorded. Prior to its use for this program, all the equipment described below is calibrated and recertified.

7.4.2 Certification Check of Mercurv Barometer: The mercury barometer is certified by the manufacturer using standards traceable to NIST. The pressure indication of the barometer is corrected in accordance with temperature indicated by a dual-scale thermometer attached to its casing, if necessary.

7.4.3 Calibration of the Capacitance Manometer: The capacitance manometer is certified using standards traceable to NIST.

7.4.4 Calibration of Sample Canister Pressure Gauge: The dial pressure gauge of each sample canister is calibrated to the laboratory pressure gauge. Three points are checked: (1) ambient, (2) full vacuum, and (3) a point midway between full vacuum and ambient pressure to be chosen by the testing technician. Adjustments are made and noted, if necessary, and the information is logged. Each canister gauge is recertified once per month, or as necessary.

7.4.5 Calibration of the Mercury Thermometer: The mercury thermometer is certified using standards traceable to NIST.

7.4.6 Calibration of the PID: The PID is calibrated once per sampling day, or as often as necessary to maintain its operation within manufacturers' specifications. Calibration gas, supplied by the manufacturer, is certified $\pm 2 \%$ of the indicated concentration. Zero air, also supplied by the manufacturer, is certified $99.9 \%$ free of hydrocarbon contamination. Additional QA/OC checks are periodically performed by obtaining and logging the PID's response to the field reference standard gases.

\subsection{Sample Canister Cleaning}

7.6.1 Sample canisters used with these procedures must undergo a rigorous cleaning in accordance with the requirements specified in Section 7.4.1 of the QAPP using the procedure in Section 210.1 of the Guidance Manual. 


\subsection{REFERENCES}

1. U.S. Environmental Protection Agency (U.S. EPA), 1988, Compendium Method TO-14, The Determination of Volatile Organic Compounds (VOC) in Ambient Air Using SUMMA Passivated Canister Sampling and Gas Chromatographic Analyses, May 1988, Quality Assurance Division, Environmental Monitoring Systems Laboratory, Research Triangle Park, North Carolina.

2. U.S. EPA, 1986, "Test Methods for Evaluating Solid Waste, Physical/Chemical Methods," SW-846, Third Edition, Office of Solid Waste and Emergency Response, Washington, D.C.

3. Oliver, K.D., J.D. Pleil, and W.A. McClenny, 1986, Atmospheric Environment, Vol. 20, No. 7, pp. 1403-1411. 


\section{PROCEDURE 110.2}

\section{HEADSPACE GAS SAMPLING OF INNERMOST LAYERS OF CONFINEMENT WITHIN A TRU WASTE DRUM}

\subsection{SCOPE AND APPLICATION}

1.1 The TRU wastes to be sent to the WIPP as part of the Test Phase are packaged in 55gallon drums at the generator storage site(s) prior to shipment and can contain waste items, residual materials, and/or packaging materials confined by several layers of plastic bags. Given this waste packaging heterogeneity, it is difficult to argue that the headspace gas sample collected from the 55gallon poly bag is truly representative of the headspace gas contributions from all the small bags within the 55-gallon bag. To determine whether the headspace gas sample collected is representative, all innermost layers of confinement in a drum are to be sampled to assess the magnitude of compositional variations that might exist.

1.2 The question of whether headspace gas sampling of waste drums is representative of the entire headspace within a drum can be addressed by sampling all of the innermost layers of confinement that meet the following minimum criteria:

- The innermost layer of confinement has a minimum of 1 liter of headspace gas from which a representative $100 \mathrm{ml}$ sample can be withdrawn. A $250 \mathrm{ml}$ sample is collected if there is enough available headspace gas (2.5 liter, minimum). When duplicates are collected, twice the amount of headspace gas should be available.

- Headspace gas contained within rigid innermost layers of confinement le.g., glass, metal, and rigid plastic containers) is to be sampled, but the poly bag containing the rigid container is to be sampled if a minimum of 1 liter of headspace gas is available.

Since the available headspace gas in the innermost layers of confinement is assumed to be very limited, only one headspace gas sample is collected for both inorganic and organic gas analyses. Samples are not collected for the determination of $\mathrm{NO}_{\mathrm{x}}$. Field duplicates, field blanks, sampling equipment blanks, and field reference standards (for both VOCs and gases) are all collected for quality control purposes at the frequencies specified in Tables 9-3 and 9-4 of the QAPP.

\subsection{APPARATUS}

2.1 To accomplish innermost layer of confinement headspace gas sampling, two SUMMA canister based headspace gas sampling systems have been developed. The first of these employs a 
low internal volume headspace gas sampling manifold similar to the manifold described in Procedure 110.1 for sampling a 55-gallon poly bag. The second employs a needle and filter attached directly to the SUMMA ${ }^{\oplus}$ sample canister. In each case, sample canisters are evacuated to $0.05 \mathrm{~mm} \mathrm{Hg}$ prior to sample collection.

One advantage of the low internal volume headspace gas sampling manifoid is that sample collection can be achieved through a radiological control barrier, such as a wall, preventing sampling personnel exposure and the need for decontamination of sample canisters. Another advantage is that the low internal volume manifold can be used for collecting the 55-gallon poly bag headspace gas sample. A further advantage of the low internal volume sampling manifold is that is can be evacuated from the tip of the sampling needle to the sample canister, thus minimizing the possibility of contamination by ambient air.

An advantage of the direct canister method is that it withdraws less headspace gas than the low internal volume manifold. This allows for representative sampling when available headspace gas is limited. A further advantage of the direct canister method is its flexibility. The direct canister sampling apparatus is more portable and therefore adaptable to a variety of sampling environments.

2.2 Low Internal Volume Headspace Gas Sampling Manifold: A sampling manifold similar to the manifold described in Procedure 110.1 can be constructed for innermost layer of confinement sampling. To reduce the internal volume of this manifold, the following modifications are made (see Figure 1 for location of numbered components): (1) Decrease the lengths of all pneumatic lines; (2) Use 1/8" flexible Teflon ${ }^{\oplus}$ tubing and $1 / 8^{n}$ stainless steel tubing for evacuated pneumatic lines; (3) Isolate sample canister ports other than the one or two required for innermost layer of confinement sampling by adding valve (16a). In addition, if the sample canisters are fitted with dial vacuum/pressure gauges, isolate sampling manifold components Nos. (7)-(9) during sample collection by closing valve (7). For more information on the other components of the sampling manifold see Procedure 110.1; Sections 2.2, 2.4.2, 2.6, 2.7, 2.8, and 2.9.

2.3 Direct Canister Headspace Gas Sampling Apparatus: Figure 2 illustrates the direct canister

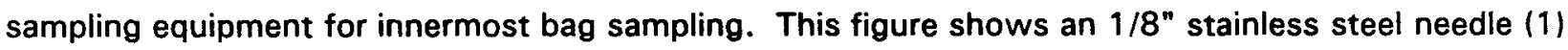
fitted via a reducer (2) to the $1 / 4$ " inlet of a 0.5 micron sintered metal filter (3). This filter is connected to the Swagelok ${ }^{\oplus}$ fitting on the SUMMA ${ }^{\oplus}$ canister $(5)$ valve via a Swagelok ${ }^{\oplus}$ to VCR $^{\oplus}$ adapter (4). The $V_{C R}{ }^{\oplus}$ fitting, with its disposable metal gasket, makes an ideal reusable connector. The combined low internal volume of these components should ensure that a representative headspace gas sample is 
Revision: 0

Date: August 30, 1991

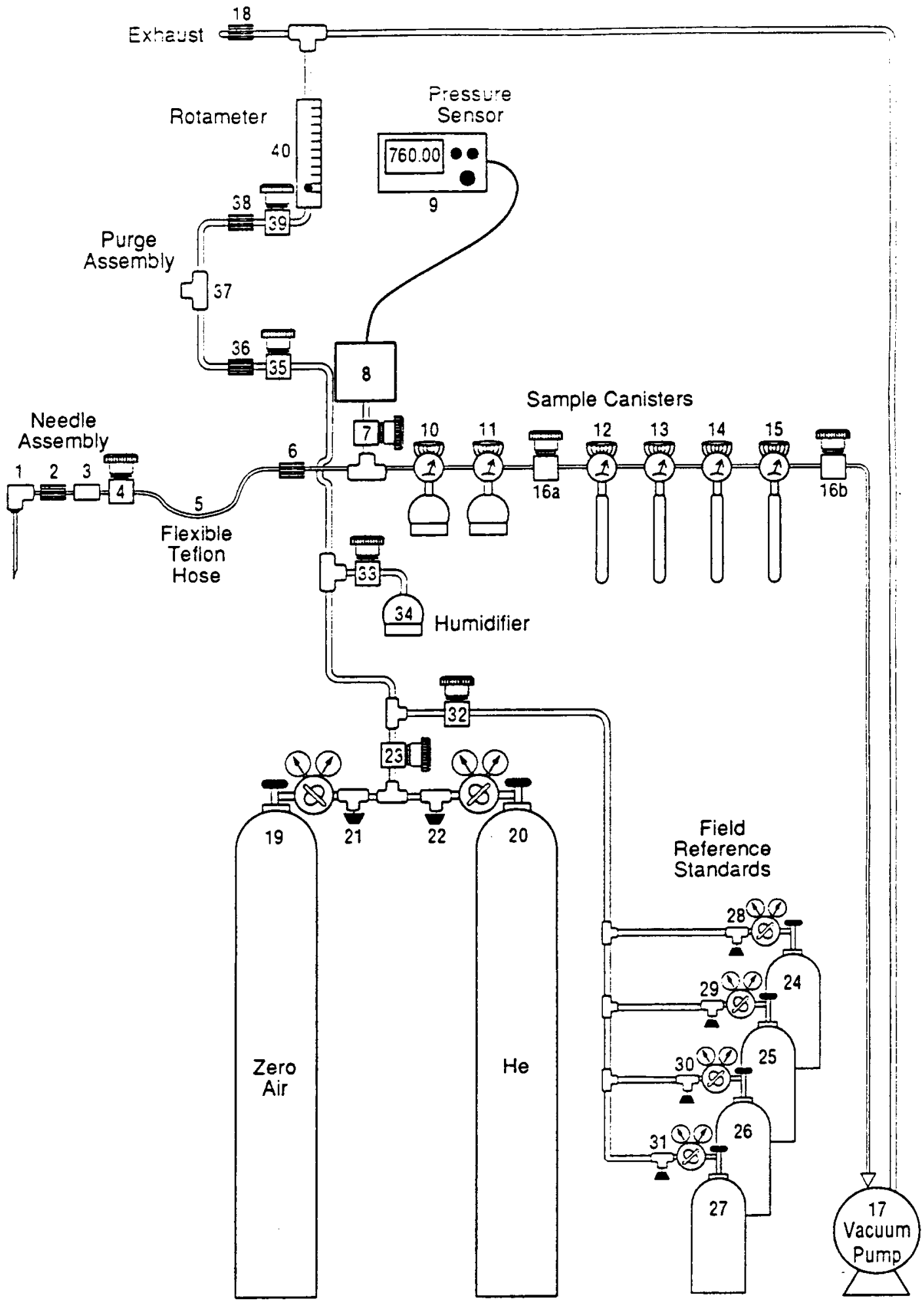

Figure 1. Low Internal Volume Sampling Manifold 
Revision: 0

Date: August 30, 1991

5

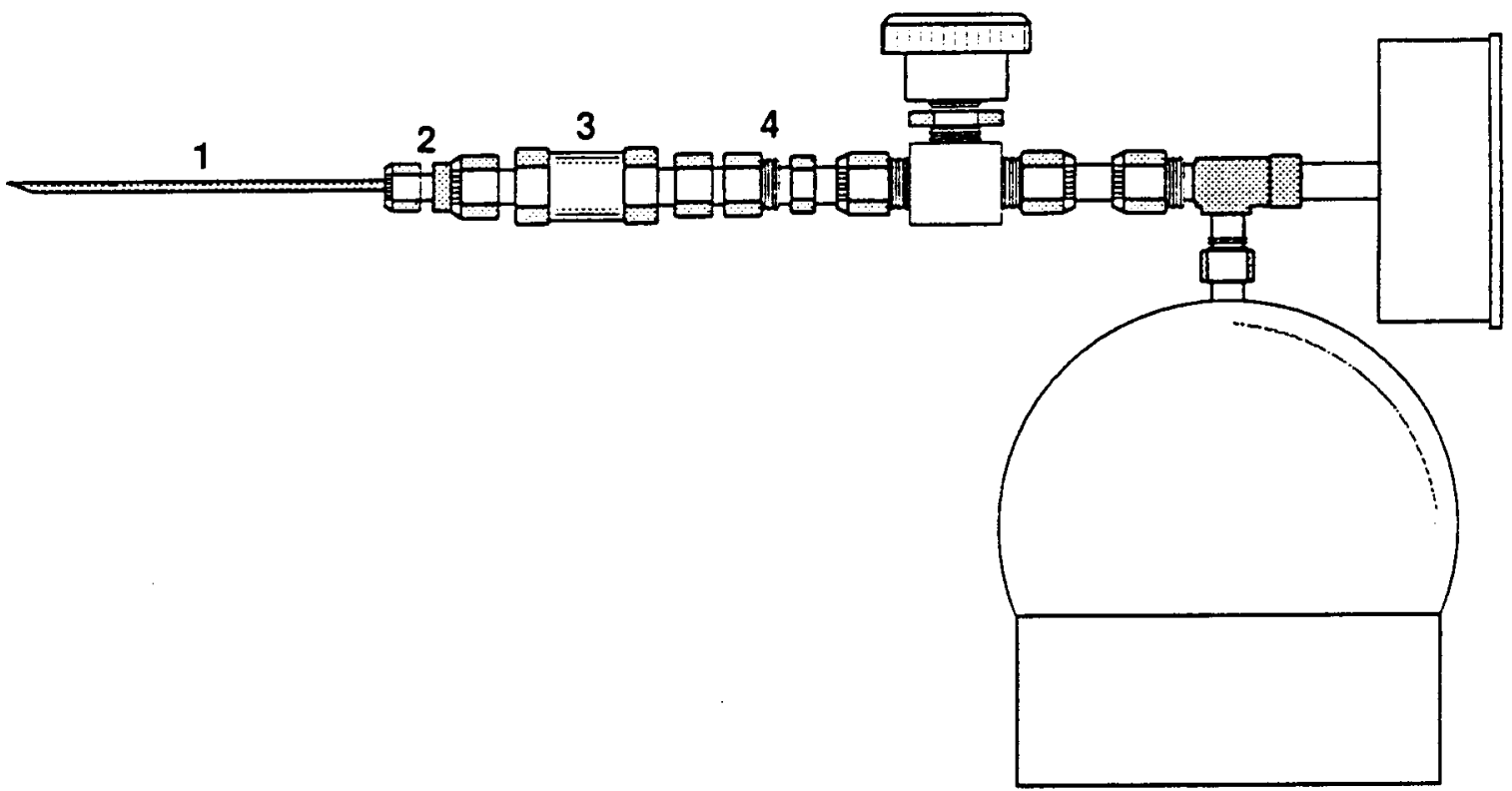

Figure 2. Direct Canister Headspace Sampling Apparatus

$110.2-4$ 
collected. A dial pressure/vacuum gauge is mounted on each sample canister used for direct canister sampling. The direct canister sampling apparatus components are listed in Table 1.

2.4 Sample Canister: A sample canister is a leak-free welded stainless steel SUMMA ${ }^{\oplus}$ pressure vessel with a $\mathrm{Cr}$-NiO-passivated interior surface, bellows valve, and dial pressure/vacuum gauge. All sample canisters have $V_{C R}{ }^{\oplus}$ fittings for connection to sampling and analytical equipment. When using a low internal volume sampling manifold, the dial pressure/vacuum gauge is not required if an organic vapor analyzer with a photoionization detector (PID) is used to check for sampling manifold cleanliness prior to sampling. A dial pressure/vacuum gauge on a sample canister indicates if the canister has leaked, thereby preventing sampling manifold contamination by exposure to a potentially contaminated sample canister. In the absence of this canister gauge, a PID may be used along with confirmatory sampling manifold blanks to check for manifold cleanliness.

SUMMA ${ }^{\oplus}$ canisters have been used in the past for VOC sample collection and have a demonstrated sample storage stability for many specific VOCs (References 1 and 3). Two sizes of canisters (150 $\mathrm{mL}$ and $250 \mathrm{~mL}$ ) are used for the collection of headspace gas samples.

2.5 Pressure and Temperature Measurement Apparatus: When using the direct canister method, sample canister pressure and ambient pressure are monitored and recorded during sampling operations. When using the low internal volume sampling manifold, the sampling manifold pressure, read with the capacitance manometer, is monitored and recorded. In both cases, ambient temperature is also monitored and recorded.

2.5.1 Pressure Gauges: A Fortin barometer is used to monitor ambient pressure, while a capacitance manometer is used to monitor both sampling manifold pressure and sample canister pressure. A dial pressure/vacuum gauge is used to monitor sample canister pressure when a sampling manifold equipped with a capacitance manometer is not available.

2.5.1.1 Ambient Pressure Sensor: The ambient pressure sensor is a full-range Fortin barometer, or equivalent, with a range of approximately 500 to $800 \mathrm{~mm} \mathrm{Hg}$. It is kept in the sampling area. Its resolution is $1 \mathrm{~mm} \mathrm{Hg}$ and its calibration is traceable to National Institute of Standards and Technology (NIST) standards. A dual scale thermometer should be included on the barometer case for temperature corrections. 
Revision: 0

Date: August 30, 1991

Table 1. Direct Canister Sampling Apparatus:

List of Major Components

\begin{tabular}{|c|c|c|}
\hline Component & Component Description & Purpose \\
\hline 1 & $1 / 8 "$ SS tubing $w /$ sharpened tip & $\begin{array}{l}\text { Pierce innermost layer of } \\
\text { confinement }\end{array}$ \\
\hline 2 & $1 / 4^{\prime \prime}$ to $1 / 8^{\prime \prime}$ reducer & Adapt needle to filter \\
\hline 3 & $\begin{array}{l}\text { Compact in-line filter }(0.5 \mu \mathrm{m} \\
\text { sintered stainless steel) }\end{array}$ & $\begin{array}{l}\text { Prevents particulate } \\
\text { contamination from entering } \\
\text { sampling apparatus }\end{array}$ \\
\hline 4 & $1 / 4$ "Swagelok $^{\oplus}$ to $\mathrm{VCR}^{\oplus}$ adapter & $\begin{array}{l}\text { Allows repetitive leak tight } \\
\text { connection using disposable } \\
\text { metal gasket }\end{array}$ \\
\hline 5 & $\begin{array}{l}\text { SUMMA }{ }^{\oplus} \text { canister with bellows } \\
\text { valve and dial vacuum/pressure } \\
\text { gauge }\end{array}$ & Sample container \\
\hline
\end{tabular}


2.5.1.2 Dial Pressure/Vacuum Gauge: This type of gauge is helium leak tested to $1.5 \times 10^{-7}$ standard $\mathrm{cc} / \mathrm{sec}$, has all stainless steel construction, and is capable of tolerating temperatures to $125^{\circ} \mathrm{C}$. The gauge is able to indicate $100 \mathrm{psig}$ pressure to a vacuum of 30 inches of mercury.

2.5.1.3 Capacitance Manometer: The capacitance manometer is pneumatically connected to the sampling manifold. The pressure sensor consists of a pressure transducer and electronics/display unit. The transducer is able to measure absolute pressure in the range from 0.05 $\mathrm{mm} \mathrm{Hg}$ to $100 \mathrm{~mm} \mathrm{Hg}$ with a usable resolution of $\pm 0.005 \mathrm{~mm} \mathrm{Hg}$ at $0.05 \mathrm{~mm} \mathrm{Hg}$.

2.5.2 Thermometer: The thermometer is a NIST-traceable laboratory grade mercury thermometer, total immersion type, with nitrogen above the mercury. It has permanent stain markings in the approximate range of $-30^{\circ}$ to $50^{\circ} \mathrm{C}$, with a resolution of $1^{\circ} \mathrm{C}$.

2.6 Organic Vapor Analyzer With a Photoionization Detector (PID): The PID is a battery powered portable organic vapor analyzer capable of measuring volatile organic compounds in air in two ranges. In the range of $0.200 \mathrm{ppm}$, it has a resolution of $0.1 \mathrm{ppm}$. In the range of $200-2000 \mathrm{ppm}$, it has a resolution of $1.0 \mathrm{ppm}$. The PID uses a $11.8 \mathrm{eV}$ lamp allowing it to detect most of the target analytes listed in Table 3-5 of the QAPP. Cylinders of both zero air and $250 \mathrm{ppm}$ isobutylene are supplied by the manufacturer for calibrating the unit.

\subsection{REAGENTS AND MATERIALS}

Not applicable.

\subsection{SAMPLE HANDLING AND PROCEDURES}

4.1 Procedures for Cleaning the Low Internal Volume Sampling Manifold: This section describes procedures for cleaning the low internal volume sampling manifold. These procedures are provided to ensure the sample collected using this apparatus is representative by reducing the possibility of contamination by manufacturing impurities and ambient air. Use of these cleaning procedures will also reduce the possibility of cross-contamination between sampling operations. These procedures are as follows:

- Section 4.1.1 is a procedure for initially cleaning and leak-checking the sampling manifold.

- Section 4.1.2 is a procedure for routine cleaning and leak-checking the sampling manifold. 
Revision: 0

Date: August 30, 1991

- Section 4.1.3 is a procedure for cleaning the sampling manifold after collection of a field reference standard.

- Section 4.1.4 is a procedure that describes the method for needle assembly cleaning.

- Section 4.1.5 is a procedure that describes sample canister cleaning.

4.1.1 Initial Sampling Manifold Cleaning and Leak-Free Certification: Follow the method specified in Procedure 110.1, Section 4.2.1.

4.1.2 Routine Sampling Manifold Cleaning and Leak Check: The following procedure assumes that a clean needle assembly (1-3) has been connected to valve (4) and inserted into purge assembly tee (37). The procedure also assumes that all sampling manifold valves are closed, the vacuum pump is operating, and all regulators for the high pressure cylinders (zero air, helium or nitrogen, and field reference standards, etc.) have been properly purged and set to a delivery pressure of 10 psi. Refer to Figure 1 for location of numbered components.

1. Select the sample canister(s) to be used for sampling and if a dial pressure/vacuum gauge is present, check the reading (lightly tap on the gauge face plate to verify reading). Record the reading on the sample canister tag. Attach the sample canister(s) to the sampling manifold and tighten connector(s). Sample canister port(s) not occupied by a canister must be closed with a cap or valve. If the port to which a canister is attached has an optional valve (not shown) attached to the manifold port tee, open this valve prior to proceeding to step 2 . Leave the sample canister valve closed.

2. Open valves (39), (23), (21), and (33).

3. Slowly open valve (35) until rotameter $(40)$ indicates a flow rate of approximately 2 liters per minute.

4. Open valves (16a) and $(16 \mathrm{~b})$ and slowly open valve (4) until rotameter $(40)$ indicates a flow rate of approximately 1 liter per minute. The sampling manifold is now being purged with zero air. Purge for approximately 3 minutes, periodically checking and adjusting valves (35) and (4), if necessary, to maintain rotameter flow rate at 1 liter per minute.

5. Close valve (4) to evacuate the sampling manifold. Wait 5 seconds, close valve (16a), and reopen valve (4) to allow the sampling manifold to fill with zero air. Repeat this 3 to 4 times. Terminate this procedure by closing valve (16b), opening valve (4), and allowing the sampling manifold to fill with zero air. If a PID is attached to a valve at canister port (15), open valve (15) and turn on the PID. Observe the PID display for at least 10 seconds. If the PID indicates a VOC concentration of $0.5 \mathrm{ppm}$ or greater, continue purging and sweeping the sampling manifold with zero air until the PID indicates less than 0.5 $\mathrm{ppm}$. When the PID indicates less than $0.5 \mathrm{ppm}$, the internal volume between valve (7) and transducer $(8)$ is purged by opening valve (7).

CAUTION: Care should be taken to slowly evacuate and fill the sampling manifold, since rapid fluctuations in manifold pressure may damage the manometer when valve (7) is open. 
Revision: 0

Date: August 30, 1991

6. Open valve (7) and close valve (4).

7. Slowly open valve (16b) and allow the vacuum pump to reduce the pressure in the sampling manifold to $0.05 \mathrm{~mm} \mathrm{Hg}$, as indicated by the manometer (9).

8. Once the sampling manifold pressure has been reduced to $0.05 \mathrm{~mm} \mathrm{Hg}$, close valve (16a).

9. Observe the manometer (9) reading for 3 to 5 seconds to verify that all sampling manifold valves have been properly closed and no observable leaks are present. If the manometer indicates a leak, cease sampling activities and leak check the manifold as described in steps 3 through 5 of Procedure 4.3.1.

10. Glancing up at the manometer (9) to check for changes in pressure, open sample canister valve(s). Record the pressure reading on the sample canister tag.

NOTE: An increase in manifold pressure greater than $0.02 \mathrm{~mm} \mathrm{Hg}$ indicates a canister leak, and opening the remaining sample canister valves is not advised. All open valves are closed and the exposed sample canisters are removed and replaced. Then the manifold is purged again following steps 4 through 9, above.

11. If no leaks are detected, the sampling manifold is ready to collect a headspace gas sample(s) and OC samples, as appropriate.

\subsubsection{Sampling Manifold Cleaning After Field Reference Standard Collection: This}

procedure is performed immediately after collecting a field reference standard in accordance with Section 4.2.5. It assumes that the needle assembly (1-3) used for the field reference standard collection is still inserted into the purge assembly tee (37). Refer to Figure 1 for location of numbered components.

1. Close valve (33).

2. Close valve (39) and open valves (4) and (23).

3. Observe the manometer (9) reading until the pressure ceases to increase and then close valve (23).

CAUTION: Care should be taken to slowly evacuate and fill the sampling manifold, since rapid fluctuations in manifold pressure may damage the manometer when valve (7) is open.

4. Open valve (16a), slowly open valve (16b), and evacuate the sampling manifold for a minimum of 5 seconds.

5. Close Valve (16b) and then slowly open valve (23).

6. Repeat steps 2 through 4 three more times. Terminate this step by closing valve (16b).

7. Close valve (4), open valve (39), and adjust valve (35) until rotameter $(40)$ indicates approximately 1 liter per minute. 
8. Close valve (23).

9. Disconnect the needle assembly from the purge assembly tee (37) and connect a clean needle assembly to valve (4).

10. Insert needle assembly ( $1-3$ ) into purge assembly tee (37) and proceed with the cleaning described in Section 4.1.2.

4.1.4 Needle Assembly Cleaning: Follow the method specified in Procedure 110.1, Section 4.2.4, if needle assembly is not disposed of.

4.1.5 Sample Canister Cleaning: Follow the method specified in Guidance Manual Procedure 210.1.

\subsection{Procedures for Collecting Headspace Gas and Quality Control Samples using a Low Internal} Volume Sampling Manifold: Headspace gas and quality control samples collected using the sampling manifold and procedures described here comply with the headspace gas sampling data quality and quality assurance objectives (see QAPP Sections 1.4 and 3.3.2). These procedures are as follows:

- Section 4.2.1 is a procedure for collecting headspace gas samples.

- Section 4.2.2 is a procedure for collecting field duplicates.

- Section 4.2 .3 is a procedure for collecting field blanks.

- Section 4.2.4 is a procedure for collecting sampling manifold blanks.

- Section 4.2.5 is a procedure for collecting field reference standards.

NOTE: Prior to sampling, drums are allowed to equilibrate to the temperature of the sampling area for at least $\mathbf{7 2}$ hours.

CAUTION: Appropriate site radiological controls must be established prior to removing the drum lid.

4.2.1 Headspace Gas Sample Collection: The following procedure describes the use of the low internal volume sampling manifold for collection of headspace gas samples from within innermost layers of confinement. Refer to Figure 1 for location of numbered components of the sampling manifold. Headspace gas samples are collected from within all innermost layers of confinement that meet the criteria specified in Section 1.2. Sample canisters are evacuated to 0.05 $\mathrm{mm} \mathrm{Hg}$ prior to sample collection. 
1. Verify that the sampling manifold has been properly cleaned and leak-checked as described in Section 4.1.2.

2. Close valve (7).

3. Prepare the drum for sampling by removing the drum lid and the internal rigid liner lid which should expose the 55-gallon poly liner bag.

4. After sampling the headspace gas of the $\mathbf{5 5}$ gallon poly liner bag, locate an area within an innermost layer of confinement from which a representative headspace gas sample can be withdrawn. Care should be taken to avoid placing the tip of the needle in an area where solid material or particulate matter might fill or clog the needle.

5. Remove the needle assembly (1-3) from the purge assembly tee (37) and press the needle through the poly bag and into the headspace gas. Disturbing the bag should be avoided, if possible. However, if handling the bag is necessary, the operator should do so in a manner that minimizes the potential for introduction of outside air into the poly bag's headspace gas.

6. Hold the needle in the headspace and open valve (4). Hold the needle steady until the canister gauge indicates zero psig.

7. Close the sample canister(s) valve(s).

8. Remove the needle (1) from the poly bag and disconnect the needle assembly from valve (4). Place the needle assembly in a collection box for items to be cleaned or dispose of it.

9. Remove a fresh needle assembly from its resealable bag and connect the needle assembly to valve (4). Insert the fresh needle assembly into the purge assembly tee (37) and hand tighten the assembly in place.

10. Remove the sample canister(s) from the sampling manifold and log the date, time, sampling manifold pressure (9), ambient pressure, and ambient temperature on the sample canister tag (see QAPP Section 5, Sample Canister Tag).

11. Note on the $Q C$ sample record the type of sample that the sample canister(s) contains (see OAPP Section 5, OC Samp/e Record Form) and place the sample canister in the shipping container.

12. Clean the sampling manifold according to the procedure in Section 4.1.2.

4.2.2 Field Duplicate Collection: The following procedure describes the use of the low internal volume sampling manifold for collection of field duplicate samples. These samples are used to assess the precision of the sampling and analytical process. Field duplicates are collected at a frequency of one per bin (i.e., every six drums) or one per twenty innermost layers of confinement sampled, whichever is more frequent. The procedure for field duplicate collection is similar to that specified in Section 4.2.1 for headspace gas sample collection. However, when collecting field duplicates, two sample canisters are attached to the sampling manifold. One canister is attached at 
port (10) and the other is attached at port (11). All other sample canister ports are isolated by valve (16a), which is closed. When collecting field duplicates, valves (10) and (11) are closed prior to opening valve (4). After opening valve (4), valve $(10)$ is opened and the first sample canister filled. Then valve (11) is opened and the second sample canister is filled. Finally, valve (4) is closed followed by valves $(10)$ and (11).

4.2.3 Field Blank Collection: Field blanks are used to assess background levels of Program specified analytes. Field blanks may be collected with or without the use of the sampling manifold. Sampling sites that choose to collect field blanks through the sampling manifold collect a sampling manifold blank (Section 4.2.4) immediately prior to collecting the field blank. Also prior to field blank collection, sampling sites should use a PID to obtain a real time indication of sampling manifold cleanliness. Field blanks are collected at a frequency of one per day.

\subsubsection{Field Blank Collection Without the Sampling Manifold: The following} procedure can be used for the collection of a field blank directly into a sample canister.

1. After the lid of the waste drum has been removed and the $\mathbf{5 5}$ gallon poly liner bag has been opened, place a sample canister near the top of the drum and open the sample canister valve.

2. Allow the sample canister to equilibrate to ambient pressure. Verify that the dial vacuum/pressure gauge indicates zero psig. Close the canister valve.

3. Log the date, time, sample canister pressure, ambient pressure, and ambient temperature on the canister tag (see QAPP Section 5, Sample Canister Tag).

4. Note on the $\mathrm{OC}$ sample record that the sample canister contains a field blank (see QAPP Section 5, QC Sample Record Form) and place the sample canister in the shipping container.

4.2.3.2 Field Blank Collection Via the Sampling Manifold: The following procedure can be used for collection of a field blank using the sampling manifold.

1. Verify that the sampling manifold has been properly cleaned and leak-checked as described in Section 4.1.2.

2. Prepare a drum by removing the drum lid and the internal rigid liner lid. Open the 55-gallon poly liner bag.

3. Remove the needle assembly (1-3) from the purge assembly tee (37) and hold the needle assembly near the top of the drum.

4. Open valve (4). Hold the needle steady until the manometer (9) indicates ambient pressure, or ceases to show an increase in pressure. Verify that the canister gauge, if present, indicates zero psig. 
5. Close the sample canister(s) valve(s).

6. Disconnect the needle assembly from valve (4). Place the needle assembly in a collection box for items to be cleaned or dispose of it.

7. Remove a clean needle assembly from its resealable bag and connect the needle assembly to valve (4). Insert the clean needle assembly into the purge assembly tee (37) and hand tighten the assembly in place.

8. Remove the sample canister(s) from the sampling manifold and log the date, time, sampling manifold pressure (9), ambient pressure, and ambient temperature on the sample canister tag (see QAPP Section 5, Sample Canister Tag).

9. Note on the QC sample record that the sample canister(s) contains a field blank (see QAPP Section 5, OC Sample Record Form) and place the sample canister in the shipping container.

10. Clean the sampling manifold according to the procedure in Section 4.1.2.

4.2.4 Sampling Manifold Blank Collection: The following procedure describes the use of the low internal volume sampling manifold for collection of sampling manifold blanks. These samples are used to assess the cleanliness of the sampling manifold. A sampling manifold blank is collected and analyzed prior to first use of the sampling manifold, then at a frequency of one per day prior to sample collection. Refer to Figure 1 for location of numbered components.

1. Verify that the sampling manifold has been properly cleaned and leak-checked as described in Section 4.1.2.

2. With the needle assembly (1-3) connected to the purge assembly tee (37), adjust valve (35) until rotameter $(40)$ indicates a flow rate of approximately 10 liters per minute of zero air.

3. Open valve (4) to allow the sample canister to fill as quickly as possible without reducing the flow indicated by the rotameter $(40)$ to zero.

NOTE: If rotameter $(40)$ indicates zero flow, the sampling manifold blank is invalidated and the sampling manifold is cleaned (Section 4.1.2) prior to collection of another sampling manifold blank.

4. Continue drawing zero air from the purge assembly tee (37) until the manometer (9) indicates ambient pressure or ceases to show an increase in pressure. Verify that the sample canister gauge, if present, indicates zero psig.

5. Close valve (4) and the sample canister valve when the manometer indicates ambient pressure.

6. Adjust valve (35) to reduce the flow rate through the rotameter (40) to less than 1 liter per minute (to conserve zero air).

7. Remove the sample canister from the manifold. 
Revision: 0

Date: August 30, 1991

8. Log the date, time, manifold pressure (9), ambient pressure, and ambient temperature on the sample canister tag (see QAPP Section 5, Sample Canister Tag).

9. Note on the OC sample record that the sample canister contains a sampling manifold blank (see QAPP Section 5, QC Sample Record Form) and place the canister in the shipping container.

4.2.5 Field Reference Standard Collection: The following procedure describes the use of the low internal volume sampling manifold for collection of field reference standards. These samples are used to assess the accuracy of the sampling and analysis process. A field reference standard is collected and analyzed prior to first use of the sampling manifold, then at a frequency of one per bin for the first two bins, followed by quarterly collection, thereafter. Refer to Figure 1 for location of numbered components.

1. Verify that the sampling manifold has been properly cleaned and leak-checked as described in Section 4.1 .2 and verify that canister port (11) is closed.

2. Close valve (23), open valve (32), and open the valve (28) through (31) to the cylinder of reference standard gas from which a field reference standard sample is desired. Allow reference standard gas to flow through the purge assembly for 2-3 minutes, opening and closing valve $(35)$ a few times to facilitate purging residual zero air from the pneumatic lines.

3. With the needle assembly (1-3) connected to the purge assembly tee (37), adjust valve (35) until rotameter $(40)$ indicates a flow rate of approximately 10 liters per minute.

4. Open valve (4) to allow the sample canister (10) to fill as quickly as possible without reducing the flow indicated by the rotameter $(40)$ to zero.

NOTE: If rotameter (40) indicates zero flow, the field reference standard is invalidated and the sampling manifold is cleaned (Section 4.1.3) prior to collection of another field reference standards sample.

5. Continue drawing field reference standard gas from the purge assembly tee (37) until the manometer (9) indicates ambient pressure or ceases to show an increase in pressure. Verify that the sample canister gauge, if present, indicates 0 psig.

6. Close valve (4) and the sample canister valve when the manometer indicates ambient pressure.

7. Adjust valve (35) to reduce the flow rate through rotameter $(40)$ to approximately 1 liter per minute, close valve (32), and close the selected open reference standard gas valve (28$31)$.

8. Open valve (23) and open and close valve $(35)$ a few times to facilitate purging residual field reference standard gas from the pneumatic lines. Terminate this step with valve (35) adjusted to deliver approximately 1 liter per minute zero air through rotameter (40).

9. Remove the sample canister from the sampling manifold. 
10. Log the date, time, manifold pressure (9), ambient pressure, and ambient temperature on the sample canister tag (see QAPP Section 5, Sample Canister Tag).

11. Note on the $Q C$ sample record that the sample canister contain a field reference standard (see QAPP Section 5, OC Sample Record Form) and place the sample canister in the shipping container.

12. Close the sample canister port and proceed with cleaning the sampling manifold as described in Section 4.1.3.

4.3 Procedures for Cleaning the Direct Canister Sampling Apparatus: Procedures for cleaning the sampling apparatus are provided to ensure the sample is representative by reducing the possibility of contamination from manufacturing impurities or ambient air. Use of these cleaning procedures will also reduce the possibility of cross-contamination between sampling operations. These procedures are as follows:

- Section 4.3.1 is a procedure for cleaning the individual components of the sampling apparatus prior to assembly.

- Section 4.3.2 is a procedure for cleaning the needle and filter between samples. NOTE: Needles and filters may be disposed of after each sampling operation.

\subsubsection{Initial Sampling Apparatus Cleaning and Leak-Check: Prior to assembly,} components of the sampling apparatus are inspected and thoroughly cleaned using the following procedure.

NOTE: Parts received free of contamination, as stated or certified by the manufacturer, may be exempted from this cleaning procedure at the discretion of sampling personnel.

1. Place stainless steel parts and fittings in a beaker of reagent grade hexane in an ultrasonic bath for 15 minutes. Repeat this procedure with reagent grade methanol. Stainless steel parts and fittings may also be cleaned by assembling and forcing steam through them. Rinse the stainless steel parts with HPLC grade water and dry parts in an oven at $100^{\circ} \mathrm{C}$ for at least 12 hours.

2. Assemble the sampling apparatus and leak-check by pressurizing to 10 psig and placing soap solution on all fittings and connections. For a period of 30 minutes, periodically check for the production of bubbles. The sampling apparatus may also be pressurized with helium and leak-checked with a helium detection device.

3. Tighten fittings and connections that leak until no leaks are observed. From clean spare parts, replace any fittings and connections that continue to leak.

4. Purge the sampling apparatus with humid zero air and cap open connections for storage. 
4.3.2 Needle and Filter Cleaning: This procedure describes the steps for cleaning the needle and filter after headspace gas samples or field reference standards have been collected. With slight modifications, this procedure can also be used to batch clean the needle and filters, prior to use. In lieu of cleaning, the needle and filter may be disposed of after headspace gas sample or field reference standard collection. Refer to Figure 2 for location of numbered components of the direct canister sampling apparatus.

1. After headspace gas samples or field reference standards have been collected, detach the needle (1) and filter (3) at the VCR ${ }^{\oplus}$ fitting (4) and dispose of the VCR ${ }^{\oplus}$ gasket.

CAUTION: Needle and filter may be radiologically contaminated.

2. Remove the filter assembly to an area where it can safely be disassembled and cleaned.

3. Unscrew the housing of the filter and firmly grasp the filter element with a sturdy pair of forceps. Firmly pull the element to dislodge it from the housing. Properly discard the element.

4. Place the reusable parts in a beaker of reagent grade hexane in an ultrasonic bath for 15 minutes. Repeat this procedure with reagent grade methanol. The needle assembly may also be cleaned by screwing the filter housing together again and forcing steam through the entire assembly. Rinse the parts with HPLC grade water and dry in an oven at $100^{\circ} \mathrm{C}$ for at least 12 hours.

5. Insert a new filter element into the filter and tightly screw the body together.

6. Purge the needle and filter with zero air as the needle and filter are being placed into a zero air filled resealable bag.

7. Press a Teflon ${ }^{\oplus}$ plug over the tip of the needle, close the resealable bag and place it in a box for storage.

\subsection{Procedures for Collecting Headspace Gas and Quality Control Samples using the Direct}

Canister Sampling Apparatus: Headspace gas and quality control samples collected using the following procedures comply with the headspace gas sampling data quality and quality assurance objectives included in the QAPP (see Sections 1.4 and 3.3.2). These procedures are as follows:

- Section 4.4.1 is a procedure for collecting headspace gas samples.

- Section 4.4.2 is a procedure for collecting field duplicates.

- Section 4.4 .3 is a procedure for collecting field blanks.

- Section 4.4.4 is a procedure for collecting equipment blanks.

- Section 4.4 .5 is a procedure for collecting field reference standards. 
Revision: 0

Date: August 30, 1991

NOTE: Prior to sampling, drums are allowed to equilibrate to the temperature of the sampling area for at least $\mathbf{7 2}$ hours.

CAUTION: Appropriate site radiological controls must be established prior to removing the drum lid.

\subsubsection{Direct Canister Innermost Laver of Confinement Headspace Gas Sample Collection:}

The innermost layer of confinement sampling method described below employs the direct canister sampling apparatus (see Figure 2) to collect headspace gas samples from within innermost layers of confinement which meet the minimum criteria specified in Section 1.2. Sample canisters are evacuated to $0.05 \mathrm{~mm} \mathrm{Hg}$ prior to sample collection.

1. Verify that sample canister is evacuated by observing the sample canister dial vacuum/pressure gauge indicates a vacuum of 30 inches of mercury.

2. Locate an area within the inner bag from which a headspace gas sample can be withdrawn. Care should be taken to avoid placing the tip of the needle in an area where solid material or particulate matter might fill or clog the needle.

3. Remove the cap from the tip of the needle and press the needle through the innermost layer of confinement and into the headspace gas. Disturbing the bag should be avoided, if possible. If pulling on the bag is necessary, do so in a manner that will minimize the potential for introducing outside air into the poly bag's internal gas space.

4. While holding the needle in the inner bag's headspace, open the sample canister valve to allow headspace gas to expand into the canister.

5. Observe the vacuum gauge on the sample canister while the sample is being collected. When the gauge indicates zero psig, close the sample canister valve and withdraw the needle from the bag.

6. Log the date, time, sample canister pressure, ambient pressure, and ambient temperature on the sample canister tag (see OAPP Section 5, Sample Canister Tag).

7. Note on the $O C$ sample record that the sample canister contains a headspace gas sample (see QAPP Section 5, OC Sample Record Form) and place the sample canister in a shipping container.

4.4.2 Direct Canister Field Duplicate Collection: The following procedure describes the use of the direct canister sampling apparatus (see Figure 2) for collection of field duplicate samples. These samples are used to assess the precision of the sampling and analytical process. Field duplicates are collected at a frequency of one per bin (i.e., six drums) or one per twenty innermost layers of confinement sampled, whichever is more frequent. The procedure for field duplicate collection is similar to that specified in Section 4.4.1 for headspace gas sample collection. However, when 
collecting field duplicates, two sample canisters are filled sequentially with headspace gas from within the same innermost layer of confinement.

4.4.3 Direct Canister Field Blank Collection: The following procedure describes the use of the direct canister sampling apparatus (see Figure 2) for collection of field blanks. Field blanks are used to assess background levels of Program required analytes. Field blanks are collected at a frequency of one per day.

1. After the 55-gallon poly bag has been opened, place a sample canister near the inner poly bags, remove the cap from the tip of the needle, and open the sample canister valve.

2. Allow the sample canister to equilibrate to ambient pressure, then close the canister valve.

3. Log the date, time, sample canister pressure, ambient pressure, and ambient temperature on the canister tag (see QAPP Section 5, Sample Canister Tag).

4. Note on the QC sample record that the sample canister contains a field blank (see QAPP Section 5, OC Sample Record Form) and place the sample canister in the shipping container.

4.4.4 Direct Canister Sampling Equipment Blank Collection: The following procedure describes the use of the direct canister sampling apparatus for collection of sampling equipment blanks. These samples are used to assess the cleanliness of the sampling apparatus. An equipment blank is collected and analyzed prior to first use of the direct canister sampling apparatus, then at a frequency of one per day. Sampling equipment blanks can be collected by two methods; the canister method described in Section 4.4.4.1. or the purge assembly method described in Section 4.4.4.2.

4.4.4.1 Sampling Manifold Method: If a sampling manifold exists for taking headspace gas samples from the 55-gallon poly bag and the needle and filter used on the manifold are cleaned in the same batch and are identical to those used on the direct canister sampling apparatus, then the sampling manifold blank can be used to verify that the needle and filter used on the direct canister sampling apparatus is clean.

4.4.4.2 Purge Assembly Method: Where a sampling manifold does not exist a purge assembly similar to that used with the sampling manifold can be constructed in the laboratory and used to collect direct canister sampling equipment blanks. The essential components of a laboratory purge assembly are illustrated in Figure 3.

The compressed gas cylinders supply zero air for collection and the humidifier conditions this gas prior to collection. The rotameter indicates gas flow rate and is monitored to assure ambient air is not 


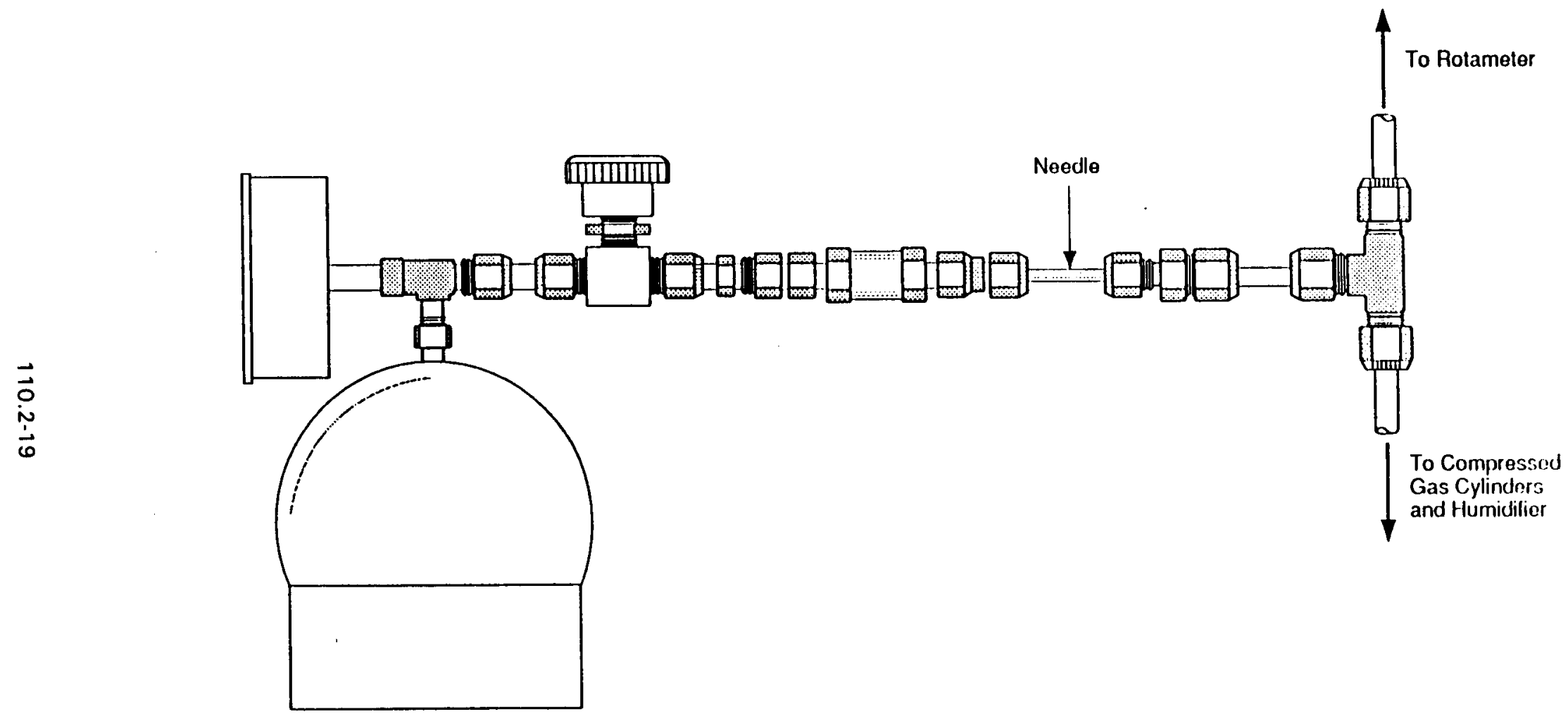

Figure 3. Purge Assembly Method Schematic 
drawn into the sample canister during equipment blank collection. The tee in the purge line is adapted for leak tight connection to the $1 / 8^{n}$ needle of the direct canister sampling apparatus.

The following procedure assumes that all valves are closed and that the regulator on the zero air cylinder has been purged and set to a delivery pressure of 10 psi.

1. Select a sample canister with attached needle and filter to be used for equipment blank collection (lightly tap on the gauge face plate to verify reading). Enter the reading on the sample canister tag.

2. Insert the sampling apparatus needle into the purge assembly and tighten the fitting.

3. Adjust the valve on the zero air cylinder until the rotameter indicates a flow rate of approximately 1 liter per minute.

4. Purge the line for several minutes.

5. Adjust the valve on the zero air cylinder until the rotameter indicates a flow rate of approximately 10 liters per minute.

6. Open the sample canister valve to allow the sample canister to fill as quickly as possible without reducing the flow indicated by the rotameter to zero.

NOTE: If the rotameter indicates zero flow during this process, then the sample is invalidated.

7. Continue drawing zero air from the purge assembly until the sample canister pressure gauge indicates zero psig.

8. Close the sample canister valve.

9. Close the valve on the zero air cylinder.

10. Disconnect the sampling apparatus needle from the purge tee.

11. Log on the sample canister tag the date, time, sample canister pressure, ambient pressure, and ambient temperature (see QAPP Section 5, Sample Canister Tag).

12. Note on the $Q C$ sample record that the sample canister contains a sampling equipment blank (see QAPP Section 5, OC Sample Record Form) and place that canister in a shipping container.

\subsubsection{Direct Canister Field Reference Standard Collection: The following procedure} describes the use of the direct canister sampling apparatus for collection of field reference standards. These samples are used to assess the accuracy of the sampling and analysis process. A field reference standard is collected and analyzed prior to first use of the sampling apparatus, then at a frequency of one per bin for the first two bins, followed by quarterly collection, thereafter. Field reference standards 
can be collected by either of two methods; the canister method described in Section 4.4.5.1. or the purge assembly method described in Section 4.4.5.2.

4.4.5.1 Sampling Manifold Method: If a sampling manifold exists for taking headspace gas samples from the 55-gallon poly bag and the needle and filter used on the manifold are cleaned in the same batch and identical to those used on the direct canister sampling apparatus, then the field reference standard collected on the sampling manifold can be used to verify that the needle and filter used on the direct canister sampling apparatus do not affect analyte recovery.

4.4.5.2 Purge Assembly Method: Where a sampling manifold does not exist a purge assembly similar to that used with the sampling manifold can be constructed in the laboratory and used to collect direct canister field reference standards. The essential components of a laboratory purge assembly are illustrated in Figure 3.

The following procedure assumes that all valves are closed and that the regulator on the reference standard gas cylinder has been purged and set to a delivery pressure of $10 \mathrm{psi}$.

1. Select a sample canister with attached needle and filter to be used for field reference standard collection (lightly tap on the gauge face plate to verify reading). Enter the reading on the sample canister tag.

2. Insert the sampling apparatus needle into the purge assembly tee and tighten the fitting.

3. Adjust the valve on the reference standard gas cylinder until the rotameter indicates a flow rate of approximately 1 liter per minute.

4. Purge the line for several minutes.

5. Adjust the valve on the reference standard gas cylinder until the rotameter indicates a flow rate of approximately 10 liters per minute.

6. Open the sample canister valve to allow the sample canister to fill as quickly as possible without reducing the flow indicated by the rotameter to zero.

NOTE: If the rotameter indicates zero flow during this process, then the sample is invalidated.

7. Continue drawing reference standard gas from the purge assembly until the sample canister pressure gauge indicates zero psig.

8. Close the sample canister valve.

9. Close the valve on the reference gas cylinder.

10. Disconnect the sampling apparatus needle from the purge tee. 
11. Log on the sample canister tag the date, time, sample canister pressure, ambient pressure, and ambient temperature (see QAPP Section 5, Sample Canister Tag).

12. Note on the $Q C$ sample record that the sample canister contains a field reference standard (see OAPP Section 5, OC Sample Record Form) and place that canister in a shipping container.

4.5 Radiological Considerations: The use of the direct canister sampling apparatus requires the transfer of sample canisters into and out of a sampling area which may be contaminated by radionuclides. The following steps are general guidelines for this transfer.

NOTE: Each sampling site must obtain approval from appropriate operations and/or transportation personnel for proper radiological controls.

1. Prepare sample canisters to be sent into a contaminated area by taping all external surfaces, except for the valve handle, with duct tape.

2. Place each sample canister in a plastic bag and tape to seal.

3. After sample collection, wipe the plastic covering of the sample canister and obtain a radiation level reading.

4. Properly dispose of contaminated plastic and tape.

\subsection{CALCULATIONS}

5.1 See Section 5.0 of Procedure 110.1 for a discussion of calculations to compute the expected sample canister gauge pressure change resulting from ambient temperature and pressure changes.

\subsection{ANALYTICAL PROCEDURE}

6.1 The procedures for the determination of VOCs and gases in the headspace of waste containers are provided as Procedures 430.1, 430.2, and 440.1 for VOCs, and 510.1, 520.1, and 530.1 for gases.

\subsection{QUALITY CONTROL FOR HEADSPACE GAS SAMPLES}

7.1 To prevent cross contamination, the needle and filter of the direct canister apparatus are disposed of or, if reused, cleaned between sample collections according to the procedure in Section 4.1.4. As a further $\mathrm{QC}$ measure, the needle and filter, after cleaning, are purged with zero air, 
nitrogen, or helium and capped for storage to prevent sample contamination by VOCs present in the ambient air. Field duplicates are collected to assess sampling and analytical precision. Field blanks and field reference standards are also collected.

7.2 Specific sampling steps to ensure that the headspace gas samples are representative include:

- Sample canister cleaning and leak checking

- Use of sample canisters with a passivated internal surface

- Use low internal volume sampling apparatus

- Small sample volume: low sample volume to available headspace gas volume ratio

- Careful pressure regulation in sample canister

- Performance audits

- Collection of field blanks, field reference standards, equipment blanks, and field duplicates.

7.3 Quality Control for Pressure and Temperature Measurements: This section pertains to the calibration and certification of equipment and instrumentation used for temperature and pressure measurements. All information related to the calibrations of the pressure and temperatures sensors is recorded.

7.3.1 Certification Check of the Ambient Pressure Sensor: The ambient pressure sensor is certified by the manufacturer using standards or equipment traceable to the NIST. The pressure indication of the sensor is corrected in accordance with temperature indicated by a dual-scale thermometer attached to its casing, if necessary.

7.3.2 Calibration of the Capacitance Manometer: The capacitance manometer is certified using standards or equipment traceable to the NIST.

7.3.3 Calibration of Sample Canister Pressure Gauge: The dial pressure gauge of each sample canister is certified using standards or equipment traceable to the NIST. Each canister gauge is recertified once per month, or as necessary.

7.3.4 Calibration of the Mercury Thermometer: The mercury thermometer is certified using standards or equipment traceable to the NIST.

7.3.5 Calibration of the Organic Vapor Analyzer: Cylinders of both zero air and $250 \mathrm{ppm}$ isobutylene, certified using standards traceable to NIST, are supplied by the manufacturer of the organic vapor analyzer with a PID, for calibrating the unit. 


\subsection{REFERENCES}

1. U.S. Environmental Protection Agency (U.S. EPA), 1988, Compendium Method TO-14, The Determination of Volatile Organic Compounds (VOC) in Ambient Air Using SUMMA ${ }^{\oplus}$ Passivated Canister Sampling and Gas Chromatographic Analyses, May 1988, Quality Assurance Division, Environmental Monitoring Systems Laboratory, Research Triangle Park, North Carolina.

2. U.S. EPA, 1986, "Test Methods for Evaluating Solid Waste, Physical/Chemical Methods," SW-846, Third Edition, Office of Solid Waste and Emergency Response, Washington, D.C.

3. Oliver, K.D., J.D. Pleil, and W.A. McClenny, 1986, Atmospheric Environment, Vol. 20, No. 7, pp. 1403-1411.

4. Molecke, M.A., 1990a, "Test Plan: WIPP Bin-Scale CH TRU Waste Tests," SAND90-1974, Sandia National Laboratories, Albuquerque, New Mexico.

5. Molecke, M.A., 1990b, "Test Plan: WIPP In-Situ Alcove CH TRU Waste Tests," Sandia National Laboratories, Albuquerque, New Mexico.

6. U.S. Department of Energy (USDOE), 1991a, "Quality Assurance Program Plan for the Waste Isolation Pilot Plant Experimental-Waste Characterization Program," Rev. 1.0, DOE/EM/48063-1, Department of Energy, Office of Waste Operations, Washington, D.C.

7. U.S. Environmental Protection Agency (USEPA), 1990, "Conditional No-Migration Determination for the Department of Energy Waste Isolation Pilot Plant (WIPP)," Title 55, Federal Register, No. 47700 (55 FR 47700) November 14, 1990. 
PROCEDURE 110.3

HEADSPACE GAS SAMPLING OF WIPP EXPERIMENTAL BINS

\subsection{SCOPE AND APPLICATION}

1.1 During the WIPP Test Phase, DOE plans to conduct bin-scale experiments in the WIPP to assess rates and potential for gas generation that may influence the long-term integrity of the repository. At the generator/storage facilities, four to six drums of TRU wastes will be emptied into each test bin and placed in a WIPP standard waste box (SWB). The experimental bins are sampled at the generator storage sites to demonstrate compliance with conditions specified in the No-Migration Determination (NMD) as described in Section 1.3 of the QAPP. This sample of the bin headspace gas is collected within 20 working days prior to TRUPACT-II loading. Bin sampling for assessment of compliance with the NMD requirements is not to be done until all drum data packages per bin have been validated and approved by DOE/WPO.

1.2 This procedure is designed to ensure that representative headspace gas samples, including quality control samples, are consistently collected. The bin samples include the following:

- Headspace gas samples from within the bin

- Field duplicates

- Sampling equipment blanks

- $\quad$ Reference standards.

1.3 The bin headspace gas sampling system is used to recirculate and homogenize bin headspace gas at a prescribed flow rate for a predetermined length of time adequate to recirculate two bin void volumes, thereby making the collection of a representative headspace gas sample possible. Flow rate and time are a function of waste type and packaging configuration. See Table 1 for bin void volumes as a function of waste type.

\subsection{APPARATUS}

2.1 WIPP Experimental Bin: The bins are steel boxes measuring approximately four feet square and three feet tall. The front face of the bin (shown in Figure 1 ) has several valved ports. The two ports used for sampling the headspace gas, GR-1 and GR-2, are equipped with flexible sampling leads. The sampling leads are made of Cajon ${ }^{\oplus} 321$ stainless steel flexible bellows type tubing that has a wall thickness of 0.006 inches. On the ends of these leads are Kevlar filters that prevent radioactive contamination from escaping the bin. The other valves on the bin come without handles to ensure they are not inadvertently opened. 
Revision: 0

Date: August 30, 1991

Table 1. Bin Void Volume as a Function of Waste Type

Waste Type

I (Solidified aqueous or homogeneous inorganics)

II (Solid inorganics)

III $^{\mathrm{b}}$ (Solid organics)

III ${ }^{\mathrm{C}}$ (Solid organics)

IV (Solidified organics)

a Molecke, M.A., 1990a and Nupac, 1989

b Shipping Category III.1 D2

C Shipping Category III.1 D5
Void Volume (L)

550

760

690

890

560 


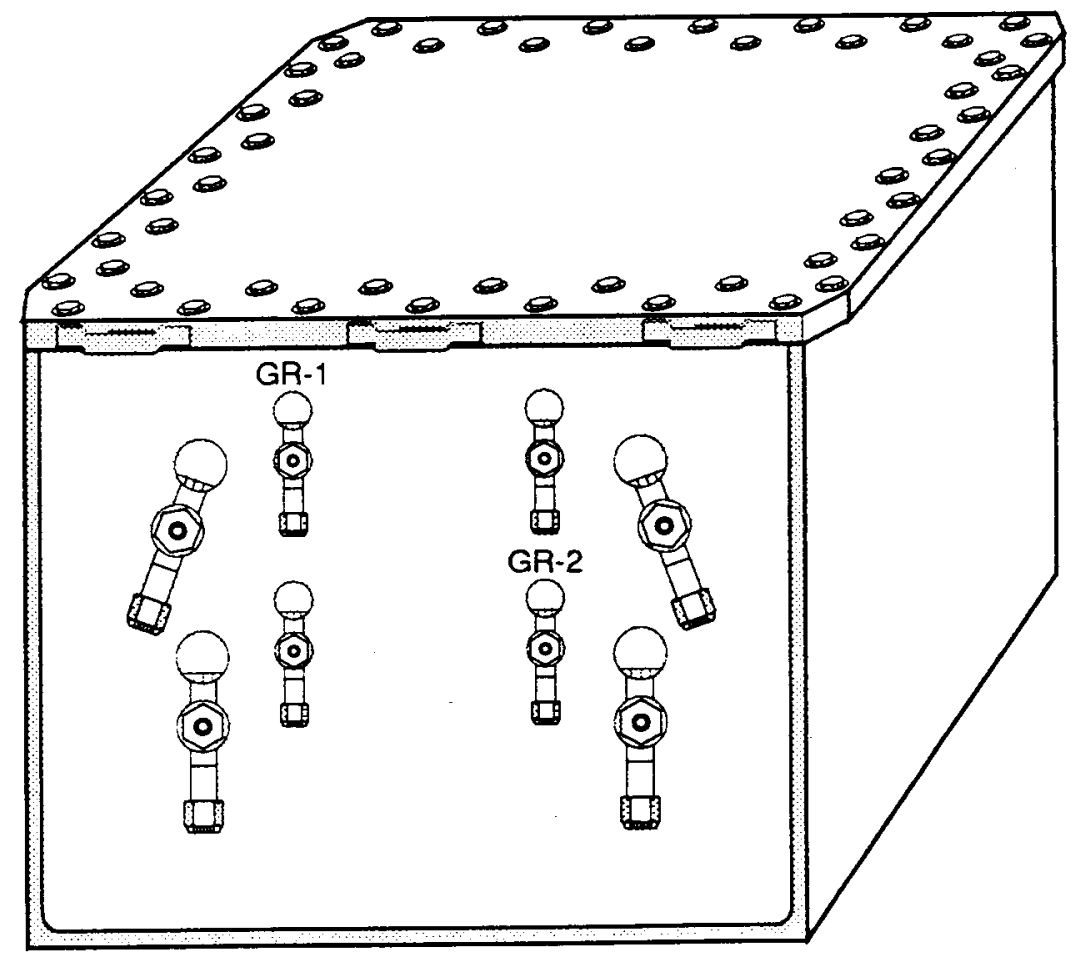

Figure 1. WIPP Experimental Bin 
2.1.1 On the back side of each bin are two filtered breather vents for transportation purposes. The Kevlar filters on these vents are identical to those used on the sampling leads.

2.1.2 Each bin arrives at the sampling site contained inside a standard waste box (SWB). The SWB protects the bin and the associated valves and vents from damage.

2.2 Bin Headspace Gas Sampling System: The bin headspace gas sampling system, shown in Figure 2, is approximately two and one-half feet square and three feet tall, and weighs approximately 134 pounds with two SUMMA ${ }^{\oplus}$ canisters connected. The system consists of an inlet port, an outlet port, a pump, an elapsed time meter, solenoid valves, a pneumatic recirculation loop, flow controlling and flow indication equipment, pressure regulating equipment, and a sample canister(s). The system is contained in a transportable protective housing, mounted on casters, with removable front and rear doors (not shown). The system is secured (as shown in Figure 3) on top of the bin and connected to the bin valves without having to remove the casters.

A schematic of the bin headspace gas sampling system, connected to the bin's flexible sampling leads, is shown in Figure 4. Each component is numbered and described in more detail in Table 2, and in the text below. The system components meet the specifications described as follows:

- An oil free pump (27) for circulating bin headspace gas at a minimum rate of approximately $1 \mathrm{~L} / \mathrm{min}$ and capable of pressurizing the sample canister(s) to a pressure of $15 \pm 2$ psig.

- Flow controlling device (39) capable of regulating pump flow rate at $1.0 \pm 0.2 \mathrm{~L} / \mathrm{min}$.

- Flow meter $(30)$ capable of monitoring gas flow rates of approximately 0 to $3 \mathrm{~L} / \mathrm{min}$ through the recirculation loop.

- Redundant bin pressure regulating equipment (18 through 23), capable of maintaining the internal bin pressure between a minimum of minus 5 psig and a maximum of plus 5 psig.

- Six-liter SUMMA ${ }^{\oplus}$ passivated canister(s) $(43$ and 44$)$ equipped with vacuum/pressure gauges (41 and 42) evacuated to at least $0.05 \mathrm{~mm} \mathrm{Hg}$.

- Flow controlling device (33 and 34 ) on each canister capable of regulating flow to the canister at approximately $100 \mathrm{ml} / \mathrm{min}$.

- All components that come into contact with sample gases are constructed of relatively inert materials such as stainless steel, $V_{i t o n}{ }^{\circ}$, or Teflon ${ }^{\oplus}$. A passivated interior surface on the stainless steel components is recommended.

- Vacuum/pressure gauge(s) $(41$ and 42) for measuring sample canister pressures between 30 inches $\mathrm{Hg}$ and 30 psi. 
Revision: 0

Date: August 30, 1991

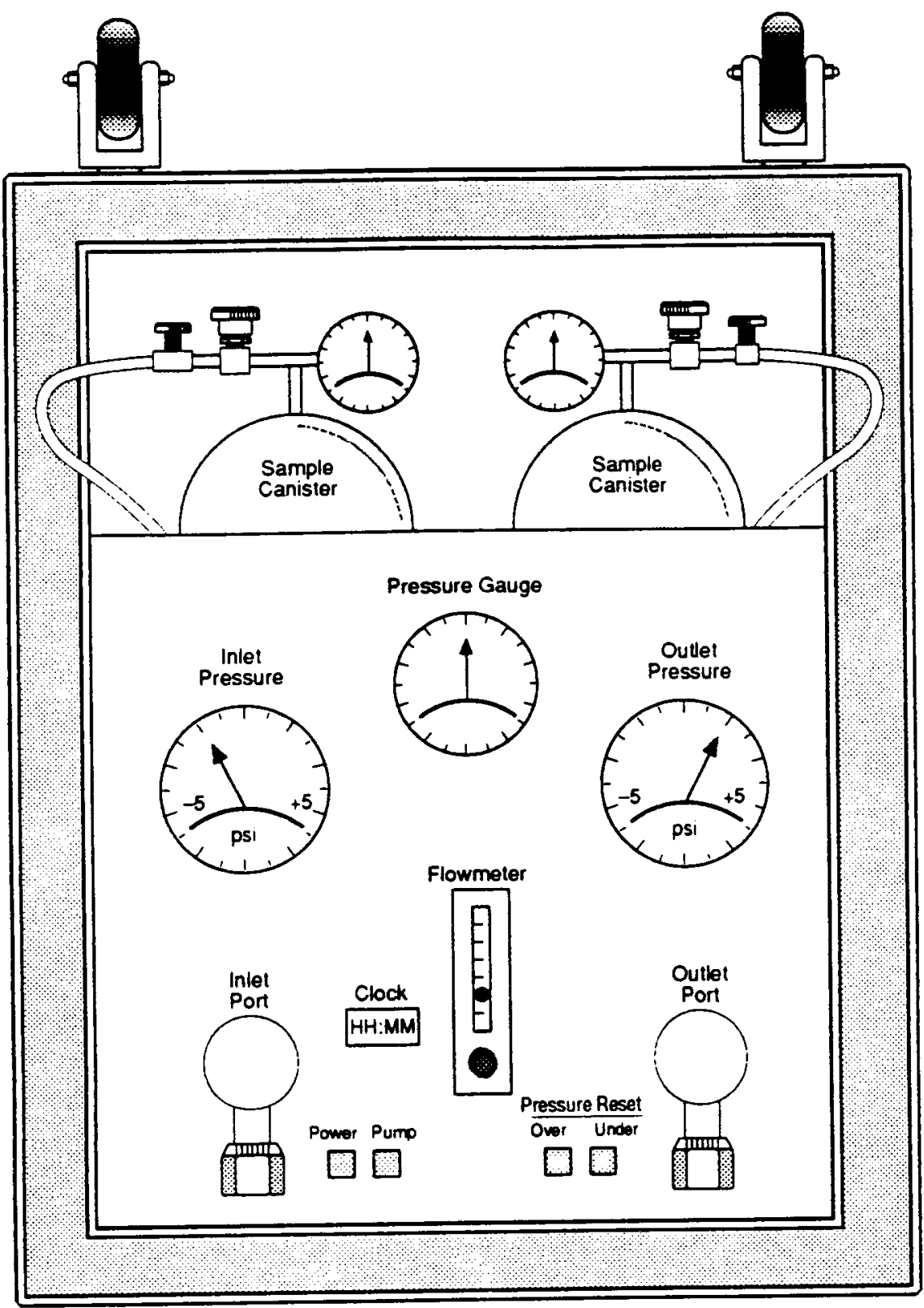

Figure 2. WIPP Experimental Bin Headspace Gas Sampling System 


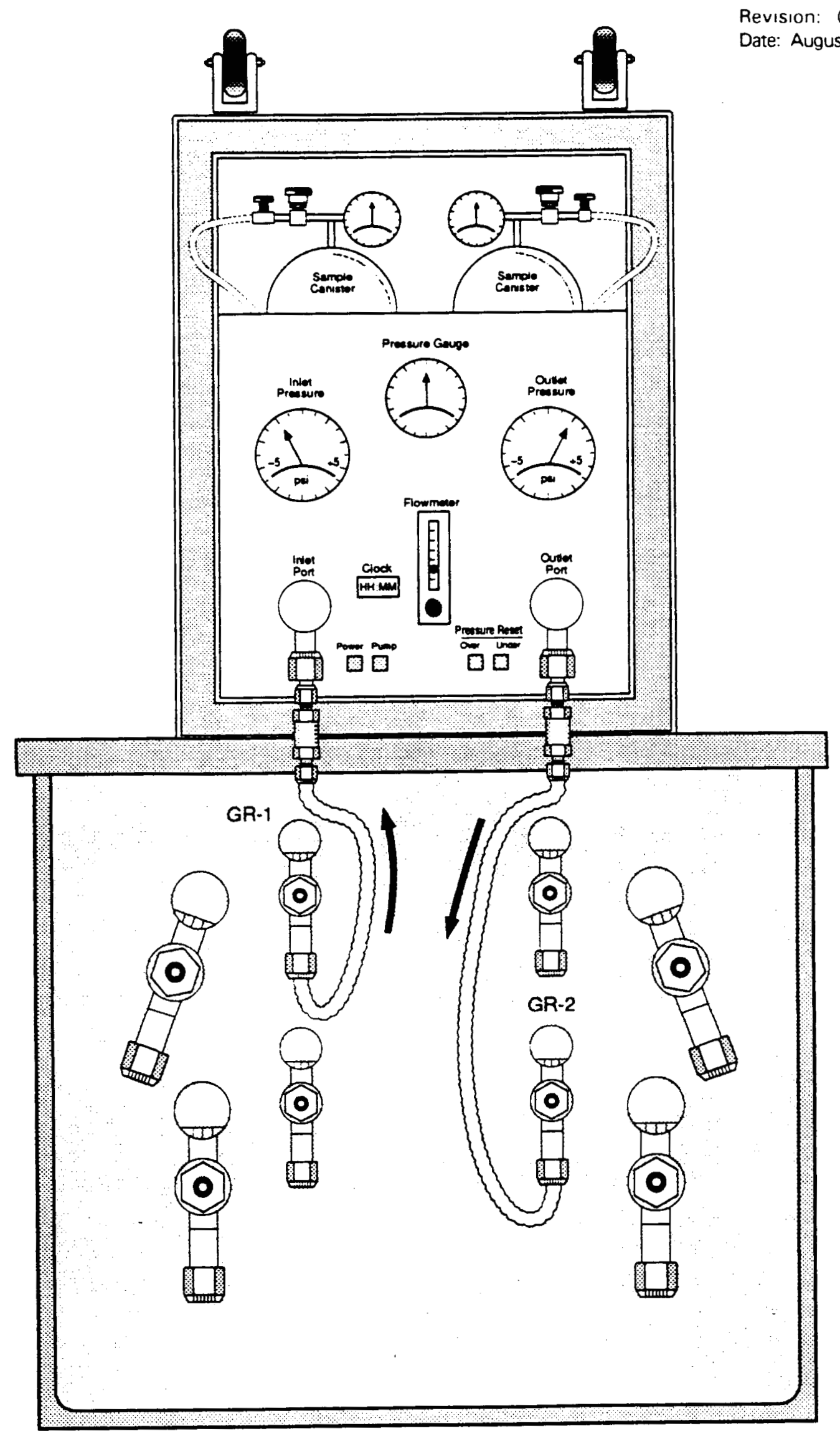

Figure 3. Configuration of WIPP Experimental Bin Headspace Gas Sampling System Attached to the Bin 
Revision: 0

Date: August 30, 1991

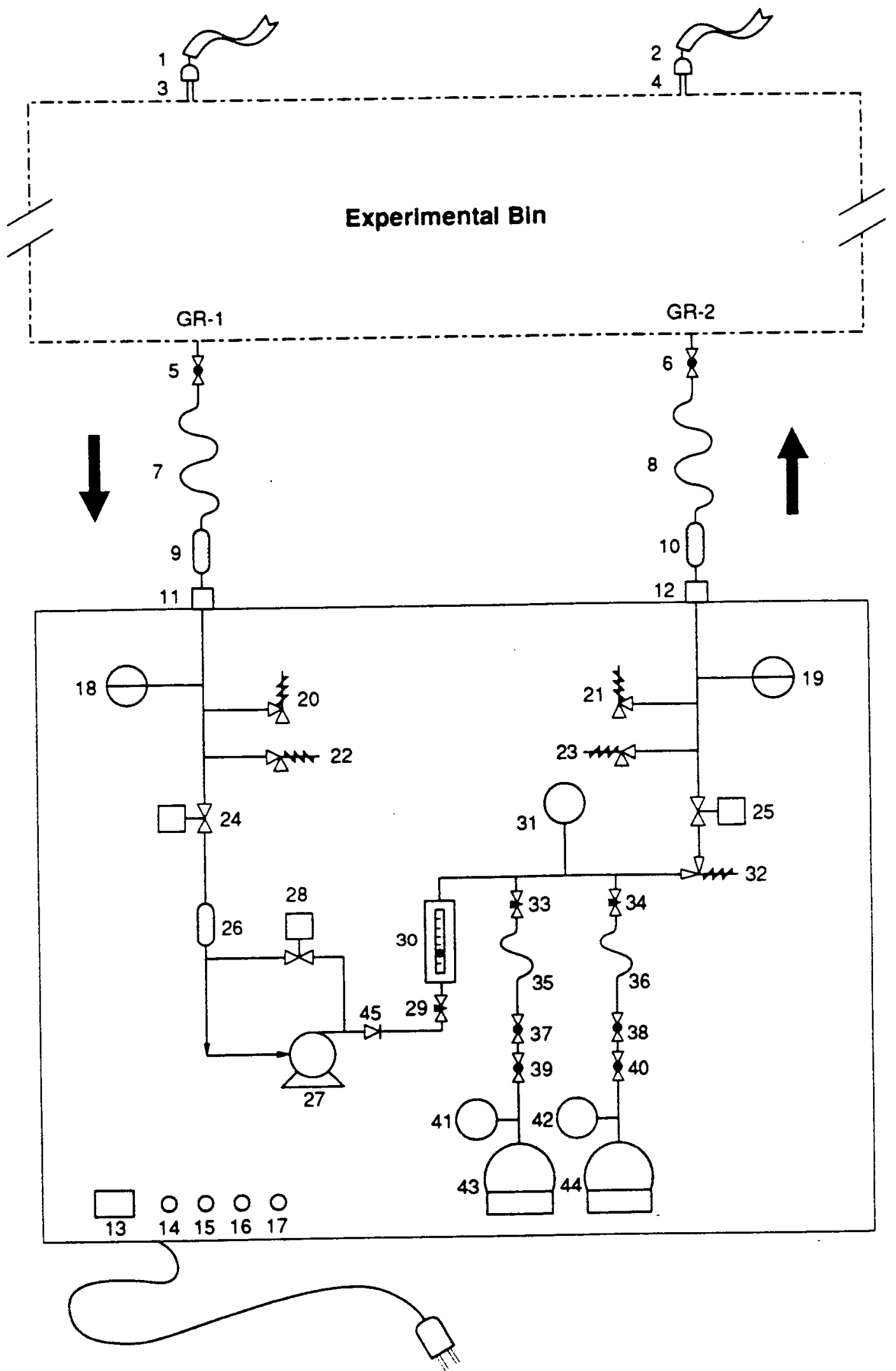

Figure 4. Schematic of the WIPP Experimental Bin Headspace Gas Sampling System 
Table 2. Bin Headspace Gas Sampling System: Listing of Major Components

\begin{tabular}{|c|c|c|}
\hline $\begin{array}{c}\text { Component } \\
\text { No. }\end{array}$ & Component Description & Purpose \\
\hline 1 & $\begin{array}{l}\text { Bin breather filter cap with } \\
\text { streamer }\end{array}$ & Seal bin during sampling operations \\
\hline 2 & $\begin{array}{l}\text { Bin breather filter cap with } \\
\text { streamer }\end{array}$ & Seal bin during sampling operations \\
\hline 3 & Bin breather filter & Bin vent during transportation \\
\hline 4 & Bin breather filter & Bin vent during transportation \\
\hline 5 & $1 / 2 "$ bin port valve (GR-1) & Allows access to bin for sampling \\
\hline 6 & $1 / 2^{n}$ bin port valve (GR-2) & Allows access to bin for sampling \\
\hline 7 & $\begin{array}{l}\text { Bin sampling lead (flexible } \\
\text { bellows tubing) }\end{array}$ & Allows access to bin for sampling \\
\hline 8 & $\begin{array}{l}\text { Bin sampling lead (flexible } \\
\text { bellows tubing) }\end{array}$ & Allows access to bin for sampling \\
\hline 9 & Bin filter (0.3 micron Kevlar & Radiological control barrier \\
\hline 10 & Bin filter (0.3 micron Kevlar() & Radiological control barrier \\
\hline 11 & $\begin{array}{l}1 / 2^{n} \text { sampling system inlet } \\
\text { port }\end{array}$ & Bin headspace gas enters sampling system here \\
\hline 12 & $\begin{array}{l}1 / 2^{n} \text { sampling system outlet } \\
\text { port }\end{array}$ & Bin headspace gas leaves sampling system here \\
\hline 13 & Clock (hour and minutes) & Measure elapsed gas recirculation time \\
\hline 14 & $\begin{array}{l}\text { Power switch and indicator } \\
\text { light }(110 \vee \mathrm{VAC})\end{array}$ & Main power switch, energizes sampling system \\
\hline 15 & $\begin{array}{l}\text { Pump power switch }(110 \\
\text { VAC) }\end{array}$ & Allows for independent control of pump power \\
\hline 16 & $\begin{array}{l}\text { Over pressure indicator light } \\
\text { and reset switch }\end{array}$ & $\begin{array}{l}\text { Indicates if bin has been over pressurized during } \\
\text { sampling }\end{array}$ \\
\hline 17 & $\begin{array}{l}\text { Under pressure indicator light } \\
\text { and reset switch }\end{array}$ & $\begin{array}{l}\text { Indicates if bin has been under pressurized } \\
\text { during sampling }\end{array}$ \\
\hline 18 & $\begin{array}{l}\text { Pressure gauge/switch with } \\
\text { high/low set points }\end{array}$ & $\begin{array}{l}\text { Indicates sampling system inlet line pressure } \\
\text { and cuts power to system if inlet line pressure } \\
\text { falls below }-2.5 \text { psig }\end{array}$ \\
\hline 19 & $\begin{array}{l}\text { Pressure gauge/switch with } \\
\text { high/low set points }\end{array}$ & $\begin{array}{l}\text { Indicates sampling system outlet line pressure } \\
\text { and cuts power to system if outlet line pressure } \\
\text { rises above } 2.5 \text { psig }\end{array}$ \\
\hline
\end{tabular}


Table 2. Bin Headspace Gas Sampling System: Listing of Major Components (Continued)

\begin{tabular}{|c|c|c|}
\hline $\begin{array}{c}\text { Component } \\
\text { No. }\end{array}$ & Component Description & Purpose \\
\hline 20 & $\begin{array}{l}\text { Mechanical vacuum relief } \\
\text { valve }\end{array}$ & $\begin{array}{l}\text { Automatically opens to relieve system inlet line } \\
\text { if vacuum exceeds }-5 \text { psig }\end{array}$ \\
\hline 21 & $\begin{array}{l}\text { Mechanical vacuum relief } \\
\text { valve }\end{array}$ & $\begin{array}{l}\text { Automatically opens to relieve system outlet } \\
\text { line if vacuum exceeds }-5 \text { psig }\end{array}$ \\
\hline 22 & $\begin{array}{l}\text { Mechanical pressure relief } \\
\text { valve }\end{array}$ & $\begin{array}{l}\text { Automatically opens to relieve system inlet line } \\
\text { if pressure exceeds } 5 \text { psig }\end{array}$ \\
\hline 23 & $\begin{array}{l}\text { Mechanical pressure relief } \\
\text { valve }\end{array}$ & $\begin{array}{l}\text { Automatically opens to relieve system outlet } \\
\text { line if pressure exceeds } 5 \text { psig }\end{array}$ \\
\hline 24 & Inlet solenoid valve & $\begin{array}{l}\text { Isolates bin sampling system during } \\
\text { transportation and handling }\end{array}$ \\
\hline 25 & Outlet solenoid valve & $\begin{array}{l}\text { Isolates bin sampling system during } \\
\text { transportation and handling }\end{array}$ \\
\hline 26 & $\begin{array}{l}\text { Sampling system inlet filter } \\
(0.5 \text { micron sintered stainless } \\
\text { steel) }\end{array}$ & Radiological assessment filter \\
\hline 27 & Vacuum/compressor pump & $\begin{array}{l}\text { Recirculates bin headspace gas prior to } \\
\text { sampling and pressurizes headspace gas } \\
\text { samples to } 15 \text { psig }\end{array}$ \\
\hline 28 & $\begin{array}{l}\text { Pump head bleed solenoid } \\
\text { valve }\end{array}$ & $\begin{array}{l}\text { Bleeds pump head pressure to the system inlet } \\
\text { line whenever the pump is off }\end{array}$ \\
\hline 29 & Needle valve & Controls bin headspace gas recirculation rate \\
\hline 30 & Flow meter & Indicates bin headspace gas recirculation rate \\
\hline 31 & $\begin{array}{l}\text { Pressure gauge } \\
(0-30 \text { psig) } \\
\end{array}$ & $\begin{array}{l}\text { Indicates pressure in the pneumatic line } \\
\text { connecting the sample canisters }\end{array}$ \\
\hline 32 & Pressure regulating valve & $\begin{array}{l}\text { Controls the pressure in the pneumatic line } \\
\text { connecting the sample canisters }\end{array}$ \\
\hline 33 & Sample canister needle valve & Controls rate at which sample canister fills \\
\hline 34 & Sample canister needle valve & Controls rate at which sample canister fills \\
\hline 35 & $\begin{array}{l}\text { Stainless steel flexible hose } \\
\text { lined with Teflon } \\
\end{array}$ & $\begin{array}{l}\text { Connects sample canister to the recirculation } \\
\text { loop }\end{array}$ \\
\hline 36 & $\begin{array}{l}\text { Stainless steel flexible hose } \\
\text { lined with Teflon }\end{array}$ & $\begin{array}{l}\text { Connects sample canister to the recirculation } \\
\text { loop }\end{array}$ \\
\hline 37 & Plug valve & Isolates sample canister from sampling system \\
\hline 38 & Plug valve & Isolates sample canister from sampling system \\
\hline
\end{tabular}


Table 2. Bin Headspace Gas Sampling System: Listing of Major Components (Continued)

\begin{tabular}{||c|l|l||}
\hline $\begin{array}{c}\text { Component } \\
\text { No. }\end{array}$ & \multicolumn{1}{|c|}{ Component Description } & \multicolumn{1}{|c|}{ Purpose } \\
\hline 39 & Sample canister bellows valve & Isolates sample canister from ambient air \\
\hline 40 & Sample canister bellows valve & Isolates sample canister from ambient air \\
\hline 41 & $\begin{array}{l}\text { Sample canister } \\
\text { vacuum/pressure gauge }\end{array}$ & Indicates sample canister vacuum/pressure \\
\hline 42 & $\begin{array}{l}\text { Sample canister } \\
\text { vacuum/pressure gauge }\end{array}$ & Indicates sample canister vacuum/pressure \\
\hline 43 & $\begin{array}{l}\text { Six-liter SUMMA } \\
\text { sample canister }\end{array}$ & Sample container \\
\hline 44 & $\begin{array}{l}\text { Six-liter SUMMA } \\
\text { sample canister }\end{array}$ & Sample container \\
\hline 45 & Check valve & $\begin{array}{l}\text { Prevents back flow of recirculating gas and loss } \\
\text { of sample on over/under pressure trip }\end{array}$ \\
\hline
\end{tabular}


- Ambient pressure sensor with an approximate range of 500 to $800 \mathrm{~mm} \mathrm{Hg}$ and a resolution of $1 \mathrm{~mm} \mathrm{Hg}$. This sensor may be mounted on the sampling system or in the sampling area.

- Ambient temperature sensor with an approximate range of $-30^{\circ} \mathrm{C}$ to $50^{\circ} \mathrm{C}$ and a resolution of $1^{\circ} \mathrm{C}$. This sensor may be mounted on the sampling system in the sampling area.

- A battery backed-up clock (elapsed time meter) for monitoring pump operating time and displaying hours and minutes.

- A pressure gauge (31) with a range of at least 0 to 30 psi for monitoring the pressure in the pneumatic lines connecting the sample canisters.

- A pressure regulating valve (32) capable of maintaining $15 \pm 2$ psi pressure in the lines connecting the sample canisters.

\subsection{REAGENTS}

Not Applicable.

\subsection{SAMPLE HANDLING AND PROCEDURES}

4.1 Procedures for Cleaning the Sampling System: The procedures for cleaning the sampling system are included to ensure a representative sample is collected by preventing cross-contamination between samples. These procedures maintain quality control of the sampling process. Procedure 4.1.1 is a method for cleaning the individual system components as they are collected and assembled. Procedure 4.1.2 describes the routine system leak check. Procedure 4.1.3 describes the routine sampling system cleaning conducted between sampling events to prevent cross contamination. In the event Procedure 4.1.3 fails to properly clean the sampling system, as indicated by the equipment blank, the system is disassembled and cleaned as described in Procedure 4.1.1. Procedure 4.1.4 describes the method for cleaning sample canisters.

\subsubsection{Initial Sampling System Cleaning: Upon receipt from the various manufacturers} and prior to assembly, the surfaces of the sampling system components that will come into contact with sample gases are inspected and thoroughly cleaned using this procedure. Parts received free of contamination, as stated or certified by the manufacturers, may be exempted from this cleaning procedure at the discretion of the sampling personnel.

1. Rinse all nonmetallic components of the sampling system with HPLC-grade deionized water and dry in an oven at $50^{\circ} \mathrm{C}$ for at least 12 hours. An evacuated oven is preferable. 
2. Place stainless steel parts and fittings in a beaker of reagent-grade hexane in an ultrasonic bath for 15 minutes. Repeat this procedure with reagent-grade methanol. Rinse the stainless steel parts with HPLC-grade deionized water and dry in an oven at $100^{\circ} \mathrm{C}$ for at least 12 hours. Stainless steel parts and fittings may also be cleaned by assembling and forcing steam through the assembly.

4.1.2 Routine Sampling System Leak Check: This procedure describes the steps for detecting and repairing system leaks once the sampling system has been assembled and prior to attaching sample canisters. See Figure 4 for the location of the numbered components and Figure 5 for the location of labeled purge manifold components.

1. With power turned off to the sampling system, remove the back cover of the sampling system case and access the pump (27). Remove the 1/4" flexible tubing from the outlet of the sampling system pump.

2. Attach a valve to this line and attach the line and valve to a clean, regulated cylinder of ultra high purity (UHP) nitrogen or zero air. Close the valve and set the regulator to deliver 20 psig.

3. Slowly open the valve. Monitor the line pressure on the sampling system pressure gauge (31). When the pressure gauge reads 20 psi, close the valve. The pressure will drop to the pressure as set on the pressure regulating valve (32), approximately $15 \mathrm{psig}$.

4. Allow the pressure to stabilize after the pressure regulating valve seats.

5. Monitor the leak rate by observing the sampling system pressure gauge (31) on the control panel. To pass the leak test, the sampling system pressure must remain constant for 30 minutes.

6. If leaks are detected (indicated by the pressure dropping below the set pressure), then tighten fittings or otherwise isolate and repair the leaking components until an acceptable leak rate can be achieved.

\section{CAUTION: Do not over-tighten fittings.}

NOTE: Some components of the sampling system pressurized line, i.e., the pump (27) and bypass solenoid valve $(28)$ are not tested by this procedure. The pump is not designed to be leak tight against a static back pressure when the pump is not operating. Since the pump will be running during sample collection, back-streaming through the pump is not considered an important source of leaks.

4.1.3 Routine Sampling System Cleaning: The following procedure is used to clean the bin headspace gas sampling system between each sampling operation. This procedure is performed in the analytical laboratory prior to shipment of the sampling system to the sampling site.

1. Install the sampling system purge manifold as shown in Figure 5 . Use new 1/2" gaskets when attaching $V C R^{\oplus}$ fittings to the sampling system inlet and outlet ports.

NOTE: Use cotton gloves when installing gaskets to prevent contamination and ensure a good seal. 
Revision: 0

Date: August 30, 1991

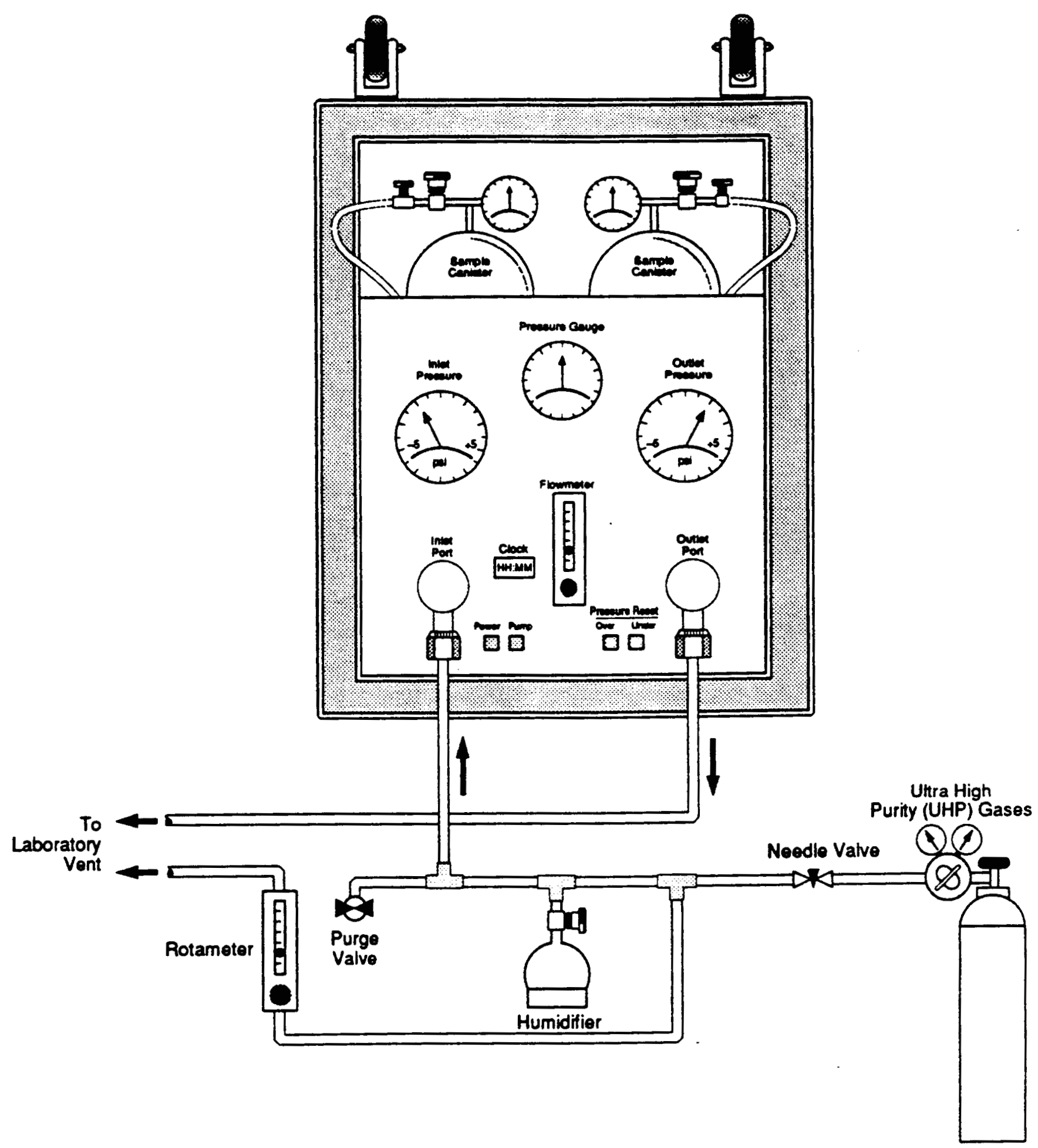

Figure 5. Schematic of the Bin Sampling System Attached to Laboratory Purge Manifold 
2. Connect a regulated cylinder of UHP nitrogen or zero air to the inlet of the precision needle valve. Open the valve to the humidifier.

3. Set the regulator to deliver 20 psig.

4. Open the rotameter valve and then slowly adjust the needle valve until the rotameter indicates 1.5 to $2.0 \mathrm{~L} / \mathrm{min}$. flow rate.

5. Open plug valves (37) and (38).

6. Start the sampling system pump.

7. Adjust the needle valve to ensure that rotameter indicates excess flow. The sampling system is now being purged.

8. Purge the sampling system for 1 hour.

NOTE: At this point the purge manifold system can be used for collecting an equipment blank in accordance with the procedure described in Section 4.2.3. After the equipment blank collection, a reference standard sample may be collected in accordance with the procedure described in Section 4.2.4.

9. Close valve (37) and (38).

10. Turn off the power to the sampling system.

11. Close the supply valve on the cylinder of UHP nitrogen or zero air and disconnect the sampling system purge manifold.

12. Cap inlet and outlet ports (11) and (12) using VCR caps and new gaskets.

13. Attach a clean evacuated sample canister to either plug valve (37) or (38) for single sample collection and to both plug valves for duplicate sampling.

\subsubsection{Sample Canister Cleaning: Follow the method specified in Guidance Manual} Procedure 210.1.

4.2 Procedure for Collecting Headspace Gas and Quality Control Samples: Headspace gas and quality control samples collected using the sampling procedures described here comply with the headspace gas sampling data quality and quality assurance objectives (see QAPP Sections 1.4 and 3.3).

4.2.1 Headspace Gas Sample Collection: The following procedure describes the use of the bin headspace gas sampling system for collection of headspace gas samples from within WIPP experimental bins. Refer to Figure 4 for location of numbered components.

CAUTION: Appropriate site radiological controls must be established prior to and during sampling operations. 


\section{System Setup}

1. Remove the front case cover from the sampling system.

2. Place the sampling system on top of the WIPP Experimental Bin with the casters pointing upwards. Position the sampling system so its control panel is lined up with, and centered above, the valved bin face.

3. Secure the sampling system to the top of the WiPP Experimental Bin using tie downs.

4. Visually check the system for damage. Verify that the pressure switch/gauges (18) and (19), pressure gauge (31), and rotameter (30) have current calibration tags. Also verify that the sampling system has been leak checked and cleaned according to procedures 4.1.2 and 4.1.3, respectively. If any component shows signs of damage or has an expired or missing certification tag, return the system to the lab for recertification.

5. Check that the sample canister vacuum gauge (41) or (42) indicates a vacuum of 30 inches $\mathrm{Hg}$. If the canister gauge indicates a vacuum less than this, return the sampling system to the lab for recertification.

6. Carefully remove the packing material from flexible bin sampling leads (7) and (8). Handle the leads gently to avoid stretching or kinking.

7. Put on a pair of clean cotton glove liners, or cotton gloves and remove the caps and gaskets from inlet and outlet ports (11) and (12).

8. Connect bin filter (9) to the sampling system inlet port (11) installing a new gasket.

NOTE: New gaskets are supplied with the sampling system in a resealable envelope.

9. Repeat step 8 for bin filter (10) and outlet port (12).

10. Open bin valves (5) and (6) completely.

11. Install caps (1) and (2) on bin breather filters (3) and (4). The caps are provided with the sampling system and identified by their attached yellow streamers.

12. On pressure gauge/switches (18) and (19), adjust the low set point to -2.5 psi and the high set point to 2.5 psi.

13. Plug the sampling system into $110 \mathrm{VAC}$ power.

14. If the power switch (14) does not light up, press the switch to turn on power to the system. If pump switch (15) lights up, press it to turn off the pump.

NOTE: When the power to the system is first turned on, reset switches (16) and (17) should light up in addition to the power switch. Failure of one of these switches to light indicates that either a light bulb has burned out or there is an electrical problem. Return the system to the lab for repair and recertification.

15. Press the over pressure and under pressure reset switches (16) and (17) to reset the system. The switch lights should turn off. 
16. Press the red button on the control panel clock (13) to reset the clock to zero. Check that the clock is displaying elapsed time from zero after it is reset. The clock displays both hours and minutes.

17. Activate the system's recirculation pump (27) by pressing pump switch (15). The green pump switch should light when the pump is turned on.

18. Adjust needle valve (29), if necessary, until flow meter (30) indicates a flow of $1.0 \pm$ 0.2 liters per minute. Consult the calibration data that identifies what value on the rotameter corresponds to 1.0 liter per minute.

19. After a few minutes of operating time, check that pressure gauge (31) indicates $15 \pm$ 2 psig. If pressure gauge (31) does not indicate this pressure, turn off pump switch (15), power switch (14), and return the system to the laboratory for recertification.

NOTE: If at any time the system is automatically shut down due to an over or under pressure condition, do not restart the system. Return the system to the laboratory for sample analysis, if possible, system cleaning and recertification.

\section{Recirculation}

20. The system is set to recirculate the bin gases at a minimum flow rate of $1.0 \pm 0.2$ liters per minute. The system operates at this flowrate until two bin void volumes have been recirculated. See Table 1 for bin void volumes as a function of waste type. At 4-hour intervals, check the system to ensure that it is operating properly.

NOTE: If necessary, the flow rate (30) can be readjusted (29) during the recirculation period to maintain the $1.0 \pm 0.2$ liter per minute flow. Record the time of the adjustment and the flow rate both before and after the adjustment.

\section{Sampling}

21. After the sampling system has recirculated two bin void volumes, verify that the system's pressure gauge $(31)$ indicates $15 \pm 2$ psig. If not, log the pressure and proceed with step 22.

22. Open plug valve (37 or 38$)$ to which the canister is attached.

23. Again check canister pressure gauge (41 or 42 ) to ensure that it indicates 30 inches $\mathrm{Hg}$. Record the reading on the canister tag.

NOTE: If the pressures indicated on pressure gauge (41) or (42) do not agree with the pressure last recorded on its canister tag, a canister leak is suspect and sample collection is not advised.

24. Open canister valve (39) or (40). Needle valve (33) or (34) is preset in the laboratory to deliver bin gas to the attached sample canister at a flow rate of $100 \mathrm{ml} / \mathrm{min} \pm 10$ $\mathrm{ml} / \mathrm{min}$.

25. The pressure gauge (31) indication will decrease while the canister is being filled. When pressure gauge (31) again indicates to $15 \pm 2$ psig and canister gauge (41) or (42) also indicates approximately 15 psi, close canister valve (39) or (40) and then close plug valve (37) or (38). 
26. Record the date, time, canister gauge pressure, ambient pressure, and ambient temperature on the sample canister tag (see QAPP Section 5, Sample Canister Tag).

27. Note on the OC sample record that the sample canister(s) contains a headspace gas sample (see QAPP Section 5, OC Sample Record Form).

\section{System Shut Down}

28. Shut off the pump (27) by pressing the pump switch (15).

29. Shut off the power by pressing power switch (14) and unplug the system.

30. Wearing clean cotton glove linens or cotton gloves, slowly remove caps (1) and (3) from bin breather filters (3) and (4). Discard the used gaskets and store the caps.

31. Close bin valves (5) and (6) and disconnect bin filters (9) and (10) from sampling system inlet port (11) and outlet port (12). Discard the used gaskets.

32. After bin filters (9) and (10) have been disconnected from the sampling system, carefully repackage and secure them for shipping.

33. The sample canister(s) is not removed from the sampling system. Rather, the whole sampling system, along with the canister(s) still attached, is sent to the analysis lab. Reconnect the caps and gaskets that were removed from the sampling system during Step 7 .

34. Remove the tie downs that were used to secure the sampling system to the top of the bin.

35. Carefully set the sampling system on its casters on the floor and secure the front case cover.

4.2.2 Field Duplicate Collection: Field duplicates are collected either simultaneously or sequentially by following the procedures in Section 4.2.1 and opening canister valves (39) and (40) at the same time or opening the second canister valve at some predetermined time after the first canister has filled (see Step 24). These samples are used to assess the precision of the sampling and analytical process. Field duplicates are collected at a frequency of one per bin for the first two bins loaded, then one per five bins, thereafter.

4.2.3 Equipment Blank Collection: The following procedure describes the use of the bin sampling system for collection of equipment blanks. These samples are used to assess the cleanliness of the sampling system. An equipment blank is collected and analyzed prior to each use of the sampling system. Refer to Figure 4 for location of numbered components and Figure 5 for the location of labeled purge manifold components.

1. Verify that the bin sampling system has been cleaned in accordance with Steps 1 through 8 of the procedure described in Section 4.1.3. 
Revision: 0

Date: August 30, 1991

2. Attach a clean, evacuated sample canister to plug valve (37) or (38). Close the plug valve to which a canister is not attached.

3. Open the canister valve. Monitor the sample canister pressure gauge for canister pressure.

4. When the sample canister pressure gauge indicates the same pressure as that indicated on the sampling system pressure gauge (approximately $15 \mathrm{psig}$ ), close the sample canister valve and plug valve. Remove the sample canister.

5. Follow Steps 9 through 13 of the procedure described in Section 4.1.3 to shut the system down.

6. Record the date, time, canister gauge pressure, ambient pressure, and ambient temperature on the sample canister tag (see QAPP Section 5, Sample Canister Tag).

7. Note on the $\mathrm{OC}$ sample record that the sample canister(s) contains an equipment blank (see QAPP Section 5, OC Sample Record Form).

4.2.4 Reference Standard Collection: The following procedure describes the use of the bin sampling system for collection of reference standards. These samples are used to assess the accuracy of the sampling and analysis process. A reference standard is collected and analyzed prior to first use of the sampling equipment, then at a frequency of one per bin for the first two bins loaded, followed by a frequency of one per five bins, thereafter. Refer to Figure 4 for location of numbered components and Figure 5 for the location of purge manifold components.

1. Verify that the bin sampling system has been cleaned in accordance with Steps 1 through 8 of the procedure described in Section 4.1.3.

2. Close plug valves (37) and (38).

3. Turn off the power (15) to the sampling system pump (27).

4. Replace the nitrogen or zero air cylinder used during cleaning with a cylinder of reference standard gas and set the gas flow rate to 1.5 to $2.0 \mathrm{~L} / \mathrm{min}$ as indicated by the rotameter.

5. Turn on the power (15) to the sampling system pump (27).

6. Open plug valves (37) and (38) and purge the sampling system with reference standard gas for approximately 5 minutes.

7. Attach a clean, evacuated sample canister to a plug valve (37) or (38) and close the other plug valve.

8. Open the canister valve. Monitor the sample canister pressure gauge for canister pressure.

9. When the sample canister pressure gauge indicates the same pressure as that indicated on the sampling system pressure gauge (approximately 15 psi), close the sample canister valve. 
10. Open the purge manifold valve and close the reference standard gas supply valve.

11. Remove the sample canister.

12. Record the date, time, canister gauge pressure, ambient pressure, and ambient temperature on the sample canister tag (see QAPP Section 5, Sample Canister Tag).

13. Note on the $Q C$ sample record that the sample canister(s) contains a reference standard sample (see QAPP Section 5, OC Sample Record Form).

14. Reconnect the UHP nitrogen or zero air cylinder to the purge manifold and clean the bin sampling system in accordance with the procedure described in Section 4.1.3.

\subsection{CALCULATIONS}

5.1 See Section 5.0 of Procedure 110.1 for a discussion of calculations to compute the expected sample canister gauge pressure change resulting from ambient temperature and pressure changes.

\subsection{ANALYTICAL PROCEDURE}

6.1 The procedures for analysis of VOCs and gases in the headspace gas of waste containers are provided as Procedures 430.1, 430.2, and 440.1 for VOCs, and as 510.1, 520.1, and 530.1 for gases. The procedure for determining the flammability of the bin headspace gas is provided as Procedure 810.1 .

\subsection{QUALITY CONTROL FOR BIN HEADSPACE GAS SAMPLES}

7.1 Quality control measures include cleaning and leak checking the bin sampling system. The system is cleaned by purging with humidified zero air or UHP nitrogen. After purging, a sample of zero air is collected through the system and analyzed for the Program required analytes. System cleanliness may also be checked by purging with zero air and monitoring the zero air with a photoionization detector.

7.2 The system is also checked for leaks after each cleaning and prior to use. This is performed by pressurizing the system with zero air or UHP nitrogen and checking for leaks by monitoring the internal pressure or performing a soap bubble-type check.

7.3 The field OC samples collected during bin headspace gas sampling operations include equipment blanks, reference standards, and field duplicates. Field blanks are not required during bin 
Revision: 0

Date: August 30, 1991

sampling operations since the bin sampling equipment is carefully sealed to the bin prior to sample collection, thus preventing ambient air intrusion. Reference standards and field duplicates are collected in accordance with the frequencies specified in Table 9-5 and 9-6 of the QAPP. A reference standard (pressurized cylinder gas containing known analytes at known concentrations) is collected using the bin headspace gas sampling apparatus. The results of the analysis of this sample indicate the efficiency of sampling and analytical recovery. Provided the pressure differential across the bin does not exceed \pm 5 psig, field duplicates can be collected.

7.4 A bin headspace gas sample is obtained from $100 \%$ of the bins to be used in the WIPP bin-scale test. Sample holding times and storage conditions are specified in Table 4-1 of the QAPP.

7.5 Consistent use and application of uniform procedures and equipment should ensure that bin headspace gas sampling operations are comparable at the different sampling sites.

7.6 Specific headspace gas sampling steps to ensure that bin headspace gas samples are representative include:

- Recirculation of the headspace gases to achieve homogeneity prior to sampling

- Canister cleaning and leak check

- Equipment cleaning and leak check

- Use of sample canisters with passivated internal surfaces

- Collection of small volume samples: low sample volume to available headspace gas volume ratio

- Careful pressure regulation and flow control

- Performance audits

- Collection of bin headspace gas sampling equipment blanks, reference standards, and field duplicates.

7.7 Quality Control for Pressure and Temperature Measurements: This section pertains to the calibration and certification of equipment and instrumentation used for temperature and pressure measurements. All information related to the calibrations of the pressure and temperatures sensors is recorded.

7.7.1 Certification Check of the Ambient Pressure Sensor: The Fortin barometer is certified by the manufacturer using standards or equipment traceable to the NIST. The pressure 
indication of the barometer is corrected in accordance with temperature indicated by a dual-scale thermometer attached to its casing, if necessary.

7.7.2 Calibration of Sample Canister Pressure Gauge: The dial pressure gauge of each sample canister is certified using standards or equipment traceable to the NIST. Each canister gauge is recertified once per month, or as necessary.

7.7.3 Calibration of the Mercury Thermometer: The mercury thermometer is certified using standards or equipment traceable to the NIST.

7.7.4 Calibration of the Rotameter: The rotameter is certified using standards or equipment traceable to the NIST while operating in a gas stream which is at a pressure of $15 \mathrm{psig.} \mathrm{It}$ is recommended to calibrate the rotameter by connecting the NIST-traceable flow device to the sampling system outlet port (12) while the system is pumping humidified zero air.

7.7.5 Calibration of the Pressure Relief Valves: The pressure relief valves are certified using standards or equipment traceable to the NIST, NBIC, or tested in accordance with ASME Section VIII, PTC 25.2.

7.7.6 Calibration of the Pressure Gauge/Switch: The pressure gauge/switch is certified with standards or equipment traceable to the NIST.

7.7.7 Calibration of the Sampling System Pressure Gauge: The sampling system pressure gauge is certified with standards or equipment traceable to the NIST.

\subsection{REFERENCES}

1. U.S. Environmental Protection Agency IU.S. EPA), 1988, Compendium Method TO-14, The Determination of Volatile Organic Compounds (VOC) in Ambient Air Using SUMMA ${ }^{\circ}$ Passivated Canister Sampling and Gas Chromatographic Analyses, May 1988, Quality Assurance Division, Environmental Monitoring Systems Laboratory, Research Triangle Park, North Carolina.

2. U.S. EPA, 1986, "Test Methods for Evaluating Solid Waste, Physical/Chemical Methods," SW846, Third Edition, Office of Solid Waste and Emergency Response, Washington, D.C.

3. Oliver, K.D., J.D. Pleil, and W.A. McClenny, 1986, Atmospheric Environment, Vol. 20, No. 7, pp. 1403-1411.

4. Molecke, M.A., 1990a, "Test Plan: WIPP Bin-Scale CH TRU Waste Tests," SAND90-1974, Sandia National Laboratories, Albuquerque, New Mexico. 
5. Molecke, M.A., 1990b, "Test Plan: WIPP In Situ Alcove CH TRU Waste Tests," Sandia National Laboratories, Albuquerque, New Mexico.

6. U.S. Department of Energy (U.S. DOE), 1991a, "Quality Assurance Program Plan for the Waste Isolation Pilot Plant Experimental-Waste Characterization Program," Rev. 1.0, DOE/EM/48063-1, Department of Energy, Office of Waste Operations, Washington, D.C.

7. U.S. EPA, 1990, "Conditional No-Migration Determination for the Department of Energy Waste Isolation Pilot Plant (WIPP)," Title 55, Federal Register, No. 47700 (55 FR 4700), November 14, 1990.

8. Nupac, 1989, "Safety Analysis Report for the TRUPACT-Il Shipping Package", Nuclear Packaging, Inc., Federal Way, WA. 


\subsection{CLEANING}

This Guidance Manual contains the following procedure for cleaning and pressure testing sample containers:

Procedure 210.1 provides a procedure for cleaning and pressure certifying SUMMA passivated stainless steel canisters. Prior to cleaning, canisters undergo a positive pressure leak test. Failure is indicated by a pressure drop of more than 2 psig in 24-hours. SUMMA canisters passing the pressure test are connected to the cleaning system and are evacuated to $<0.05 \mathrm{~mm} \mathrm{Hg}$ for a minimum of one hour. The canisters are re-pressurized with humid zero air or nitrogen, and again evacuated. Canisters undergo a total of three evacuation/ pressurization cycles. At the end of the cleaning cycles, one canister per batch is pressurized with humid zero air or nitrogen and analyzed for contaminants.

Analysts must consult the most current revision of the QAPP for the criteria by which canisters are considered to be clean. 


\section{PROCEDURE 210.1}

\section{SUMMA` PASSIVATED STAINLESS STEEL CANISTER CERTIFICATION AND CLEANING}

\subsection{SCOPE AND APPLICATION}

1.1 This procedure describes how to clean and pressure certify SUMMA passivated stainless steel canisters. This procedure is based on the U.S. EPA Compendium Method TO-14, "The Determination of Volatile Organic Compounds (VOCs) in Ambient Air Using SUMMA Passivated Canister Sampling and Gas Chromatographic Analysis." SUMMA canisters used for the collection of headspace gases and VOCs will be certified for pressure and residual contamination. Tables listing the gas and VOC analytes are in the WIPP Experimental-Waste Characterization Program QAPP.

\subsection{SUMMARY OF PROCEDURE}

2.1 Canisters will be cleaned and certified on batch basis: A cleaning system, capable processing several canisters at a time, composed of an oven (optional) and a cryogenically trapped vacuum manifold will be used to clean the canisters. If an oil-free vacuum pump is used, the cryogenic trap is not necessary. Prior to cleaning, a 24-hour positive pressure leak test will be performed on all canisters. Any canister that fails, as indicated by a pressure drop of 2 psig or greater in 24 hours, will be checked for leaks and reprocessed. All canisters will be cleaned by repeated cycles of evacuation to $<0.05 \mathrm{mmHg}$ followed by pressurization with zero-air, nitrogen or helium. If the optional oven is used, a single evacuation cycle at $150^{\circ} \mathrm{C}$ for eight hours may be used. At the end of the cleaning cycle, all canisters used for collecting gases will be evacuated to $<0.05 \mathrm{mmHg}$ for storage. For canisters used to collect VOCs, one canister per batch will be filled with humid zero-air or nitrogen and analyzed for VOCs. The batch will be considered clean if there are no VOCs detected above $50 \%$ of the PROLs listed in Table 1.

\subsection{CANISTER CLEANING SYSTEM (see Figure 1)}

3.1 Complete canister cleaning system commercially available from Scientific Instruments Specialist, Moscow, ID. Model GS-ERS-A4.

3.2 Vacuum pump capable of evacuating sample canister(s) to an absolute pressure of $<0.05$ $\mathrm{mmHg}$. 
Table 1. VOC Headspace Target Compound List (TCL) and Program Required Quantitation Limits (PRQLs)

\begin{tabular}{|c|c|c|}
\hline Volatiles & CAS Number & PROL (ppmv) \\
\hline $\begin{array}{l}\text { 1. Acetone } \\
\text { 2. Benzene } \\
\text { 3. Bromoform } \\
\text { 4. 1-Butanol } \\
\text { 5. 2-Butanone }\end{array}$ & $\begin{array}{l}67-64-1 \\
71-43-2 \\
75-25-2 \\
71-36-3 \\
78-93-3\end{array}$ & $\begin{array}{c}100 \\
1 \\
1 \\
100 \\
100\end{array}$ \\
\hline $\begin{array}{l}\text { 6. Carbon Tetrachloride } \\
\text { 7. Chlorobenzene } \\
\text { 8. Chloroform } \\
\text { 9. Cyclohexane } \\
\text { 10. 1,1-Dichloroethane. }\end{array}$ & $\begin{array}{r}56-23-5 \\
108-90-7 \\
67-66-3 \\
110-82-7 \\
75-34-3\end{array}$ & $\begin{array}{l}1 \\
1 \\
1 \\
1 \\
1\end{array}$ \\
\hline $\begin{array}{l}\text { 11. 1,2-Dichloroethane } \\
\text { 12. 1,1-Dichloroethene } \\
\text { 13. cis-1,2-Dichloroethene } \\
\text { 14. Ethyl Benzene } \\
\text { 15. Ethyl Ether }\end{array}$ & $\begin{array}{r}107-06-2 \\
75-35-4 \\
156-59-2 \\
100-41-4 \\
60-29-7\end{array}$ & $\begin{array}{l}1 \\
1 \\
1 \\
1 \\
1\end{array}$ \\
\hline $\begin{array}{l}\text { 16. Methanol } \\
\text { 17. Methylene Chloride } \\
\text { 18. 4-Methyl-2-pentanone } \\
\text { 19. 1,1,2,2-Tetrachloroethane } \\
\text { 20. Tetrachloroethene }\end{array}$ & $\begin{array}{r}67-56-1 \\
75-09-2 \\
108-10-1 \\
79-34-5 \\
127-18-4\end{array}$ & $\begin{array}{c}100 \\
1 \\
100 \\
1 \\
1\end{array}$ \\
\hline $\begin{array}{l}\text { 21. Toluene } \\
\text { 22. 1,1,1-Trichloroethane } \\
\text { 23. Trichloroethene } \\
\text { 24. 1,1,2-Trichloro-1,2,2-trifluoroethane } \\
\text { 25. 1,3,5-Trimethylbenzene }\end{array}$ & $\begin{array}{r}108-88-3 \\
71-55-6 \\
79-01-6 \\
76-13-1 \\
108-67-8\end{array}$ & $\begin{array}{l}1 \\
1 \\
1 \\
1 \\
1\end{array}$ \\
\hline $\begin{array}{l}\text { 26. 1,2,4-Trimethylbenzene } \\
\text { 27. m-Xylene } \\
\text { 28. o-Xylene } \\
\text { 29. p-Xylene }\end{array}$ & $\begin{array}{r}95-63-6 \\
108-38-3 \\
95-47-6 \\
106-42-3\end{array}$ & $\begin{array}{l}1 \\
1 \\
1 \\
1\end{array}$ \\
\hline
\end{tabular}




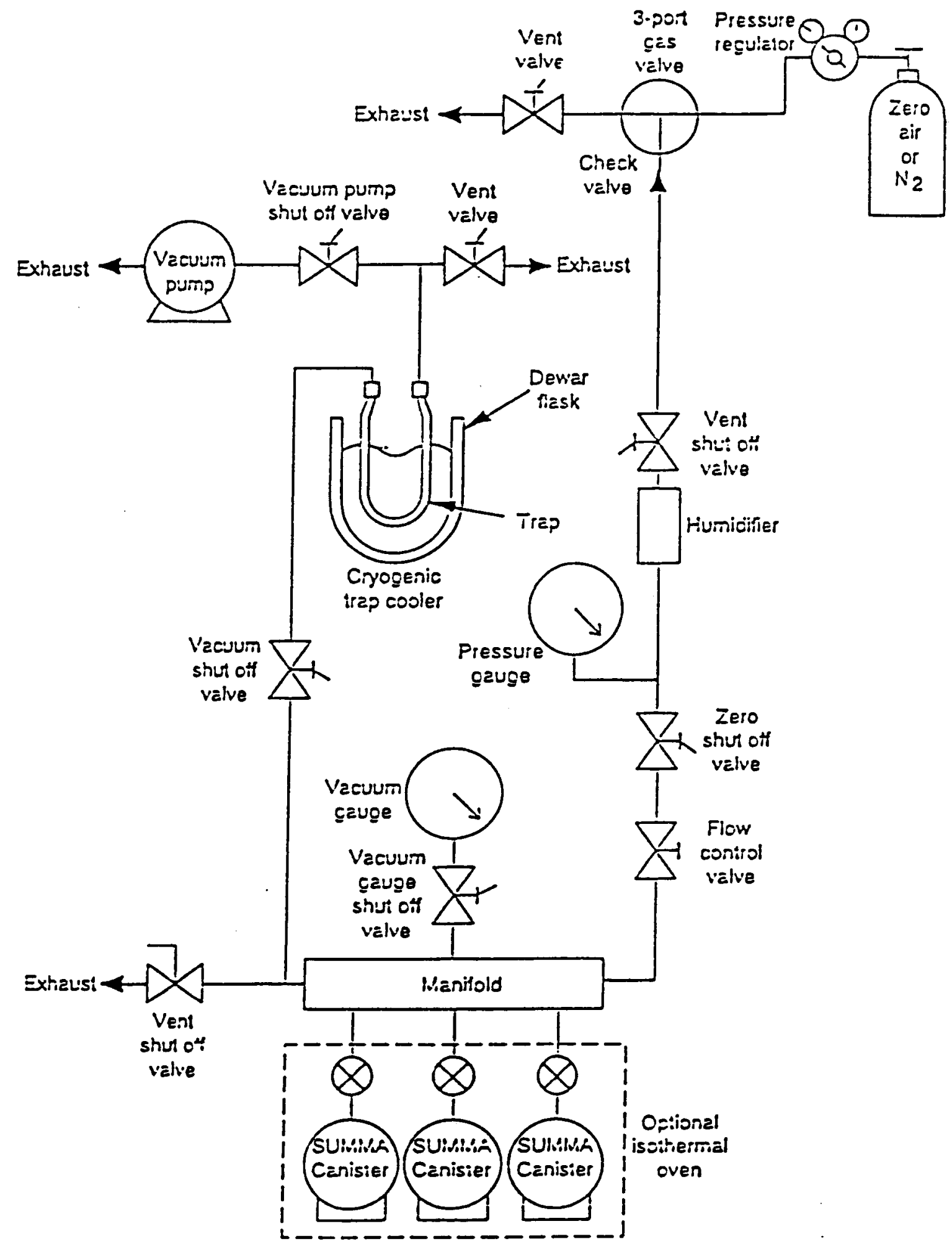

Figure 1. Canister Cleaning System 
3.3 Manifold constructed of stainless steel tubing with connections for simultaneously cleaning everal canisters.

3.4 Shut-off valves made of stainless steel and of the bellows type (Nupro " $\mathrm{H}^{\text {" }}$ series or equivalent).

3.5 Cryogenic trap made of stainless steel with an open tubular U-shaped trap cooled with liquid nitrogen to prevent contamination from back diffusion of oil from vacuum pump.

3.6 Humidifier - pressurizable water bubbler containing ASTM Type II water or equivalent, capable of providing moisture to the zero air or nitrogen supply.

3.7 Isothermal oven (optional) for heating canisters.

\subsection{CLEANING AND CERTIFICATION PROGRAM}

\subsection{Canister Cleaning and Certification}

4.1.1 All canisters used for VOCs must be clean and free $(<50 \%$ of PROLs) of any analytes listed in Table 1 prior to use.

4.1.2 All canisters are leak tested by pressurizing them to approximately 30 psig with zero air or nitrogen. The cleaning system in Figure 1 can be used for this task. The initial canister pressure is measured, the valve is closed and the final pressure is checked after 24 hours. The pressure must not vary by more than 2 psig over the 24-hour period.

4.1.3 A canister cleaning system may be assembled as shown in Figure 1 or purchased commercially (Scientific Instrument Specialist, Moscow, ID). The canisters are connected to the manifold. Cryogen is added to the trap. The vent shut-off valve and canister valve(s) are opened to release any remaining pressure in the canister(s). The vacuum pump is started and the shut-off valve is opened. If the optional oven is used, the temperature can be set to $150^{\circ} \mathrm{C}$. For canisters with integral vacuum/pressure gauges the maximum temperature is $125^{\circ} \mathrm{C}$ after removing the plastic face plate and rubber blow-out plug. The canisters are evacuated to $<0.05 \mathrm{mmHg}$ for a minimum of one hour. When the optional oven is used the canisters are evacuated for a minimum of eight hours. 
4.1.4 After one hour vacuum and vacuum/pressure gauge shut off valves are closed and the zero air/nitrogen shut-off valve is opened to pressurize the canisters with humid zero air or nitrogen to approximately 30 psig.

4.1.5 The zero air/nitrogen shut-off valve is closed and the canister(s) is allowed to vent down to atmospheric pressure through the vent shut-off valve. The vent shut-off valve is closed. Steps 4.1 .3 through 4.1 .5 are repeated two additional times for a total of three evacuation/ pressurization cycles for each set of canisters.

4.1.6 The optional oven is turned off, when canister(s) reach ambient temperature the vacuum and vacuum/pressure gauge shut-off valves are closed.

4.1.7 At the end of the evacuation/pressurization cycle, the canister or one canister from the set is pressurized to \pm 30 psig with humid zero air or nitrogen. The canister is then analyzed by the GCMS analytic system. If no compounds are detected at levels greater than $50 \%$ of the PROLs (Table 1), the canister(s) is clean. If contaminants are found at levels exceeding $50 \%$ of the PRQLs, then the cleaning procedure must be repeated until the canister(s) is clean.

4.1.8 The canister is reattached to the cleaning manifold and evacuated to $<0.05 \mathrm{mmHg}$ and remains in this condition until used. The canister valve is closed. The canister is removed from the cleaning system. 


\subsection{PHYSICAL WASTE FORM CHARACTERIZATION}

This Guidance Manual contains the following procedures for the physical waste form characterization of containerized wastes:

Procedure 310.1 provides a procedure for the non-destructive, $X$-ray scanning of waste containers using real-time radiography (RTR). Waste containers are placed in the path of $X$-rays which are then projected through the container and onto a fluorescent screen/image intensifier. The resulting image is transferred by a camera to a remotely located television screen. Containers are scanned while an operator monitors the television screen. Where applicable, RTR can be used to provide an estimate of the types and quantities of waste items, residual materials, and packaging materials and/or waste material categories. RTR can also be used to verify waste Content Code and site-specific Item Description Code, if applicable, and to estimate the quantity of free liquids, if present.

Procedure 310.2 provides a procedure for visually evaluating the type and amount of waste material in each container. Waste containers are opened, and their contents are visually examined by trained operators. Visual examination can be used to provide an estimate of the types and quantities of waste items, residual materials, and packaging materials and/or waste material categories. Visual examination can also be used to verify waste Content Code and site-specific Item Description Code, if applicable, and to estimate the quantity of free liquids, if present.

Analysts and operators must consult the most current revision of the QAPP for a discussion of applicable quality assurance objectives, calibration procedures and frequencies, and data reduction, validation, and reporting requirements. 
PROCEDURE 310.1

\section{REAL-TIME RADIOGRAPHY PROCEDURE}

\subsection{SCOPE AND APPLICATION}

1.1 Real-time radiography (RTR) is a non-destructive technique that involves $X$-ray scanning of waste containers. RTR is used by Department of Energy (DOE) generator/storage facilities to demonstrate compliance with the Waste Isolation Pilot Plant Waste Acceptance Criteria (Reference 5) and with the TRUPACT-II Authorized Methods for Payload Control (TRAMPAC) requirements (Reference 1). In the WIPP Experimental-Waste Characterization Program, RTR will be used in conjunction with visual examination to characterize waste for the WIPP bin-scale (Reference 4) and portions of the WIPP alcove tests. During the operational phase at the WIPP, it is expected that only RTR, not visual waste characterization, will be required. To achieve this end, the inventories of waste container contents obtained by RTR should be as detailed as possible.

1.2 In this procedure, RTR is used to evaluate the following: the waste inventory; the waste Content Code and/or Item Description Code (IDC), as appropriate; the waste packaging configuration; and the presence/absence and estimated volume of free liquids. This information is necessary for making weight estimates of waste items, residual materials, and packaging materials and/or waste material categories for well-segregrated waste.

\subsection{SUMMARY OF PROCEDURE}

2.1 RTR utilizes $X$-rays to inspect the contents of waste containers. This technique allows the operator to view events in progress (real timel, thus aiding in the location of free liquids by observing wave motion when the waste container is joggled.

2.2 The $\mathrm{X}$-ray producing device has controls which allow the operator to vary the voltage, thereby controlling the image quality. The imaging system typically utilizes a fluorescent screen and a low light television camera.

2.3 Waste containers are moved into the shielded vault, $X$-rays are projected through the container onto a fluorescent screen/image intensifier, and the resultant image is transferred by a camera to a remotely located television screen. A description of the contents of the drum is audio/video recorded and documented on a data form. The description should clearly identify all 
discernable waste items, residual and packaging materials, and/or waste material categories whenever possible so that waste can be classified according to the waste material categories specified in Table 1. This classification may involve the use of RTR information in conjunction with visual examination data during the bin-scale test phase.

\subsection{INTERFERENCES}

3.1 Containers of high density waste (e.g., leaded rubber, cemented sludges) can only be examined at their edges. In addition to this limitation, waste containers that are configured with a lead liner cannot be examined using RTR.

\subsection{APPARATUS AND MATERIALS}

4.1 A typical RTR system consists of the following equipment and facilities:

1. Shielded room that is properly ventilated and lighted

2. $X$-ray head and associated equipment

3. Drum turntable dolly assembly

4. Fluoroscopic screen and accessories

5. Closed-circuit television equipment and monitors

6. Safety interlocks

7. Data management system.

\subsection{REAGENTS}

Not applicable.

\subsection{SAMPLE COLLECTION, PRESERVATION, AND HANDLING}

Not applicable.

\subsection{PROCEDURE}

7.1 The exact sequence of operations is determined by the equipment available at individual RTR facilities. Waste containers selected for the WIPP bin-scale or alcove test (metal drums or boxes) are placed on a turntable/dolly and exposed to a collimated source of $X$-rays of sufficient intensity and beam geometry to permit a scan of container contents to be made for different orientations of the waste container. The contents of the waste container appear as a shadow image on a fluorescent 
Revision: 0

Date: June 14, 1991

Table 1. Waste Material Categories for Identification During RTR

\begin{tabular}{l} 
Cellulosics \\
Plastics \\
Rubber \\
Corroding metal/steel \\
Corroding metal/aluminum \\
Noncorroding metal \\
Solid inorganics \\
Inorganic sludges \\
Cements \\
Other organics \\
\hline \hline
\end{tabular}


screen that is in-line with the source of the $X$-rays and the waste container. The screen images resulting from different orientations of the waste container are recorded by videotaping the screen.

Drums are rotated to allow for total $X$-ray coverage, providing a quasi-three-dimensional image. However, boxes cannot be rotated during inspection, but are moved back and forth in front of the fluorescent screen to produce variation in the viewing angle to enhance waste identification. Boxes are first inspected from one side and then are rotated 180 degrees and are inspected from the other side.

During RTR, the waste container and its contents are examined, and a description of the container contents and packaging materials (e.g., container liner) is recorded according to written procedures. The description is detailed enough to determine whether the waste is composed of a single component (i.e., well-segregated/ homogeneous) and to determine whether the Content Code and/or IDC, as appropriate, has been properly assigned to the container. During this part of the examination, specify the height and shape of the waste in the container so that the volume utilization percentage can be determined.

Next, joggle the container by starting and stopping the turntable/dolly. After joggling the container, look for wave motion, a sign that liquids are present. Describe the location, container, and estimated volume of any liquids detected.

Finally, every effort should be made to provide an inventory of the waste container contents. This inventory should include a description of identifiable waste items, residual materials, and packaging materials and/or waste material categories. When possible, count the number of identical waste items present and estimate the amount of residual and packaging materials. Record this description of the waste container inventory and specific waste container identification data on the audio/video tape and on the RTR data form. It is understood that the amount of inventory information obtained by RTR will be limited when a heterogeneous waste form is examined. However, if the RTR inventory is judged to be comprehensive for a given drum, then the extent of the visual examination may be limited, and risk from exposure minimized.

\subsection{CALCULATIONS}

8.1 The identities of the waste items, residual and packaging materials and/or waste material categories documented on the audio/video tape and on the RTR data form are used in combination with a list of standard waste material weights (e.g., Table 2) to estimate the waste item, residual and packaging materials' and/or waste material categories' weights. Sites should develop a source of standard weights (an expansion of Table 2) based on knowledge of the waste and the process that generated it. Manufacturer's data or information from visual examination may also be used for this 
Table 2. Example Weights of Package Materials*

\begin{tabular}{lc}
\hline Item & Weight $^{\dagger}(\mathrm{kg})$ \\
\hline $210 \mathrm{~L}$ drum $(17 \mathrm{C})$ & 28 \\
90 mil liner $(210 \mathrm{~L})$ & 7.3 \\
Drum plastic bag $(210 \mathrm{~L})$ & 1.8 \\
Box fiberboard liner $(122 \times 122 \times 213 \mathrm{~cm})$ & 12.7 \\
Box plastic liner $(122 \times 122 \times 213 \mathrm{~cm})$ & 12 \\
Lead Brick $(5.1 \times 10 \times 20 \mathrm{~cm})^{*}$ & 12 \\
Lead Lined Drum: & 46 \\
$(0.32 \mathrm{~cm}$ thick, $71 \mathrm{~cm}$ high by $180 \mathrm{~cm}$ long) & 23 \\
$(0.16 \mathrm{~cm}$ thick, $71 \mathrm{~cm}$ high by $180 \mathrm{~cm}$ long $)$ & \\
\hline \hline
\end{tabular}

* This list is not intended to be comprehensive. Sites are expected to develop their own table based on knowledge of the wastes and the processes that generate them.

'Rocky Flats Plant

${ }^{t} p \mathrm{~Pb}=11.34 \mathrm{~g} / \mathrm{cm}^{3}$ 
purpose. It is recognized that visual examination data may be needed to ensure that waste material categories have been properly assigned.

The inventory and description of waste items, residual and packaging materials are used to classify the waste into waste material categories and to estimate the total weight of waste in each of these categories.

8.2 The utilized waste container volume percentage is estimated based on the highest point and shape of waste in a waste container. Figure 1 illustrates a typical waste drum and associated utilization volume percentage according to its height (assuming a flat top) within the drum. Individual sites must qualify when these percent values will not hold due to the presence of certain packaging materials (e.g., presence of fiber packs).

8.3 Table 3 is provided as a reference to aid in determining liquid quantities. Table 4 provides a list of RTR lens magnifications to aid in determining the physical size of waste items.

\subsection{QUALITY CONTROL}

9.1 Quality control during RTR operations is assured by operator training, equipment standards, replicate scans, and subsequent visual examination of the waste to verify RTR results.

9.2 Operator training and experience are the most important considerations for assuring the quality of the RTR data and for interpretation and disposition of RTR results. Only trained personnel are allowed to operate RTR equipment. Standardized training requirements for RTR operators are based upon existing industry standard training requirements and comply with the training and qualification requirements of ASME NOA-1, Element 2, except for Supplement 2S-2. Requalification of operators is based upon evidence of continued satisfactory performance (primarily tape reviews) and is done at least every two years. Unsatisfactory performance results in disqualification. Retraining and demonstration of satisfactory performance are required before an operator is again qualified to operate the RTR system. It is the responsibility of each generator/storage facility to determine the criteria by which an operator is considered qualified.

9.3 The RTR equipment is tuned precisely enough to allow an RTR operator to resolve a 2-2T hole in a steel block. The quality of the RTR image is also assessed by viewing an image test pattern. 
Revision: 0

Date: June 14, 1991

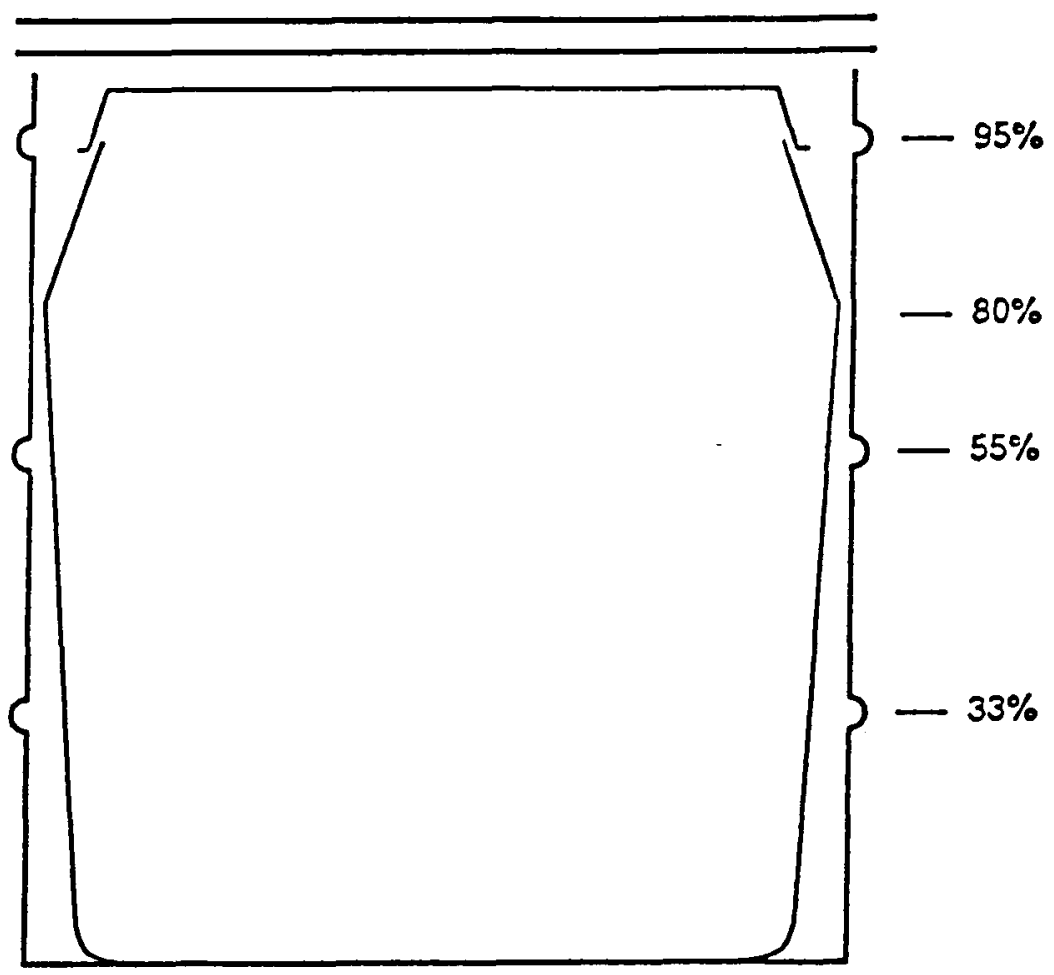

Volume utilization percentage to be based on the highest point of waste in drum* - From INEL SWEPP Sampling Program Plan

Figure 1. Volume Utilization Percentage 
Table 3. Volume Conversions

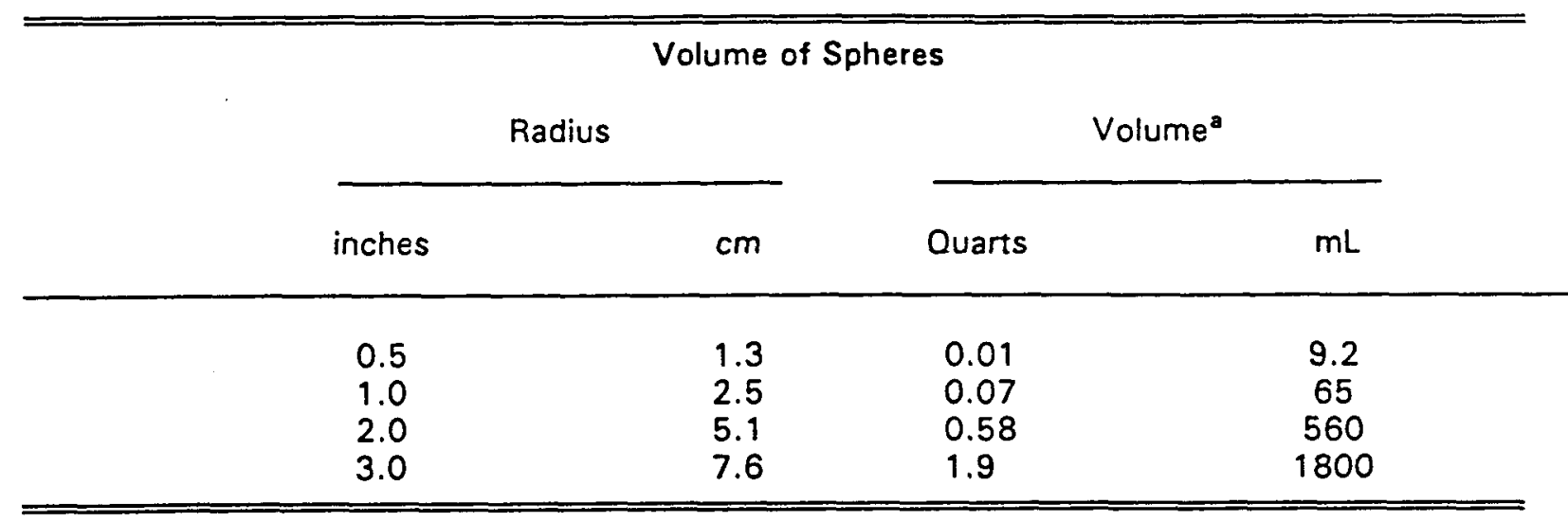

a Volume $=4 / 3 \pi r^{3}$

Volume of Half Spheres

\begin{tabular}{|c|c|c|c|}
\hline \multicolumn{2}{|c|}{ Radius } & \multicolumn{2}{|c|}{ Volume $^{b}$} \\
\hline inches & $\mathrm{cm}$ & Quarts & $\mathrm{mL}$ \\
\hline $\begin{array}{l}0.5 \\
1.0 \\
2.0 \\
3.0 \\
4.0\end{array}$ & $\begin{array}{l}1.3 \\
2.5 \\
5.1 \\
7.6 \\
10\end{array}$ & $\begin{array}{l}0.005 \\
0.035 \\
0.29 \\
0.95 \\
2.3\end{array}$ & $\begin{array}{c}4.6 \\
32.5 \\
280 \\
900 \\
2200\end{array}$ \\
\hline
\end{tabular}

bolume $=2 / 3 \pi r^{3}$

Volume of Cylinders ${ }^{c}$

Radius (inches/cm)

\begin{tabular}{ccccc}
\cline { 2 - 5 } $\begin{array}{c}\text { Height } \\
\text { (inches/cm) }\end{array}$ & $0.5 / 1.3$ & $1.0 / 2.5$ & $2.0 / 5.1$ & $3.0 / 7.6$ \\
\hline $0.50 / 1.3$ & $0.007 / 6.9$ & $0.01 / 9.8$ & $0.11 / 110$ & $0.25 / 240$ \\
$1.0 / 2.5$ & $0.014 / 13$ & $0.052 / 49$ & $0.22 / 200$ & $0.48 / 450$ \\
$2.0 / 5.1$ & $0.029 / 27$ & $0.11 / 100$ & $0.44 / 420$ & $0.98 / 930$ \\
$3.0 / 7.6$ & $0.043 / 40$ & $0.16 / 150$ & $0.66 / 620$ & $1.5 / 1400$ \\
$4.0 / 10$ & $0.056 / 53$ & $0.21 / 200$ & $0.86 / 820$ & $1.9 / 1800$ \\
\hline \hline
\end{tabular}

c Volume $=\pi r^{2} h$ (quarts $/ \mathrm{mL}$ ) 
Table 4. Typical Lens Magnification Factors*

\begin{tabular}{|c|c|c|c|}
\hline Lens $(\mathrm{mm})$ & Near Side & Middle & Far Side \\
\hline \multicolumn{4}{|c|}{ 55-Gallon Drums } \\
\hline 24 & 0.4 & 0.5 & 0.6 \\
\hline 35 & 0.5 & 0.65 & 0.8 \\
\hline 50 & 0.8 & 1.0 & 1.2 \\
\hline 60 & 1.2 & 1.45 & 1.7 \\
\hline 85 & 1.5 & 1.9 & 2.3 \\
\hline \multicolumn{4}{|c|}{ Boxes } \\
\hline 24 & 0.4 & 0.6 & 1.8 \\
\hline 35 & 0.6 & 0.9 & 2.5 \\
\hline 50 & 0.9 & 1.3 & 3.5 \\
\hline 60 & 1.3 & 2.2 & 6.2 \\
\hline 85 & 1.65 & 2.5 & 7.0 \\
\hline
\end{tabular}

*From INEL SWEPP Sampling Program Plan. 
9.4 Independent replicate scans are performed on one out of every 20 drums. In addition, supervisory personnel (also trained and qualified to the same standards) perform periodic tape reviews of accepted waste containers.

9.5 During the bin-scale test, RTR results are verified by visual examination (Procedure 310.2 ).

\section{REFERENCES}

1. "TRUPACT-11 Authorized Methods for Payload Control," Appendix 1.3.7, in Safety Analysis Report for the TRUPACT-Il Shipping Package, Revision 4, Nuclear Packaging, Inc., Federal Way, Washington, August 1989.

2. "Waste Characterization Program Plan for WIPP Experimental Waste," Revision O, January 1991.

3. Lappin, A.R., C.A. Gotway, M.A. Molecke, and R.L. Hunter, 1991, "Rationale for Revised WIPP Bin-Scale Gas-Generation Tests with CH TRU Wastes," SAND90-2481, Sandia National Laboratories, Albuquerque, New Mexico (in preparation).

4. Molecke, M.A., 1990, "Test Plan: WIPP Bin-Scale CH TRU Waste Tests," SAND90-1974, Sandia National Laboratories, Albuquerque, New Mexico.

5. USDOE, 1989, "TRU Waste Acceptance Criteria for the Waste Isolation Pilot Plant", DOEMIPP069, Rev. 3, Waste Isolation Pilot Plant, Carlsbad, New Mexico.. 
PROCEDURE 310.2

VISUAL EXAMINATION PROCEDURE

\subsection{SCOPE AND APPLICATION}

1.1 This procedure for the visual examination of the contents of a waste container is designed to provide data on the type and amount of material in each of the waste containers included in the binscale and a portion of the waste containers included in the WIPP alcove test. At the end of the binscale and alcove tests, the data will be evaluated to determine if, and to what extent, visual waste examination will be required for future WIPP operations. The bin-scale and alcove tests have been designed to investigate the rates at which $\mathrm{CH}$ TRU wastes generate gases under various simulated repository conditions.

1.2 The waste material categories that control gas generation rates are listed in Table 1. The estimated or measured weight of waste constituents within these ten waste material categories are determined using this procedure and will be used as input to a mathematical gas generation model.

\subsection{SUMMARY OF PROCEDURE}

2.1 The CH TRU waste that is designated for the WIPP bin-scale and a representative portion of waste for the WIPP alcove tests is visually examined, weights determined or estimated, and video recorded. A description and estimated or measured weights of the contents of each waste container is also recorded on audio/video tape and a visual examination data form. The description should clearly identify all discernible waste items, residual materials, packaging materials, or waste material categories so that all the waste in each container can be classified according to the waste material categories listed in Table 1. Technical experts who are experienced and trained waste operations personnel are expected to assess the need to open individual bags or packages of waste. Opening individual bags/packages may be necessary in order to ensure the quality of the examination data. If individual bags/packages are not opened, estimated weights of waste items, residual materials, packaging materials, or waste material categories within the bags/packages are recorded. If it is necessary to open individual bags/packages, then actual weights of waste items, residual materials, packaging materials, or waste material categories are recorded. Figure 1 illustrates the overall programmatic approach to visual characterization of the waste. 
Table 1. Waste Material Categories for Quantification

During Visual Examination and Example Waste

Materials in Each Category ${ }^{\dagger}$

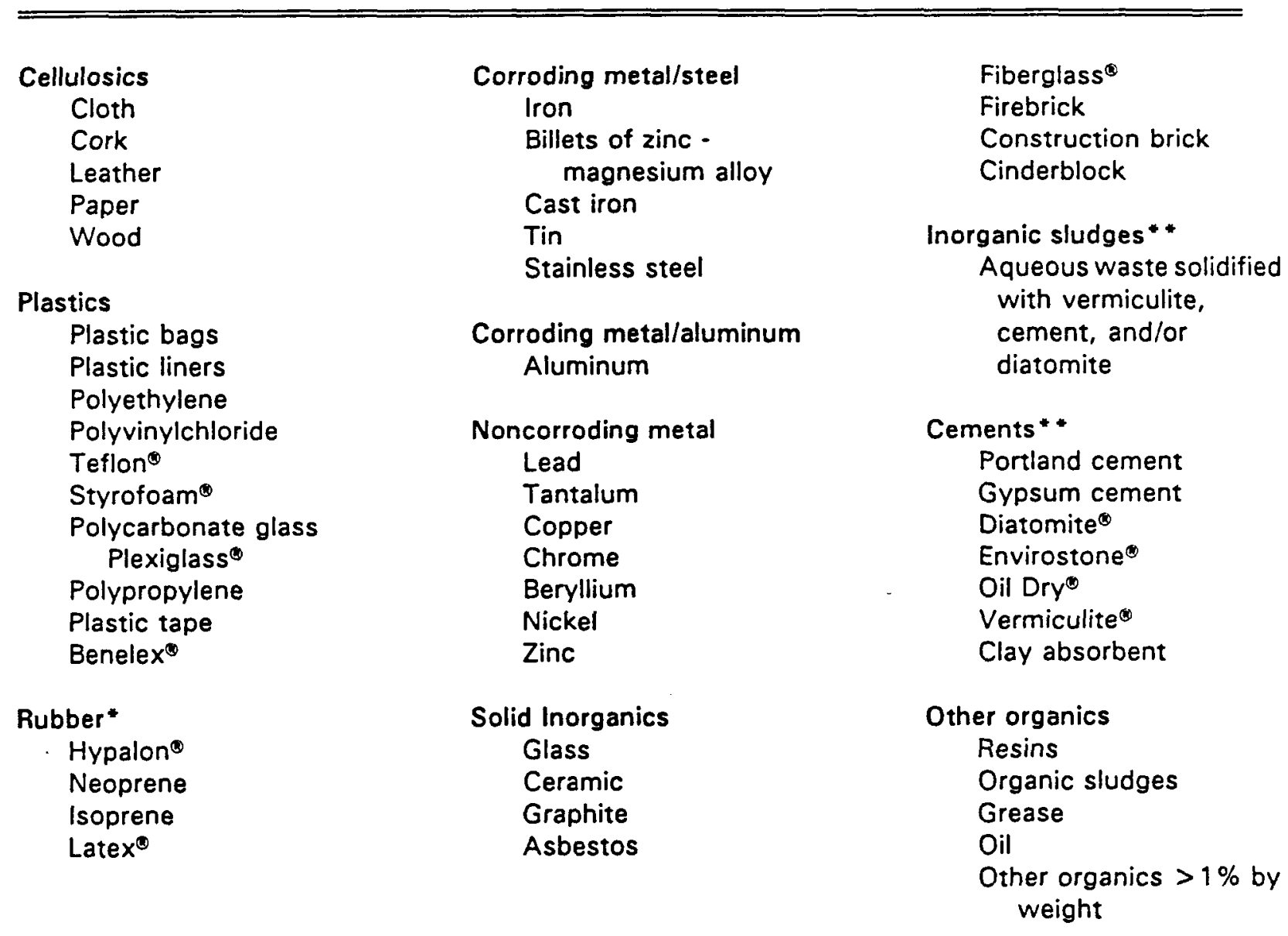

tAs much discrimination as is reasonable should be attempted within each waste material category.

*The amount of lead in leaded rubber should be determined using manufacturers' information. This approach should also be applied to other composite materials.

* An estimate of the weight percentage of sorbed water or other liquids, based on process knowledge, should be included with the description of materials in these categories. 


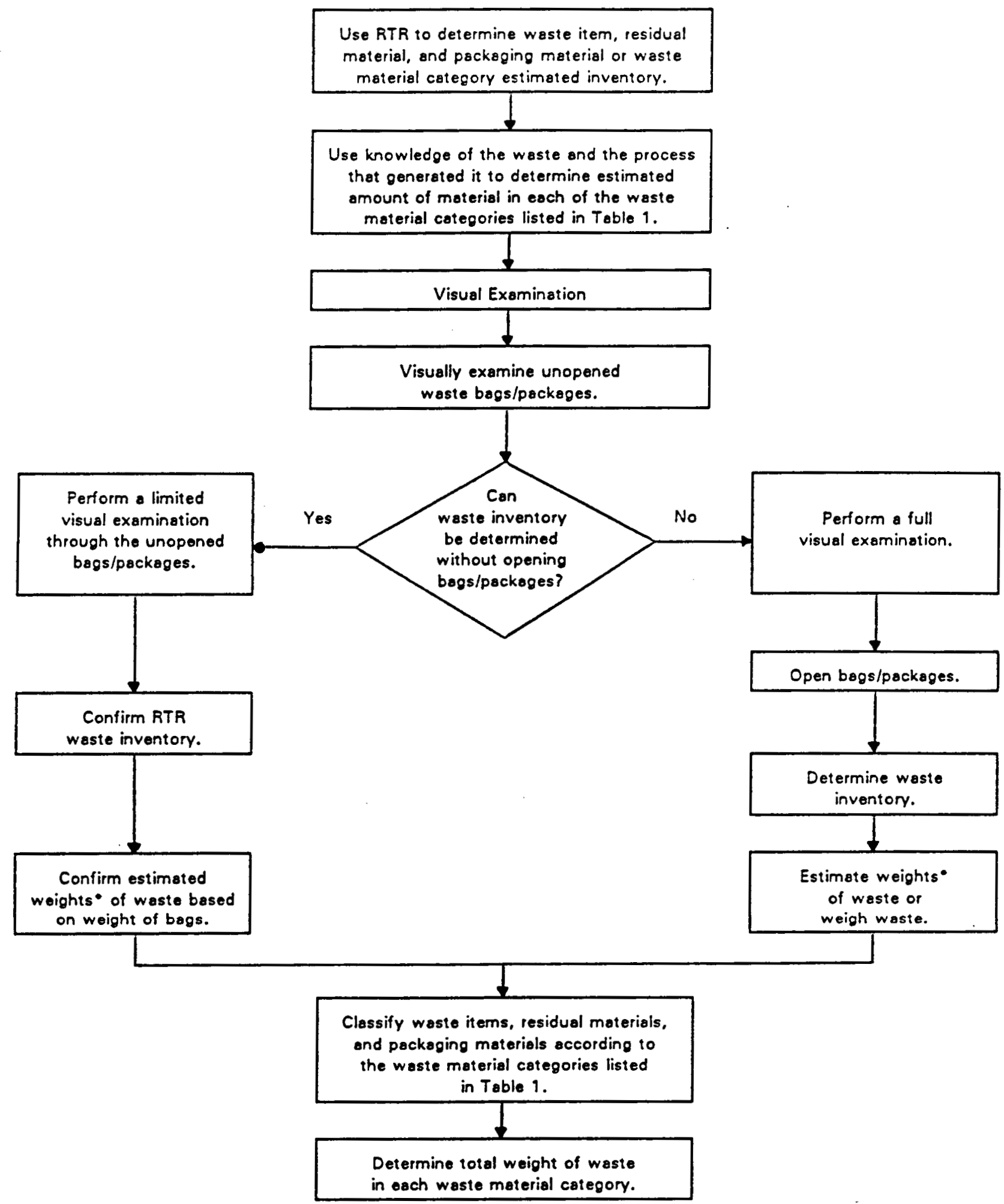

-Include process knowledge estimates of sorbed liquids, lead in leaded rubber, etc. for composite materials.

Figure 1. Overall Programmatic Approach to Visual Characterization of the Waste 


\subsection{INTERFERENCES}

3.1 It may not be possible to see through the inner plastic bags/packages of waste because of discoloration, grease and dust adhering to the interior surface of the bags/packages or because of sealed and taped containers within the bags/packages. In these cases, RTR data (Procedure 310.11), knowledge of the waste materials and the processes that generate them, and/or technical experts should assess the need to open the bags/packages in order to identify all of their contents. It should be understood that uncertainty in the total mass of waste in a waste material category leads to uncertainty in the gas generation model. Therefore, whether bags/packages are opened or not, it is important that the weight estimates be the best possible values. If they are not, additional testing may be required.

\subsection{APPARATUS AND MATERIALS}

4.1 The generator/storage sites develop their own radiation containment facilities and provide the following equipment:

- Drum, waste bag, and waste handling equipment,

- Video cameras and audio equipment,

- Mass balances and calibration standards,

- Bag opening unit,

- Data input station, and

- Safety equipment.

\subsection{REAGENTS}

Not applicable.

\subsection{SAMPLE COLLECTION, PRESERVATION, AND HANDLING}

Not applicable.

\subsection{PROCEDURE}

7.1 Before beginning the visual examination, review all documented data related to the waste container and its contents. If the available information is judged by a technical expert to be comprehensive, then the extent of the visual examination may be limited. In some cases, a decision to open all bags/packages in a waste container of a particular Content Code and/or IDC, as appropriate, may be made in advance. 
7.2 After this initial assessment, remove the lid from the waste container land the 90 mil rigid liner lid, if present) and estimate the waste container volume utilization percentage. Next, open the poly liner(s) bag(s) and document the number of liners and their type. Remove the individual inner bags/packages, if present, from the poly liner(s). Label and weigh each inner bag/package using properly calibrated mass balances. Taking care to ensure that the video camera is properly focused, begin the visual examination of an unopened inner bag/package while recording the operator's verbal description of the inner bag/package's inventory.

7.3 This inventory should include a description of all waste items, residual materials, packaging materials, or waste material categories contained in the inner bag/package. Estimated weights of the waste items, residual materials, packaging materials, or waste material categories should also be recorded, both on the audiotape and on the visual examination data form. Reference tables such as Table 1 and 2 should be developed to assist the operator in making weight estimates and assigning waste to a particular waste material category. These tables should be updated and refined by DOE site personnel as the Program proceeds.

7.4 If the identity of waste items, residual materials, packaging materials, or waste material categories cannot be ascertained through the unopened bag/package, then technical experts decide if the inner bag/package must be opened and document their rationale. Technical experts also assess the accuracy of the waste Content Code and/or IDC documented on the RTR data form, as appropriate, and recommend changes, if necessary. Those inner bags/packages that require opening are examined and their contents sorted, weighed, and recorded. An inventory of loose waste items, residual materials, packaging materials, or waste material categories not contained in inner bags/packages is also recorded. This waste is also sorted and weighed. If liquids are encountered at any time during the visual examination, then a description of their location, container, and estimated volume is recorded.

7.5 Once the contents of the waste container have been removed and examined, the weight of the empty waste container and the weight of its rigid poly liner, if present, is determined and recorded. The gross weight of the waste container (container plus contents) is also transcribed from the RTR data form to the visual examination data form. The total number of bags/packages is also recorded on the data form. (Note: (1) All inner bags/packages must be punctured before bin loading (see Reference 3 for specific requirements); (2) The waste container rigid poly liner, if present, must not be placed in the bin.]

\subsection{CALCULATIONS}

8.1 The inventory of waste items, residual materials, packaging materials, or waste material categories is used in conjunction with knowledge of the wastes and the processes that generate them 
Table 2. Example Weights of Packaging Materials *

\begin{tabular}{lc}
\hline Item & Weight ${ }^{\dagger}(\mathrm{kg})$ \\
\hline $210 \mathrm{~L}$ drum $(17 \mathrm{C})$ & 28 \\
90 mil liner $(210 \mathrm{~L})$ & 7.3 \\
Drum plastic bag $(210 \mathrm{~L})$ & 1.8 \\
Box fiberboard liner $(122 \times 122 \times 213 \mathrm{~cm})$ & 12.7 \\
Box plastic liner $(122 \times 122 \times 213 \mathrm{~cm})$ & 12 \\
Lead Brick $(5.1 \times 10 \times 20 \mathrm{~cm})^{\ddagger}$ & 12 \\
Lead Lined Drum: ${ }^{*}$ & 46 \\
$(0.32 \mathrm{~cm}$ thick, $71 \mathrm{~cm}$ high by $180 \mathrm{~cm}$ long $)$ & 23 \\
$(0.16 \mathrm{~cm}$ thick, $71 \mathrm{~cm}$ high by $180 \mathrm{~cm}$ long $)$ & \\
\hline \hline
\end{tabular}

*This list is not intended to be comprehensive. Sites are expected to develop their own table based on knowledge of the wastes and the processes that generate them.

${ }^{\dagger}$ Rocky Flats Plant

${ }^{\ddagger} \rho \mathrm{Pb}=11.34 \mathrm{~g} / \mathrm{cm}^{3}$ 
to classify the waste container contents into the waste material categories listed in Table 1. As much discrimination as possible between materials in a particular category should be attempted. However, for purposes of the bin-scale tests, only the total weight $(\mathrm{kg})$ of material within each of the ten waste material categories is required.

8.2 Table 3 is provided as a reference to aid in determining liquid quantities by determining the volume of the containers in which they occur.

\subsection{QUALITY CONTROL}

9.1 A specific waste recognition training program for each site is used to assess operator performance before actual waste characterization by visual examination. This training program employs exercises such as evaluation of simulated waste (non-radioactive materials), review of audio/videotapes of previous waste handling operations, including RTR, and familiarization with estimating and weighing techniques. Standard nomenclature is established, based on current site practice, to assure that all operators recognize waste by the same descriptors. The goal of the training and evaluation program is to achieve the accuracy discussed in the Sandia Test Plan and Addendum (Molecke, 1990; Molecke and Lappin, 1990).

9.2 Standard error calculation procedures are developed with the concurrence of Sandia National Laboratories. Involved DOE sites should coordinate these efforts to assure maximum consistency.

9.3 Replicate weight measurements are performed and used as a measure of the precision of weighing process. One in twenty, or at least one waste item, residual and packaging material, or waste material category from each drum, is reweighed after all other drum contents are weighed.

9.4 The documented and audio/visual taped results of the RTR evaluation of a drum are available to visual examination personnel prior to, or during the visual examination.

9.5 Mass balances, spanning a range of weights from $10 \mathrm{~g}$ to $450 \mathrm{~kg}(1000 \mathrm{lbs})$, are checked prior to use and calibrated annually. The weighing system is calibrated using standards traceable to NIST. 
Revision: 0

Date: June 14, 1991

Table 3. Volume Conversions

\begin{tabular}{|c|c|c|c|}
\hline \multicolumn{4}{|c|}{ Volume of Spheres } \\
\hline \multicolumn{2}{|c|}{ Radius } & \multicolumn{2}{|c|}{ Volume ${ }^{a}$} \\
\hline inches & $\mathrm{cm}$ & Quarts & $\mathrm{mL}$ \\
\hline $\begin{array}{l}0.5 \\
1.0 \\
2.0 \\
3.0\end{array}$ & $\begin{array}{l}1.3 \\
2.5 \\
5.1 \\
7.6\end{array}$ & $\begin{array}{l}0.01 \\
0.07 \\
0.58 \\
1.9\end{array}$ & $\begin{array}{c}9.2 \\
65 \\
560 \\
1800\end{array}$ \\
\hline
\end{tabular}

a Volume $=4 / 3 n r^{3}$

Volume of Half Spheres

\begin{tabular}{|c|c|c|c|}
\hline \multicolumn{2}{|c|}{ Radius } & \multicolumn{2}{|c|}{ Volume $^{b}$} \\
\hline inches & $\mathrm{cm}$ & Quarts & $\mathrm{mL}$ \\
\hline $\begin{array}{l}0.5 \\
1.0 \\
2.0 \\
3.0 \\
4.0\end{array}$ & $\begin{array}{l}1.3 \\
2.5 \\
5.1 \\
7.6 \\
10\end{array}$ & $\begin{array}{l}0.005 \\
0.035 \\
0.29 \\
0.95 \\
2.3\end{array}$ & $\begin{array}{c}4.6 \\
32.5 \\
280 \\
900 \\
2200\end{array}$ \\
\hline
\end{tabular}

bolume $=2 / 3 \pi r^{3}$

Volume of Cylinders ${ }^{c}$

Radius (inches $/ \mathrm{cm}$ )

\begin{tabular}{ccccc}
\cline { 2 - 5 } $\begin{array}{c}\text { Height } \\
\text { (inches } / \mathrm{cm} \text { ) }\end{array}$ & $0.5 / 1.3$ & $1.0 / 2.5$ & $2.0 / 5.1$ & $3.0 / 7.6$ \\
\hline & $0.007 / 6.9$ & $0.01 / 9.8$ & $0.11 / 110$ & $0.25 / 240$ \\
$0.50 / 1.3$ & $0.014 / 13$ & $0.052 / 49$ & $0.22 / 200$ & $0.48 / 450$ \\
$1.0 / 2.5$ & $0.029 / 27$ & $0.11 / 100$ & $0.44 / 420$ & $0.98 / 930$ \\
$2.0 / 5.1$ & $0.043 / 40$ & $0.16 / 150$ & $0.66 / 620$ & $1.5 / 1400$ \\
$3.0 / 7.6$ & $0.056 / 53$ & $0.21 / 200$ & $0.86 / 820$ & $1.9 / 1800$ \\
$4.0 / 10$ & &
\end{tabular}

c Volume $=\pi r^{2} h$ (quarts $/ \mathrm{mL}$ ) 
9.6 The other equipment used during visual examination includes audio/video recording apparatus. Checks of this equipment include observation of test patterns, prior to each day's use, to ensure that the video picture and record meet minimum quality requirements.

9.7 Finally, two operators concur on the results of the visual examination by signing the data form.

\subsection{REFERENCES}

1. Lappin, A.R., C.A. Gotway, M.A. Molecke, R.L. Hunter, and E.N. Lorusso, 1991, "Rationale for Revised WIPP Bin-Scale Gas-Generation Tests with CH TRU Wastes," SAND90-2481. Sandia National Laboratories, Albuquerque, New Mexico.

2. Molecke, M.A., and A. R. Lappin, 1990, "Test Plan Addendum \#1: WIPP Bin-Scale CH TRU Waste Tests," SAND90-2082, Sandia National Laboratories, Albuquerque, New Mexico.

3. Molecke, M.A., 1990, "Test Plan: WIPP Bin-Scale CH TRU Waste Tests," SAND90-1974. Sandia National Laboratories, Albuquerque, New Mexico. 


\subsection{ORGANICS}

This Guidance Manual contains the following procedures for the analysis of organic compounds in waste container headspace:

Procedure 430.1 provides a GCMS procedure for the determination of volatile organic compounds (VOCs) in waste container headspace samples that have been collected using SUMMA ${ }^{\otimes}$ passivated canisters. SUMMA canisters containing samples are attached to a sample manifold and the sample is diluted. The canister containing the diluted sample is attached to the analytical system and the VOCs are concentrated by collection in a cryogenically cooled trap or in a solid sorbent trap. VOCs are then separated on a widebore capillary column housed in a gas chromatograph, and detected using a mass spectrometer operating in the full scan mode.

Procedure 430.2 provides a GCMS procedure for the determination of VOCs in waste container headspace samples that have been collected using SUMMA* passivated canisters. SUMMA ${ }^{\star}$ canisters containing samples are attached to a sample manifold and the sample is diluted. Using a gas-tight syringe, an appropriate aliquot is removed from the diluted canister and injected into a $25 \mathrm{~mL}$ sparge tube that contains $5 \mathrm{~mL}$ reagent water spiked with internal standards. A purge-and-trap device efficiently transfers the VOCs from the sparge vessel to the head of a widebore capillary column housed in a gas chromatograph. The VOCs are detected using a mass spectrometer operating in the full scan mode.

Procedure 440.1 provides a GC procedure for the determination of alcohols and ketones in waste container headspace samples that have been collected using SUMMA passivated canisters. SUMMA canisters containing samples are pressurized to a minimum of two times ambient atmospheric pressure, and then attached directly to heated gas injection valves via a heated transfer line. The positive pressure of the sample canister fills integral sample loops. The sample loop aliquots are then transferred to two different widebore capillary columns housed in a gas chromatograph, and the alcohols and ketones are detected with flame ionization detectors (FIDs).

Analysts must consult the most current revision of the QAPP for applicable quality assurance objectives, calibration procedures and frequencies, and data reduction, validation, and reporting requirements. 
PROCEDURE 430.1

\section{MODIFIED METHOD TO-14 FOR THE DETERMINATION OF VOLATILE ORGANIC COMPOUNDS IN WASTE CONTAINER HEADSPACE USING SUMMA PASSIVATED CANISTER SAMPLING AND GAS CHROMATOGRAPHIC/MASS SPECTROMETRIC ANALYSIS}

\subsection{SCOPE AND APPLICATION}

1.1 This document describes a procedure for the analysis of volatile organic compounds (VOCs) listed in Table 1 in the range from $1 \mathrm{ppmv}$ (ppm, volume/volume) to volume percent, that have been collected from waste container headspace using SUMMA passivated canisters. This procedure is based on the U.S. EPA Compendium Method TO-14, "The Determination of Volatile Organic Compounds (VOCs) in Ambient Air Using SUMMA ${ }^{\circledR}$ Passivated Canister Sampling and Gas Chromatographic Analysis," and modifications made to this procedure by the EPA Region IV laboratory (Stephenson). The procedures described in this document for the analysis of VOCs collected in SUMMA canisters restrict the analytical options to gas chromatography/mass spectrometry (GCMS). The sampling procedures are described elsewhere in the QAPP for the WIPP Experimental-Waste Characterization Program.

1.2 The modifications to Method TO-14 described in this procedure are designed to enable the analyst to have more flexibility when analyzing high concentrations of VOCs (>1 ppmv), and the ability to analyze polar compounds (alcohols and ketones). Modifications included in this procedure are those developed and implemented by the EPA Region IV laboratory (Stephenson). These modifications include using internal standards and solid sorbent traps. Solid sorbent trapping is achieved using commercially available purge-and-trap systems (Tekmar LSC-2 or equivalent). Solid sorbent traps containing only hydrophobic materials (activated carbon and carbon molecular sieves) allow the use of a dry purge cycle to remove trapped water from the trap prior to thermal desorption of trapped VOCs. Nafion dryers will not be used to remove water prior to the trapping of VOCs because of the possibility of removing polar compounds.

1.3 The procedures described herein are applicable to the VOCs specified in Table 1. The EPA has not determined the stability of alcohols and ketones when stored in pressurized or sub-ambient SUMMA canisters. It is anticipated that no adverse problems will be encountered with these types of compounds when stored in SUMMA* canisters due to the concentration levels at which these compounds are expected to be found. 
Table 1. VOC Headspace Target Compound List (TCL) and Program Required Quantitation Limits (PRQLs)

\begin{tabular}{|c|c|c|}
\hline Volatiles & CAS Number & PROL (ppmv) \\
\hline $\begin{array}{l}\text { 1. Acetone } \\
\text { 2. Benzene } \\
\text { 3. Bromoform } \\
\text { 4. 1-Butanol } \\
\text { 5. 2-Butanone }\end{array}$ & $\begin{array}{l}67-64-1 \\
71-43-2 \\
75-25-2 \\
71-36-3 \\
78-93-3\end{array}$ & $\begin{array}{c}100 \\
1 \\
1 \\
100 \\
100\end{array}$ \\
\hline $\begin{array}{l}\text { 6. Carbon Tetrachloride } \\
\text { 7. Chlorobenzene } \\
\text { 8. Chloroform } \\
\text { 9. Cyclohexane } \\
\text { 10. 1,1-Dichloroethane }\end{array}$ & $\begin{array}{r}56-23-5 \\
108-90-7 \\
67-66-3 \\
110-82-7 \\
75-34-3\end{array}$ & $\begin{array}{l}1 \\
1 \\
1 \\
1 \\
1\end{array}$ \\
\hline $\begin{array}{l}\text { 11. 1,2-Dichloroethane } \\
\text { 12. 1,1-Dichloroethene } \\
\text { 13. cis-1,2-Dichloroethene } \\
\text { 14. Ethyl Benzene } \\
\text { 15. Ethyl Ether }\end{array}$ & $\begin{array}{r}107-06-2 \\
75-35-4 \\
156-59-2 \\
100-41-4 \\
60-29-7\end{array}$ & $\begin{array}{l}1 \\
1 \\
1 \\
1 \\
1\end{array}$ \\
\hline $\begin{array}{l}\text { 16. Methanol } \\
\text { 17. Methylene Chloride } \\
\text { 18. 4-Methyl-2-pentanone } \\
\text { 19. 1,1,2,2-Tetrachloroethane } \\
\text { 20. Tetrachloroethene }\end{array}$ & $\begin{array}{r}67-56-1 \\
75-09-2 \\
108-10-1 \\
79-34-5 \\
127-18-4\end{array}$ & $\begin{array}{c}100 \\
1 \\
100 \\
1 \\
1\end{array}$ \\
\hline $\begin{array}{l}\text { 21. Toluene } \\
\text { 22. 1,1,1-Trichloroethane } \\
\text { 23. Trichloroethene } \\
\text { 24. 1,1,2-Trichloro-1,2,2-trifluoroethane } \\
\text { 25. 1,3,5-Trimethylbenzene }\end{array}$ & $\begin{array}{r}108-88-3 \\
71-55-6 \\
79-01-6 \\
76-13-1 \\
108-67-8\end{array}$ & $\begin{array}{l}1 \\
1 \\
1 \\
1 \\
1\end{array}$ \\
\hline $\begin{array}{l}\text { 26. 1,2,4-Trimethylbenzene } \\
\text { 27. m-Xylene } \\
\text { 28. o-Xylene } \\
\text { 29. p-Xylene }\end{array}$ & $\begin{array}{r}95-63-6 \\
108-38-3 \\
95-47-6 \\
106-42-3\end{array}$ & $\begin{array}{l}1 \\
1 \\
1 \\
1\end{array}$ \\
\hline
\end{tabular}


1.4 This procedure is based upon cryogenic or solid sorbent trapping followed by GCMS analysis and should be restricted to use by or under the supervision of analysts experienced in these areas. In addition, analysts should be skilled in the interpretation of mass spectra and their use as a quantitative tool.

1.5 The program required quantitation limits (PRQLs) specified in Table 1 are based on a 10 $\mathrm{mL}$ sample aliquot. PROLs will be proportionally higher for samples that require dilution or reduced sample size to avoid instrument saturation.

\subsection{SUMMARY OF PROCEDURE}

2.1 Upon receipt at the laboratory, a canister containing a sample is attached to the sample manifold (Figure 1). The sample is then diluted, either by direct pressurization or the transfer of an aliquot to an evacuated laboratory canister, and pressurized. The canister containing the diluted sample is attached to the analytical system (Figure 2). The VOCs are then concentrated by collection in a cryogenically cooled trap or in a solid sorbent trap. The temperature of the trap is raised and the VOCs are desorbed into the gas chromatograph. The VOCs are separated on a widebore $1 \geq 0.53 \mathrm{~mm}$ I.D.) capillary column and detected using a mass spectrometer operating in the full scan mode. The combination of GCMS allows for the qualitative and quantitative analysis of the VOCs contained in the SUMMA ${ }^{*}$ canister. The chromatographic conditions as well as typical mass spectrometer operating parameters are given in this procedure.

2.2 If a cryogenic trap is used, the trap is cooled with a cryogen and the gas sample is metered through the trap. The VOCs are condensed out and concentrated in the trap. The cryogen is then removed and the trap temperature is rapidly raised to revolatilize the retained VOCs directly onto the GC column. When a solid sorbent trap is used, the gas sample is metered through the solid sorbent where the VOCs are trapped. The solid sorbent trap is backflushed with inert gas to remove water and, after this dry purge cycle, the trap is heated and backflushed with inert gas to desorb the components onto the GC column.

\subsection{INTERFERENCES}

3.1 Contamination may occur in the analytical system if the canisters are not properly cleaned prior to use. Instructions for cleaning the canisters are described in Section 8.7. 


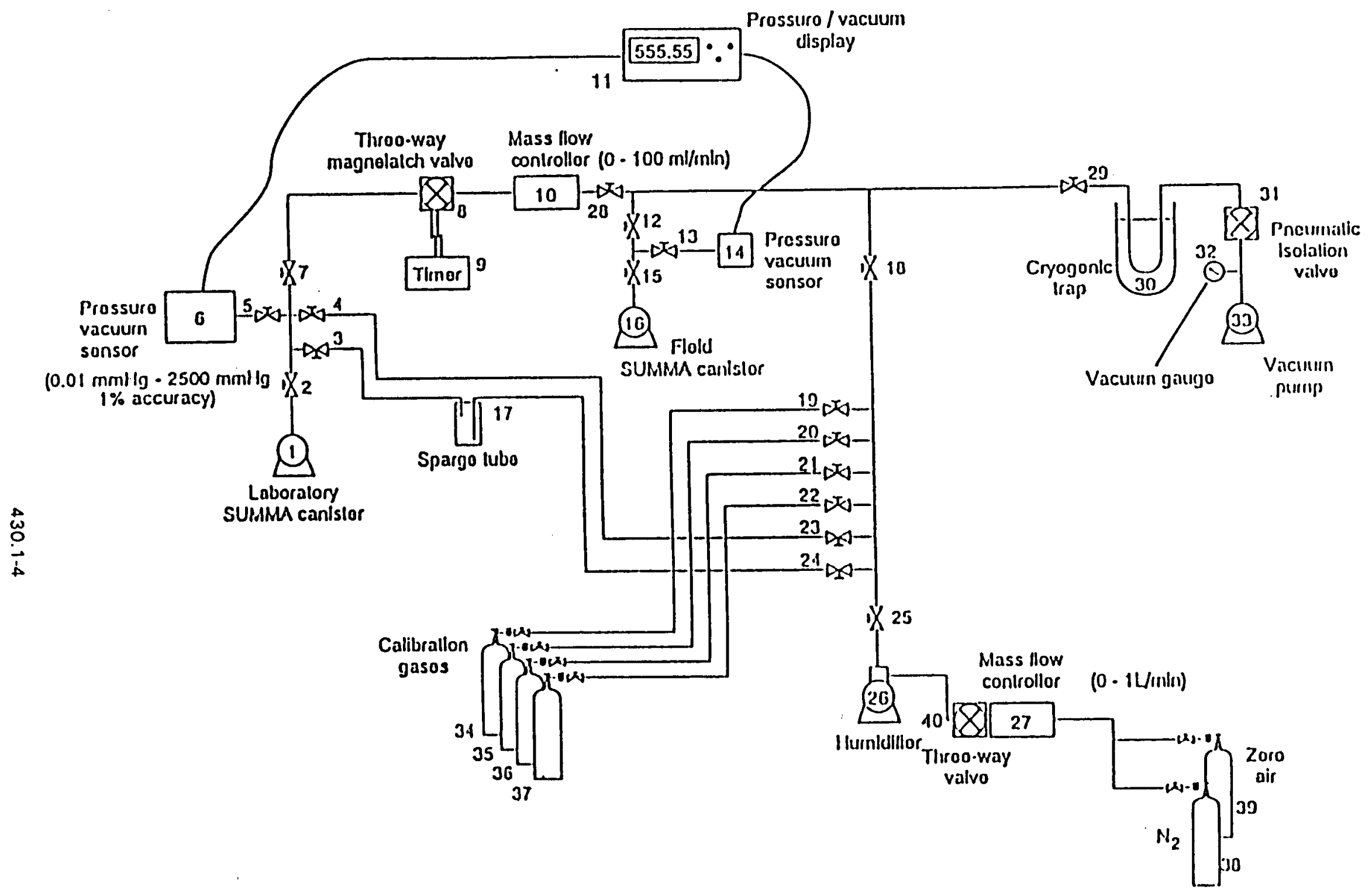

Figure 1. Sample and Calibration Standard Preparation Manifold 


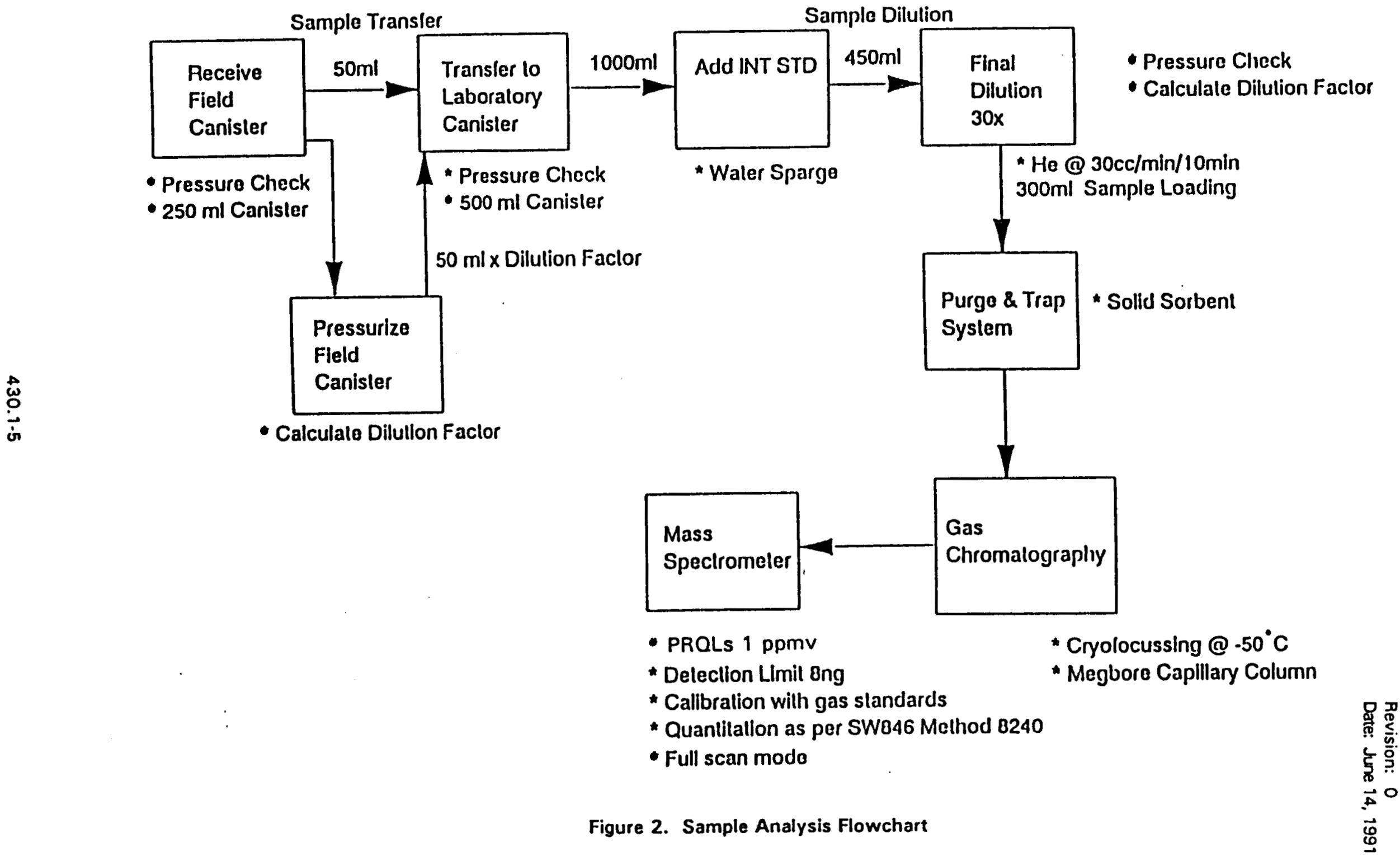


3.2 Cross-contamination can occur whenever high-level and low-level samples are analyzed sequentially. Whenever a high-level sample is analyzed, it should be followed by the analysis of ultra pure air or nitrogen.

3.3 The laboratory where VOC analysis is performed should be free of solvents.

3.4 In the analytical system, the use of non-Teflon plastic coating, non-Teflon thread sealants, or flow controllers with rubber components should be avoided.

\subsection{APPARATUS AND MATERIALS}

\subsection{Analytical System}

\subsubsection{Gas Chromatograph/Mass Spectrometer System}

\subsubsection{Gas chromatograph: Capable of sub-ambient oven temperature} programming. Optional capabilities for direct injection of liquid standards onto megabore columns. (Hewlett Packard, Model 5890 or equivalent).

\subsubsection{Column: 30 meter $\times 0.53 \mathrm{~mm}$ I.D. DB-624 (J \& W Scientific) or} equivalent.

4.1.1.3 Mass spectrometer: Capable of scanning from 29-260 amu every 3 seconds or less, using 70 volts (nominal) electron energy in the electron impact mode and producing a mass spectrum that meets all the criteria in Table 2 when $50 \mathrm{ng}$ of 4-bromofluorobenzene (BFB) are injected through the gas chromatograph inlet.

\subsubsection{GCMS interface: Any GC-to-MS interface that gives acceptable} calibration data at the PROLs specified in Table 1 for all analytes and achieves all acceptable instrument performance criteria (Table 2). Interfaces should be constructed of all glass materiais or glass-lined materials.

4.1.1.5 Data system: A computer system that allows the continuous acquisition and storage of all mass spectra obtained throughout the duration of the chromatographic program must be interfaced to the mass spectrometer. The computer must have software that allows the searching of a GCMS data file for ions of a specified mass and plotting such ion abundances versus time or scan 
Table 2. 4-Bromofluorobenzene Key lons and Abundance Criteria

\begin{tabular}{ll}
\hline Mass & \multicolumn{1}{c}{ lon Abundance Criteria } \\
\hline 50 & 15 to $40 \%$ of mass 95 \\
75 & 30 to $60 \%$ of mass 95 \\
95 & base peak, $100 \%$ relative abundance \\
96 & 5 to $9 \%$ of mass 95 \\
173 & less than $2 \%$ of mass 174 \\
174 & greater than $50 \%$ of mass 95 \\
175 & 5 to $9 \%$ of mass 174 \\
176 & greater than $95 \%$ but less than $101 \%$ of mass 174 \\
177 & 5 to $9 \%$ of mass 176 \\
\hline \hline
\end{tabular}


number. This type of plot is defined as an Extracted Ion Current Profile (EICP). Software must also be available that allows integrating the abundances in any EICP between specified time or scan-number limits. Data reduction software must be available that enables the qualitative and quantitative determination of target analytes using user-built mass spectral libraries and response factors based on EICP peak areas. The data system must be capable of forward library searching of the most recent NBS mass spectral library.

\subsubsection{Inlet/Trapping System (Figures 3 through 6)}

4.1.2.1 Cryogenic trap and desorber: Cryogenic trap with temperature control assembly (Nutech Corporation, Durham, NC, Model 320-01 or equivalent).

\subsubsection{Solid sorbent trap and desorber: This system is based on purge-and-trap} devices employed for the analysis of VOCs in aqueous samples. These devices are typically composed of a sample sparge vessel, a solid sorbent trap, and a desorber. Several complete systems are available commercially (Tekmar Company, Cincinnati, OH, Model LSC-2 with auto sampler or equivalent).

\subsection{Solid sorbent trap: The solid sorbent trap must be at least} $25 \mathrm{~cm}$ long and have an inside diameter of at least 0.105 inch. The recommended trap should have the following amounts of adsorbents (starting at the trap inlet): $7.6 \mathrm{~cm}$ of Carbopack B and $1.3 \mathrm{~cm}$ of Carbosieve S-III (Supelco, Supelco Park, Bellafonte, PA, Model 2-301 or equivalent). Before initial use, the trap should be conditioned for one hour at $260^{\circ} \mathrm{C}$ by backflushing with an inert gas flow of at least $30 \mathrm{~mL} / \mathrm{min}$. Vent the trap effluent to a hood, not to the analytical column. Prior to daily use, the trap should be conditioned for $10 \mathrm{~min}$ at $260^{\circ} \mathrm{C}$ with backflushing.

4.1 2.2.2 Desorber: The desorber should be capable of rapidly heating the trap to $220^{\circ} \mathrm{C}$ for desorption.

\subsubsection{Electronic mass flow controller: The electronic mass flow controller} must maintain constant flow for sample gas. The controller should be capable of indicating total flow (totalizer) through the controller (Matheson Model 8200-0422, 2-100 ml/min or equivalent).

\subsubsection{Vacuum pump: A diaphragm vacuum pump capable of drawing desired} sample volume through either the cryogenic or the solid sorbent trap (Scientific Instrument Specialists, Model NO5SV or equivalent). 


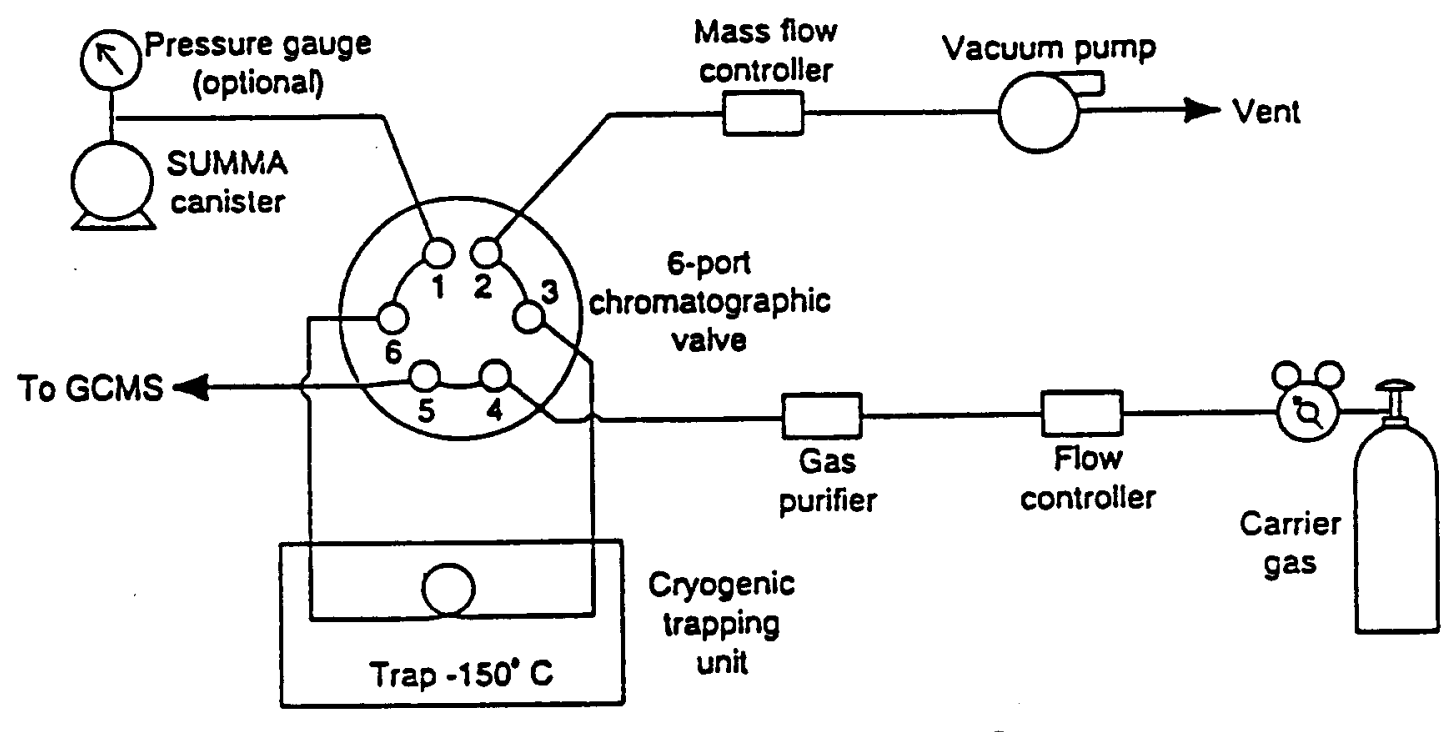

A. Sample trapping mode.

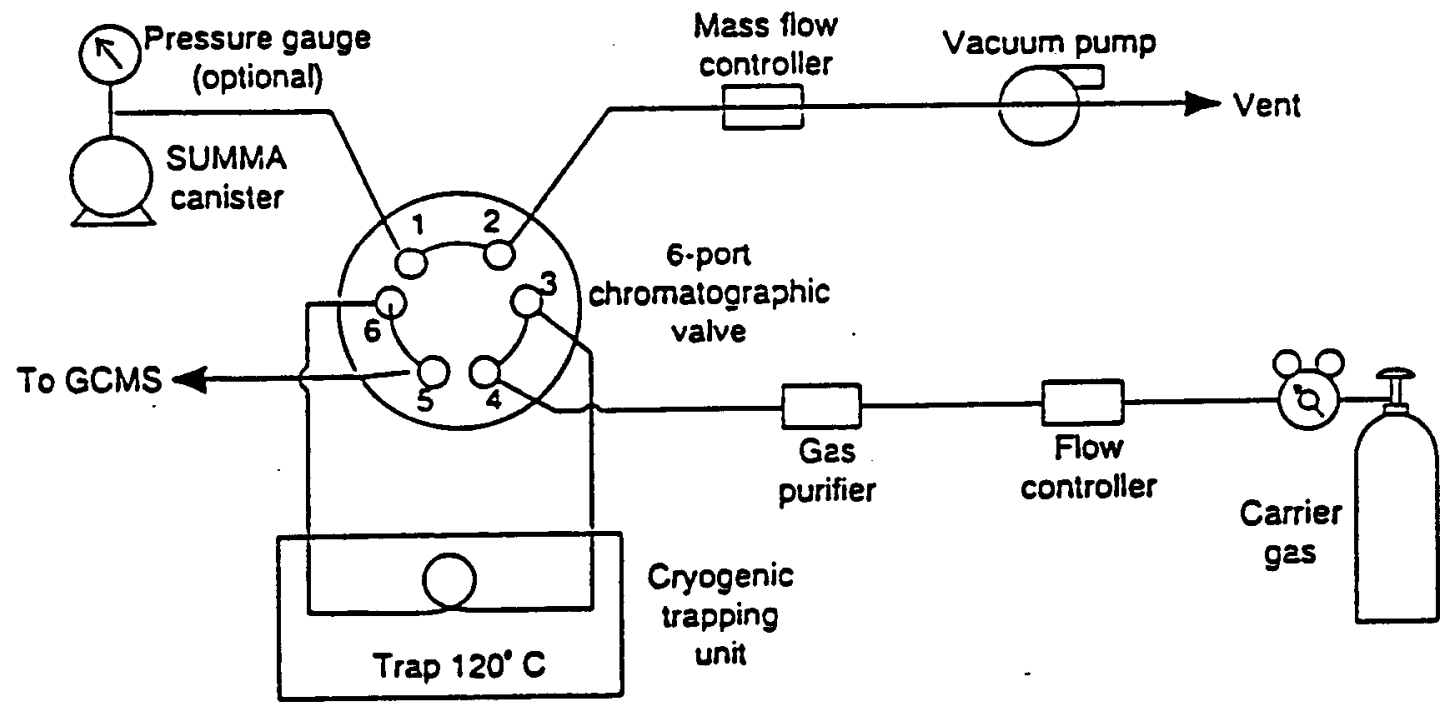

B. Sample desorption mode.

Figure 3. Cryogenic Trapping With Mass Flow Control 


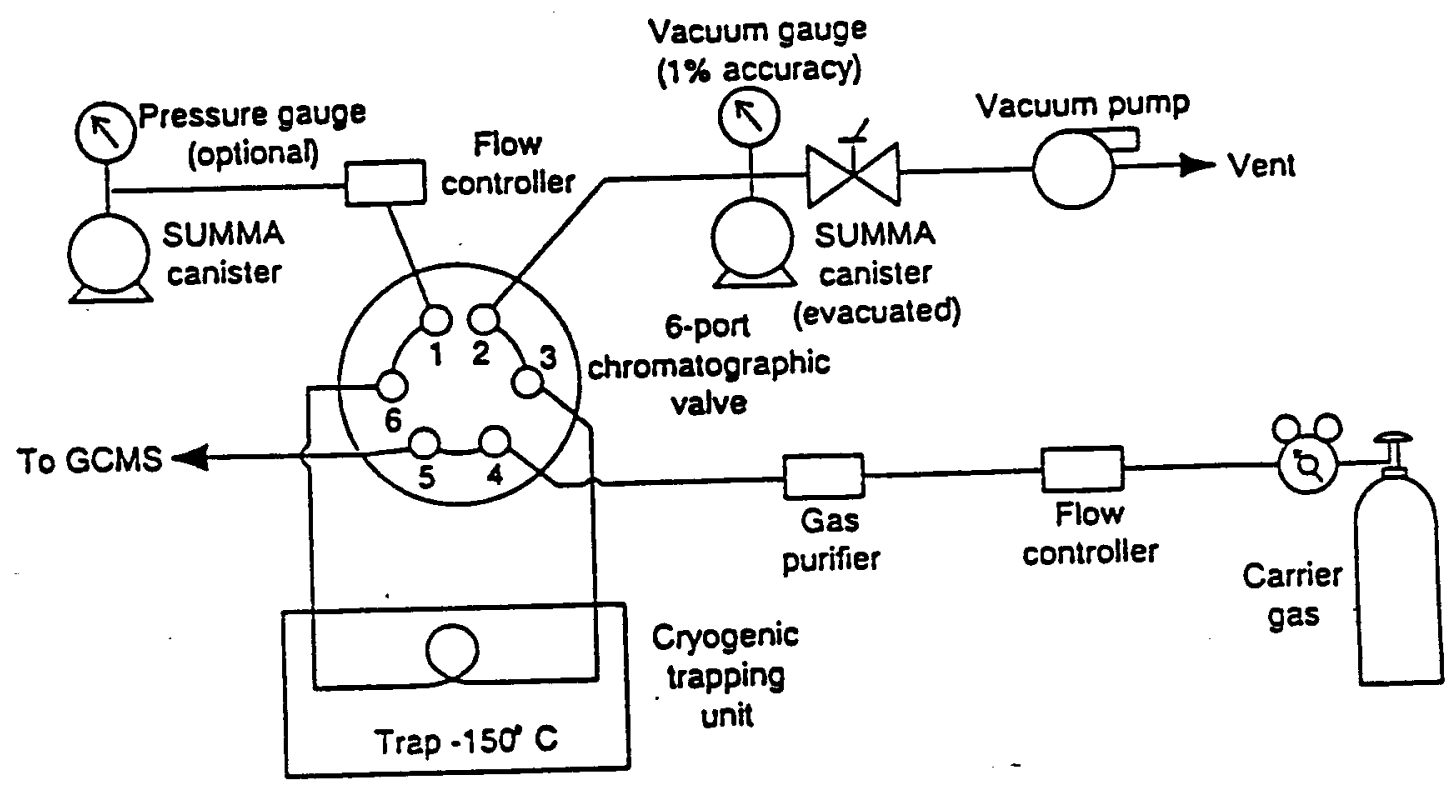

A. Sample trapping mode.

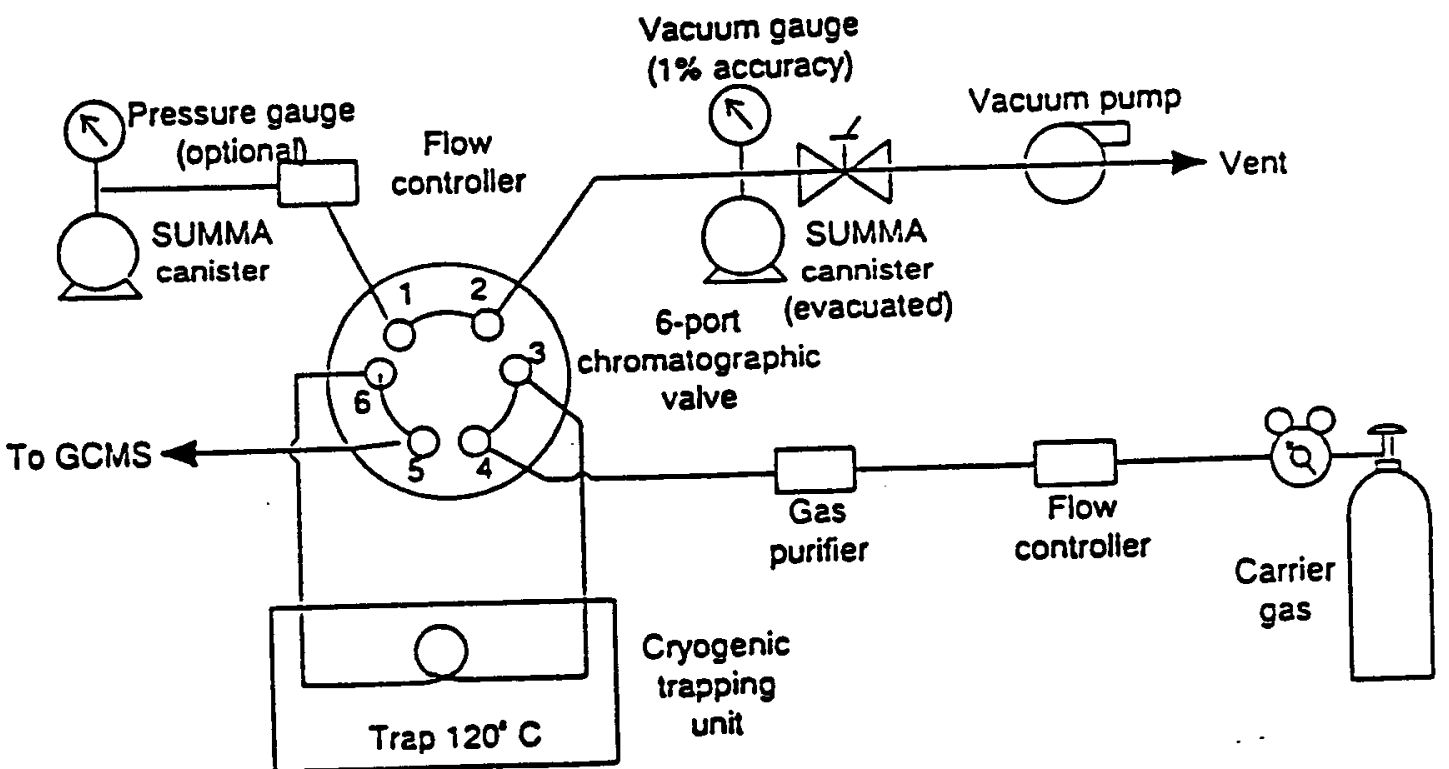

B. Sample desorption mode.

Figure 4. Cryogenic Trapping With Evacuated Canister 


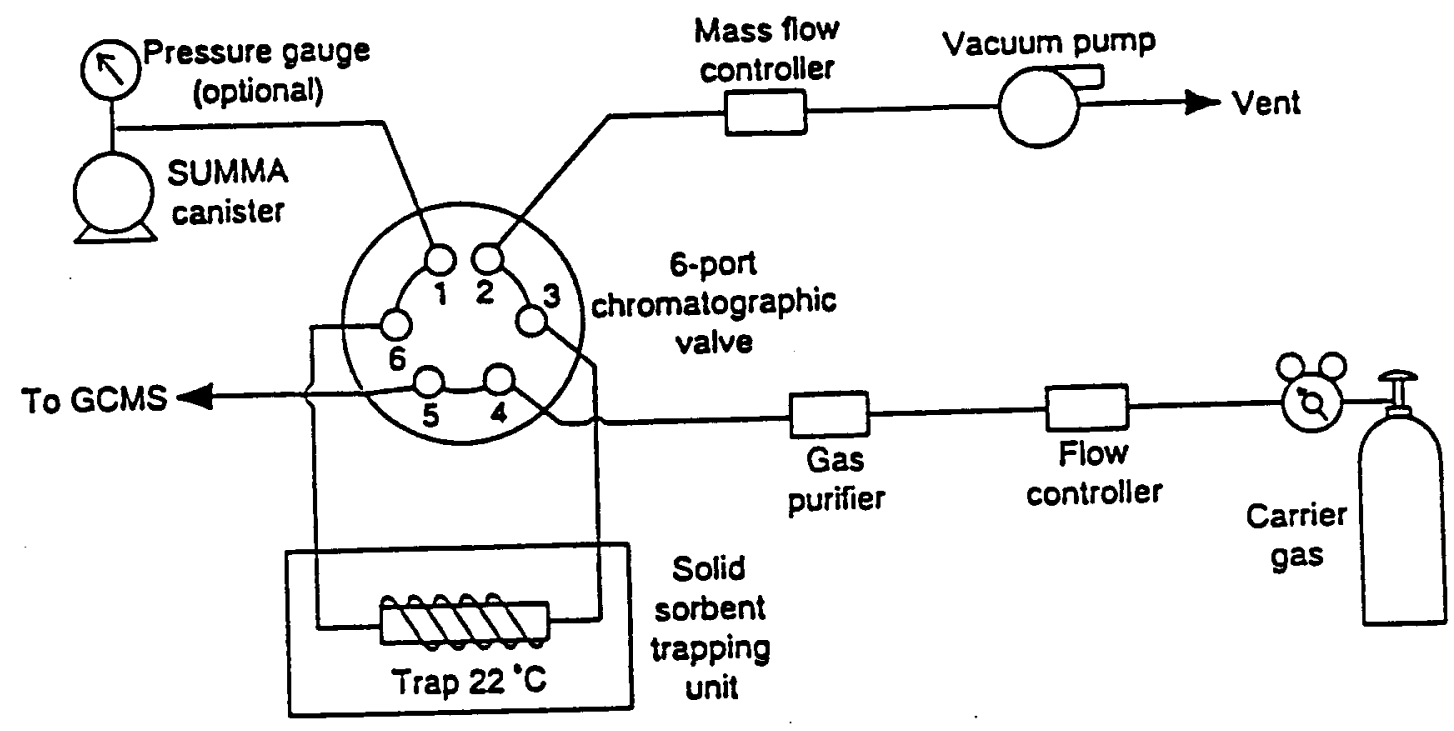

A. Sample trapping mode.

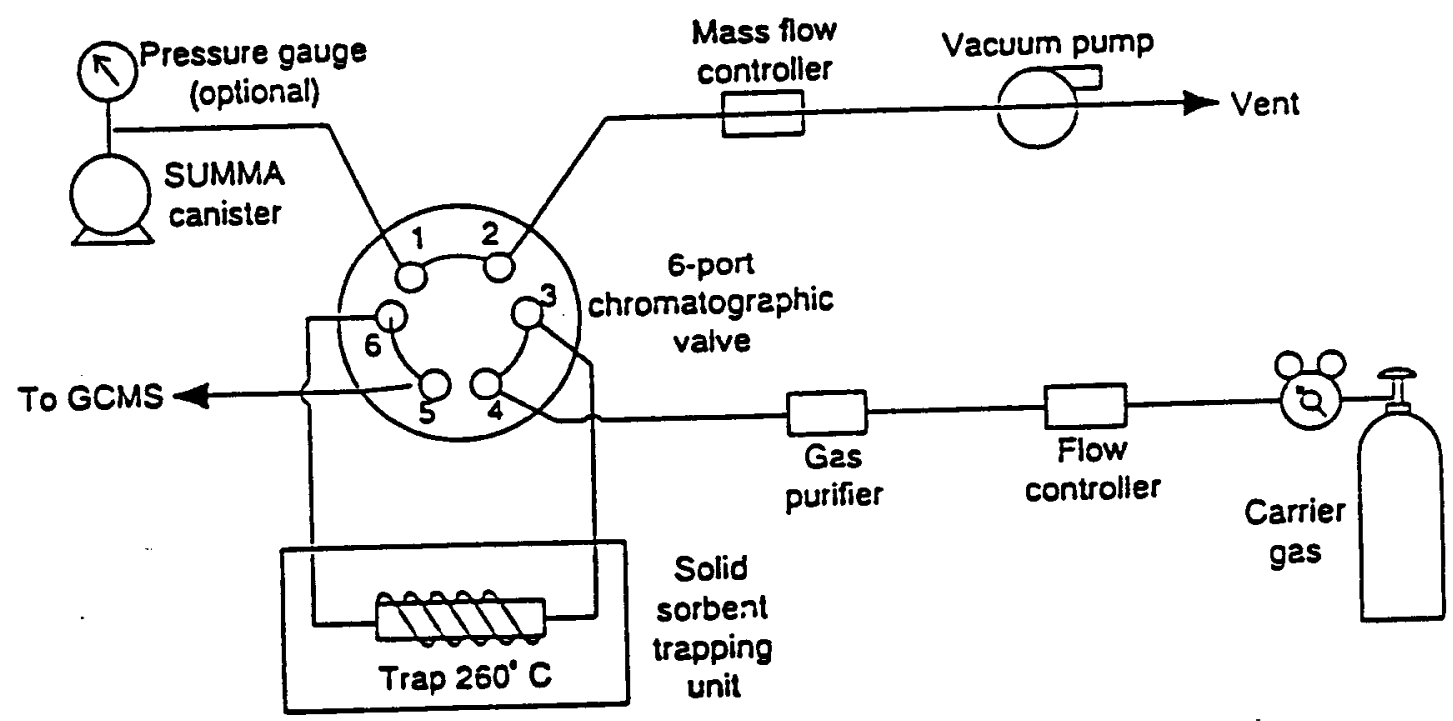

B. Sample desorption mode.

Figure 5. Solid Sorbent Trapping With Mass Flow Control 


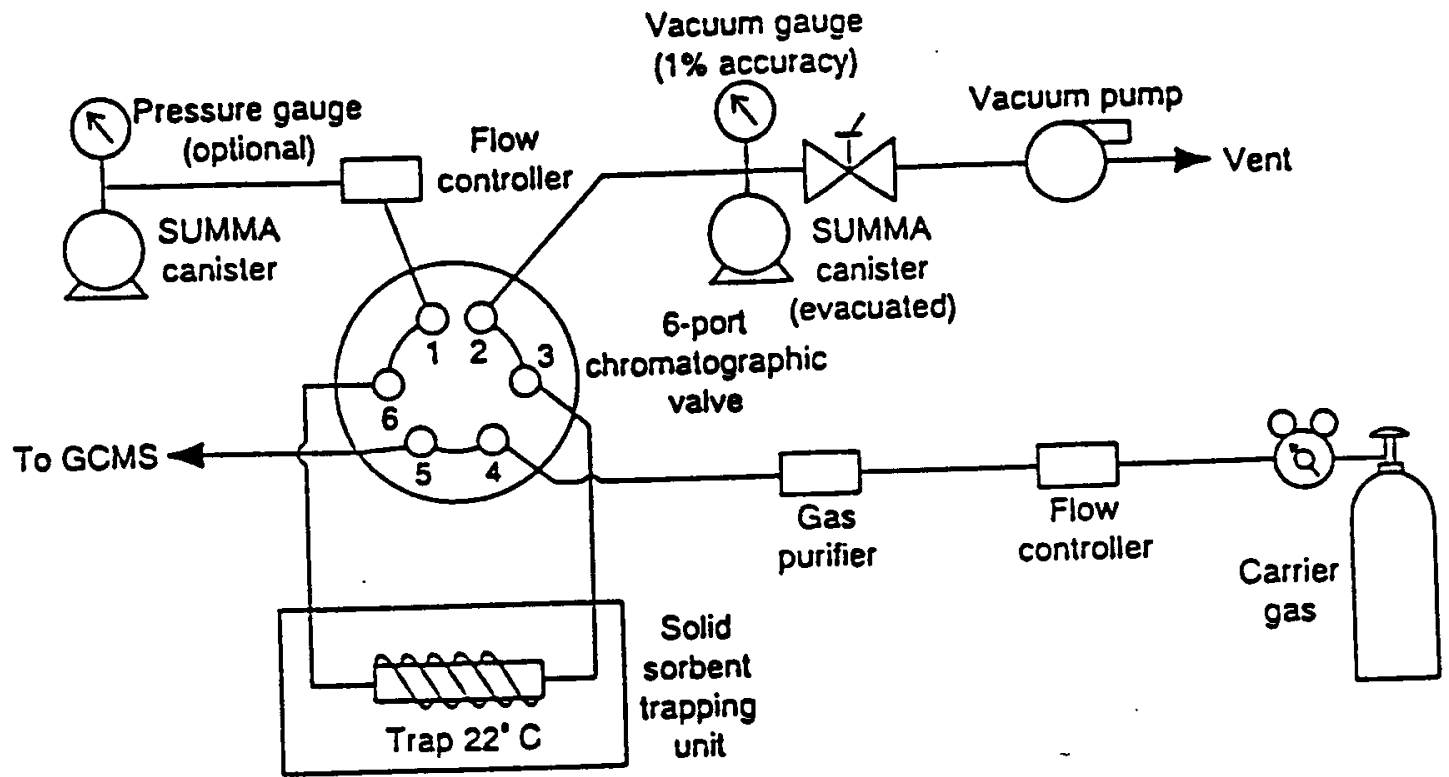

A. Sample trapping mode.

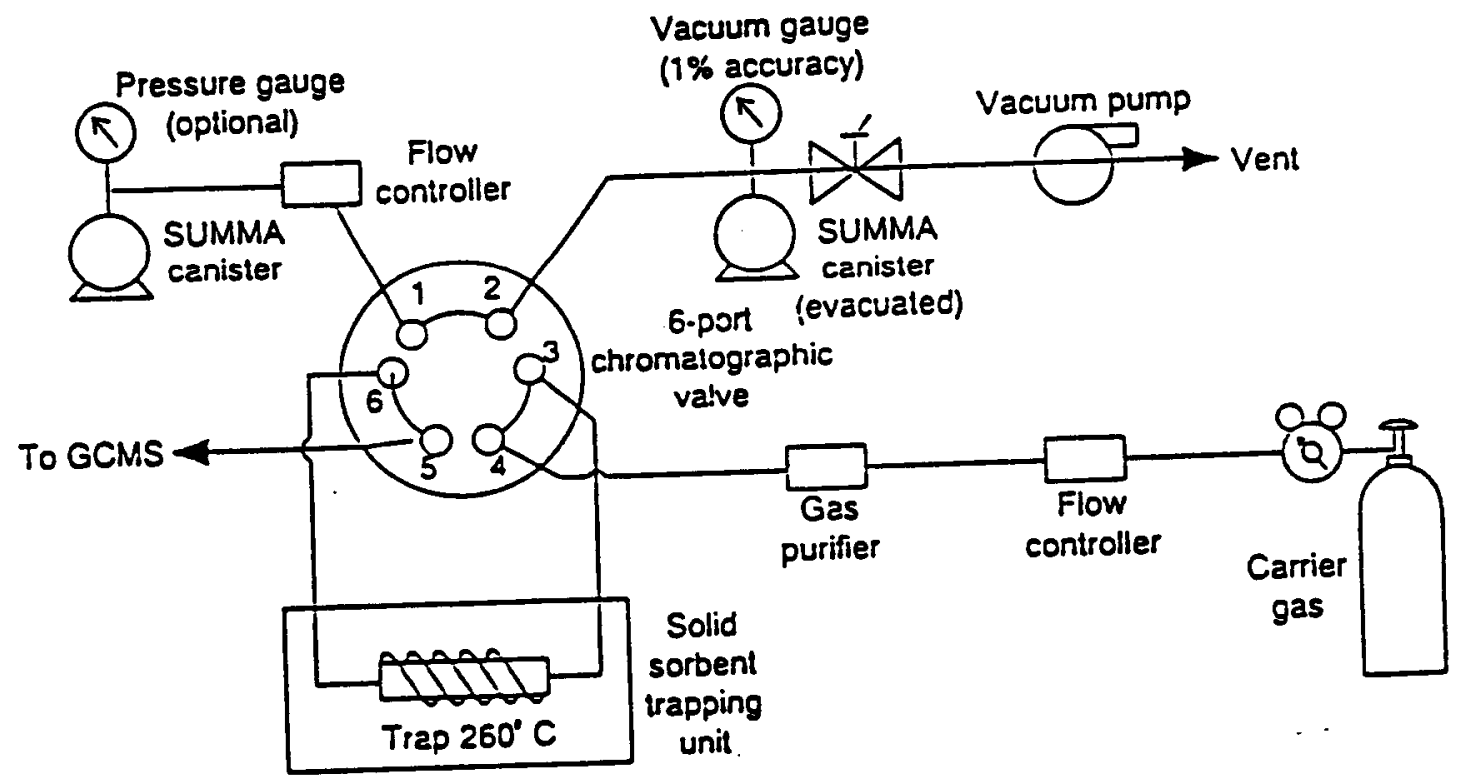

B. Sample desorption moos.

Figure 6. Solid Sorbent Trapping With Evacuated Canister 
4.1.2.5 Valve: A six-port gas chromatographic valve (Valco Instruments, Model EC6VWE or equivalent).

\subsubsection{Regulator: A stainless steel, two-stage cylinder regulator with pressure} gauges for helium GC carrier gas.

\subsection{Canister Cleaning System (Figure 7)}

4.2.1 Commercial Canister Cleanino System: A complete canister cleaning system is commercially available from Scientific Instruments Specialist, Moscow, ID. Model GS-ERS-A4.

\subsubsection{In-House Canister Cleaning System}

4.2.2.1 Vacuum pump: A vacuum pump capable of evacuating sample canister(s) to an absolute pressure of $<0.05 \mathrm{~mm} \mathrm{Hg}$.

4.2.2.2 Manifold: Manifold constructed of stainless steel tubing with connections for simultaneously cleaning several canisters.

4.2.2.3 Valves: Bellows-type shut-off valves made of stainless steel (Nupro "H" series or equivalent).

4.2.2.4 Trap: Cryogenic, open tubular U-shaped trap made of stainless steel that is cooled with liquid nitrogen to prevent contamination from back diffusion of vacuum pump oil.

4.2.2.5 Humidifier: Pressurizable water bubbler (humidifier) containing ASTM Type II water or equivalent, capable of providing moisture to the zero air or nitrogen supply.

4.2.2.6 Oven: Isothermal oven (optional) for heating canisters.

\subsection{Sample and Calibration Standard Preparation Manifold (Figure 1)}

4.3.1 Vacuum pump: A vacuum pump capable of evacuating sample canister(s) to an absolute pressure of $<0.05 \mathrm{~mm} \mathrm{Hg}$. 
Revision: 0

Date: June 14, 1991

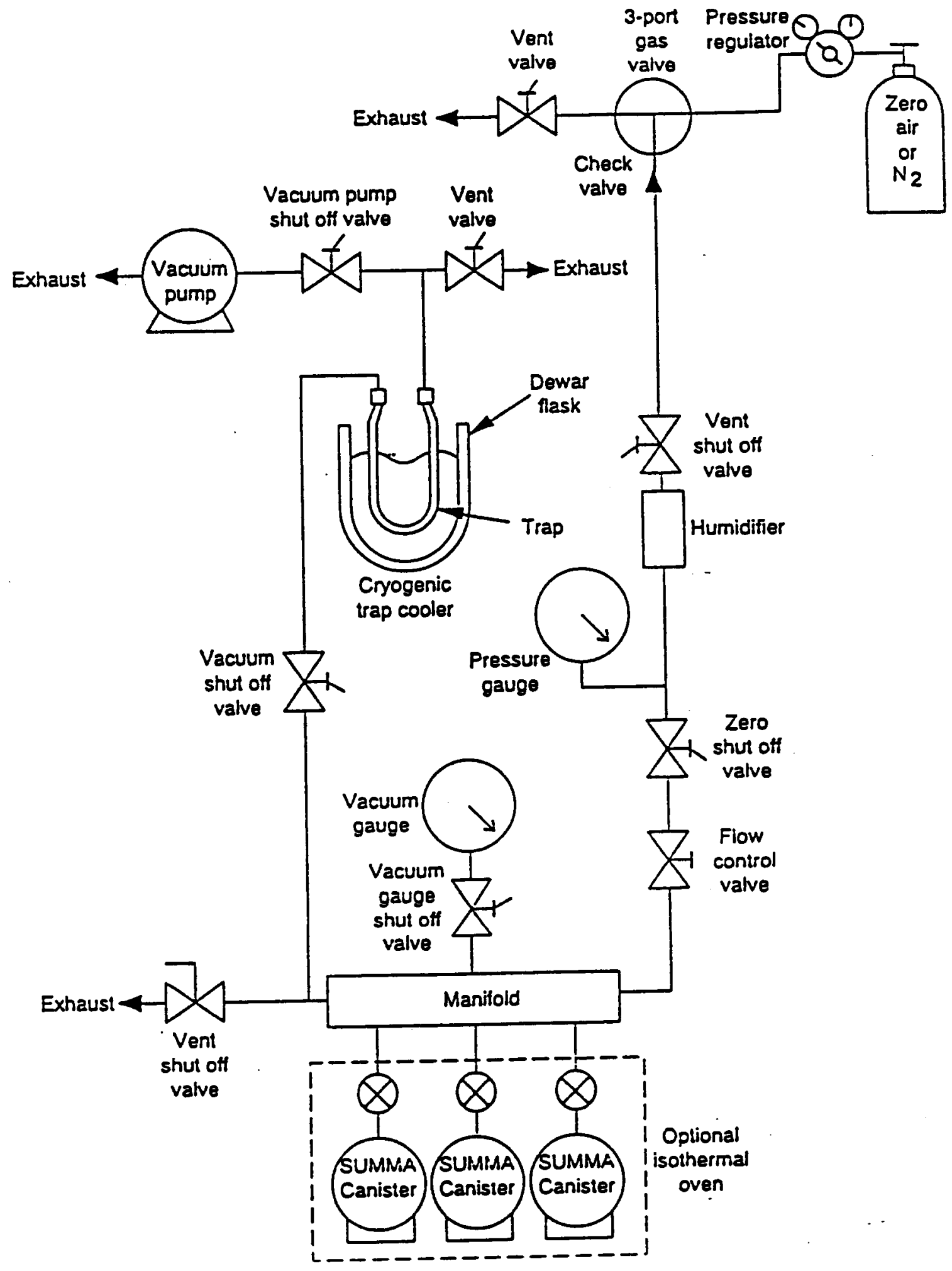

Figure 7. Canister Cleaning System 
4.3.2 Manifold: Manifold constructed of 0.25 chromatographic grade stainless steel tubing (Scott Specialty Gas Aculife treated tubing - optional) and stainless steel fittings. All tubing should be capable of being heated to $90^{\circ} \mathrm{C}$.

4.3.3 Flow controllers: One (1) 0 to $100 \mathrm{~L} / \mathrm{min}$ electronic mass flow controller and one (1) 0 to $1 \mathrm{~L} / \mathrm{min}$ electronic mass flow controller capable of indicating total flow through the controller (Matheson Model 8200-0422 with totalizer or equivalent).

4.3.4 Pressure/vacuum sensors: Two (2) pressure/vacuum sensors capable of indicating vacuum down to $0.01 \mathrm{~mm} \mathrm{Hg}$ and pressure up to $2500 \mathrm{~mm} \mathrm{Hg}$ with $1 \%$ accuracy (MKS Baratron Model or equivalent).

4.3.5 Vacuum gauge: Vacuum gauge capable of indicating vacuum down to 0.01 $\mathrm{mm} \mathrm{Hg}$.

4.3.6 Solenoid valve: Three-way magnelatch or solenoid valve (Scientific Instrument Specialists or equivalent).

4.3.7 Valves: Bellows-type shut-off valves made of stainless steel (Nupro " $H$ " series or equivalent).

4.3.8 Chronometer: Time meter capable of measuring elapsed time (Scientific Instrument Specialist Model SCT-1c or equivalent).

4.3.9 Humidifier: Pressurizable water bubbler (humidifier) containing ASTM Type Il water or equivalent capable of providing moisture to the zero air, nitrogen or calibration gases.

4.3.10 Sparge vessel: Sparge vessel capable of holding $5 \mathrm{ml}$ of ASTM Type II water, being heated to $70^{\circ} \mathrm{C}$, and being purged with zero air or nitrogen.

\subsection{REAGENTS}

5.1 Carrier and Dilution Gases: Gas cylinders of helium, nitrogen and zero air; all ultra-high purity grade. 


\subsection{Stock Standards}

5.2.1 Gases: Stock calibration gas standards of all analytes listed in Table 1 may be purchased commercially (Scott Specialty Gases or equivalent), or prepared according to U.S. EPA Compendium Method T0-2, "Determination of Volatile Organic Compounds in Ambient Air by Carbon Molecular Sieve Adsorption and Gas Chromatography/Mass Spectrometry." The standards should be traceable to a NIST Standard Reference Material (SRM) or to a NIST/EPA approved Certified Reference Material (CRM). All gas standards must be replaced after 12 months if comparison with check standards indicates a problem.

5.2.2 Liquids: Stock solutions may be prepared from pure standard materials or purchased as certified solutions. Prepare stock solutions in propanol.

5.2.2.1 Place about $9.8 \mathrm{ml}$ of propanol in a $10 \mathrm{~mL}$ tared ground glass-stoppered volumetric flask. Propanol is used only if methanol is to be determined by this method. Allow the flask to stand, unstoppered for about $10 \mathrm{~min}$ or until all alcohol-wetted surfaces have dried. Weigh the flask to the nearest $0.1 \mathrm{mg}$.

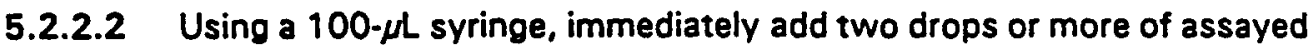
reference material to the flask and reweigh. The liquid must fall directly into the alcohol without contacting the neck of the flask. Alternatively, the density of the reference material may be used to determine the volume of material to be added to the flask.

5.2.2.3 Dilute to volume, stopper, and then mix by inverting the flask several times. Calculate the concentration in micrograms per milliliter $(\mu \mathrm{g} / \mathrm{mL})$ from the net gain in weight. When compound purity is $96 \%$ or greater, the weight may be used without correction to calculate the concentration of the stock standard. Commercially prepared stock standards may be used at any concentration if they are certified by the manufacturer or by an independent source.

5.2.2.4 Transfer the standard stock solution into a Teflon-sealed screw cap bottle. Store with minimal headspace at $-10^{\circ} \mathrm{C}$ to $20^{\circ} \mathrm{C}$ and protect from direct light.

5.2.2.5 All standards must be replaced every six months or sooner if comparison with check standards indicates a problem. 


\subsection{Secondary Dilution Standards}

\subsubsection{Calibration Standards: Using the stock calibration gas standards, prepare} secondary dilution standards containing all the analytes in Table 1 in SUMMA canisters. Alternatively, a stock solution containing all the analytes listed in Table 1 can be directly injected into an evacuated SUMMA canister followed by dilution with humid zero air or nitrogen.

Calibration standards must be prepared at a minimum of five concentration levels by dilution of stock standards. The low concentration standard must be prepared near the PROLs specified in Table 1. The four remaining standards should define the linear working range of the GCMS system. Each calibration standard should contain all the analytes listed in Table 1. All secondary dilution calibration standards must be replaced after 30 days or sooner if comparison with check standards indicates a problem.

The following is an example of how external secondary dilution calibration gas standards or internal secondary dilution calibration gas standards might be prepared using the optional calibration standard preparation manifold (Figure 1). This procedure is intended for guidance purposes only, and is based on the following assumptions:

1. The system is calibrated in nanograms instead of ppmv. This allows the determination of sample concentrations in ppmv when variable sample volumes are delivered to the GCMS.

2. The volume of a real (field) sample needed to meet PRQLs is $10 \mathrm{~mL}$.

3. The stock calibration gas standard cylinder $(\mathrm{s})$ are at $20 \mathrm{mg} / \mathrm{m}^{3}\left(\mathrm{mg} / \mathrm{m}^{3}=\mu \mathrm{g} / \mathrm{L}=\mathrm{ng} / \mathrm{mL}\right)$.

4. The volume of the secondary dilution standard to be delivered to the GCMS is $300 \mathrm{~mL}$.

5. The final secondary dilution calibration canister pressure will be three times the barometric pressure. This is equivalent to a total gas volume added to an evacuated canister of three canister volumes.

Using benzene as an example, the amount $(\mathrm{ng}$ l of benzene contained in a $10 \mathrm{~mL}$ aliquot of $1 \mathrm{ppmv}$ benzene standard can be determined as follows:

$$
p p m v=\frac{(R)(T)}{(P)(M H)} \frac{(m g)}{\left(m^{3}\right)}
$$


where

$$
\begin{aligned}
& \mathrm{R}=\text { Universal gas constant, } \mathrm{L} \text { atm mol } \\
& T=\text { Kemperature, } \mathrm{K} \\
& \mathrm{MW}=\text { Molecular weight of compound, } \mathrm{g} \mathrm{\textrm {mol } ^ { - 1 }} \\
& \mathrm{P} \quad=\text { Pressure, atm } \\
& \text { At } 25^{\circ} \mathrm{C} \text { and } 1 \mathrm{~atm}, \mathrm{RT} / \mathrm{P}=24.5 \mathrm{~L} \mathrm{~mol}^{-1}
\end{aligned}
$$

Therefore, $1 \mathrm{ppmv}$ of benzene (MW $=78.1 \mathrm{~g} \mathrm{~mol}^{-1}$ ) contains approximately $0.0032 \mathrm{~g}$ benzene $/ \mathrm{m}^{3}$ or $3.2 \mathrm{ng}$ benzene $/ \mathrm{mL}$, and a $10 \mathrm{~mL}$ aliquot would deliver about $32 \mathrm{ng}$ of benzene.

This shows that the amount of benzene needed to be delivered to the GCMS for the low concentration standards is about $32 \mathrm{ng}$. The amount of volume used to deliver the $32 \mathrm{ng}$ is not important, what is important is that sufficient gas volume be used in order to minimize analytical error.

Assumptions (4) and (5) can be coupled with the $32 \mathrm{ng}$ of benzene to determine how many total nanograms of benzene need to be transferred from the stock calibration gas cylinder(s) to the evacuated $2.8 \mathrm{~L}$ canister. The $2.8 \mathrm{~L}$ canister will contain a total of $8.4 \mathrm{~L}$ of gas. The amount of benzene needed for calibration is $32 \mathrm{ng}$ in $300 \mathrm{~mL}$. Therefore, the total amount of benzene in the canister is $(32 \mathrm{ng} / 0.30 \mathrm{~L}) \times(8.4 \mathrm{~L})=896 \mathrm{ng}$. It has been assumed that the stock calibration gas standards are $20 \mathrm{mg} / \mathrm{m}^{3}$ or $20 \mathrm{ng} / \mathrm{mL}$. Transferring $44.8 \mathrm{~mL}$ from the stock gas standard to the evacuated $2.8 \mathrm{~L}$ canister followed by dilution with $8.355 \mathrm{~L}$ of humid zero air or nitrogen will produce a secondary dilution standard that has $32 \mathrm{ng} / 300 \mathrm{~mL}$ of benzene. Delivery of $300 \mathrm{~mL}$ of this secondary dilution standard will yield a calibration point that is equivalent to $10 \mathrm{~mL}$ of $1 \mathrm{ppmv}$ benzene.

\subsubsection{External secondary dilution calibration standard: To prepare a $1 \mathrm{ppmv}$} benzene external calibration standard using stock calibration gas standards, refer to Figure 1.

1. Install evacuated $2.8 \mathrm{~L}$ canister in position (1).

2. Install stock gas cylinders as shown.

3. Evacuate all manifold lines up to valves (2) and (25) to $<0.05 \mathrm{~mm} \mathrm{Hg}$. Keep valves (3), (19)-(22) and (24) closed.

4. Close valves (7), (4) and (23); open valve (2), and record the initial canister vacuum. Close valves (2), (5) and (7).

5. Set calibration cylinder (34) to deliver gas at $20 \mathrm{psig.}$

6. Close valve (29) and set three-way valve (8) to vent position. 
7. Open valve (19) and set mass flow controller $(10)$ to $10 \mathrm{~mL} / \mathrm{min}$. Condition manifold tubes and stabilize mass flow controller by letting gas flow to vent for 5 minutes.

8. Set timer (9) to deliver the volume of gas needed. For a $1 \mathrm{ppmv}$ benzene standard from a $20 \mathrm{ng} / \mathrm{mL}$ stock calibration standard, this is $44.8 \mathrm{~mL}$ at $10 \mathrm{~mL} / \mathrm{min}$ which gives 4.48 minutes (269 seconds).

9. After 5 minutes of flowing to vent, switch three-way valve (8), initiate timer (9), and open valves (2) and (7). These steps should be done as quickly as possible.

10. When the timer (9) elapses, immediately close valve (2).

11. Close valve (19), open valve (29) and evacuate the manifold until the vacuum gauge (32) reads less than $0.05 \mathrm{~mm} \mathrm{Hg}$.

12. Repeat steps (4) to (11) until aliquots from all stock gas cylinders have been transferred to the $2.8 \mathrm{~L}$ SUMMA canister.

13. Set either the zero air or nitrogen cylinder to deliver gas at $60 \mathrm{psig}$. Set three-way valve (40) to vent position and set mass flow controller to $750 \mathrm{ml} / \mathrm{min}$ and stabilize flow controller for 5 minutes.

14. Close valves (18) and (7). Switch three-way valve (40), open valves (25), (23), (4) and (2).

15. Transfer humid nitrogen or zero air until the $2.8 \mathrm{~L}$ canister has the equivalent of 3 canister volumes of gas $(8.4 \mathrm{~L}$ ). The volume of dilution gas (nitrogen/zero air) is determined by 8.4 $L$ minus the amount of stock gas calibration standard added. The time required for this volume to be transferred is the volume needed divided by the flow rate. For the $1 \mathrm{ppmv}$ benzene standard, this would be $8355 \mathrm{~mL} / 750 \mathrm{~mL} / \mathrm{min}=11.14 \mathrm{~min}=668$ seconds.

16. After the required time has elapsed, immediately close valve (2). Then close valves (4), (23), and (25), and switch three-way valve (40) to vent. Close nitrogen/zero air tank valve.

17. Open valves (29), (7), and (5), and evacuate to $<0.05 \mathrm{~mm} \mathrm{Ho}$.

18. Close valve (7), open valve (2), and record final canister pressure.

5.3.1.1.1 As an alternative to using accurate time and mass flow to transfer stock gas standards, continuous pressure/vacuum monitoring can be used. This can be accomplished by modifying steps (4) to (12) as follows: 
4. Close valves (4), (7) and (23); open valve (2), and record the canister vacuum. Close valves (2) and (7).

5. Set calibration cylinder (34) to deliver gas at 20 psig.

6. Close valve (29) and set three-way valve (8) to vent position.

7. Open valve $(19)$ and set mass flow controller $(10)$ to $10 \mathrm{~mL} / \mathrm{min}$. Condition manifold tubes and stabilize mass flow controller by allowing gas flow to vent for 5 minutes.

8. Set timer (9) to deliver $25 \%$ more volume of gas then what is needed to transfer. For a 1 ppmv benzene standard, $44.8 \mathrm{~mL}$ are required; $25 \%$ above the requirement would be $56.0 \mathrm{~mL}$. At $10 \mathrm{~mL} / \mathrm{min}$, the timer would be set to 5.6 minutes (336 seconds).

9. After 5 minutes of flowing to vent, switch three-way valve (8), initiate timer (9), and open valves (7) and (2). These steps should be done as quickly as possible.

10. To determine when to close valve (7) and stop the transfer of the stock calibration gas, the desired final pressure/vacuum needs to be calculated. The pressure/vacuum at the end of transfer is calculated as follows:

$$
\frac{P_{f}-P_{1}}{P_{e}} V_{t}=V_{d}
$$

where

$$
\begin{aligned}
& P_{a}=\text { Atmospheric pressure, } \mathrm{mm} \mathrm{Hg} \\
& P_{f}=\text { Final pressure, } \mathrm{mm} \mathrm{Hg} \\
& P_{1}=\text { Initial pressure, } \mathrm{mm} \mathrm{Hg} \\
& V_{d}=\text { Volume of gas to be delivered, } \mathrm{mL} \\
& V_{t}=\text { SUMMA canister volume, } \mathrm{mL} .
\end{aligned}
$$

If $44.8 \mathrm{~mL}$ are to be delivered to a $2.8 \mathrm{~L}$ canister with an initial pressure of $0.05 \mathrm{~mm} \mathrm{Hg}$, the final pressure will be $12.2 \mathrm{~mm} \mathrm{Hg}$. Therefore, when the pressure/vacuum sensor (6) approaches 12.2 $\mathrm{mm} \mathrm{Hg}$, reduce the flow with valve (7). When the desired pressure is reached, completely close valve (7) and document the final pressure. Close valve (2) and use the above equation to determine the volume of stock calibration gas standard transferred. After closing valve (2), reset the timer and switch three-way valve (8) to the vent position.

11. Close valve (19), open valves (7) and (29), and evacuate the manifold until vacuum gauge (5) reads less than $0.05 \mathrm{~mm} \mathrm{Ho}$. 
12. Repeat steps (4) to (11) until aliquots from all stock gas cylinders have been transferred to the $2.8 \mathrm{~L}_{\text {SUMMA }}$ canister. When repeating step $(4)$ it is not necessary to record the initial pressure because this is the final pressure of the previous stock calibration gas standard transfer. When repeating step (9), it is important that the pressure read at sensor $(6)$ be greater than the final pressure of the previous transfer before opening valve (2).

5.3.1.2 Internal standard secondary dilution calibration standard: The preparation of a 1 ppmv internal standard is identical to the preparation of an external standard except for the addition of internal standards. In this procedure, internal standards are added to the $2.8 \mathrm{~L}$ SUMMA canister after transferring all stock calibration gas standards and prior to the final dilution with humid zero air or nitrogen. Several procedures may be used to introduce internal standards into calibration standard and/or sample canisters:

1. Direct injection of a stock solution of internal standards into the canister

2. Direct gas transfer from a stock gas standard into the canister

3. Injecting the internal standards into ASTM Type II water followed by purging them into the canister with nitrogen or ultra air at $100 \mathrm{~mL} / \mathrm{min}$ for 10 minutes while maintaining the sparge vessel at $70^{\circ} \mathrm{C}$.

The amount (ng) of each internal standard added to the $2.8 \mathrm{~L}$ canister must give $250 \mathrm{ng}$ per secondary dilution gas standard aliquot $(300 \mathrm{~mL})$ delivered to the GCMS. For a $2.8 \mathrm{~L}$ canister with a total gas volume of $8.4 \mathrm{~L}$ and using a $300 \mathrm{~mL}$ aliquot, a total of $7000 \mathrm{ng}$ of each internal standard must be added to each calibration standard canister. Alternatively, internal standards may be added directly to the trap prior to calibration standard or sample loading. If this procedure is employed, $250 \mathrm{~mL}$ of a gas standards containing the internal standard should be used to deliver the $250 \mathrm{ng}$ of each internal standard.

5.3.2 Laboratory Control Samples: Laboratory control samples (LCSs) are described in Section 8.4. The preparation of LCSs is identical to the preparation of calibration standards.

5.4 Internal Standards: The recommended internal standards are fluorobenzene and chlorobenzene- $d_{5}$. Prepare internal standard stock and secondary dilution standards in propanol if methanol is to be analyzed by this method. Each calibration standard or sample aliquot delivered to the GCMS must have $250 \mathrm{ng}$ of each internal standard. 
5.5 4-Bromofluorobenzene (BFB) Standard: A standard solution containing $25 \mathrm{ng} / \mathrm{mL}$ of BFB in propanol (if methanol is to be analyzed by this method) should be prepared if direct injection is used. A gas standard may be purchased commercially (Scott specialty Gases or equivalent) if gas introduction is used. Alternatively, a gas standard may be prepared according to Section 5.3.1.2. The concentrations of gas standards must be such that a total of $50 \mathrm{ng}$ is delivered to the GCMS.

5.6 Reagent Water: Reagent water is defined as water in which an interferant is not observed at the method detection limit (MDL) of the parameters of interest. Reagent water which is generated must meet the performance specifications for ASTM Type II water. To generate reagent water, either:

1. Pass tap water through a carbon filter bed containing $500 \mathrm{~g}$ of activated carbon (Calgon Corp., Filtrasorb-300 or equivalent); or,

2. Pass the water through a purification system (Millipore Super-Q or equivalent); or,

3. Boil tap water for 15 minutes and then, while maintaining the water temperature at $85^{\circ} \mathrm{C}$ to $95^{\circ} \mathrm{C}$, bubble a contaminant-free inert gas through the water for one hour. While still hot, transfer the water to a narrow mouth screw-top bottle seal with a Teflon-lined septum and cap.

\subsection{SAMPLE COLLECTION, PRESERVATION AND HANDLING}

6.1 Samples will be collected using the sampling procedures described in the WIPP Experimental-Waste Characterization Program QAPP.

6.2 Sample canisters must be stored at room temperature. Sample holding times cannot exceed 28 days.

6.3 All sample handling and chain-of-custody procedures described in the QAPP for the WIPP Experimental-Waste Characterization Program must be followed.

\subsection{PROCEDURE}

\subsection{Recommended GCMS Operating Conditions}

Electron energy:

Mass range:

Scan time:

Column type:
70 volts (nominal)

29-260 amu

One second/scan or enough to give 5 scans/peak

DB-624, $30 \mathrm{~m} \times 0.53 \mathrm{~mm}$ l.D. 
Initial column temperature: $\quad 10^{\circ} \mathrm{C}$ Initial column holding time: 5 minutes

Column temperature program: Final column temperature: $6^{\circ} \mathrm{C}$ per minute $160^{\circ} \mathrm{C}$

Final column holding time: Until all compounds have eluted Injector temperature:

Source temperature:

Transfer line temperatures:

Carrier gas: $200 \cdot 225^{\circ} \mathrm{C}$

According to manufacturer specifications $250-300^{\circ} \mathrm{C}$ Helium at $15 \mathrm{~mL} / \mathrm{min}$

7.2 Analytical System Options: Four possible inlet/trapping GCMS systems are shown in Figures 4 to 7. The hardware requirements for these systems have been given in Section 4.1.

7.2.1 Cryogenic Trapping: Mass flow controller and evacuated canister systems for delivery and measurement of sample volumes are shown in Figures 4 and 5.

7.2.1.1 Mass flow controller system: The analytical system should be properly assembled using Figure 4 for guidance and certified using humid air or nitrogen (Section 8.8).

7.2.1.1.1 Mass flow controllers should be checked and adjusted to provide correct flow rates for the system. The small diaphragm pump is used to maintain a constant pressure differential across the mass flow controller.

7.2.1.1.2 The sample canister is connected to the system as shown in Figure 4. Calibration standards and samples that have been diluted by a factor of 30 or more are delivered to the cryogenic trap at $30 \mathrm{~mL} / \mathrm{min}$. Samples that have not been diluted to this extent can still be analyzed with this system but the mass flow controller needs to be set to deliver the sample over 10 minutes. Sub-ambient pressure samples can also be analyzed by this procedure.

7.2.1.1.3 With the system in the desorb/precondition mode (Figure 4b), the GC oven and cryogenic trap are set to their initial set points of $10^{\circ} \mathrm{C}$ and $-160^{\circ} \mathrm{C}$, respectively.

7.2.1.1.4 When the cryogenic trap reaches its set point, the canister valve is opened and the transfer lines and mass flow controller are conditioned and stabilized.

7.2.1.1.5 After one to two minutes, the six-way chromatographic valve is turned to its fill (trapping) position (Figure 4a). 
7.2.1.1.6 A ten-minute collection period of canister sample is utilized. [Note: $30 \mathrm{~mL} / \mathrm{min} \times 10 \mathrm{~min}=300 \mathrm{~mL}$ sampled]. After 10 minutes, the sample canister valve is closed.

7.2.1.1.7 After the sample is preconcentrated in the cryogenic trap, the six-way chromatographic valve is cycled to the inject position and the cryogenic trap is heated. The trapped analytes are thermally desorbed onto the head of the DB-624 capillary column.

7.2.1.1.8 Upon sample desorption, the GCMS data system is signaled to begin mass spectrometric data acquisition and to begin the chromatographic temperature program. Qualitative and quantitative identification procedures will be discussed under Data Interpretation (Section 7.8).

\subsubsection{Evacuated canister system: The analytical system should be properly} assembled using Figure 5 for guidance and certified using humid zero air or nitrogen (Section 8.8). This system should be employed when the available volume of sample is limited and it is not possible to deliver $300 \mathrm{~mL}$.

7.2.1.2.1 The volume of the evacuated canister used to collect the trap effluent should be known to within $2 \%$ of the actual volume.

7.2.1.2.2 The change in pressure of the evacuated canister as trap effluent is collected is used to determine how much sample has been delivered to the trap. The volume of gas delivered can be calculated using equation (1) from Section 5.3.1.2.

7.2.1.2.3 The sample canister and evacuated canister are connected as shown in Figure 5. The flow controller is used to regulate sample flow so that the desired final pressure is achieved.

7.2.1.2.4 With the system in the sample trapping mode (Figure 5a), the GC oven and cryogenic trap are set to their initial set points of $10^{\circ} \mathrm{C}$ and $-160^{\circ} \mathrm{C}$, respectively.

7.2.1.2.5 When the cryogenic trap reaches its set point, the trap vent valve is closed isolating the vacuum pump. 
7.2.1.2.6 The pressure in the evacuated canister is measured and recorded.

7.2.1.2.7 The isolation valves on both canisters are opened allowing sample to flow through the trap and into the evacuated canister.

7.2.1.2.8 The sample canister valve is closed when the evacuated canister pressure indicates the desired volume of sample has been delivered.

7.2.1.2.9 After the sample is preconcentrated in the cryogenic trap, the six-way chromatographic valve is cycled to the inject position and the cryogenic trap is heated. The trapped analytes are thermally desorbed onto the head of the DB-624 capillary column.

\subsection{Same as Section 7.2.1.1.8.}

7.2.2 Solid Sorbent Trapping: Mass flow controller and evacuated canister systems for delivery and measurement of sample volumes are shown in Figures 6 and 7. If a commercial purgeand-trap system or equivalent is used (Tekmar LSC-2/ALS), then the vacuum pump and mass flow controller are connected to the trap vent bulk head fitting and the sample canister is connected to the purge flow inlet on the LSC-2 or the ALS. If connected to the ALS with $1 / 16$ inch stainless steel heated transfer line, the line is connected to the purge flow outlet.

7.2.2.1 Mass flow controller system: The analytical system should be properly assembled using Figure 6 for guidance and certified using humid zero air or nitrogen (Section 8.8).

\subsection{Same as Section 7.2.1.1.1.}

\subsection{Same as Section 7.2.1.1.2, except reference Figure 6.}

7.2.2.1.3 With the system in the desorb/precondition mode (Figure 6b), the GC oven and solid sorbent trap are set to their initial set points of $10^{\circ} \mathrm{C}$ and $30^{\circ} \mathrm{C}$, respectively.

7.2.2.1.4 When the solid sorbent trap reaches its set point, the canister valve is opened and the transfer line and mass flow controller are conditioned and stabilized. This step is omitted when a commercial purge-and-trap system is used. 
7.2.2.1.5 After one to two minutes, the six-way chromatographic valve is turned to its fill (trapping) mode (Figure 5a). With a commercial purge-and-trap, the system would be set to the purge mode and the sample canister would be opened.

\subsection{Same as Section 7.2.1.1.6.}

7.2.2.1.7 Same as Section 7.2.1.1.7, except a solid sorbent trap is used. Using commercial purge-and-trap hardware, the system would advance to the desorb mode after the time set for purging had elapsed.

\subsection{Same as Section 7.2.1.1.8.}

\subsubsection{Evacuated canister system: Same as Section 7.2.1.2, except reference}

Figure 7. If a commercial purge-and-trap system is used, the vacuum pump and evacuated canister are connected to the trap vent bulkhead fitting and the sample canister is connected to the purge flow inlet on the LSC-2 or the ALS.

\subsection{Same as Section 7.2.1.2.1.}

\subsection{Same as Section 7.2.1.2.2}

7.2.2.2.3 Same as Section 7.2.1.2.3, except reference Figure 7.

7.2.2.2.4 With the system in the sample trapping mode (Figure 7a), the GC oven and solid sorbent trap are set to their initial temperature set points of $10^{\circ} \mathrm{C}$ and $30^{\circ} \mathrm{C}$, respectively. A commercial purge-and-trap system would be in the purge mode.

7.2.2.2.5 When the solid sorbent trap reaches its set point, the trap vent valve is closed isolating the vacuum pump.

7.2.2.2.6 The pressure in the evacuated canister is measured and recorded.

7.2.2.2.7 Same as Section 7.2.1.2.7.

7.2.2.2.8 Same as Section 7.2.1.2.8. 
7.2.2.2.9 Same as Section 7.2.2.1.9, except that the solid sorbent trap is heated to $220^{\circ} \mathrm{C}$. A commercial purge-and-trap system would be advanced to the desorb mode.

\subsection{Same as Section 7.2.1.1.8.}

7.3 GCMS Instrument Performance Testing: Every 12 hours or prior to a calibration, it must be demonstrated that the GCMS system meets the BFB criteria specified in Table 2. Instrument hardware tuning may be performed with perfluorotributylamine (PFTBA). Instrument performance criteria must be met by putting $50 \mathrm{ng}$ of BFB on-column. The BFB may be introduced to the GCMS system via a gas standard or a liquid standard.

7.4 Initial Calibration Procedures: Initially, a five-point calibration is performed on the GCMS system. Section 5.0 provides guidance on how to prepare both internal and external standard calibration mixtures at the required concentration (ng) levels. Sections 7.1 and 7.2 provide suggested GCMS operating parameters and GCMS analytical system options. After the secondary dilution gas standards have been prepared and the GCMS analytical system has been configured, initial calibration can begin. Prior to initial calibration, the system must be certified as clean by passing the humidified air or nitrogen test (Section 8.8).

\subsubsection{Internal Standard Calibration}

7.4.1.1 Tabulate the area response of the characteristic ions (Table 3) against the amount (ng) for each compound and for each internal standard for the five calibration standards. Calculate the relative response factor (RRF) for each compound using the assignments made in Table 4. When the analytical system is calibrated in nanograms, the RRF is calculated as follows:

$$
R R F=\frac{\left(A_{x} C_{L}\right)}{\left(A_{\Delta} C_{\nu}\right)}
$$

where

$A_{x}=$ area of the characteristic ion for the compound being measured

$A_{1 S}=$ area of the characteristic ion for the specific internal standard

$C_{1 S}=$ amount of the specific internal standard, $n g$

$C_{x}=$ amount of the compound being measured, $n g$. 
Table 3. Characteristic lons for Volatile Organic Compounds

\begin{tabular}{|c|c|c|}
\hline Volatiles & Primary lon & Secondary Ion \\
\hline $\begin{array}{l}\text { 1. Acetone } \\
\text { 2. Benzene } \\
\text { 3. Bromoform } \\
\text { 4. 1-Butanol } \\
\text { 5. 2-Butanone }\end{array}$ & $\begin{array}{r}43 \\
78 \\
173 \\
56 \\
72\end{array}$ & $\begin{array}{c}58 \\
52,77 \\
171,175,252 \\
31 \\
57,43\end{array}$ \\
\hline $\begin{array}{l}\text { 6. Carbon Tetrachloride } \\
\text { 7. Chlorobenzene } \\
\text { 8. Chloroform } \\
\text { 9. Cyclohexane } \\
\text { 10. 1,1-Dichloroethane }\end{array}$ & $\begin{array}{r}117 \\
112 \\
83 \\
56 \\
63\end{array}$ & $\begin{array}{c}119,121 \\
114,77 \\
85,47 \\
65,83\end{array}$ \\
\hline $\begin{array}{l}\text { 11. 1,2-Dichloroethane } \\
\text { 12. 1,1-Dichloroethene } \\
\text { 13. cis-1,2-Dichloroethene } \\
\text { 14. Ethyl Benzene } \\
\text { 15. Ethyl Ether }\end{array}$ & $\begin{array}{r}63 \\
96 \\
96 \\
106 \\
31\end{array}$ & $\begin{array}{c}65,83 \\
61,98 \\
61,98 \\
91 \\
59\end{array}$ \\
\hline $\begin{array}{l}\text { 16. Methanol } \\
\text { 17. Methylene Chloride } \\
\text { 18. 4-Methyl-2-pentanone } \\
\text { 19. 1,1,2,2-Tetrachloroethane } \\
\text { 20. Tetrachloroethene }\end{array}$ & $\begin{array}{r}31 \\
84 \\
43 \\
83 \\
164\end{array}$ & $\begin{array}{c}49,51,86 \\
58,100 \\
85,131,166 \\
129,131,166\end{array}$ \\
\hline $\begin{array}{l}\text { 21. Toluene } \\
\text { 22. 1,1,1-Trichloroethane } \\
\text { 23. Trichloroethene } \\
\text { 24. 1,1,2-Trichloro-1,2,2- } \\
\text { trifluoroethane } \\
\text { 25. 1,3,5-Trimethylbenzene }\end{array}$ & $\begin{array}{r}92 \\
97 \\
130 \\
101 \\
105\end{array}$ & $\begin{array}{c}91,65 \\
99,117 \\
95,97,132 \\
155,85 \\
120\end{array}$ \\
\hline $\begin{array}{l}\text { 26. 1,2,4-Trimethylbenzene } \\
\text { 27. m-Xylene } \\
\text { 28. o-Xylene } \\
\text { 29. p-Xylene } \\
\text { 30. Chlorobenzene-d } d_{5} \text { (IS) } \\
\text { 31. Fluorobenzene (IS) }\end{array}$ & $\begin{array}{r}105 \\
106 \\
106 \\
106 \\
117 \\
96\end{array}$ & $\begin{array}{c}120 \\
91 \\
91 \\
91 \\
82,119 \\
77\end{array}$ \\
\hline
\end{tabular}


Table 4. Volatile Organic Internal Standards

With Corresponding Analytes

Assigned for Quantitation

\begin{tabular}{ll}
\hline & \\
\hline Fluorobenzene & Chlorobenzene-d 5 \\
\hline Acetone & Bromoform \\
Benzene & Chlorobenzene \\
1 -Butanol & Ethylbenzene \\
2-Butanone & 4-Methyl-2-pentanone \\
Carbon Tetrachloride & $1,1,2,2-$ Tetrachloroethane \\
Chloroform & Tetrachloroethene \\
Cyclohexane & Toluene \\
$1,1-$ Dichloroethane & $1,3,5-$ Trimethylbenzene \\
$1,2-$ Dichloroethane & $1,2,4-$ Trimethylbenzene \\
$1,1-$ Dichloroethene & m-Xylene \\
cis-1,2-Dichloroethene & $0-$ Xylene \\
Ethyl Ether & p-Xylene \\
Methanol & \\
Methylene Chloride & \\
$1,1,1-$ Trichloroethane & \\
Trichloroethene & \\
$1,1,2-$ Trichloro-1,2,2-trifluoroethane & \\
\hline
\end{tabular}


7.4.1.2 The average RRF must also be calculated for each compound. Using the RRFs, calculate the percent relative standard deviation (\% RSD) for all compounds listed in Table 1. The \%RSD is calculated as follows:

$$
\% R S D=\frac{S}{\bar{X}} \times 100
$$

where

$$
\begin{aligned}
& \bar{X}=\text { mean of } 5 \text { initial RRFs for a compound = average RRF } \\
& S=\text { standard deviation of RRFs for a compound. }
\end{aligned}
$$

The \%RSD for each individual compound must be less than $35 \%$. For those compounds having a \%RSD greater than $35 \%$, the GCMS data system may be used to generate a second or third order regression calibration curve. The initial calibration reporting form (WIPP Experimental-Waste Characterization Program QAPP) must indicate when higher order calibration curves are utilized. Sample analysis cannot proceed until the \%RSD requirement for each compound is met.

\subsubsection{External Standard Calibration}

7.4.2.1 Tabulate the area response of the characteristic ions (Table 3) against amount (ng) for each compound from the five calibration standards. Calculate the response factor (RF) for each compound. When the analytical system is calibrated in nanograms, the RF is calculated as follows:

$$
R F=\frac{A_{x}}{C_{x}}
$$

where

$$
\begin{aligned}
& A_{x}=\text { area of the characteristic ion for the compound being measured } \\
& C_{x}=\text { amount of the compound being measured, } n g .
\end{aligned}
$$

7.4.2.2 The average RF and the \%RSD must be calculated for all compounds listed in Table 1. The \%RSD is calculated as in Section 7.4.1.2. The same acceptance criteria apply to RFs as to RRFs (Section 7.4.1.2). 


\subsection{Daily GCMS Calibration}

7.5.1 Prior to the analysis of any samples (including standards), instrument performance criteria must be satisfied (Section 7.3).

7.5.2 The initial calibration curve (Section 7.4) for each compound listed in Table 1 must be checked and verified once during every 12-hour shift and prior to the analysis of any samples. This is accomplished by analyzing a calibration standard that contains amounts (ng) of compounds near the midpoint amounts of the initial calibration.

7.5.3 The verification procedure for internal and external calibration standards is identical. The validity of the initial calibration curve is checked by calculating the percent difference for each compound as follows:

$$
\% D=\frac{\left(R F_{l}\right)-\left(R F_{d}\right)}{\left(R F_{j}\right)} \times 100
$$

where

$$
\begin{aligned}
& \% D=\text { percent difference } \\
& R F_{1}=\text { average response factor or average relative response factor from initial calibration } \\
& R F_{c}=\text { response factor or relative response factor from current midpoint standard. }
\end{aligned}
$$

The percent difference for all compounds must be less than $30 \%$. For those compounds where a higher order regression curve was used, the point from the continuing calibration standard for the compound must fall within $30 \%$ of the curve value from the initial calibration. If the $\% \mathrm{D}$ criterion is not met, corrective action must be taken. If no source of the problem can be determined after corrective action has been taken, a new five-point calibration must be generated. This criterion must be met before quantitative sample analysis begins.

7.5.4 The internal standard area responses in the continuing calibration standard must be evaluated immediately after or during sample analysis. If the retention time of any internal standard changes by more than 30 seconds from the last continuing calibration, the chromatographic system must be checked for malfunctions and corrections must be made. If the EICP area for any internal standard changes by a factor of two from the last continuing calibration standard, the GCMS system must be checked for malfunctions and corrections must be made. Samples analyzed while the system was malfunctioning must be reanalyzed. 


\subsection{Sample Analysis}

7.6.1 Figure 8 illustrates the sequence of events that should occur at the laboratory upon receipt of a sample canister. Details of chain-of-custody requirements and specific forms are given in the WIPP Experimental-Waste Characterization Program QAPP. The $250 \mathrm{~mL}$ SUMMA* canisters used to collect the headspace samples have pressure/vacuum gauges attached. The canister pressure as received from the field must be documented.

7.6.2 As discussed in Section 5.3, the minimum volume of sample required to meet the PROLs specified in Table 1 is $10 \mathrm{~mL}$. The analytical systems described in Section 7.2 are for sample volumes greater than $20 \mathrm{~mL}$. Sections 7.2.1.1 and 7.2.2.1 describe analytical systems that can be used when the volume of gas delivered is $>100 \mathrm{~mL}$. Sections 7.2 .1 .2 and 7.2.2.2 describe analytical systems that can be used when the volume of gas delivered is in the range of $20-80 \mathrm{~mL}$. Given that a $10 \mathrm{~mL}$ sample aliquot is sufficient to meet the PROLs but not sufficient for the analytical systems described, it will be necessary to dilute the sample prior to analysis. Section 5.3 presents procedures for transferring and diluting gas samples.

7.6.3 Figure 2 illustrates how a sample can be diluted by a factor of 30 or more. Dilution of a $10 \mathrm{~mL}$ sample by a factor of 30 would allow delivery of $300 \mathrm{~mL}$ to the analytical system and would be the same as described for calibration standards (Section 5.3). Transferring a $50 \mathrm{~mL}$ sample aliquot to a $500 \mathrm{~mL}$ evacuated canister followed by the addition of $1.45 \mathrm{~L}$ of zero air will result in a thirty-fold dilution of the sample. If the field canister is pressurized before transferring the sample aliquot, the resultant dilution must be taken into account before transferring to the $500 \mathrm{~mL}$ canister, i.e., the aliquot volume would increase. The analytical systems described in Sections 7.2.1.1 and 7.2.2.1 should be used for dilution factors of ten or greater. The analytical systems described in Sections 7.2.1.2 and 7.2.2.2 should be used for dilution factors of ten or less.

\subsubsection{Guidance for the Preparation and Analysis of a Diluted (30x) Sample}

7.6.4.1 Attach a $500 \mathrm{~mL}$ evacuated laboratory canister as shown in Figure 1.

7.6.4.2 Attach a $250 \mathrm{~mL}$ field canister as shown in Figure 1.

7.6.4.3 Evacuate the manifold to $<0.05 \mathrm{~mm} \mathrm{Hg}$ with valves (5), (7), (12), (13), (28), (18) and (29) open. 


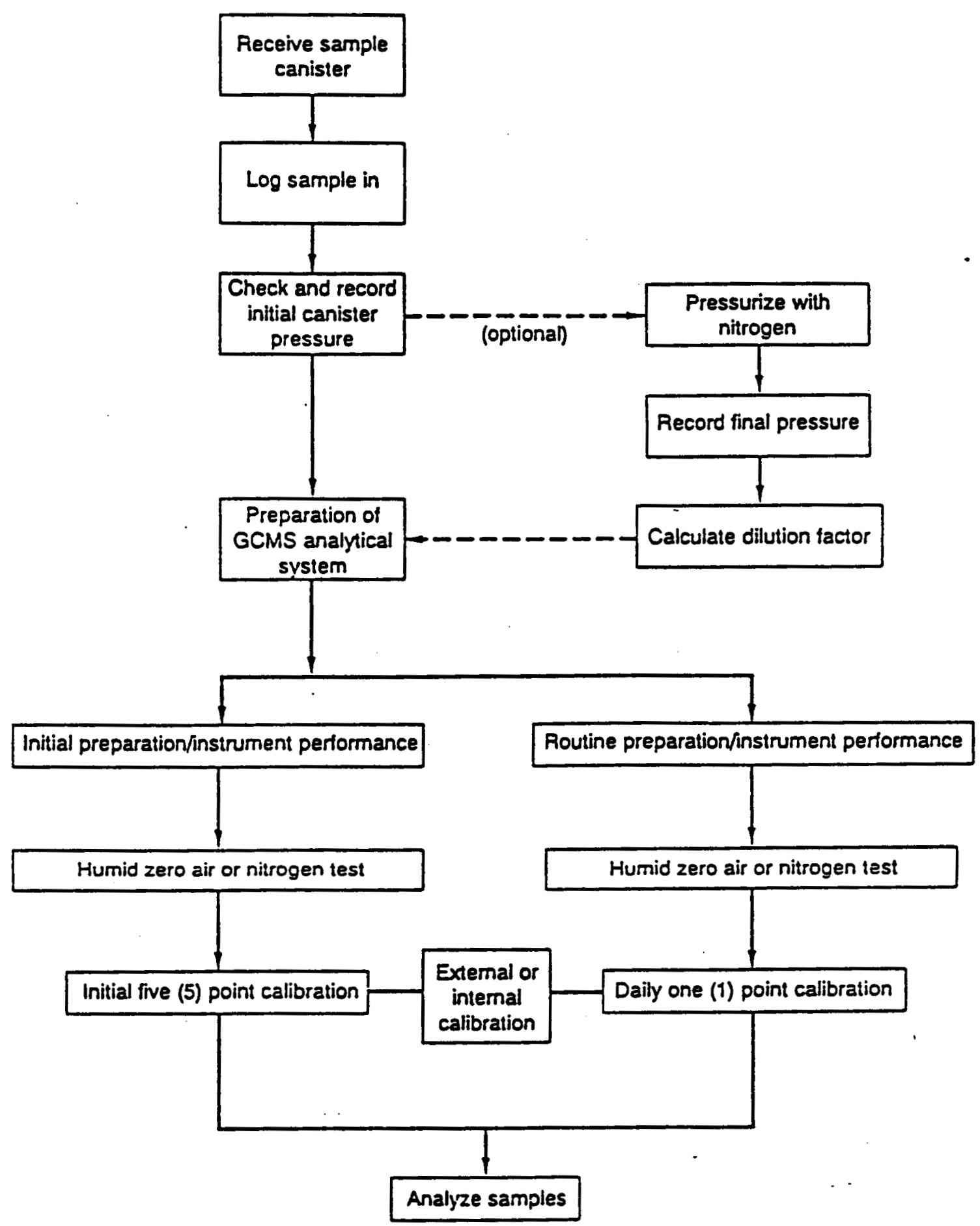

Figure 8. Analytical System Preparation 
7.6.4.4 Measure and document the field canister pressure by closing valve (12) and briefly opening valve (15). Open valve (12) and evacuate to $<0.05 \mathrm{~mm} \mathrm{Hg}$.

7.6.4.5 Pressurize the field canister using zero air or nitrogen by a factor of two. Set three-way valve (40) to vent, set mass-flow controller $(27)$ to $75 \mathrm{~mL} / \mathrm{min}$, and let stabilize for one minute. Close valves (29) and (28), switch three-way valve (40), and open valves (25), (12) and (15). When pressure/vacuum sensor (14) approaches two times the initial pressure measured in 7.6.4.3, close valves (12) and (25) and switch three-way valve (40) to vent. Document the final field canister pressure. The dilution factor is calculated as follows:

$$
D F=\frac{\left(C_{p 2}\right)}{\left(C_{p 1}\right)}
$$

where

$$
\begin{aligned}
& D F=\text { dilution factor } \\
& C_{p 2}=\text { canister pressure after dilution } \\
& C_{p 1}=\text { canister pressure before dilution. }
\end{aligned}
$$

7.6.4.6 Transfer $(50 \times \mathrm{DF}) \mathrm{mL}$ from the field canister to the $500 \mathrm{~mL}$ evacuated laboratory canister (Section 5.3).

7.6.4.7 Final dilution is achieved by delivering $(11500-50) \times \mathrm{DF}] \mathrm{mL}$ of humid zero air or nitrogen to the $500 \mathrm{~mL}$ laboratory canister. Section 5.3 gives details on how to add internal standards prior to the final dilution. A total of $250 \mathrm{ng}$ of each internal standard must be delivered to the analytical system during the sample analysis sequence.

\subsubsection{The final dilution factor is determined by:}

1. Multiplying the dilution factors calculated using equation (6) for steps 7.6.4.4, 7.6.4.5, and 7.6.4.6. The final dilution factor is the product of the individual dilution factors.

2. The ratio of the total gas volume to the gas volume of sample transferred. The volume of sample transferred has to be corrected for any dilutions before the ratio is calculated.

7.6.4.9 The sample is analyzed by attaching the $500 \mathrm{~mL}$ canister to one of the analytical GCMS systems described in Section 7.2. 
7.7 Samples are analyzed by attaching the appropriate sample canister to the inlet of one of the analytical systems described in Section 8.2. If the initial analysis of a sample detects any analytes with concentrations that exceed the initial calibration range, the sample must either be diluted and reanalyzed, or reanalyzed with a lower sample volume.

\subsection{Data Interpretation}

\subsubsection{Qualitative Analysis}

7.8.1.1 An analyte listed in Table 1 is identified by comparison of the sample mass spectrum with the mass spectrum of a standard of the suspected compound (standard reference mass spectrum). Standard reference mass spectra are obtained through analysis of calibration standards. Two criteria must be satisfied to verify identification:

1. A sample component must elute within either the specified GC retention time (RT) or the relative retention time (RRT) window of the analyte in the reference standard.

2. A sample component's mass spectrum must match the mass spectrum of the analyte in the reference standard.

7.8.1.1.1 The RT or RRT of sample components must be determined by comparison with a reference standard analyzed on the same day as the sample. The sample component RRT must compare within \pm 0.06 RRT units of the RRT of the analyte in the reference standard. The RT must compare within plus or minus three standard deviations of the individual retention times of the analyte from the most recent initial calibration. If coelution or interference prohibits accurate assignment of the sample component RT or RRT from the total ion chromatogram, the RT or RRT should be assigned by using the EICP for the primary characteristic ions listed in Table 3.

7.8.1.1.2 All ions present in the standard mass spectrum at a relative intensity greater than $10 \%$ (most abundant ion in the spectrum equals $100 \%$ ) must be present in the sample spectrum. The relative intensities of all ions present at $>10 \%$ must agree to within $\pm 20 \%$ between the standard and sample spectra.

7.8.1.2 For samples containing components not listed in Table 1, with total ion current peak areas greater than $10 \%$ of the nearest (RT) internal standards, a forward library search of the latest NIST mass spectral data base must be performed. With external standard quantitation, unknown components with total ion current peak areas greater than $10 \%$ of the largest target 
compound identified or ten times greater than the standard deviation of the background must be searched. Guidelines for making tentative identification of these unknown compounds are:

1. The relative intensities of major ions in the reference spectrum lions $>10 \%$ of the most abundant ion) should be present in the sample spectrum.

2. The relative intensities of major ions should agree within $\pm 20 \%$.

3. Molecular ions present in the reference spectrum should be present in the sample spectrum.

4. Ions present in the sample spectrum but not in the reference spectrum should be reviewed for possible contamination or presence of coeluting compounds.

Only after visual comparison of the sample spectrum with the results of the library searches will the mass spectral interpretation specialist assign a tentative identification.

\subsubsection{Quantitative Analysis}

7.8.2.1 Once a compound has been qualitatively identified, the quantitation of that compound will be based on the integrated abundance from the EICP of the primary characteristic ion (Table 3). Quantitation will be performed using the internal standard technique or the external standard technique. The internal standard used for quantitation of an analyte must be the same internal standard assigned to the analyte in Table 4.

7.8.2.2 When the analytical system is calibrated in nanograms, the concentration (in ppmv) of each identified analyte in the sample is calculated as follows.

\subsection{Internal standard quantitation}

$$
\text { concentration }(p p m v)=\frac{\left(A_{2}\right)\left(C_{I S}\right)(R)(\eta)}{\left(A_{L S}\right)(R R F)(P)(M W)\left(V_{2}\right)}
$$

where

$A_{x}=$ Area of the characteristic ion for compound being measured

$A_{1 S}=$ Area of the characteristic ion for the internal standard

$C_{1 S}=$ Amount of internal standard, $n g$

RRF = Average relative response factor for compound being measured (Section 7.4) 


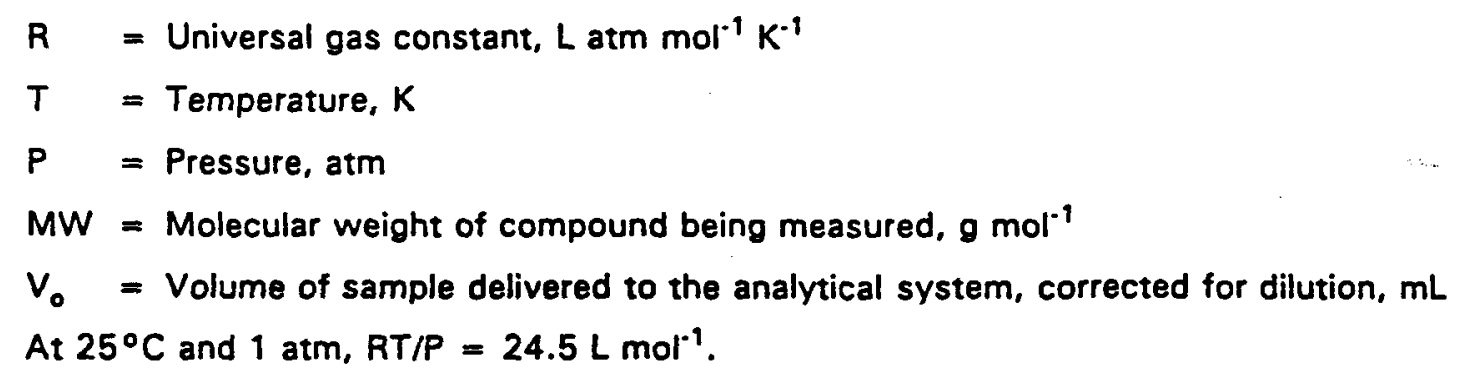

\subsection{External standard quantitation}

$$
\text { concentration (ppmv) }=\frac{\left(A_{2}\right)(R)(I)}{(R F)(P)(M F)\left(V_{0}\right)}
$$

where
$A_{x}, R, T, P, M W$, and $V_{0}$ are the same as for internal standard quantitation.
RF = Average response factor for the compound being measured (Section 7.4).

7.8.2.3 An estimate of the concentration of tentatively identified compounds (TICs) in the sample should be made. The equations given above should be used to determine the estimated concentrations of TICs with the following modifications:

1. The areas $A_{x}$ and $A_{15}$ should be from the total ion chromatograms.

2. The RRF for the TIC is assumed to be one.

3. The nearest internal standard free of interference should be used.

4. The RF for a compound similar in structure should be used.

\subsection{QUALITY CONTROL}

8.1 Each laboratory that uses this procedure is required to operate a formal quality control program. The laboratory must retain records to document the quality of the data generated. Each laboratory must have standard operating procedures documenting and describing activities involved in utilizing this procedure. Specific quality control practices will include, but are not limited to, laboratory duplicates, laboratory blanks, laboratory reference samples, method proficiency samples and blind audit samples (Table 5). Additional information regarding quality assurance/quality control requirements can be found in the WIPP Experimental-Waste Characterization Program QAPP.

8.2 All laboratories using this procedure must demonstrate acceptable performance prior to the analysis of actual samples. Demonstration of acceptable performance will be achieved by analyzing 
Table 5. Scheduled $\mathrm{OC}$ and Calibration

\begin{tabular}{|c|c|c|c|}
\hline $\begin{array}{l}\text { Section } \\
\text { Number }\end{array}$ & Procedure & $\begin{array}{l}\text { Frequency of } \\
\text { OC Procedure }\end{array}$ & Acceptance Criteria \\
\hline 7.3 & BFB Tune & 12 hours & Table 2 \\
\hline 7.4 & 5-pt ICAL & $\begin{array}{l}\text { Initially and } \\
\text { as needed }\end{array}$ & $\begin{array}{l}\text { \%RSD of all compounds }<35 \% \text {; } \\
\text { RRTs within } 0.06\end{array}$ \\
\hline 7.5 & $\begin{array}{l}\text { Continuing } \\
\text { calibration }\end{array}$ & 12 hours & $\begin{array}{l}\% D \text { for all compounds } \\
\text { within } 30 \% \text { of ICAL }\end{array}$ \\
\hline 8.4 & $\begin{array}{l}\text { Laboratory } \\
\text { control } \\
\text { sample }\end{array}$ & $\begin{array}{l}\text { One per analytical } \\
\text { batch or } 5 \% \text { of } \\
\text { all field samples }\end{array}$ & Table 6 \\
\hline 8.5 & $\begin{array}{l}\text { Laboratory } \\
\text { duplicate }\end{array}$ & $\begin{array}{l}5 \% \text { of all } \\
\text { field samples }\end{array}$ & -Table 6 \\
\hline 8.3 & $\begin{array}{l}\text { Laboratory } \\
\text { blank }\end{array}$ & 12 hours & $\begin{array}{l}\text { Target analytes }<50 \% \\
\text { of the PROLs (Table } 6-1 \text { ) }\end{array}$ \\
\hline 8.2 & $\begin{array}{l}\text { Proficiency } \\
\text { testing }\end{array}$ & $\begin{array}{l}\text { Each laboratory, } \\
\text { every six months }\end{array}$ & Table 6 \\
\hline 7.5 & $\begin{array}{l}\text { Internal } \\
\text { standard }\end{array}$ & All samples & $\begin{array}{l}50 \% \text { to } 200 \% \text { of the } \\
\text { average area from the } \\
\text { ICAL and } \pm 30 \text { seconds } \\
\text { retention time from } \\
12 \text {-hour standard }\end{array}$ \\
\hline
\end{tabular}


commercially available (Scott Specialty Gases or equivalent) gas standards or laboratory-prepared gas standards. These standards will contain all of the analytes listed in Table 1 at concentrations appropriate (2-5 times the PROLs) to determine the parameters specified in Table 6 . The analysis of. a minimum of four replicate samples must meet the criteria specified for precision, accuracy and method detection limits in Table 6. Precision will be determined by calculating the percent relative standard deviation of the replicate measurements as follows:

$$
\% R S D=\frac{S}{\bar{C}} \times 100
$$

where

$$
\begin{array}{ll}
\text { \%RSD } & =\text { percent relative standard deviation } \\
\mathbf{S} & =\text { standard deviation } \\
\bar{C} & =\text { mean of replicate analyses. }
\end{array}
$$

Accuracy will be determined as the percent recovery as follows:

$$
\% R=\frac{C_{\mu}}{C_{T}} \times 100
$$

where

$$
\begin{aligned}
& \% R=\text { percent recovery } \\
& C_{M}=\text { measured concentration } \\
& C_{T}=\text { true concentration. }
\end{aligned}
$$

Method detection limit will be determined as follows:

$$
M D L=t_{(n-1,1-2 \cdot 0.20)} \times S
$$

where

$$
\begin{aligned}
& \text { MDL = method detection limit } \\
& \begin{aligned}
S \quad= & \text { standard deviation } \\
\left.t_{(n-1,1-a}=0.89\right)= & t \text {-distribution value appropriate to a } 99 \% \text { confidence level and a standard } \\
& \text { deviation estimate with } n-1 \text { degrees of freedom. }
\end{aligned}
\end{aligned}
$$

Demonstration of acceptable method performance must be repeated at a minimum of every six months. The results of method performance testing must be documented. 
Table 6. VOC Quality Assurance Objectives

\begin{tabular}{lllll}
\hline & $\begin{array}{c}\text { Precision } \\
\text { Compound }\end{array}$ & $\begin{array}{c}\text { Accuracy } \\
\text { (\%RSD or RPD) }\end{array}$ & $\begin{array}{c}\text { MDL } \\
\text { (ng) }\end{array}$ & Completeness \\
\hline $\begin{array}{l}\text { All analytes listed in } \\
\text { Table 1 except: }\end{array}$ & $\pm 25 \%$ & $70-130 \%$ & 8 & $90 \%$ \\
Acetone & $\pm 25 \%$ & $70-130 \%$ & $50^{b}$ & $90 \%$ \\
1-Butanol & $\pm 25 \%$ & $70-130 \%$ & $50^{b}$ & $90 \%$ \\
2 2-Butanone & $\pm 25 \%$ & $70-130 \%$ & $50^{b}$ & $90 \%$ \\
Methanol & $\pm 25 \%$ & $70-130 \%$ & $50^{b}$ & $90 \%$ \\
$4-$ Methyl-2-pentanone & $\pm 25 \%$ & $70-130 \%$ & $50^{b}$ & $90 \%$ \\
\hline
\end{tabular}

- Criteria apply to concentrations listed in Table 1

b Estimated; to be determined

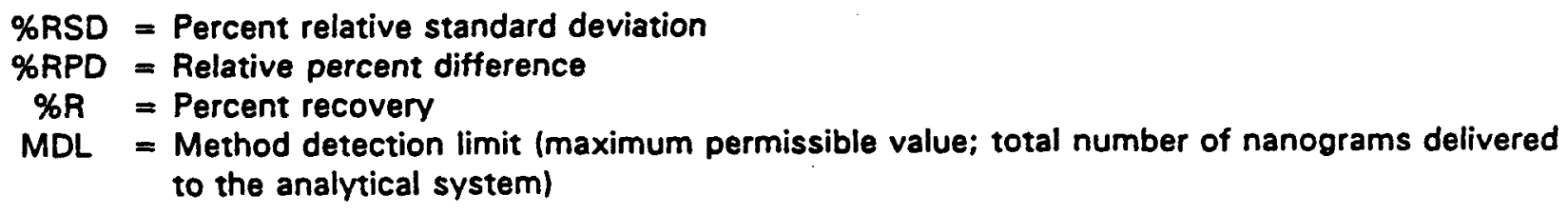


8.3 The laboratory must continually analyze blanks (Table 5). Using the sample preparation manifold, blanks will be generated in the laboratory by filling SUMMA* canisters with humid zero air or nitrogen. These canisters will then be analyzed using the procedures established in Section 7.0. Laboratory blank results are acceptable if the amounts of individual analytes listed in Table 1 are less than $50 \%$ of the PROLs. Laboratory blanks must be analyzed during every 12 -hour shift and prior to the analysis of any samples.

8.4 The laboratory must analyze laboratory control samples (LCSs) on a continuing basis. Commercially purchased gas standards will be used to prepare LCSs. Laboratory control samples will be prepared in the laboratory by attaching the gas standard to the laboratory sample preparation manifold and filling evacuated canisters. The gas standard used to prepare the LCSs must be independent of those used for instrument calibration. These standards must contain some but not all of the analytes listed in Table 1. The concentration of analytes must be in the linear calibration range of the GCMS. Results are acceptable if the criteria specified in Table 6 are met. Laboratory control samples must be analyzed at a frequency of $5 \%$ of all field samples or one per analytical batch, whichever is greater. The results from the analysis of LCSs must be documented.

8.5 The laboratory must analyze individual field samples in duplicate. Field canisters will be analyzed in duplicate at a frequency of $5 \%$ of all field samples or one per analytical batch, whichever is greater. Laboratory duplicate results must meet the criteria specified in Table 6 . The results from the analysis of duplicate samples must be documented.

8.6 The laboratory will also be required to analyze blind audit samples. These audit samples are part of the WIPP Performance Demonstration Program. Details of this program are in the Performance Demonstration Program Plan.

\subsection{Canister Cleaning and Certification}

8.7.1 All canisters must be clean and free of any contaminants ( $<0.5$ times the PROLs) before use.

8.7.2 All canisters must be leak tested by pressurizing them to approximately 30 psig with zero air or nitrogen. The cleaning system in Figure 4 can be used for this task. The initial canister pressure is measured, the valve is closed, and the final pressure is checked after 24 hours. The pressure cannot vary by more than 2 psig over the 24 hour period. 
8.7.3 A canister cleaning system may be assembled as shown in Figure 4 or purchased commercially (Scientific Instrument Specialists, Moscow, ID). The canisters are connected to the manifold and cryogen is added to the trap. The vent shut-off valve and canister valve(s) are opened to release any remaining pressure in the canister(s). The vacuum pump is started and the shut-off valve is opened. If the optional oven is used, the temperature can be set to $150^{\circ} \mathrm{C}$. For canisters with integral vacuum/pressure gauges the maximum temperature is $125^{\circ} \mathrm{C}$ after removing the plastic face plate and rubber blow-out plug. The canisters are evacuated to $<0.05 \mathrm{~mm} \mathrm{Hg}$ for a minimum of one hour. When the optional oven is used, the canisters are evacuated for a minimum of eight hours.

8.7.4 After one hour (or eight hours) of vacuum, the vacuum/pressure gauge shut-off valves are closed and the zero air/nitrogen shut-off valve is opened to pressurize the canisters with zero air or nitrogen at approximately 30 psig.

8.7.5 The zero air/nitrogen shut-off valve is closed and the canister(s) is allowed to vent down to atmospheric pressure through the vent shut-off valve. The vent shut-off valve is then closed. Steps 8.7.3 through 8.7.5 are repeated two additional times for a total of three evacuation/ pressurization cycles for each set of canisters.

8.7.6 The optional oven is turned off, and when canister(s) reach ambient temperature the vacuum/pressure gauge shut-off valves are closed.

8.7.7 At the end of the evacuation/pressurization cycle, the canister (or one canister from the set) is pressurized to $30 \mathrm{psig}$ with humid zero air or nitrogen. The canister is then analyzed by the GCMS analytical system. If no compounds are detected at levels greater than $50 \%$ of the PROLs (Table 1) the canister(s) is clean. If contaminants are found at levels exceeding $50 \%$ of the PROLs, then the cleaning procedure must be repeated until the canister(s) is clean.

8.7.8 The canister is re-attached to the cleaning manifold and evacuated to $<0.05$ $\mathrm{mm} \mathrm{Hg}$. The canister valve is closed and the canister is removed from the cleaning system. The canister remains in this condition until used.

\subsection{GCMS Analytical System Cleaning and Certification}

8.8.1 Cleaning System Components: Components are disassembled and cleaned prior to use. Nonmetallic parts are rinsed with ASTM Type II water and dried in a vacuum oven at $50^{\circ} \mathrm{C}$. 
Stainless steel parts and fittings are cleaned by placing them in a beaker containing methanol and placing the beaker in an ultrasonic bath for 15 minutes. The methanol is replaced with hexane and the ultrasonic process is repeated. The parts and fittings are then rinsed with ASTM Type II water and dried in a vacuum oven at $100^{\circ} \mathrm{C}$.

8.8.2 Humid Zero Air/Nitrogen Test: The sample and calibration preparation manifold (Figure 1 ) is connected to the inlet of the GCMS analytical system, and humid zero air or nitrogen passes through the manifold to the GCMS. The amount of gas delivered to the analytical trap should be the same as that used while analyzing samples (Procedure 430.2). The humid zero air or nitrogen is trapped and subsequently analyzed by GCMS.

In order to pass the humid zero air/nitrogen test, the concentration of the targeted VOCs (Table 1) cannot exceed $50 \%$ of their PROLs.

\subsection{REFERENCES}

1. U.S. Environmental Protection Agency (U.S. EPA), 1988a, Compendium Method TO-14, The Determination of Volatile Organic Compounds (VOC) in Ambient Air Using SUMMA Passivated Canister Sampling and Gas Chromatographic Analyses, May 1988, Quality Assurance Division, Environmental Monitoring Systems Laboratory, Research Triangle Park, North Carolina.

2. U.S. EPA, 1988b, Compendium Method TO-2. The Determination of Volatile Oraanic Compounds in Ambient Air by Carbon Molecular Sieve Adsorption and Gas Chromatography/Mass Spectrometry (GC/MS), Quality Assurance Division, Environmental Monitoring Systems Laboratory, Research Triangle Park, North Carolina.

3. J.H. Myron Stephenson, F. Allen, and T. Slagle, 1990, EPA/Air and Waste Management Association-International Symposium on Measurement of Toxic and Related Air Pollutants.

4. J.A. Glaser, D.L. Foerst, G.D. McKee, S.A. Quave, and W.L. Budde, 1981, Environmental Science and Technology, Vol. 15, No. 12, 1426. 
PROCEDURE 430.2

\section{MODIFIED METHOD 8240/8260 FOR THE \\ DETERMINATION OF VOLATILE ORGANIC COMPOUNDS \\ IN WASTE CONTAINER HEADSPACE COLLECTED USING \\ SUMMA PASSIVATED CANISTERS}

\subsection{SCOPE AND APPLICATION}

1.1 This document describes a procedure for the analysis of the volatile organic compounds (VOCs), listed in Table 1 in concentrations ranging from $1 \mathrm{ppmv}$ (ppm, volume/volume) to volume percent, that have been collected from waste container headspace using SUMMA passivated canisters. This procedure is based on the third addition of U.S. EPA SW-846 Methods 8240/8260, "Gas Chromatography/Mass Spectrometry for Volatile Organics." The sampling procedures are described in the QAPP for the WIPP Experimental-Waste Characterization Program.

1.2 Modifications to Methods $8240 / 8260$ described in this procedure enable the analyst to analyze vaporized VOCs at concentrations $>1$ ppmv in gas samples up to $25 \mathrm{~mL}$ in volume that have been collected using SUMMA passivated canisters. Modifications in this procedure include the use of solid sorbent traps. These traps allow the use of a dry purge cycle to remove water from the solid sorbent column prior to the thermal desorption of trapped VOCs. Solid sorbent trapping is achieved by the use of solid sorbent columns containing only hydrophobic materials lactivated carbon and carbon molecular sieves).

1.3 The procedure described in this document is applicable to the VOCs specified in Table 1. The EPA has not determined the stability of alcohols and ketones when stored in pressurized or subambient pressure SUMMA canisters. It is anticipated that no adverse problems will be encountered with these types of compounds when stored in SUMMA canisters due to the concentration levels at which these compounds are expected to be found.

1.4 This procedure is based upon solid sorbent trapping followed by GCMS analysis and should be restricted to use by or under the supervision of analysts experienced in these areas. In addition, analysts should be skilled in the interpretation of mass spectra and their use as a quantitative tool.

1.5 The program required quantitation limits (PROLs) specified in Table 1 are based on a 10 $\mathrm{mL}$ sample aliquot. PRQLs will be proportionally higher for samples that require dilution or reduced sample size to avoid instrument saturation. 
Table 1. VOC Headspace Target Compound List (TCL) and Program Required Quantitation Limits (PRQLs)

\begin{tabular}{|c|c|c|}
\hline Volatiles & CAS Number & PRQLs (ppmv) \\
\hline $\begin{array}{l}\text { 1. Acetone } \\
\text { 2. Benzene } \\
\text { 3. Bromoform } \\
\text { 4. 1-Butanol } \\
\text { 5. 2-Butanone }\end{array}$ & $\begin{array}{l}67-64-1 \\
71-43-2 \\
75-25-2 \\
71-36-3 \\
78-93-3\end{array}$ & $\begin{array}{c}100 \\
1 \\
1 \\
100 \\
100\end{array}$ \\
\hline $\begin{array}{l}\text { 6. Carbon Tetrachloride } \\
\text { 7. Chlorobenzene } \\
\text { 8. Chloroform } \\
\text { 9. Cyclohexane } \\
\text { 10. 1,1-Dichloroethane }\end{array}$ & $\begin{array}{r}56-23-5 \\
108-90-7 \\
67-66-3 \\
110-82-7 \\
75-34-3\end{array}$ & $\begin{array}{l}1 \\
1 \\
1 \\
1 \\
1\end{array}$ \\
\hline $\begin{array}{l}\text { 11. 1,2-Dichloroethane } \\
\text { 12. 1,1-Dichloroethene } \\
\text { 13. cis-1,2-Dichloroethene } \\
\text { 14. Ethyl Benzene } \\
\text { 15. Ethyl Ether }\end{array}$ & $\begin{array}{r}107-06-2 \\
75-35-4 \\
156-59-2 \\
100-41-4 \\
60-29-7\end{array}$ & $\begin{array}{l}1 \\
1 \\
1 \\
1 \\
1\end{array}$ \\
\hline $\begin{array}{l}\text { 16. Methanol } \\
\text { 17. Methylene Chloride } \\
\text { 18. 4-Methyl-2-pentanone } \\
\text { 19. 1,1,2,2-Tetrachloroethane } \\
\text { 20. Tetrachloroethene }\end{array}$ & $\begin{array}{r}67-56-1 \\
75-09-2 \\
108-10-1 \\
79-34-5 \\
127-18-4\end{array}$ & $\begin{array}{c}100 \\
1 \\
100 \\
1 \\
1\end{array}$ \\
\hline $\begin{array}{l}\text { 21. Toluene } \\
\text { 22. 1,1,1-Trichloroethane } \\
\text { 23. Trichloroethene } \\
\text { 24. 1,1,2-Trichloro-1,2,2-trifluoroethane } \\
\text { 25. 1,3,5-Trimethylbenzene }\end{array}$ & $\begin{array}{r}108-88-3 \\
71-55-6 \\
79-01-6 \\
76-13-1 \\
108-67-8\end{array}$ & $\begin{array}{l}1 \\
1 \\
1 \\
1 \\
1\end{array}$ \\
\hline $\begin{array}{l}\text { 26. 1,2,4-Trimethylbenzene } \\
\text { 27. m-Xylene } \\
\text { 28. o-Xylene } \\
\text { 29. p-Xylene }\end{array}$ & $\begin{array}{r}95-63-6 \\
108-38-3 \\
95-47-6 \\
106-42-3\end{array}$ & $\begin{array}{l}1 \\
1 \\
1 \\
1\end{array}$ \\
\hline
\end{tabular}


1.6 This document only details the sections of Method 8240/8260 that need to be modified to allow the analysis of gas samples. Analysts must refer to Methods $8240 / 8260$ for additional guidance.

\subsection{SUMMARY OF PROCEDURE}

2.1 Gas samples are analyzed by injecting an aliquot of the gas sample into $5 \mathrm{~mL}$ of reagent water that has been spiked with internal standards. A purge-and-trap system transfers any VOCs in the sample to a calibrated GCMS system. Calibration, instrument performance, and data interpretation procedures are identical to those of Methods $8240 / 8260$.

2.2 Upon receipt at the laboratory, a canister containing a sample is attached to the sample manifold (Figure 1). The sample is diluted by direct pressurization with humid zero air or nitrogen and the canister is sampled by removing an aliquot with a gas-tight syringe (Figure 2). The volume of the sample aliquot is determined by multiplying the dilution factor from pressurization times $10 \mathrm{~mL}$. The sample aliquot is then slowly injected into a $25 \mathrm{~mL}$ sparge tube that contains $5 \mathrm{~mL}$ reagent water spiked with internal standards. The VOCs are introduced into the gas chromatograph by the purge-andtrap method. The VOCs are separated on a megabore capillary column and detected using a mass spectrometer operating in the full scan mode. The combination of purge-and-trap introduction with analysis by GCMS allows for the qualitative and quantitative analysis of the VOCs contained in the SUMMA canister. The chromatographic conditions, as well as typical mass spectrometer operating parameters, are given in this method.

2.3 The VOCs in the gas sample form a solution when injected into the $5 \mathrm{~mL}$ of reagent water. An inert gas is bubbled through the resulting solution at ambient temperature, and the VOCs are efficiently transferred (purged) from the solution. The purged VOCs are swept through a sorbent column where the volatile components are trapped. After purging is complete, the sorbent column is heated and backflushed with inert gas to desorb the volatile components onto the head of a megabore capillary column.

\subsection{INTERFERENCES}

3.1 Contamination of samples may occur if the sample canisters are not properly cleaned prior to use. Instructions for cleaning the canisters are described in Section Five of this Guidance Manual. 


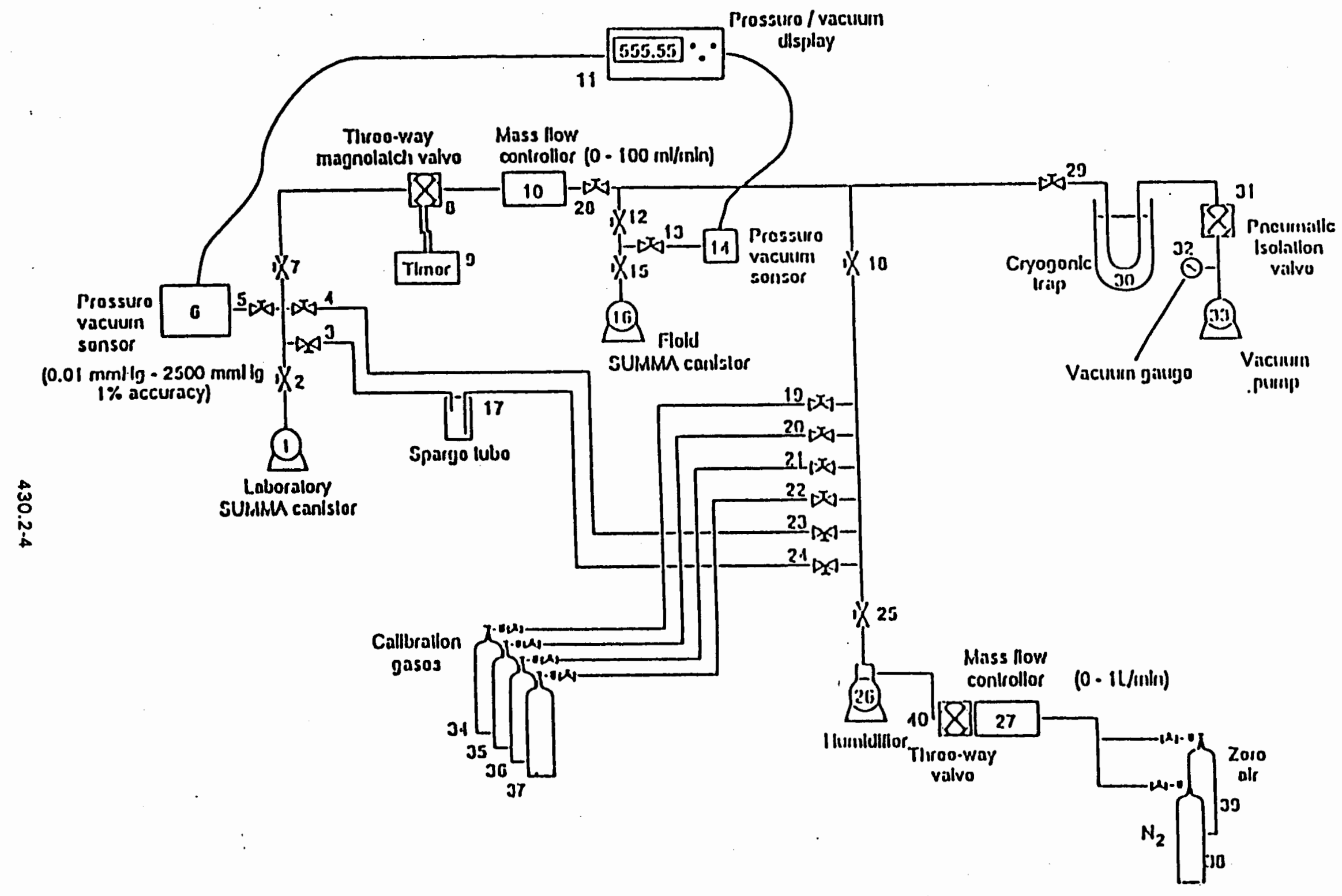

Figure 1. Sample and Calibration Standard Preparation Manifold 


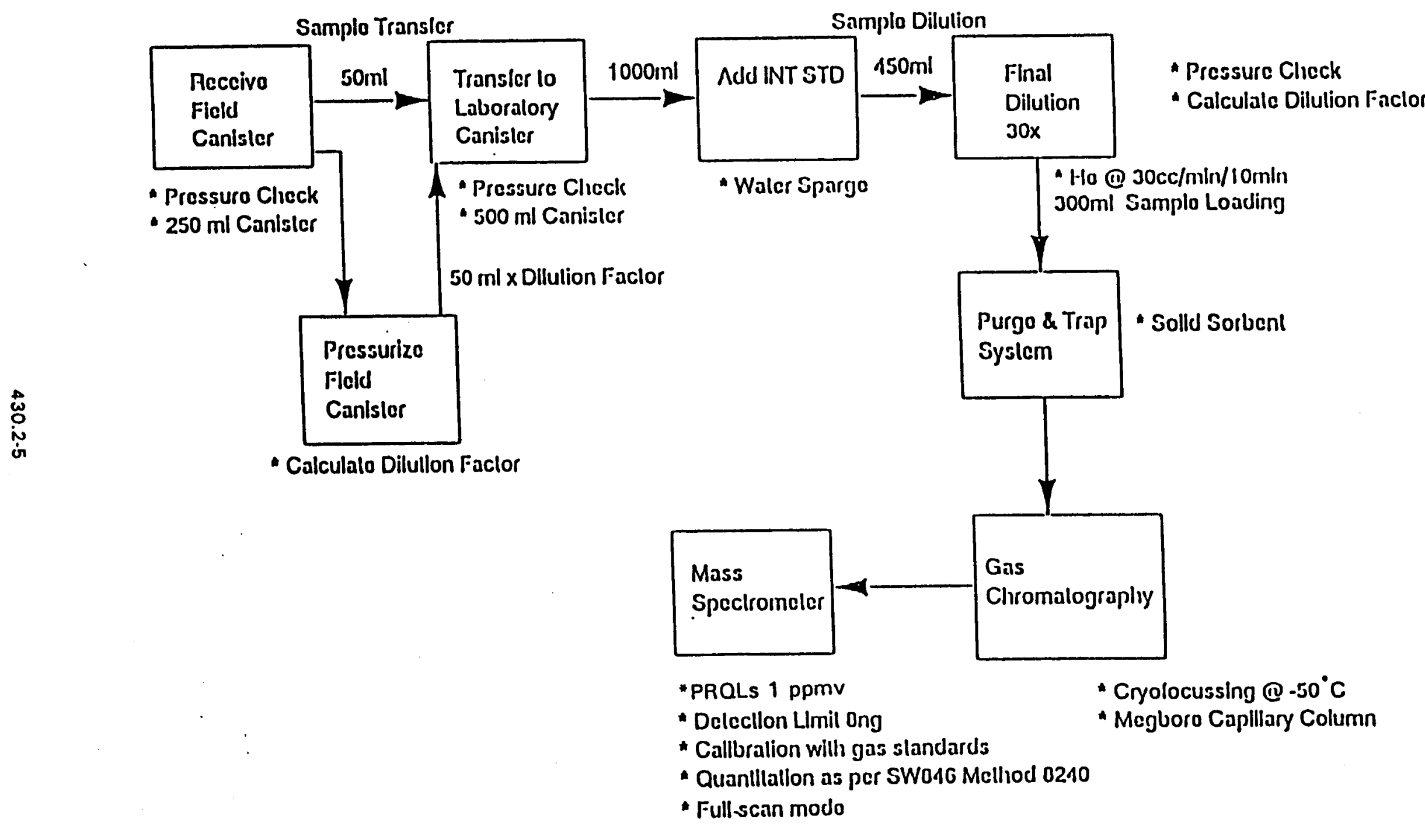

Figure 2. Sample Analysis Flowchart 
3.2 Cross-contamination can occur whenever high-level and low-level samples are analyzed sequentially. Whenever a high-level sample is analyzed, it should be followed by the analysis of ultrapure air or nitrogen.

3.3 For additional information concerning interferences, consult Section 3.0 of Methods $8240 / 8270$.

\subsection{APPARATUS AND MATERIALS}

\subsection{Analytical System}

\subsubsection{Gas Chromatograph/Mass Spectrometer System: Section 4.3 of Method 8260} gives a detailed description of the minimum requirements for the gas chromatograph, the GCMS interface, the gas chromatographic column, the mass spectrometer, and the data system to be used by this method.

\subsubsection{Puroe-and-Trap System: A purge-and-trap device consists of three separate} components: a sparge vessel, a trap, and a desorber. Several complete devices are commercially available. Section 4.1 of Method 8260 and Section 4.11 of Method 8240 present more detailed descriptions of purge-and-trap devices.

\subsubsection{Sparge vessel: A $25 \mathrm{~mL}$ sparge vessel is required by this method.}

4.1.2.2 Trap: The trap must be at least 0.105 inch. The recommended trap should have the following amounts of adsorbents (starting at the trap inlet): $7.6 \mathrm{~cm}$ of Carbopack B and $1.3 \mathrm{~cm}$ of Carbosieve S-III (Supelco, Supelco Park, Bellafonte, PA, Model 2.301 or equivalent.) Before initial use, the trap should be conditioned for one hour at $260^{\circ} \mathrm{C}$ by backflushing with an inert gas flow of at least $30 \mathrm{~mL} / \mathrm{min}$. Vent the trap effluent to a hood, not to the analytical column. Prior to daily use, the trap should be conditioned for 10 minutes at $260^{\circ} \mathrm{C}$ with backflushing.

4.2 Sample and Calibration Standard Preparation Manifold: Section 4.3 of Method Six (Modified Method TO-14) gives a detailed description of the requirements for the sample and calibration standard preparation manifold to be used by this method.

4.3 Syringes: Sections 4.5 through 4.7 of Method 8260 give detailed descriptions of the syringes required by this method. 


\subsection{REAGENTS}

5.1 Propanol, $\mathrm{CH}_{3} \mathrm{CH}_{2} \mathrm{CH}_{2} \mathrm{OH}$ : HPLC grade or equivalent, demonstrated to be free of analytes (less than the PRQLs listed in Table 1).

5.2 Reagent Water: Section 5.6 of Method 8260 describes how to generate interferant-free ASTM Type II water.

5.3 Carrier and Dilution Gases: Helium, nitrogen, and zero air; all ultra-high purity grade.

\subsection{Stock Standards}

5.4.1 Gases: Stock calibration gas standards containing all the analytes listed in Table 1 should be purchased commercially (Scott Specialty Gases or equivalent.) The gas standards should be traceable to a NIST Standard Reference Material (SRM) or to a NIST/EPA approved Certified Reference Material (CRM). All gas standards should be replaced after 12 months.

5.4.2 Liquids: Stock solutions may be prepared from pure standard materials or purchased as certified solutions. Prepare stock solutions in propanol.

5.4.2.1 The preparation of liquid standards is described in Section 5.7 of Method 8260. As an alternative to weighing the flask, the density of the reference material may be used to determine the volume of material to be added to the flask.

5.5 Secondary Dilution Standards: Using stock standard solutions, prepare secondary dilution standards in propanol, containing the compounds of interest, either singly or mixed together. Secondary dilution standards must be prepared fresh every week and stored with minimal headspace.

5.6 Internal Standards: The recommended internal standards are fluorobenzene and chlorobenzene- $d_{5}$. Prepare internal stock standards and secondary dilution standards in propanol if methanol is to be analyzed by this procedure. It is recommended that the secondary dilution internal standard be prepared at a concentration of $25 \mathrm{ng} / \mu \mathrm{L}$ for each internal standard. Addition of $10 \mu \mathrm{L}$ of this standard to $5 \mathrm{~mL}$ of water or calibration standard would be equivalent to $250 \mathrm{ng}$ of each internal standard. 
Revision: 0

Date: June 14, 1991

5.7 4-Bromofluorobenzene (BFB) Standard: A standard solution containing $25 \mathrm{ng} / \mu \mathrm{L}$ of BFB should be prepared.

5.8 Calibration Standards: Calibration standards must be prepared at a minimum of five concentration levels from secondary dilution of stock standards. Prepare these solutions in reagent water. One of the concentrations levels should be at a concentration that delivers an amount (ng) of each analyte equal to a $10 \mathrm{~mL}$ gas sample at the PRQLs listed in Table 1. As an example, a $10 \mathrm{~mL}$ sample of benzene at $1 \mathrm{ppmv}$ contains about $32 \mathrm{ng}$ of benzene at one atmosphere pressure and $25^{\circ} \mathrm{C}$ (Section 5.3.1 of Section Six; modified Method TO-14). The remaining concentration levels should define the linear working range of the GCMS system.

5.9 Laboratory Control Samples (LCSs): LCSs are prepared from stock calibration gas standards in the same way as secondary dilution standards. The preparation of secondary dilution standards is described in Section 5.3 in Section Six (modified Method T0-14).

\subsection{SAMPLE COLLECTION. PRESERVATION AND HANDLING}

6.1 Samples will be collected using the sampling procedures described in the WIPP Experimental-Waste Characterization Program QAPP.

6.2 Sample canisters must be stored at room temperature. Sample holding times cannot exceed 28 days.

6.3 All sample handling and chain-of-custody procedures described in the QAPP for the WIPP Experimental-Waste Characterization Program must be followed.

\subsection{PROCEDURE}

\subsection{Recommended GCMS Operating Conditions}

$\begin{array}{ll}\text { Electron energy: } & 70 \text { volts (nominal) } \\ \text { Mass Range: } & 29-260 \text { amu } \\ \text { Scan Time: } & \text { One second/scan or enough to give } 5 \text { scans/peak } \\ \text { Column type: } & \text { DB-624, 30 } \mathrm{m} \times 0.53 \mathrm{~mm} \text { ID } \\ \text { Initial column temperature: } & 10^{\circ} \mathrm{C} \\ \text { Initial column holding time: } & 5 \text { minutes } \\ \text { Column temperature program: } & 6^{\circ} \mathrm{C} \text { per minute } \\ \text { Final column temperature: } & 160^{\circ} \mathrm{C} \\ \text { Final column holding time: } & \text { Until all compounds have eluted } \\ \text { Injector temperature: } & 200-225^{\circ} \mathrm{C}\end{array}$


Source temperature:

Transfer line temperature:

Carrier gas:
According to manufacturer specifications $250-300^{\circ} \mathrm{C}$

Helium at $15 \mathrm{~mL} / \mathrm{min}$

7.2 GCMS Instrument Performance Testing: Every 12 hours, or prior to a calibration, the GCMS system must meet the BFB criteria specified in Table 2. Instrument performance criteria must be met by putting $50 \mathrm{ng}$ of BFB on column. Instrument hardware tuning may be performed with perfluorotributylamine (PFTBA).

7.3 Purge-and-Trap Assembly: Assemble a purge-and-trap device that meets the specifications given in Section 4.1. Connect the purge-and-trap device to the gas chromatograph of the GCMS system.

7.4 Initial Calibration Procedures: Initially, a five-point calibration is performed on the GCMS system. Each calibration standard must contain all of the analytes in Table 1 and $250 \mathrm{ng}$ of each internal standard. One calibration standard must contain all of the analytes in amounts (ng) equal to a $10 \mathrm{~mL}$ sample near the PRQLs specified in Table 1. Sections 7.2.4 and 7.2.5 of Method 8260 or Sections 7.2.5 and 7.2.6 of Method 8240 give the specific details for performing initial calibrations.

7.4.1 An Extracted Ion Current Profile (EICPI plots ions of a specified mass versus time or scan number. Tabulate the area response of the EICP characteristic ions (Table 3 ) against the amount (ng) for each compound and for each internal standard from the five calibration standards. Calculate the relative response factor (RRF) for each compound using the assignments made in Table 4. When the analytical system is calibrated in nanograms, the RRF is calculated as follows:

$$
R R F=\frac{\left(A_{x} C_{L}\right)}{\left(A_{i s} C_{D}\right)}
$$

where

$$
\begin{aligned}
& A_{x}=\text { area of the characteristic ion for the compound being measured } \\
& A_{1 S}=\text { area of the characteristic ion for the specific internal standard } \\
& C_{1 S}=\text { amount of the specific internal standard, } n g \\
& C_{x}=\text { amount of the compound being measured, } n g .
\end{aligned}
$$

7.4.2 The average RRF must be calculated for each compound. Calculate the percent relative standard deviation (\%RSD) of the RRF from the five calibration points for all compounds listed in Table 1. The \%RSD is calculated as follows: 
Table 2. 4-Bromofluorobenzene Key lons and Abundance Criteria

\begin{tabular}{ll}
\hline Mass & \multicolumn{1}{c}{ Ion Abundance Criteria } \\
\hline 50 & 15 to $40 \%$ of mass 95 \\
75 & 30 to $60 \%$ of mass 95 \\
95 & base peak, $100 \%$ relative abundance \\
96 & 5 to $9 \%$ of mass 95 \\
173 & less than $2 \%$ of mass 174 \\
174 & greater than $50 \%$ of mass 95 \\
175 & 5 to $9 \%$ of mass 174 \\
176 & greater than $95 \%$ but less than $101 \%$ of mass 174 \\
177 & 5 to $9 \%$ of mass 176 \\
\hline \hline
\end{tabular}


Table 4. Volatile Organic Internal Standards With

Corresponding Analytes Assigned for Quantitation

\begin{tabular}{|c|c|}
\hline Fluorobenzene & Chlorobenzene- $d_{5}$ \\
\hline $\begin{array}{l}\text { Acetone } \\
\text { Benzene } \\
\text { 1-Butanol } \\
\text { 2-Butanone } \\
\text { Carbon Tetrachloride } \\
\text { Chloroform } \\
\text { Cyclohexane } \\
\text { 1,1-Dichloroethane } \\
\text { 1,2-Dichloroethane } \\
\text { 1,1-Dichloroethene } \\
\text { cis-1,2-Dichloroethene } \\
\text { Ethyl Ether } \\
\text { Methanol } \\
\text { Methylene Chloride } \\
\text { 1,1,1-Trichloroethane } \\
\text { Trichloroethene } \\
\text { 1,1,2-Trichloro-1,2,2-trifluoroethane }\end{array}$ & $\begin{array}{l}\text { Bromoform } \\
\text { Chlorobenzene } \\
\text { Ethylbenzene } \\
\text { 4-Methyl-2-pentanone } \\
\text { 1,1,2,2-Tetrachloroethane } \\
\text { Tetrachloroethene } \\
\text { Toluene } \\
\text { 1,3,5-Trimethylbenzene } \\
\text { 1,2,4-Trimethylbenzene } \\
\text { m-Xylene } \\
\text { o-Xylene } \\
\text { p-Xylene }\end{array}$ \\
\hline
\end{tabular}




$$
\% R S D=\frac{S}{\bar{X}} \times 100
$$

where

$$
\begin{aligned}
& \bar{X}=\text { mean of } 5 \text { initial RRFs for a compound = average RRF } \\
& S=\text { standard deviation of RRFs for a compound. }
\end{aligned}
$$

The \%RSD for each individual compound must be less than $35 \%$. For those compounds having a \%RSD greater than $35 \%$, the GCMS data system may be used to generate a second or third order regression calibration curve. The initial calibration reporting form (WIPP QAPPI must indicate when higher order calibration curves are utilized. Sample analysis cannot proceed until the \%RSD requirement for each compound is met.

\subsection{Daily GCMS Calibration}

7.5.1 Prior to the analysis of any samples (including standards), instrument performance criteria must be satisfied (Section 7.2).

7.5.2 The initial calibration curve (Section 7.4) for each compound listed in Table 1 must be checked and verified once during every 12-hour shift and prior to the analysis of any samples. This is accomplished by analyzing a calibration standard that contains amounts (ng) of compounds near the midpoint of the initial calibration.

7.5.3 The validity of the initial calibration curve is checked by calculating the percent difference for each compound as follows:

$$
\% D=\frac{\left(R R F_{j}\right)-\left(R R F_{c}\right)}{\left(R R F_{j}\right)}
$$

where

$$
\begin{aligned}
& \% D=\text { percent difference } \\
& R R F_{1}=\text { average relative response factor from initial calibration } \\
& R R F_{C}=\text { relative response factor from current midpoint standard. }
\end{aligned}
$$

The percent difference for all compounds must be less than $30 \%$. For those compounds where a higher order regression curve was used, the point from the continuing calibration standard for the compound must fall within $30 \%$ of the curve value from the initial calibration. If the $\% \mathrm{D}$ criterion is 
not met, corrective action must be taken. If no source of the problem can be determined after corrective action has been taken, a new five-point initial calibration must be generated. This criterion must be met before quantitative sample analysis begins.

7.5.4 The internal standard area responses in the continuing calibration standard must be evaluated immediately after or during sample analysis. If the retention time of any internal standard changes by more than 30 seconds from the last continuing calibration, the chromatographic system must be check for malfunctions and corrections must be made. If the EICP area for any internal standard changes by a factor of two from the last continuing calibration standard, the GCMS system must be checked for malfunctions and corrections must be made. Samples analyzed while the system was malfunctioning must be reanalyzed.

\subsection{Sample Analysis}

7.6.1 The volume of sample required to meet the PROLs specified in Table 1 is $10 \mathrm{~mL}$. This method is designed to analyze gas volumes less than $25 \mathrm{~mL}$.

7.6.2 Pressurize the field canister using zero air or nitrogen by a factor of two. This will facilitate syringe sampling of SUMMA canisters.

\subsubsection{Refer to Sections 7.6.4.2 through 7.6.4.5 of Method Six (Modified} Method TO-14) of this Guidance Manual for details on pressurizing the field canister.

7.6.3 All samples and standard solutions must be allowed to warm to ambient temperature before analysis.

7.6.4 Remove the plunger from a $5-\mathrm{mL}$ syringe and attach a closed syringe valve. Carefully pour reagent water that has been allowed to come to ambient temperature into the syringe barrel to just short of overflowing. Replace the syringe plunger and compress the sample. Open the syringe valve and vent any residual air while adjusting the sample volume to $5.0 \mathrm{~mL}$. Care must be taken to prevent air from leaking into the syringe.

7.6.5 Add $10 \mu$ of internal standard spiking solution through the valve bore of the syringe; then close the valve. The addition of $10 \mu \mathrm{L}$ of the internal standard spiking solution to $5 \mathrm{~mL}$ of sample is equivalent to $250 \mathrm{ng}$ of each internal standard. 
7.6.6 Attach the syringe-syringe valve assembly to the syringe valve on the purging device. Open the syringe valves and inject the sample into the purging chamber.

7.6.7 SUMMA canisters can be sampled with gas-tight syringes by first securing a Teflon-faced septum to the valve inlet using a Swagelok nut.

7.6.7.1 Equip the appropriate Luerlock gas-tight syringe with a syringe valve and needle. Place the syringe plunger into the barrel of the syringe. Open the syringe valve and compress the plunger, forcing the air from the syringe. To flush the syringe, insert the needle of the syringe into the septum of the sample canister. Open the syringe valve and either allow the pressure inside the canister to push the plunger back to volume or pull back on the plunger to fill it to volume. Close the syringe valve and remove the needle from the sample canister. Open the syringe valve and compress the plunger to empty the syringe. If there is adequate sample volume, repeat the process. If not, insert the syringe needle with the syringe valve open into the sample canister and fill the syringe with the desired volume of sample plus $5 \mathrm{~mL}$. Close the syringe valve and remove the needle from the sample canister. Open the syringe valve and compress the plunger to the desired volume and close the syringe valve. Remove the needle from the syringe.

7.6.8 Attach the syringe valve assembly to the syringe valve on the purging device. Open the syringe valves and inject the sample into the purging chamber containing $5 \mathrm{~mL}$ water and $250 \mathrm{ng}$ of each internal standard.

7.6.9 Close both valves, remove the syringe, and purge the sample for a total of 19 minutes $(8$ minutes wet purge and 11 minute dry purge).

7.6.10 At the conclusion of the purge cycle, the trap should be preheated to $100^{\circ} \mathrm{C}$ and the sample desorbed for 4 minutes at $225^{\circ} \mathrm{C}$ while being backflushed with helium.

7.6.10.1 After desorbing the sample for 4 minutes, recondition the trap by baking for 15 minutes at $260^{\circ} \mathrm{C}$. When the trap temperature is less than $30^{\circ} \mathrm{C}$, it is ready for the next sample.

7.6.11 If the initial analysis of a sample detects any analytes with concentrations that exceed the initial calibration range, the sample must be either diluted and reanalyzed or reanalyzed at a lower sample volume. 
7.6.12. When a sample is analyzed that has saturated the detector or shows concentrations of analytes greater than 10 times the upper limit of the initial calibration, the analysis must be followed by a humid zero air blank analysis. If the analysis is not free of interferences (target analytes $<50 \%$ of the PRQLs), the system must be decontaminated. Sample analysis may not resume until a blank can be analyzed that is free of interferences.

\subsection{Data Interpretation}

\subsubsection{Qualitative Analysis}

7.7.1.1 An analyte listed in Table 1 is identified by comparison of the sample mass spectrum with the mass spectrum of a standard of the suspected compound (standard reference mass spectrum). Standard reference mass spectra are obtained through analysis of calibration standards. Two criteria must be satisfied to verify identification:

1. A sample component must elute within either the specified GC retention time (RT) window or the specified relative retention time (RRT) window of the analyte in the standard, and

2. A sample component's mass spectrum must match the mass spectrum of the analyte in the standard.

7.7.1.1.1 The RT or RRT of sample components must be determined by comparison with a reference standard analyzed on the same day as the sample. The sample component RRT must compare within \pm 0.06 RRT units of the RRT of the analyte in the reference standard. The RT must compare within plus or minus three standard deviations of the individual retention times from the most recent initial calibration. If coelution or interference prohibits the accurate assignment of the sample component RT or RRT from the total ion chromatogram, the RT or RRT should be assigned by using the EICP for the primary characteristic ions listed in Table 3.

7.7.1.1.2 All ions present in the standard mass spectrum at a relative intensity greater than $10 \%$ (most abundant ion in the spectrum equals $100 \%$ ) must be present in the sample spectrum. The relative intensities of all ions present at $>10 \%$ must agree to within $\pm 20 \%$ between the standard and sample spectra.

7.7.1.2 For samples containing components not listed in Table 1, with total ion current peak areas greater than $10 \%$ of the nearest (RT) internal standards, a forward library search 
of the latest NIST mass spectral data base must be performed. Guidelines for making tentative identification of these unknown compounds are:

1. The relative intensities of major ions in the reference spectrum lions $>10 \%$ of the most abundant ion) should be present in the sample spectrum.

2. The relative intensities of major ions should agree within $\pm 20 \%$.

3. Molecular ions present in the reference spectrum should be present in the sample spectrum.

4. Ions present in the sample spectrum but not in the reference spectrum should be reviewed for possible contamination or presence of coeluting compounds.

Only after visual comparison of the sample spectrum with the results of the library searches will the mass spectral interpretation specialist assign a tentative identification.

\subsubsection{Quantitative Analysis}

7.7.2.1 Once a compound has been qualitatively identified, the quantitation of that compound will be based on the integrated abundance from the EICP of the primary characteristic ion (Table 3). Quantitation will be performed using the internal standard technique. The internal standard used for the quantitation of an analyte must be the same internal standard assigned to the analyte in Table 4.

7.7.2.2 When the analytical system is calibrated in nanograms, calculate the concentration (in ppmv) of each analyte identified in the sample as follows:

\section{Internal Standard Quantitation:}

$$
\text { concentration (ppmv) }=\frac{\left(A_{\nu}\right)\left(C_{L}\right)(R)(T)}{\left(A_{L S}\right)(R R F)\left(V_{D}\right)(M W)(P)}
$$

where

$$
V_{0}=\left(\frac{V_{0}}{D_{f}}\right)\left(\frac{P_{c}}{760}\right)\left(\frac{298}{273+T_{c}}\right)
$$


and

$$
D_{f}=\frac{C_{P 2}}{C_{P 1}}
$$

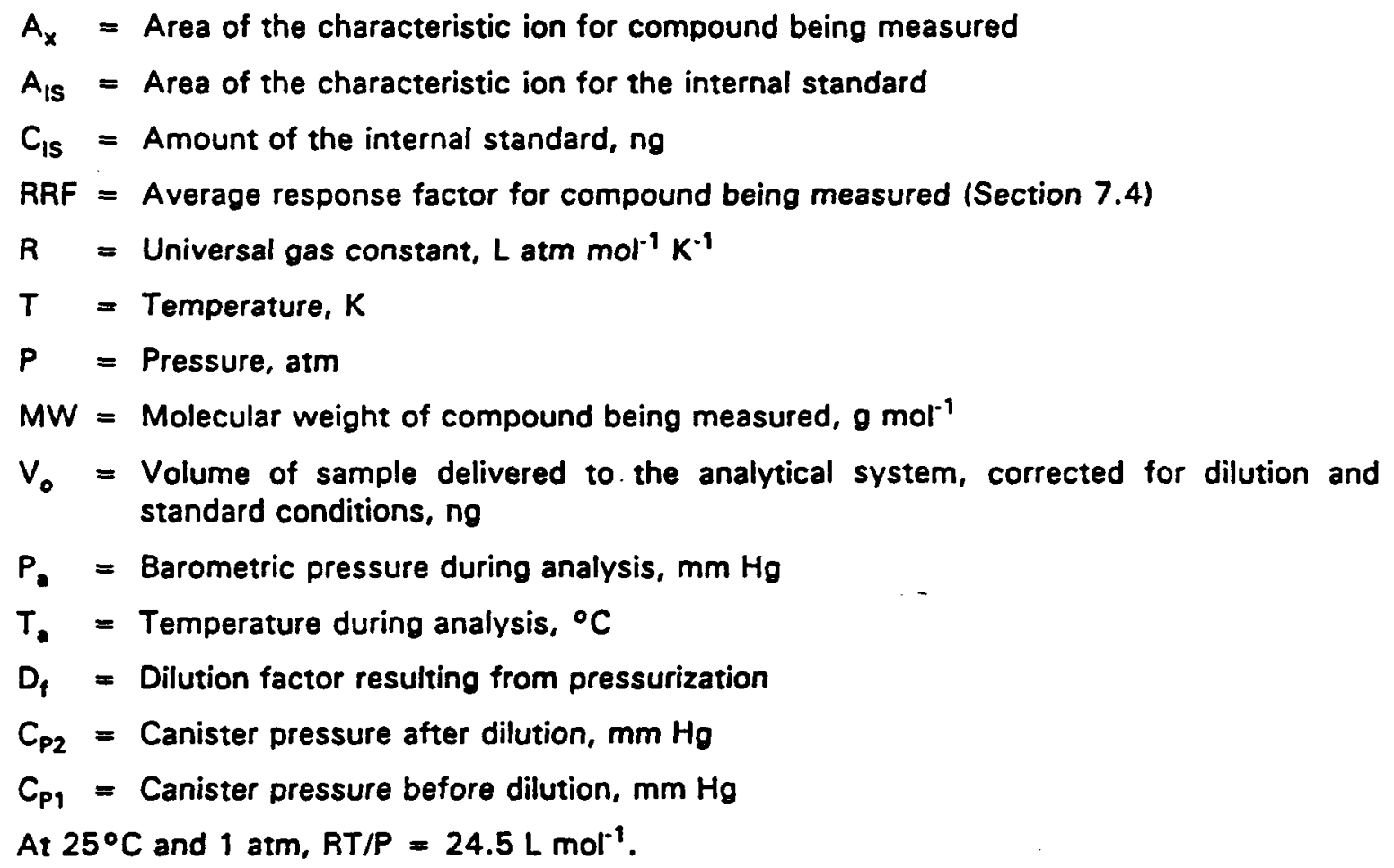

7.7.2.3 An estimate of the concentration of tentatively identified compounds (TICs) in the sample should be made. The equation given above should be used to determine the estimated concentrations of TICs with the following modifications:

1. The areas $A_{x}$ and $A_{1 S}$ should be from the total ion chromatograms.

2. The RRF for the TIC is assumed to be one.

3. The nearest internal standard free of interference should be used.

\subsection{QUALITY CONTROL}

8.1 Each laboratory that uses these methods is required to operate a formal quality control program. The laboratory must retain records to document the quality of the data generated. Each laboratory must have standard operating procedures documenting and describing activities involved in utilizing these methods. Specific quality control practices will include, but are not limited to, laboratory duplicates, laboratory blanks, laboratory reference samples, method proficiency samples and 
blind audit samples (Table 5). Additional information regarding quality assurance/quality control requirements can be found in the WIPP QAPP.

8.2 All laboratories using these methods must demonstrate acceptable performance prior to the analysis of actual samples. Demonstration of acceptable performance will be achieved by analyzing gas standards that contain all of the analytes listed in Table 1 at concentrations appropriate 12-5 times the estimated PRQLs) to determine the parameters specified in Table 6. The analysis of a minimum of four replicate samples must meet the criteria specified for precision, accuracy and method detection limits in Table 6. Precision will be determined by calculating the \%RSD of the replicate measurements as follows:

$$
\% R S D=\frac{S}{\bar{C}} \times 100
$$

where

$$
\begin{array}{ll}
\text { \%RSD } & =\text { percent relative standard deviation } \\
\mathbf{S} & =\text { standard deviation } \\
\overline{\mathbf{C}} & =\text { mean of replicate analyses. }
\end{array}
$$

Accuracy will be determined as the percent recovery as follows:

$$
\% R=\frac{C_{M}}{C_{T}} \times 100
$$

where

$$
\begin{aligned}
& \% R=\text { percent recovery } \\
& C_{M}=\text { measured concentration } \\
& C_{T}=\text { true concentration. }
\end{aligned}
$$

Method detection limit will be determined as follows:

$$
M D L=t_{(n-1.1-c=0.09)} \times S
$$

where

$$
\begin{aligned}
& \text { MOL }=\text { method detection limit } \\
& \mathbf{S}=\text { standard deviation }
\end{aligned}
$$


Table 5. Scheduled $\mathrm{QC}$ and Calibration

\begin{tabular}{|c|c|c|c|}
\hline $\begin{array}{l}\text { Section } \\
\text { Number }\end{array}$ & Procedure & $\begin{array}{l}\text { Frequency of } \\
\text { OC Procedure }\end{array}$ & Acceptance Criteria \\
\hline 7.3 & BFB Tune & 12 hours & Table 2 \\
\hline 7.4 & 5-pt ICAL & $\begin{array}{l}\text { Initially and } \\
\text { as needed }\end{array}$ & $\begin{array}{l}\% \text { RSD all compounds }<35 \% \\
\text { RRTs within } 0.06\end{array}$ \\
\hline 7.5 & $\begin{array}{l}\text { Continuing } \\
\text { calibration }\end{array}$ & 12 hours & $\begin{array}{l}\% D \text { for all compounds } \\
\text { within } 30 \% \text { of ICAL }\end{array}$ \\
\hline 8.4 & $\begin{array}{l}\text { Laboratory } \\
\text { control } \\
\text { sample }\end{array}$ & $\begin{array}{l}\text { One per analytical } \\
\text { batch or } 5 \% \text { of } \\
\text { all field samples }\end{array}$ & Table 6 \\
\hline 8.5 & $\begin{array}{l}\text { Laboratory } \\
\text { duplicate }\end{array}$ & $\begin{array}{l}5 \% \text { of all } \\
\text { field samples }\end{array}$ & Table 6 \\
\hline 8.3 & $\begin{array}{l}\text { Laboratory } \\
\text { blank }\end{array}$ & 12 hours & $\begin{array}{l}\text { Target analytes }<50 \% \\
\text { of the PRQLs (Table } 1 \text { ) }\end{array}$ \\
\hline 8.2 & $\begin{array}{l}\text { Proficiency } \\
\text { testing }\end{array}$ & $\begin{array}{l}\text { Each analyst, } \\
\text { every six months }\end{array}$ & Table 6 \\
\hline 7.5 & $\begin{array}{l}\text { Internal } \\
\text { standard }\end{array}$ & All samples & $\begin{array}{l}50 \% \text { to } 200 \% \text { of } \\
\text { average from ICAL } \\
\text { and } \pm 30 \text { seconds } \\
\text { retention time from } \\
12 \text {-hour standard }\end{array}$ \\
\hline
\end{tabular}


Revision: 0

Date: June 14, 1991

Table 6. VOC Quality Assurance Objectives

\begin{tabular}{lllll}
\hline \hline Compound & $\begin{array}{c}\text { Precision } \\
\text { (\%RSD or RPD) }\end{array}$ & $\begin{array}{c}\text { Accuracy } \\
(\% R)\end{array}$ & $\begin{array}{c}\text { MDL } \\
(\mathrm{ng})\end{array}$ & Completeness \\
\hline $\begin{array}{l}\text { All analytes listed in } \\
\text { Table 1 except: }\end{array}$ & $\pm 25 \%$ & $70-130 \%$ & 8 & $90 \%$ \\
Acetone & $\pm 25 \%$ & $70-130 \%$ & $50^{\mathrm{b}}$ & $90 \%$ \\
$\begin{array}{l}\text { 1-Butanol } \\
\text { 2-Butanone }\end{array}$ & $\pm 25 \%$ & $70-130 \%$ & $300^{\mathrm{b}}$ & $90 \%$ \\
Methanol & $\pm 25 \%$ & $70-130 \%$ & $50^{\mathrm{b}}$ & $90 \%$ \\
4-Methyl-2-pentanone & $\pm 25 \%$ & $70-130 \%$ & $300^{\mathrm{b}}$ & $90 \%$ \\
& $\pm 25 \%$ & $70-130 \%$ & $50^{\mathrm{b}}$ & $90 \%$ \\
\hline
\end{tabular}

- Criteria apply to concentrations listed in Table 1

bstimated; to be determined

\%RSD $=$ Percent relative standard deviation

$\% R P D=$ Relative percent difference

$\% R=$ Percent recovery

MDL = Method detection limit (maximum permissible value; total number of nanograms delivered to the analytical system) 


$$
\begin{aligned}
\left.t_{(n-1,1-a}=0.99\right)= & t \text {-distribution value appropriate to a } 99 \% \text { confidence level and a standard } \\
& \text { deviation estimate with } n-1 \text { degrees of freedom. }
\end{aligned}
$$

Demonstration of acceptable method and analyst performance must be repeated at a minimum of every six months. The results of method performance testing must be documented.

8.3 The laboratory must continually analyze blanks (Table 5). Using the sample preparation manifold, blanks will be generated in the laboratory by filling SUMMA canisters with humid zero air or nitrogen. These canisters will then be analyzed using the procedures established in Section 7.0. Laboratory blank results are acceptable if the amounts of individual analytes listed in Table 1 are less than $50 \%$ of the PROLs. Laboratory blanks must be analyzed during every 12 -hour shift and prior to the analysis of any samples.

8.4 The laboratory must analyze LCSs on a continuing basis. Commercially purchased gas standards will be used to prepare LCSs. LCSs will be prepared in the laboratory by attaching the gas standard to the laboratory sample preparation manifold and filling evacuated canisters. For guidance on preparing LCSs, see Section 5.3 of Method Six (Modified Method T0-14). The gas standard used to prepare the LCSs must be independent of those used for instrument calibration. These standards must contain some but not all of the analytes listed in Table 1. The concentration of analytes must be in the linear calibration range of the GCMS. Results will be acceptable if the criteria specified in Table 6 are met. LCSs must be analyzed at a frequency of $5 \%$ of all field samples or one per analytical batch, whichever is greater. The results from LCSs must be documented.

8.5 The laboratory must analyze individual field samples in duplicate. Field canisters will be analyzed in duplicate at a frequency of $5 \%$ of all field samples or one per analytical batch, whichever is greater. Laboratory duplicate results must meet the criteria specified in Table 6. Duplicate sample results must be documented.

8.6 The laboratory will also be required to analyze blind audit samples. These audit samples are part of the WIPP Performance Demonstration Program. Details of this program are in the WIPP Performance Demonstration Program Plan.

\subsection{REFERENCES}

1. Glaser, J.A., D.L. Foerst, G.D. McKee, S.A. Quave, and W.L. Budde, 1981, Environmental Science and Technology, Vol. 15, No. 12, p. 14261. 
Revision: 0

Date: June 14, 1991

2. U.S. Environmental Protection Agency (U.S. EPA), 1986a, SW-846, Test Methods for Evaluating Solid Waste. Physical/Chemical Methods, Third Edition, Office of Solid Waste and Emergency Response, Washington, D.C.

3. U.S. EPA, 1986b, SW-846, Test Methods for Evaluating Solid Waste, Physical/Chemical Methods, Proposed Update to the Third Edition, Office of Solid Waste and Emergency Response, Washington, D.C. 
PROCEDURE 440.1

\section{GAS CHROMATOGRAPHY-FLAME IONIZATION DETECTOR \\ DETERMINATION OF ALCOHOLS AND KETONES IN \\ WASTE CONTAINER HEADSPACE COLLECTED USING \\ SUMMA PASSIVATED CANISTERS}

\subsection{SCOPE AND APPLICATION}

1.1 This document describes a procedure for the analysis of methanol, butanol, acetone, 2-butanone, and 4-methyl-2-pentanone in the range from $100 \mathrm{ppmv}$ (ppm, volume/volume) to volume percent, in samples that have been collected from waste container headspace using SUMMA ${ }^{\circ}$ passivated canisters. The sampling procedures are described elsewhere in the QAPP for the WIPP Experimental-Waste Characterization Program.

1.2 This procedure may also be used to screen samples for the VOCs listed in Table 2 prior to the analysis of these compounds by GCMS. The screening data may be used to determine appropriate dilution factors for samples prior to analysis by GCMS.

1.3 The EPA has not determined the stability of the alcohols and ketones listed in Table 1 when stored in pressurized or sub-ambient pressure SUMMA canisters. It is anticipated that no problems will be encountered with these compounds when collected and stored in SUMMA canisters due to the concentration levels at which these compounds are to be detected.

1.4 This procedure should be restricted to use by or under the supervision of analysts experienced in gas chromatography.

1.5 This procedure may involve the use of hazardous materials, operations, and equipment and does not purport to address all of the potential safety problems associated with its use. It is the responsibility of whoever uses this procedure to consult and establish appropriate safety and health practices.

\subsection{SUMMARY OF PROCEDURE}

2.1 Gas samples are analyzed for the analytes listed in Table 1 by separating components on two different wide-bore capillary columns followed by detection with flame ionization detectors (FIDs). Samples are introduced to the GC by attaching SUMMA canisters directly to heated gas injection valves with integral sample loops via a heated transfer line. Prior to sample analysis, the SUMMA 
Table 1. GC-FID VOC Headspace Target Compound List (TCL) and Program Required Quantitation Limits (PROLs)

\begin{tabular}{lcc}
\hline \multicolumn{1}{c}{ Volatiles } & CAS Number & PROL (ppmv) \\
\hline 1. Acetone & $67-64-1$ & 100 \\
2. 1-Butanol & $71-36-3$ & 100 \\
3. 2-Butanone & $78-93-3$ & 100 \\
4. Methanol & $67-56-1$ & 100 \\
5. 4-Methyl-2-pentanone & $108-10-1$ & 100 \\
\hline \hline
\end{tabular}


canisters must be pressurized to a minimum of two times ambient atmospheric pressure. This will allow the positive pressure of the sample canister to fill the sample loop. Analyte identification is made by comparison of sample component peak retention times to analyte peak retention times of reference standards analyzed under identical operating conditions (retention time windows). Analyte retention time confirmation must be performed on both analytical columns. Quantitation of a given analyte shall be performed on one of the wide-bore capillary columns by the technique of external quantitation. The dilution factor resulting from pressurization must be used when calculating analyte concentrations. The column used for quantitation must be interferant free in the retention time window corresponding to the analyte. Recommended wide-bore capillary columns and chromatographic conditions are given in Section 4.0.

\subsection{INTERFERENCES}

3.1 Contamination may occur in the analytical system if the canisters are not properly cleaned prior to use. Instructions for cleaning the canisters are given in the "SUMMA Passivated Stainless Steel Certification and Cleaning" procedure in the Guidance Manual.

3.2 Cross-contamination can occur whenever high-level and low-level samples are analyzed sequentially. Whenever a highly concentrated sample is analyzed, it should be followed by the analysis of ultra-high purity air or nitrogen.

3.3 The use of non-Teflon plastic coating, non-Teflon thread sealants, or flow controllers with rubber components in the analytical system should be avoided.

3.4 The analytes listed in Table 2 may be present in some of the samples. The chromatographic conditions employed must minimize any potential interference of Table 2 analytes on the target analytes listed in Table 1.

\subsection{APPARATUS AND MATERIAL}

4.1 Gas Chromatograph Analytical System: Complete analytical system to meet the requirements of the WIPP Experimental-Waste Characterization Program QAPP.

4.1.1 Sample Introduction System: System must be capable of attaching SUMMA canisters directly to the gas chromatograph inlet. The introduction system must be capable of heating and maintaining the SUMMA canisters and transfer line to the $\mathrm{GC}$ inlet at $100^{\circ} \mathrm{C}$. The introduction 
Table 2. Potential Voc Interferant Compounds

\begin{tabular}{|c|c|c|}
\hline & Compound & CAS Number \\
\hline $\begin{array}{l}1 . \\
2 . \\
3 . \\
4 . \\
5 .\end{array}$ & $\begin{array}{l}\text { Benzene } \\
\text { Bromoform } \\
\text { Carbon Tetrachloride } \\
\text { Chlorobenzene } \\
\text { Chloroform }\end{array}$ & $\begin{array}{r}67-64-1 \\
75-25-2 \\
56-23-5 \\
108-90-7 \\
67-66-3\end{array}$ \\
\hline $\begin{array}{l}6 . \\
7 . \\
8 . \\
9 . \\
10 .\end{array}$ & $\begin{array}{l}\text { Cyclohexane } \\
\text { 1,1-Dichloroethane } \\
\text { 1,2-Dichloroethane } \\
\text { 1,1-Dichloroethene } \\
\text { cis-1,2-Dichloroethene }\end{array}$ & $\begin{array}{r}110-82-7 \\
75-34-3 \\
107-06-02 \\
75-35-4 \\
156-59-2\end{array}$ \\
\hline $\begin{array}{l}11 . \\
12 . \\
13 . \\
14 . \\
15 .\end{array}$ & $\begin{array}{l}\text { Ethyl Benzene } \\
\text { Ethyl Ether } \\
\text { Methylene Chloride } \\
\text { 1,1,2,2-Tetrachloroethane } \\
\text { Tetrachloroethene }\end{array}$ & $\begin{array}{r}100-41-4 \\
60-29-7 \\
75-09-2 \\
79-34-5 \\
127-18-4\end{array}$ \\
\hline $\begin{array}{l}16 . \\
17 . \\
18 . \\
19 . \\
20 .\end{array}$ & $\begin{array}{l}\text { Toluene } \\
\text { 1,1,1-Trichloroethane } \\
\text { Trichloroethene } \\
\text { 1,1,2-Trichloro-1,2,2-trifluoroethane } \\
\text { 1,3,5-Trimethylbenzene }\end{array}$ & $\begin{array}{r}108-88-3 \\
71-55-6 \\
79-01-6 \\
76-13-1 \\
108-67-8\end{array}$ \\
\hline $\begin{array}{l}21 . \\
22 . \\
23 . \\
24 .\end{array}$ & $\begin{array}{l}\text { 1,2,4-Trimethylbenzene } \\
\text { m-Xylene } \\
\text { o-Xylene } \\
\text { p-Xylene }\end{array}$ & $\begin{array}{r}95-63-6 \\
108-38-3 \\
95-47-6 \\
106-42-3\end{array}$ \\
\hline
\end{tabular}


system must be constructed of materials that minimize adsorption of sample components and minimize the loss of sample due to excessive transfer line volume.

\subsubsection{GC Sample Inlet System: GC sample inlet system must consist of a gas injection} valve with integral fixed sample loop (Valco Instruments EC6UWE or equivalent). The gas injection valve and fixed volume sample loop must be capable of being heated to $100^{\circ} \mathrm{C}$ and must be constructed of materials that minimize adsorption of sample components. The sample injection valves may be configured such that the sample is swept by carrier gas directly onto the GC column or into a split/splitless injection port. Two gas injection valves with fixed sample loops may be connected in series or parallel to enable simultaneous injection on two different capillary columns.

\subsubsection{Oven Temperature Control: The GC oven must be capable of sub-ambient} temperature programming and temperature programming with multiple temperature ramps in one program method. Isothermal temperature control to $\pm 0.5^{\circ} \mathrm{C}$ is recommended.

4.1.4 Detector: The detector must be a flame ionization detector with sufficient sensitivity to meet the quality assurance objectives listed in Table 3.

4.1.5 Carrier Gas Flow: The system must have the capability to regulate the flow of carrier gas through the gas injection valve and analytical capillary column, regulate split flows, and provide make-up gas for the detector.

4.1.6 Analvtical Columns: Wide-bore $(\geq 0.32 \mathrm{~mm})$ capillary columns are required. The recommended columns are 30-meter DB-624 and DB-1701 megabore columns (J\&W Scientific or equivalent).

4.1.7 Data System: The chromatographic system must have a data system that receives and displays signal output from the GC-FID for the entire chromatographic program. The data system must be capable of reprocessing data, external standard calculations, and archiving data for future processing.

\subsection{Gas Standard Preparation Equipment}

4.2.1 Syringes: $10-\mu \mathrm{L}, 25-\mu \mathrm{L}, 100-\mu \mathrm{L}, 250-\mu \mathrm{L}$, and 500- $\mu \mathrm{L}$ microsyringes with 20-gauge needle; $1-\mathrm{mL}, 5-\mathrm{mL}, 10-\mathrm{mL}$, and $25-\mathrm{mL}$ gas tight syringes with shut-off valves. 
Table 3. Quality Assurance Objectives

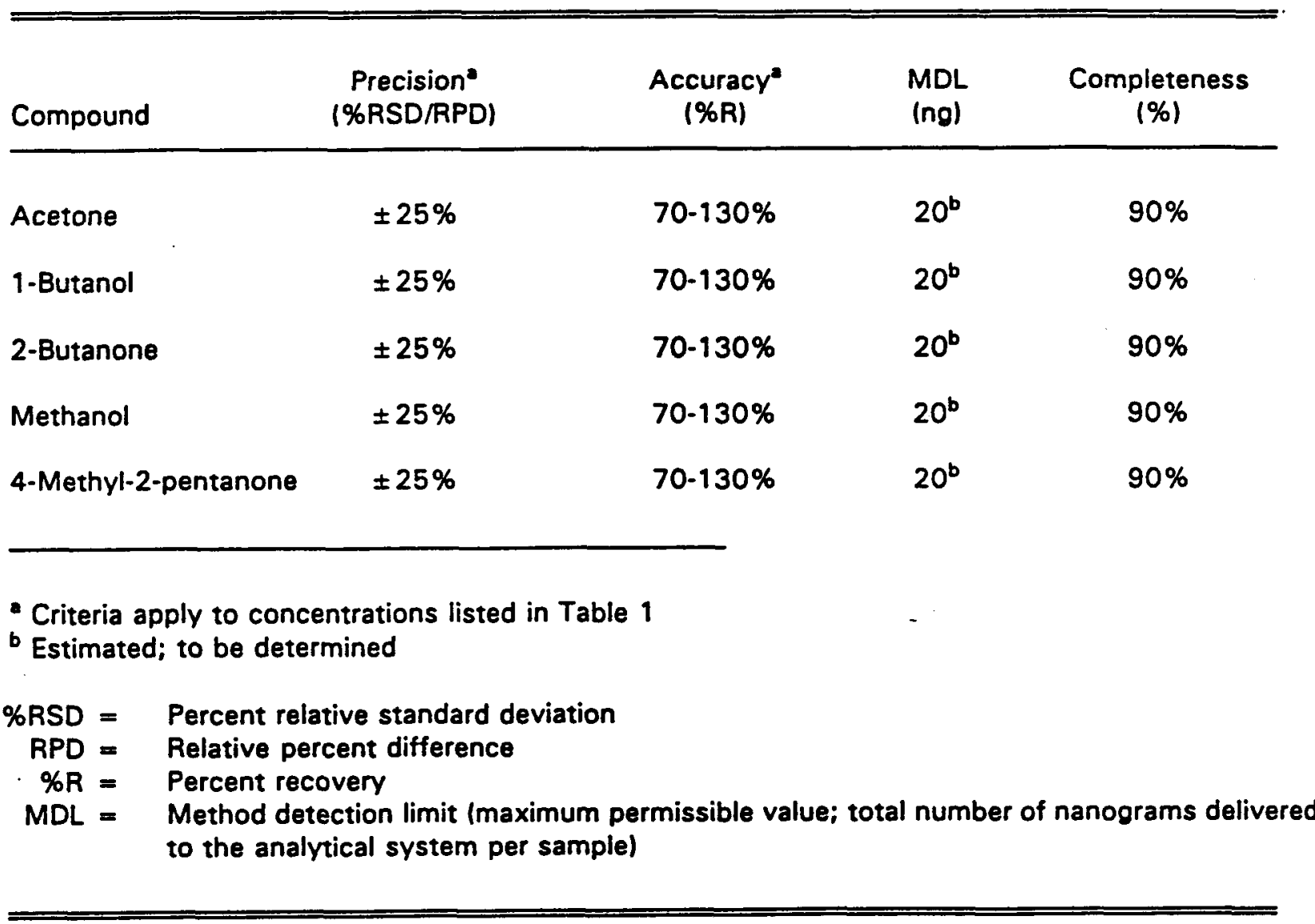


4.2.2 Static Dilution Bottle: Static dilution bottle (glass) with shut-off valve and septum (Tekmar Company or equivalent).

4.2.3 Canisters: SUMMA passivated stainless steel canisters (Scientific Instrument Specialist or equivalent).

\subsection{REAGENTS}

5.1 Carrier and Dilution Gases: Gas cylinders of helium, nitrogen, and zero air; all ultra-high purity grade.

\subsection{Stock Standards}

5.2.1 Gases: Stock gas calibration cylinder(s) of all analyes listed in Table 1 may be purchased commercially(Scott Specialty Gas or equivalent). The standards should be traceable to NIST Standard Reference Material (SRM) or to a NIST/EPA approved Certified Reference Material (CRM). All standards must be replaced after 12 months if comparison with a check standard indicates a problem.

5.2.2 Liquids: Stock solutions of Table 1 analytes may be prepared from pure standard materials or purchased as certified solutions. Solutions may be prepared neat or in an appropriate solvent.

5.2.2.1 Stock solutions should be prepared in $10 \mathrm{~mL}$ volumetric flasks. Solutions may be prepared by weighing or transferring known volumes using microliter syringes. If known volumes are used, the density of the pure material is used to determine the final concentration.

5.2.2.2 Stock standard should be stored in Teflon-sealed screw cap bottles. Store with minimum headspace at $-10^{\circ} \mathrm{C}$ to $20^{\circ} \mathrm{C}$ and protect from direct sunlight.

5.2.2.3 All standards must be replaced at a minimum of every six months or sooner if comparison with check standards indicates a problem. 


\subsection{Secondary Dilution Standards}

5.3.1 Using the stock calibration gas standards, prepare secondary dilution standards containing all the analytes in Table 1 in SUMMA canisters. Secondary dilution standards prepared in SUMMA ${ }^{*}$ canisters must be humidified to a minimum of $20 \%$ relative humidity. Humid secondary dilution standards may be prepared by either directly injecting reagent water (ASTM Type II or equivalent) into an evacuated SUMMA canister or by passing the dilution gas (air or $\mathrm{N}_{2}$ ) through a humidifier.

5.3.2 Secondary dilution standards containing all the analytes listed in Table 1 may be prepared directly in dilution bottles or evacuated SUMMA canisters by injecting aliquots of neat standards or solutions prepared in an appropriate solvent. Secondary dilution standards prepared in SUMMA canisters must be humidified to a minimum of $20 \%$ relative humidity.

5.3.2.1 The amount of each standard to be injected into the dilution bottle or SUMMA canister can be calculated from the desired injection quantity and volume using the following equation:

$$
W_{T}=\frac{W_{l}}{V_{l}} \times V_{s}
$$

where

$W_{T}=$ total quantity of analyte to be injected into the dilution bottle/canister, $\mathrm{mg}$

$W_{1}=$ desired amount of analyte to be injected onto the GC column, $\mathrm{ng}$

$V_{B}=$ total volume of the dilution bottle or the total gas volume in the SUMMA ${ }^{\star}$ canister after pressurization, $\mathrm{mL}$

$V_{1}=$ volume of sample delivered to the $\mathrm{GC}$ column, $\mu \mathrm{L}$.

5.3.2.2 The volume of the neat standard to be injected into the dilution bottle or SUMMA canister is calculated as follows:

$$
V_{T}=\frac{W_{T}}{d}
$$


where

$V_{T}=$ total volume of neat liquid or solution to be injected, $\mu \mathrm{L}$

$d=$ density of the neat standard or the concentration of the stock solution, $\mathrm{g} \mathrm{mL}^{-1}$.

5.3.3 After secondary dilution standards are prepared, they must be allowed to equilibrate for 24 hours prior to use.

5.3.4 Calibration Standards: Calibration standards must be prepared at a minimum of three concentration levels according to the procedures described above. One of the calibration standards must be prepared near the PROLs specified in Table 1. The remaining standards must define the linear working range of the GC system. Each calibration standard must contain all of the analytes listed in Table 1.

\subsection{SAMPLE COLLECTION, PRESERVATION AND HANDLING}

6.1 Samples will be collected according to the sampling procedures described in the WIPP Experimental-Waste Characterization Program QAPP.

6.2 Sample canisters will be stored at room temperature. Sample holding times cannot exceed 28 days.

6.3 All sample handling and chain-of-custody procedures described in the QAPP for the WIPP Experimental-Waste Characterization Program must be followed.

\subsection{PROCEDURE}

\subsection{Recommended GC Analytical System Operating Conditions}

7.1.1 Samole Introduction System: Sample canisters and calibration standard canisters should be maintained at $65^{\circ} \mathrm{C}$ for 30 minutes prior to sample analysis and during sample transfer to the GC. The sample transfer line to the GC should be maintained at a-minimum of $10^{\circ} \mathrm{C}$ above the sample canister temperature. The transfer line should be purged with carrier gas between samples.

7.1.2 GC Sample Inler: The gas injection valve and fixed volume sample loop should be maintained at the same temperature as the sample canisters. The minimum sample loop volume should be $0.5 \mathrm{~mL}$. 
7.1.3 Column Types: DB-62430m $\times 0.53 \mathrm{~mm}$ ID and DB-1701 $30 \mathrm{~m} \times 0.53 \mathrm{~mm} 1 \mathrm{D}$ (J\&W Scientific or equivalent).

7.1.4 Temperature Program (both columns): Initial temperature $10^{\circ} \mathrm{C}$ for 5 minutes, program at $1.5^{\circ} \mathrm{C}$ per minute to $20^{\circ} \mathrm{C}$, then $3.0^{\circ} \mathrm{C}$ per minute to $50^{\circ} \mathrm{C}$, then $5.0^{\circ} \mathrm{C}$ per minute to $150^{\circ} \mathrm{C}$ until all compounds have eluted.

7.1.5 Carrier Gas: Helium at $8 \mathrm{~mL} / \mathrm{min}$, detector make-up gas helium at $22 \mathrm{~mL} / \mathrm{min}$.

\subsubsection{Injector Temperature: $175-225^{\circ} \mathrm{C}$.}

7.1.7 FID Detector System: The FID burner air, hydrogen, and helium carrier flow rates should be set to manufacturers' instructions to maintain as stable a flame as possible through the program cycle.

\subsection{External Standard Calibration}

7.2.1 Initial Calibration Procedure: Initially a three-point calibration is performed on the GC analytical system. Each calibration standard must contain all of the analytes in Table 1. One calibration standard must be near the PROLs listed in Table 1.

7.2.1.1 Inject each calibration standard using a gas injection valve with a fixed volume sample loop. All standards and samples must be analyzed under the same operating conditions. Tabulate peak area response of analyte against the amount injected. Calculate a response factor (RF) for each compound as follows:

$$
R F=\frac{A_{x}}{C_{x}}
$$

where

$$
\begin{aligned}
& A_{x}=\text { total area response for the compound } \\
& C_{x}=\text { amount of the compound being measured, } n g .
\end{aligned}
$$

RF must be determined for all compounds on both analytical columns. 
7.2.1.2 Using the RFs, the percent relative standard deviation (\%RSD) for each compound listed in Table 1 is calculated as follows:

$$
\% R S D=\frac{s}{\bar{X}} \times 100
$$

where

$$
\begin{aligned}
& \bar{X}=\text { mean of the } 3 \text { initial RFs for a compound } \\
& s \quad=\text { standard deviation of the RFs for a compound. }
\end{aligned}
$$

The \%RSD for each individual compound must be less than $30 \%$. For those compounds having a \%RSD greater than $30 \%$, the GC data system may be used to generate a second or third order regression curve. Sample analysis must not proceed until the \%RSD requirement is satisfied.

7.2.2 Calibration Verification Procedure: The working calibration curve or response factor and the retention time window (Section 7.3) must be verified every 12 hours of operation, and prior to the analysis of any samples. Verification is accomplished by analyzing a calibration standard that contains amounts (ng) of each target analyte that are near the midpoint amounts of the initial calibration curve.

7.2.2.1 The verification procedure involves calculating the RF for each compound in the calibration verification standard and comparing these to the average RFs for each compound from the initial calibration sequence. The validity of the initial calibration RF is checked by calculating the percent difference (\%D) for individual compounds as follows:

$$
\% D=\frac{\left(R F_{\eta}\right)-\left(R F_{d}\right)}{\left(R F_{\eta}\right)} \times 100
$$

where

$$
\begin{aligned}
& \% D=\text { percent difference } \\
& R F_{1}=\text { average } R F \text { from initial calibration } \\
& R F_{C}=\text { response factor from the current midpoint calibration verification standard. }
\end{aligned}
$$

The percent difference for all compounds must be less than $30 \%$. For compounds where a higher order regression calibration curve was used, the point from the calibration verification standard for the 
compound must fall within $30 \%$ of the curve value from the initial calibration. If the $\% \mathrm{D}$ criterion is not met, corrective action must be taken. If no source of the problem can be determined after corrective action has been taken, a new three-point initial calibration curve must be generated. This criterion must be satisfied before sample analysis begins.

7.2.2.2 The absolute retention time of each component must fall within the retention time window (Section 7.3) established for that compound during the most recent initial calibration. If the retention time window criterion is not met, corrective action must be taken. If no source of the problem can be determined after corrective action has been taken, a new three-point initial calibration curve must be generated.

\subsection{Retention Time Windows}

7.3.1 Retention time windows for each compound are determined by calculating the standard deviation of the absolute retention time for each compound over the three initial calibration standards. The retention time window shall be defined as plus-or-minus three times the standard deviation of the absolute retention time of each compound determined from the most recent initial calibration.

7.3.2 Retention time windows for each compound must be determined whenever a new initial calibration curve is generated or when a new GC column is installed. Retention time windows must be established for all compounds in Table 1 for both analytical columns.

7.3.3 For the calibration verification standard, the midpoint of the retention time window shall be the average absolute retention time for each compound from the most recent initial calibration.

7.3.4 The midpoint of the 12-hour retention time window shall be the absolute retention time for each analyte from the calibration verification standard for that calibration period.

\subsection{Sample Analysis}

7.4.1 The minimum sample volume required meet the PROLs specified in Table 1 is $0.5 \mathrm{~mL}$. 
7.4.2 All samples must be pressurized to a minimum of two times atmospheric pressure. Samples should be pressurized with humid zero air or nitrogen. The resulting dilution factor is calculated as follows:

$$
D F=\frac{C_{p 2}}{C_{p l}}
$$

where

$$
\begin{aligned}
& D F=\text { dilution factor } \\
& C_{p 2}=\text { canister pressure after dilution } \\
& C_{p 1}=\text { canister pressure before dilution. }
\end{aligned}
$$

After pressurization, samples must be allowed to equilibrate overnight before sample analysis.

7.4.3 Samples are attached to the sample introduction system and are allowed to equilibrate at a minimum of $65^{\circ} \mathrm{C}$ for at least 30 minutes.

7.4.4 After reaching thermal equilibrium, sample is allowed to flow through the sample introduction system and sweep through the fixed volume sample loop. A minimum of 10 fixed loop volume equivalents should be allowed to sweep through the sample loop. A flow restriction device (mass flow controller or mass flow meter) should be placed on the outlet of the sample loop.

7.4.5 After the fixed volume sample loop has been conditioned, the gas injection valve should be rotated to the inject position. The GC temperature program must be started at this time. The gas injection valve should have an electronic event module that automatically starts the GC upon sample injection.

7.4.6 If the initial analysis of a sample detects any analytes with concentrations that exceed the initial calibration range, the sample must be diluted and reanalyzed.

7.4.7 When a sample has been analyzed that saturates the detector or shows concentrations of analytes greater than ten times the upper limit of the initial calibration, the analysis must be followed by a humid zero air or nitrogen blank analysis. If the blank analysis is not free of interferences (target analytes $<50 \%$ of the PROLs) the system must be decontaminated. Sample analysis may not resume until a blank can be analyzed that is free of contamination. 


\subsection{Data Interpretation}

\subsubsection{Qualitative Analysis}

7.5.1.1 An analyte listed in Table 1 is identified by retention time confirmation on both analytical columns.

7.5.1.2 For positive analyte identification, the sample component peak must fall within the retention time window set by the most recent continuing calibration for each analytical column.

\subsubsection{Quantitative Analysis}

7.5.2.1 Once a compound has been qualitatively identified, the quantitation of that compound will be based on the area response. Quantitation of a given compound shall be performed on one of the two analytical columns. The column used for quantitation should be interferant free in the retention time window corresponding to the analyte.

7.5.2.2 Calculate the concentration in ppmv of each identified analyte in the sample as follows:

$$
\text { concentration (ppmv) }=\frac{\left(A_{x}\right)(R)(T)}{(R F)(P)(M W)\left(V_{\nu}\right)}
$$

where

$$
\begin{aligned}
& A_{x}=\text { Total area response for the compound being measured } \\
& R F=\text { Average response factor from most recent initial calibration (Section 7.2) } \\
& R \quad=\text { Universal gas constant, } \mathrm{L} \text { atm mol }{ }^{-1} \mathrm{~K}^{-1} \\
& T \quad=\text { Temperature, } \mathrm{K} \\
& \mathrm{P} \quad=\text { Pressure, atm } \\
& \mathrm{MW}=\text { Molecular weight of compound being measured, } \mathrm{g} \mathrm{mol}{ }^{-1} \\
& \mathrm{~V}_{0} \quad=\text { Volume of sample delivered to the analytical system, corrected for dilution, } \mathrm{mL} \\
& \text { At } 25^{\circ} \mathrm{C} \text { and } 1 \mathrm{~atm}, \mathrm{RT} / \mathrm{P}=24.5 \mathrm{~L} \mathrm{~mol}^{-1} \text {. }
\end{aligned}
$$




\subsection{QUALITY CONTROL}

8.1 Each laboratory that uses this procedure is required to operate a formal quality control program. The laboratory must retain records to document the quality of the data generated. Each laboratory must have standard operating procedures documenting and describing activities involved in utilizing this procedure. Specific quality control practices will include, but are not limited to, laboratory duplicates, laboratory blanks, laboratory reference samples, method proficiency samples and blind audit samples (Table 4). Additional information regarding quality assurance/quality control requirements can be found in the WIPP Experimental-Waste Characterization Program QAPP.

8.2 All laboratories using this procedure must demonstrate acceptable performance prior to the analysis of any samples. Demonstration of acceptable performance will be achieved by analyzing commercially available (Scott Specialty Gases or equivalent) gas standards or laboratory prepared gas standards. These standards will contain all of the analytes listed in Table 1 at concentrations appropriate (two to five times the estimated MDLs) to determine the parameters specified in Table 3 . The analysis of a minimum of four replicate samples must meet the criteria specified for precision, accuracy and method detection limits in Table 3. Precision will be determined by calculating the percent relative standard deviation of the replicate measurements as follows:

$$
\% R S D=\frac{S}{\bar{C}} \times 100
$$

where

$$
\begin{array}{ll}
\text { \%RSD } & =\text { percent relative standard deviation } \\
\mathrm{S} & =\text { standard deviation } \\
\bar{C} & =\text { mean of replicate analyses. }
\end{array}
$$

Accuracy will be determined as the percent recovery as follows:

$$
\% R=\frac{C_{N}}{C_{T}} \times 100
$$

where

$$
\% R=\text { percent recovery }
$$


Revision: 0

Date: June 14, 1991

Table 4. Scheduled $Q C$ and Calibration

\begin{tabular}{|c|c|c|c|}
\hline $\begin{array}{l}\text { Section } \\
\text { Number }\end{array}$ & Procedure & QC Procedure & Acceptance Criteria \\
\hline 7.2 & 3-pt ICAL & $\begin{array}{l}\text { Initially and as } \\
\text { needed }\end{array}$ & $\%$ RSD all compounds $<30 \%$ \\
\hline 7.2 & Continuing & Every 12 hours & $\begin{array}{l}\% D \text { for all compounds within } \\
30 \% \text { of ICAL }\end{array}$ \\
\hline 8.4 & $\begin{array}{l}\text { Laboratory } \\
\text { control } \\
\text { sample }\end{array}$ & $\begin{array}{l}\text { Every ICAL and one } \\
\text { per analytical batch } \\
\text { or } 5 \% \text { of all field } \\
\text { samples }\end{array}$ & Table 3 \\
\hline 8.5 & $\begin{array}{l}\text { Laboratory } \\
\text { duplicate }\end{array}$ & $\begin{array}{l}5 \% \text { of all field } \\
\text { samples }\end{array}$ & Table 3 \\
\hline 8.3 & $\begin{array}{l}\text { Laboratory } \\
\text { blank }\end{array}$ & $\begin{array}{l}\text { One per analytical } \\
\text { batch }\end{array}$ & $\begin{array}{l}\text { Target analytes }<50 \% \text { of the } \\
\text { PRQLs (Table 1) }\end{array}$ \\
\hline 8.2 & Proficiency & Every six months & Table 3 \\
\hline
\end{tabular}




$$
\begin{aligned}
& C_{M}=\text { measured concentration } \\
& C_{T}=\text { true concentration. }
\end{aligned}
$$

Method detection limit will be determined as follows:

$$
\left.M D L=\tau_{(n-1,1-1}=0.00\right) \times S
$$

where

$$
M D L=\text { method detection limit }
$$

$S \quad=$ standard deviation

$\left.t_{(n-1,1-a}=0.99\right)=t$-distribution value appropriate to a $99 \%$ confidence level and a standard deviation estimate with $(n-1)$ degrees of freedom.

Demonstration of acceptable method and laboratory performance must be repeated at a minimum of every six months. The results of method performance testing must be documented.

8.3 The laboratory must continually analyze blanks (Table 4). Using the sample preparation manifold, blanks will be generated in the laboratory by filling SUMMA canisters with humid zero air or nitrogen. These canisters will then be analyzed using the procedures established in Section 7.0. Laboratory blank results are acceptable if the amounts of individual analytes listed in Table 1 are less than $50 \%$ of the PROLs. Laboratory blanks must be analyzed with a frequency of one per analytical batch.

8.4 The laboratory must analyze laboratory control samples (LCS) on a continuing basis. Commercially purchased gas standards should be used to prepare LCSs. LCSs will be prepared in the laboratory by attaching the gas standard to the laboratory sample preparation manifold and filling evacuated canisters. For guidance on preparing LCSs, see Section 5.3 of Method Six (Modified Method T0-141. The gas standard used to prepare the LCSs must be independent of those used for instrument calibration. This standard must contain all of the analytes listed in Table 1. The concentration of analytes must be in the linear calibration range of the GC. Results will be acceptable if the criteria specified in Table 3 are met. LCSs must be analyzed with every ICAL and at a frequency of $5 \%$ of all field samples or one per analytical batch, whichever is greater. The results from LCSs must be documented. 
8.5 The laboratory must analyze individual field samples in duplicate. Field canisters will be analyzed in duplicate at a frequency of $5 \%$ of all field samples or one per analytical batch, whichever is greater. Laboratory duplicate results must meet the criteria specified in Table 3. Duplicate sample results must be documented.

8.6 The laboratory will also be required to analyze blind audit samples. These audit samples are part of the WIPP Performance Demonstration Program. Details of this program are in the WIPP Experimental-Waste Characterization Program QAPP. 


\subsection{GASES}

This Guidance Manual contains the following procedures for the analysis of waste container headspace gases:

Procedure 510.1 provides a mass spectrometric procedure for the determination of hydrogen, nitrogen, nitrogen oxides, oxygen, argon, carbon monoxide, carbon dioxide, methane, ethane, and propane in waste container headspace samples that have been collected using SUMMA ${ }^{\oplus}$ passivated canisters.

Procedure 520.1 provides a gas chromatographic procedure for the determination of hydrogen, nitrogen, oxygen, argon, carbon monoxide, carbon dioxide, methane, ethane, and propane in waste container headspace samples that have been collected using SUMMA passivated canisters.

Procedure 530.1 provides a spectrophotometric procedure for the determination of the combined nitrogen dioxide and nitric oxide concentration in waste container headspace samples that have been collected using SUMMA ${ }^{\circ}$ passivated canisters. An aliquot of the headspace sample is treated with chromic acid to oxidize all nitric oxide to nitrogen dioxide. An azo-dye forming reagent is added to the aliquot and reacts with any nitrogen dioxide present to produce a red-violet color. The intensity of the color, which is a function of the nitrogen dioxide concentration, is measured at $550 \mathrm{~nm}$ with a spectrophotometer.

Analysts must consult the most current revision of the QAPP for applicable quality assurance objectives, calibration procedures and frequencies, and data reduction, validation, and reporting requirements. 


\section{DETERMINATION OF HEADSPACE GASES BY MASS SPECTROMETRY}

\subsection{SCOPE AND APPLICATION}

1.1 This procedure covers the quantitative analysis of gases containing various combinations of the following components: hydrogen, nitrogen, nitrogen oxides (combined), oxygen, argon, carbon monoxide, carbon dioxide, methane, ethane, and propane. The quality assurance objectives for these gases are outlined in Table 1. Following the guidelines set forth in the WIPP Experimental-Waste Characterization Program QAPP and in this section will assure compliance for gas determination.

1.2 This procedure may involve hazardous materials, operations and equipment, and does not purport to address all of the safety problems associated with its use. It is the responsibility of whoever uses this procedure to consult and establish appropriate safety and health practices and determine the applicability of regulatory limitations prior to use. For specific precautionary statements, see ASTM Standard Method D-2650.

1.3 This procedure is based on mass spectrometric procedures and is restricted to use by, or under the supervision of, analysts experienced in the use of mass spectrometry and skilled in the interpretation of mass spectra.

\subsection{SUMMARY OF PROCEDURE}

2.1 The molecular species which make up a gaseous mixture are ionized and may be dissociated by electron bombardment. The positive ions of different masses thus formed are accelerated in an electrostatic field and separated in a magnetic field. The abundance of each mass present is determined from the signal at the corresponding mass-to-charge ratio. This allows the identification and quantitation of the individual constituents of the gas mixture.

\subsection{Definitions}

2.2.1 Mass Number or m/e Value of an lon: the quotient of the mass of that ion Igiven in atomic mass units) and its positive charge (number of electrons lost during ionization). 
Table 1. Gas Analysis Quality Assurance Objectives

\begin{tabular}{|c|c|c|c|c|c|}
\hline Analyte Gas & CAS Number & $\begin{array}{c}\text { Precision } \\
\text { (\%RSD or RPD) }\end{array}$ & $\begin{array}{c}\text { Accuracy } \\
(\% R)\end{array}$ & $\begin{array}{l}\text { PRDL } \\
\text { (vol\%) }\end{array}$ & $\begin{array}{c}\text { Completeness } \\
(\%)\end{array}$ \\
\hline Argon & $7440-37-1$ & $\pm 10 \%$ & $90-110 \%$ & 0.1 & $90 \%$ \\
\hline Carbon Monoxide & $630-08-0$ & $\pm 10 \%$ & $90-110 \%$ & 0.01 & $90 \%$ \\
\hline Carbon Dioxide & $124-38-9$ & $\pm 10 \%$ & $90-110 \%$ & 0.1 & $90 \%$ \\
\hline Hydrogen & $1333-74-0$ & $\pm 10 \%$ & $90-110 \%$ & 0.1 & $90 \%$ \\
\hline Nitrogen & $7727-37-9$ & $\pm 10 \%$ & $90-110 \%$ & 1.0 & $90 \%$ \\
\hline Oxygen & $7782-44-4$ & $\pm 10 \%$ & $90.110 \%$ & 0.1 & $90 \%$ \\
\hline Methane & $74-82-8$ & $\pm 10 \%$ & $90-110 \%$ & 0.1 & $90 \%$ \\
\hline Ethane & $74-84-0$ & $\pm 10 \%$ & $90-110 \%$ & 0.1 & $90 \%$ \\
\hline Propane & $74-98-6$ & $\pm 10 \%$ & $90-110 \%$ & 0.1 & $90 \%$ \\
\hline $\begin{array}{l}\text { Nitrogen Oxides } \\
\text { (combined) }\end{array}$ & & $\pm 20 \%$ & $50-150 \%$ & 0.01 & $90 \%$ \\
\hline a. Nitric Oxide & $10102-43-9$ & & & & \\
\hline b. Nitrogen Oxide & $10102-44-0$ & & & & \\
\hline
\end{tabular}


2.2.2 Relative Intensity: the ratio of a peak at any m/e relative to its base peak lusually, its parent peak).

2.2.3 Sensitivity: the height of the base peak in the spectrum of the pure compound divided by the pressure prevailing in the inlet system of the mass spectrometer immediately before opening the expansion bottle to leak.

2.2.4 Partial Pressure: the pressure of any component in the inlet system before opening the expansion bottle to leak.

\subsection{INTERFERENCES}

3.1 A gas having its principal peak at the same mass as the principal peak of another gas (CO, $\mathrm{N}_{2}$ ) may be difficult to quantitate at trace levels when the other gas is abundant.

3.2 Oxygen and hydrocarbons may interfere with the measurement of $\mathrm{CO}$ at the ppm level from the reactions of these components at the filament to form $\mathrm{CO}$ and $\mathrm{CO}_{2}$.

3.3 Gases which are strongly adsorbed on MS surfaces (e.g., $\mathrm{H}_{2} \mathrm{O}, \mathrm{NO}_{2}, \mathrm{NH}_{3}$ ) may be undetected at low levels ( $<200 \mathrm{ppm})$.

3.4 Gases which appear to desensitize the filament (e.g., $\mathrm{NO}_{2}$, SiF 4 ) may introduce uncertainties in the analysis.

3.5 Trace amounts of a gas may be undetected in the presence of larger amounts of a gas having an ion fragment which coincides with the principal peak of the trace gas (e.g., $\mathrm{CO}$ in $\mathrm{CO}_{2} ; \mathrm{C}_{2} \mathrm{H}_{4}$ in $\mathrm{C}_{2} \mathrm{H}_{6}$ ).

\subsection{APPARATUS AND MATERIALS}

4.1 Resolution: The mass spectrometer must be capable of resolving the triplet, $\mathrm{CO}^{+} / \mathrm{N}_{2}{ }^{+} / \mathrm{C}_{2} \mathrm{H}_{4}{ }^{+}$, at mass 28; the doublet, $\mathrm{NO}^{+} / \mathrm{C}_{2} \mathrm{H}_{6}{ }^{+}$, at mass 30 ; and the triplet, $\mathrm{N}_{2} \mathrm{O}^{+} / \mathrm{CO}_{2}+/ \mathrm{C}_{3} \mathrm{H}_{8}{ }^{+}$, at mass 44. Additional performance tests described herein and in ASTM E137-82 must be considered.

The mass spectrometer should have an abundance sensitivity (tail contribution at $\mathrm{m} / \mathrm{e} 29$ from a peak at m/e 28) of $1 \mathrm{ppm}$, to allow measurement of low leveis of $\mathrm{CO}$ in $\mathrm{N}_{2}$ at $\mathrm{m} / \mathrm{e} 28$. When 
abundance sensitivity is not sufficient, measuring $\mathrm{CO}$ at $\mathrm{m} / \mathrm{e} 12$ may give better results. If the parent peak is not used to determine a given analyte, details of using the alternate masses should be outlined in the SOP.

4.2 Data System: A data system must be interfaced to the MS and must allow acquisition of data at all masses of interest. At a minimum, it should be capable of reprocessing data and external standard calculations and archiving for future processing.

4.3 Sample Inlet: The sample inlet system must be configured to permit attaching SUMMA canisters directly to the MS inlet. A series of expansion reservoirs should allow the introduction of the samples at a predetermined pressure (as recommended by the manufacturer).

4.4 It is the responsibility of the analyst to verify that the instrument configuration and operating conditions satisfy the analytical requirements, and to maintain QC data confirming instrument performance and analytical results.

\subsection{REAGENTS}

5.1 Reference Standards: The mass spectrometer must be calibrated with each of the target analyte gases listed in Table 1. The calibrating compounds should be of high purity (>99.9\%). Pure gases and certified mixtures may be used. Contribution of ion fragments for all appropriate masses must be measured. In general, the mass spectrometer is capable of detecting impurities in calibrants and the contribution of such impurities to the calibration spectrum must be accounted for. This is done by measuring the concentration of the impurity and correcting measured signals for contributions due to ions in its spectrum.

\subsection{SAMPLE COLLECTION, PRESERVATION, AND HANDLING}

6.1 Samples will be collected using the procedures described in the WIPP Experimental-Waste Characterization Program QAPP.

6.2 There are no method-specific requirements for sample preservation or holding times prior to analysis. To facilitate timely reporting of the analysis data, sample holding times should not exceed 28 days. 
6.3 All sample handling and chain-of-custody procedures described in the QAPP for the WIPP Experimental-Waste Characterization Program must be followed.

\subsection{PROCEDURE}

\subsection{Recommended Operating Conditions}

7.1.1 Obtain the mass spectrum of the sample under the same conditions as the calibration spectra. Instrument settings are established to provide optimum performance for the gas mixture to be analyzed. Specific instrument settings must be listed in an SOP. Suggested instrument parameters are as follows:

$\begin{array}{ll}\text { Inlet system temperature: } & 100^{\circ} \mathrm{C} \\ \text { lon source temperature: } & 200^{\circ} \mathrm{C} \\ \text { Trap current: } & 50 \mu \text { amperes } \\ \text { Electron energy: } & 80 \mathrm{~V} \\ \text { Deflection plate voltages: } & \\ \text { YD: } & 460 \\ \text { HP: } & 713 \\ \text { ZD: } & 855 \\ \text { Source slit: } & 1.28 \text { (micrometer settings) } \\ \text { Ion repeller voltage: } & -5.0 \mathrm{~V} \\ \text { lon collectors: } & \\ \text { Axial collection slit: } & 2.30 \text { (micrometer setting 1200 RP) } \\ \text { Electron multiplier voltage: } & 2.2 \mathrm{kV} \\ \text { Daily electrode supply } & \\ \text { (Bradenburg) voltage: } & 8 \mathrm{kV}\end{array}$

The above instrument conditions are for a VG Model 30-01. These values may vary or be set automatically on different instruments.

The general procedure may involve a continuous or peak-hopping mass scan to determine if there are unexpected components in the gas mixture. This is followed by a slower scan or high precision peak-hopping mode, which allows precise measurement of the intensities of specific peaks for quantification of components in the mixture.

It shall be the responsibility of the analyst to verify that the instrument configuration and operating conditions (scanning rate, resolution, ion voltages, detector voltages, etc.) satisfy the analytical requirements, and to maintain QC data confirming instrument performance and analytical results. 


\subsection{Calibration and Standardization}

\subsubsection{Apparatus: Determine whether operating conditions remain normal by making} certain tests periodically, following instructions furnished by the manufacturer of the apparatus. Include in these tests rate of leak, magnet current, and ion-beam control settings. These tests should be performed during initial instrument setup and as specified by the manufacturer. For example, these tests may need to be performed after changing a leak valve or filament, or performing other maintenance functions.

7.2.2 Reference Standards: To calibrate the mass spectrometer, a known quantity of the calibration gas is introduced into the mass spectrometer. The field position and sensitivity are determined for each gas to be measured. Calibration for each gas which may be found in the sample should be performed.

\subsubsection{Calibration Data}

7.2.3.1 Initial Calibration: The mass spectrometer must be calibrated for mass position and sensitivity. Mass position calibration establishes a general relationship between mass and magnetic (or electric) fields over the useful mass range. Mass calibration must be done with at least four calibration gases designed to cover the useful mass range. Typical calibration masses include mass 2.016 (hydrogen), mass 4.003 (helium), mass 4.028 (deuterium), mass 14 (nitrogen), mass 28 (nitrogen), mass 40 (argon), mass 84 (krypton), and mass 129 (xenon).

Sensitivity calibration must be done for each component in the gas mixture. Pure gases or certified mixtures are used to determine the sensitivity, usually in units of $\mathrm{mV}$ per unit pressure. The reference standards should be introduced at approximately the same inlet pressure as the sample ( $\pm 10 \%$ RPD).

Mass and sensitivity calibrations are done during the initial instrument setup for all constituents to be measured. Additional calibrations should be performed as specified by the manufacturer or when standard recoveries do not meet the acceptance criteria of 90 to $100 \%$ recovery.

7.2.3.2 Continuing Calibration: Continuing calibration checks may be done with the laboratory control sample (LCS). If a gas other than the LCS is used for continuing calibration, then the LCS must be run as a sample during the analytical sequence. The continuing calibration gas must be from a separate source than that used for the initial instrument calibration. The calibration check must be run at the beginning and end of each analytical batch. The concentration of each of the analytes in the mixture is measured. If the relative percent difference between this concentration and 
the certified concentration is less than $10 \%\left(50 \%\right.$ for $\left.\mathrm{NO}_{x}\right)$, then the mass spectrometer will be considered calibrated. Calibration is also verified by showing that the sum of the measured partial pressures of the components equals the total pressure of the introduced sample (within $3 \%$ ).

\subsection{Calculations}

7.3.1 The partial pressure of each component may be calculated automatically by the instrument or manually as follows:

$$
P_{i}=\frac{P k H t}{\text { Sens }}
$$

where

$$
\begin{aligned}
& P_{i}=\text { the partial pressure of the constituent } i \text { (m Torr) } \\
& \mathrm{Pk} \mathrm{Ht}_{\mathrm{t}}=\text { the peak height in } \mathrm{mV} \\
& \text { Sens }=\text { the sensitivity of the constituent in } \mathrm{mV} \text { per unit pressure (m Torr). }
\end{aligned}
$$

The sum of the partial pressures of measured components should agree within $3 \%$ of the pressure measured in the expansion reservoir of the mass spectrometer. If water vapor is present in the sample, poorer agreement may be expected by the analyst when the data is reviewed. Poor agreement between calculated and measured pressure may indicate that a major mixture component was overlooked or that the instrument needs recalibration.

Volume percent is calculated as follows:

$$
\text { Volume } \%=\frac{P_{1}}{\Sigma P_{i}} \times 100
$$

where

$\Sigma P_{1}=$ the sum of partial pressures of all the gas constituents.

\subsection{Data Report}

7.4.1 Sample data must be reported according to the requirements specified by DOENPO. The quantitative values shall be reported in units of volume percent (vol. \%) down to the MDL. Values for analytes analyzed for but not detected shall be reported as the MDL with the required "Un flag (i.e., if the MDL is 0.001 vol. \%, report $0.001 \mathrm{U}$ ). Analytical results, including MDL values used in reporting, must be corrected for dilution. 
The numerical value assigned to the MDL for a given analyte shall include two significant figures when the MDL is greater than or equal to $10 \%$ of the corresponding PRDL. If the MDL is less than $10 \%$ of the PRDL, its numerical value shall be reported with one significant figure. (Note: Under no circumstances is a value reported as all zeros acceptable.) Analytical results above the MDL shall be reported to a sufficient number of decimal places to include the least significant figure of the MDL, but to no more than three significant figures.

To illustrate implementation of these reporting requirements, consider the example of an analyte having a PRDL of 0.1 vol. \%. If the MDL for this analyte is 0.024 vol. \%, then a result of 0.09265 would be reported as 0.093 . If the MDL is 0.0008 vol. $\%$, then 0.09265 would be reported as 0.0926 .

\subsection{QUALITY CONTROL}

8.1 This section outlines the minimum OC operations necessary to satisfy the analytical requirements of the WIPP Experimental-Waste Characterization Program QAPP. The laboratory QC frequency and acceptance criteria are summarized in Table 2 . The following $O C$ operations must be performed:

8.1.1 Instrument Calibration: Mass and sensitivity calibrations are done during intital instrument setup for all constituents to be measured. Additional calibrations should be performed as specified by the manufacturer or when standard recoveries or closures do not meet the acceptance criteria (90 to $100 \%$ recovery and $3 \%$, respectively).

8.1.2 Calibration Verification: Calibration verification may be done using a certified mixture of all the analytes in the TAG list (Table 1) or using the LCS. The verification must be done at the beginning and the end of each analytical batch. If the LCS is not used as the calibration verification standard then it must be run at least once during the analytical batch. The calibration verification will be used to verify field positions for the masses of interest. Closure values on this check must not fail by more than $3 \%$ and the values of the individual analytes must be within $10 \%$ of their accepted values $\left(50 \%\right.$ for $\left.\mathrm{NO}_{x}\right)$. If the calibration check does not meet these criteria, then remedial action must be taken and the samples must be reanalyzed to the last good check standard.

\subsubsection{Blank Analysis}

8.1.3.1 Laboratory Blanks (LBs): The LBs will consist of ultra-high purity nitrogen or helium $(99.999 \%$ pure). The same analytical method used to prepare the field 
Table 2. Scheduled $\mathrm{OC}$ and Calibration

\begin{tabular}{|c|c|c|c|}
\hline $\begin{array}{l}\text { Section } \\
\text { Number }\end{array}$ & Procedure & $\begin{array}{l}\text { Frequency of } \\
\text { OC Procedure }\end{array}$ & Acceptance Criteria \\
\hline 8.1 .1 & $\begin{array}{l}\text { Initial } \\
\text { calibration }\end{array}$ & $\begin{array}{l}\text { Initially and } \\
\text { as needed }\end{array}$ & $\begin{array}{l}\text { \%RSD of all compounds }<35 \% \text {; } \\
\text { RRTs within } 0.06\end{array}$ \\
\hline 8.1 .2 & $\begin{array}{l}\text { Continuing } \\
\text { calibration }\end{array}$ & $\begin{array}{l}\text { At the beginning } \\
\text { and end of each } \\
\text { analytical sequence }\end{array}$ & $\begin{array}{l}\% D \text { for all compounds } \\
\text { within } 10 \% \text { of initial } \\
\text { calibration }(50 \% \text { for NOx) }\end{array}$ \\
\hline 8.1 .5 & $\begin{array}{l}\text { Laboratory } \\
\text { control } \\
\text { sample }\end{array}$ & $\begin{array}{l}\text { One per analytical } \\
\text { batch }\end{array}$ & $\begin{array}{l} \pm 10 \% \text { recovery of certified } \\
\text { known value }\end{array}$ \\
\hline 8.1 .4 & $\begin{array}{l}\text { Laboratory } \\
\text { duplicate }\end{array}$ & $\begin{array}{l}\text { One per analytical } \\
\text { batch }\end{array}$ & $\begin{array}{l}\% D \text { for all compounds } \\
\text { - within } 10 \% \text { of sample } \\
\text { results }(20 \% \text { for NOx) }\end{array}$ \\
\hline 8.1 .3 & $\begin{array}{l}\text { Laboratory } \\
\text { blank }\end{array}$ & $\begin{array}{l}\text { One per analytical } \\
\text { batch }\end{array}$ & $\begin{array}{l}\text { Target analytes less than } \\
\text { the PRDLs (Table } 1 \text { ) }\end{array}$ \\
\hline 8.2 & $\begin{array}{l}\text { Proficiency } \\
\text { testing }\end{array}$ & $\begin{array}{l}\text { Each laboratory, } \\
\text { every six months }\end{array}$ & \\
\hline
\end{tabular}


samples for analysis will be used to prepare and analyze the LBs. LB results will be acceptable if the concentrations of the TAGs (except nitrogen) are less than the PRDLs. Remedial action must be taken for blanks which exceed the PRDLs. LBs will be analyzed daily during analytical operations.

\subsubsection{Duplicate Analysis}

8.1.4.1 Laboratory Duplicate: The laboratory shall analyze field samples in duplicate. Single canisters shall be analyzed in duplicate at a frequency of one per analytical batch. The site Project Manager may request additional duplicate sample analysis.

Single canisters analyzed in duplicate shall be used to assess laboratory precision. Laboratory duplicate results shall be considered acceptable for gases if the RPD is less than $\pm 10 \%$ for concentrations greater than 10 times the PRDL, and the absolute difference is less than $\pm P R D L$ for concentrations less than 10 times the PRDL. Laboratory duplicates for $\mathrm{NO}_{x}$ shall be acceptable if the RPD is less than $\pm 20 \%$ for concentrations greater than 5 times the PRDL, and the absolute difference is less than \pm PRDL for concentrations less than 5 times the PRDL. - Duplicates which do not meet these criteria should be flagged. It will be the responsibility of the laboratory QA Officer to monitor and document duplicate results.

\subsubsection{Laboratory Control Sample (LCS): The LCS is used to monitor the laboratory's day-} to-day performance of routine analytical methods. An LCS consists of a certified standard gas mixture and must be from sources independent of those used for instrument calibration. The LCS is analyzed with field gas samples to provide evidence that the laboratory is performing the method within accepted OC guidelines.

The LCS must be analyzed at least once per analytical batch. The LCS analysis is designed to ensure that laboratory performance $\mathrm{OC}$ is available with each batch of samples processed. If the LCS recovery is greater than $\pm 10 \%$ of the certified known value, then the system must be recalibrated and all of the samples in that batch must be reanalyzed.

8.2 All laboratories using these procedures must demonstrate acceptable performance prior to the analysis of any samples. Demonstration of acceptable performance will be achieved by analyzing commercially available (Scott Specialty Gases or equivalent) gas standards. These standards will contain all of the analytes listed in Table 1 at concentrations appropriate (2.5 times the PRDLs) to determine the parameters specified in Table 1. The analysis of four replicate samples must meet the criteria specified for precision, accuracy and method detection limits in Table 1. Demonstration of acceptable method and analyst performance must be repeated at a minimum of every six months. It will be the responsibility of the laboratory QA Officer at each participating laboratory to monitor and 
document method and analyst performance. The laboratory $Q A$ Officer will have responsibility to implement corrective actions when acceptable method performance is not met.

8.3 The laboratory will also be required to analyze blind audit samples. These audit samples are part of the WIPP Performance Demonstration Program. Details of this program are in the Performance Demonstration Program Plan.

\subsection{REFERENCES}

1. "D-2650 Standard Test Method for Chemical Composition of Gases by Mass Spectroscopy," Annual Book of ASTM Standards, Vol. 5.02.

2. "E-137 Practice for Evaluation of Mass Spectrometers for Quantitative Analysis from a Batch Inlet," Annual Book of ASTM Standards, Vol. 14.01. 
PROCEDURE 520.1

\section{DETERMINATION OF HEADSPACE GASES BY GAS CHROMATOGRAPHY}

\subsection{SCOPE AND APPLICATIONS}

1.1 This procedure covers the determination of the chemical composition of headspace gases and similar gaseous mixtures containing the following components: hydrogen; oxygen; nitrogen; argon; carbon monoxide; carbon dioxide; methane; ethane; and propane. The quality assurance objectives for these gases are outlined in Table 1. Following the guidelines set forth in the WIPP ExperimentalWaste Characterization Program QAPP and in this section will assure compliance for gas determinations.

1.2 This procedure may involve the use of hazardous materials, operations, and equipment and does not purport to address all of the safety problems associated with its use. It is the responsibility of whoever uses this procedure to consult and establish appropriate safety and health practices prior to use.

1.3 This procedure is based on gas chromatographic procedures and is restricted to use by, or under the supervision of, analysts experienced in the use of GC instrumentation and skilled in the interpretation of gas chromatograms.

\subsection{SUMMARY OF PROCEDURE}

2.1 Components in a sample of headspace gas are physically separated by gas chromatography and compared to corresponding components of a reference standard separated under identical operating conditions, using a reference standard mixture of known composition. The composition of the headspace gas is calculated by comparison of either the peak height or area response of each component with the corresponding value of that component in the reference standard.

\subsection{INTERFERENCES}

3.1 Helium can interfere with the hydrogen determination. 
Table 1. Quality Assurance Objectives of Analytes for Gas Analysis

\begin{tabular}{llcccc}
\hline Compound & CAS Number & $\begin{array}{c}\text { Precision } \\
\text { (\%RSD or RPD) }\end{array}$ & $\begin{array}{c}\text { Accuracy } \\
(\% R)\end{array}$ & $\begin{array}{c}\text { PRDL } \\
(\text { vol } \%)\end{array}$ & $\begin{array}{c}\text { Completeness } \\
(\%)\end{array}$ \\
\hline Argon & $7440-37-1$ & $\pm 10 \%$ & $90-110 \%$ & 0.1 & $90 \%$ \\
Carbon Monoxide & $630-08-0$ & $\pm 10 \%$ & $90-110 \%$ & 0.01 & $90 \%$ \\
Carbon Dioxide & $124-38-9$ & $\pm 10 \%$ & $90-110 \%$ & 0.1 & $90 \%$ \\
Hydrogen & $1333-74-0$ & $\pm 10 \%$ & $90-110 \%$ & 0.1 & $90 \%$ \\
Nitrogen & $7727-37-9$ & $\pm 10 \%$ & $90-110 \%$ & 1.0 & $90 \%$ \\
Oxygen & $7782-44-4$ & $\pm 10 \%$ & $90-110 \%$ & 0.1 & $90 \%$ \\
Methane & $74-82-8$ & $\pm 10 \%$ & $90-110 \%$ & 0.1 & $90 \%$ \\
Ethane & $74-84-0$ & $\pm 10 \%$ & $90-110 \%$ & 0.1 & $90 \%$ \\
Propane & $74-98-6$ & $\pm 10 \%$ & $90-110 \%$ & 0.1 & $90 \%$ \\
\hline \hline
\end{tabular}

$\%$ RSD $=$ Percent relative standard deviation

$\% R P D=$ Percent recovery

$\% R=$ Percent recovery

PRDL = Program Required Detection Limit (Volume Percent) 


\subsection{APPARATUS AND MATERIALS}

4.1 Chromatograph: Chromatographic instrument and required accessories including detectors, column supplies, a data system, a pressure measuring device, and a fixed volume sample loop. The data system must be interfaced to the GC and must allow continuous acquisition of data throughout the duration of the chromatographic program. At a minimum, it must be capable of reprocessing data and external standard calculations and archiving for future processing.

4.2 Detector: The detector shall be a thermal conductivity type, or its equivalent in stability and sensitivity for the target analytes. The thermal conductivity detector must be sufficiently sensitive to produce a signal of at least $0.5 \mathrm{mV}$ for 1 mole percent methane in a $0.5 \mathrm{~mL}$ sample.

4.3 Detector Temperature Control: The detector temperature shall be maintained at a temperature constant to $0.3^{\circ} \mathrm{C}$ (as specified by the manufacturer) during the course of the sample run and the corresponding reference run. The detector temperature shall be equal to, or greater than, the maximum column temperature.

\subsection{Sample Inlet System: Constant Volume Gas Sampling Loop}

4.4.1 The system must be capable of attaching SUMMA canisters directly to the sample inlet. The sample inlet system must be constructed of materials that are inert and nonadsorptive with respect to the components in the sample. The preferred material of construction is stainless steel. Copper and copper-bearing alloys are not acceptable. Loss of sample due to excessive transfer line volume should be minimized. The inlet system should allow pressure measurements to $0.1 \mathrm{kPa}$ and temperature measurements to $1^{\circ} \mathrm{C}$. The inlet system should be operated at ambient temperature.

4.4.2 Provision must be made to introduce into the carrier gas ahead of the analyzing column a gas-phase sample that has been entrapped in either a fixed volume loop or tubular section. The sample injection valves may be configured such that the sample is swept by carrier gas directly onto the GC column or into a split/splitless injection port. The temperature, pressure, and volume of the sample must be known. To prevent sample contamination, the sample loop and tubing to the sample loop must be capable of being evacuated or purged for the purpose of cleaning or lowering background. 


\subsection{Columns}

4.5.1 The columns shall be constructed of materials that are inert and nonadsorptive with respect to the components in the sample. The preferred material of construction is stainless steel. Copper and copper-bearing alloys are not acceptable.

4.5.2 Either an adsorption-type column (e.g., molecular sieve) or a partition-type column (e.g., Porapak Q), or both, may be used to conduct the analysis. Cooled capillary columns may also be used.

See ASTM Standard E-260 for general gas chromatography procedures.

4.5.2.1 Adsorption Column: This column must completely separate helium, hydrogen, argon, oxygen, nitrogen, methane, ethane, propane, and carbon monoxide. Demonstration of separation is required. Figure 1 is an example chromatogram obtained with an adsorption column.

1. Because of similarities in thermal conductivities, helium should not be used as the carrier gas for hydrogen when hydrogen is less than $1 \%$ of the sample. Neon carrier gas is suitable for both percent and parts per million quantities of hydrogen.

2. The use of a carrier gas mixture of $8.5 \%$ hydrogen and $91.5 \%$ helium will avoid the problem of reversing polarities of hydrogen responses as the concentration of hydrogen in the sample is increased.

3. The sensitivity of measurement of hydrogen can be increased by using a separate injection for hydrogen, using either argon or nitrogen for the carrier gas.

4. Another technique for isolating the hydrogen in a sample is to use a palladium transfer tube at the end of the adsorption column; this will permit only hydrogen to be transferred to a stream of argon or nitrogen carrier gas for analysis in a second thermal conductivity detector.

4.5.2.2 Partition Column: This column must separate methane, ethane, propane, and carbon dioxide. Demonstration of separation is required. Figure 2 is an example chromatogram obtained with a partition column.

4.5.3 General: Those column materials operated either isothermally or with temperature programming, or both, may be utilized if they provide satisfactory separation of all components. If 
Revision: 0

Date: June 14, 1991

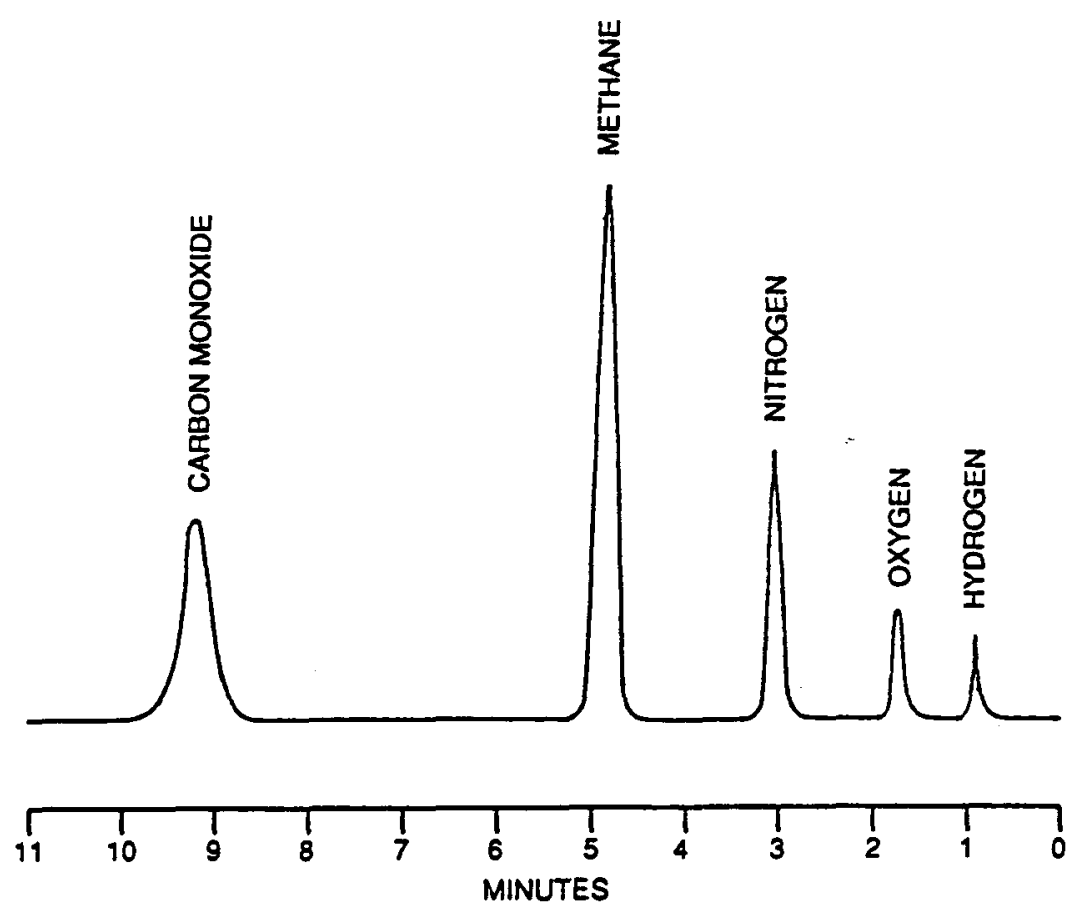

Column: $2 \mathrm{~m}$ by $6 \mathrm{~mm}$ insioe diameter Type $13 x$ molecular sieves, 14 to 30 mesh Temperature: $35^{\circ} \mathrm{C}$

Flow rate: $60 \mathrm{~mL}$ helium/min

Sampie size: $0.5 \mathrm{~mL}$

Figure 1. Chromatogram of Reformed Gas on Molecular Sieve Column 
Revision: 0

Date: June 14, 1991

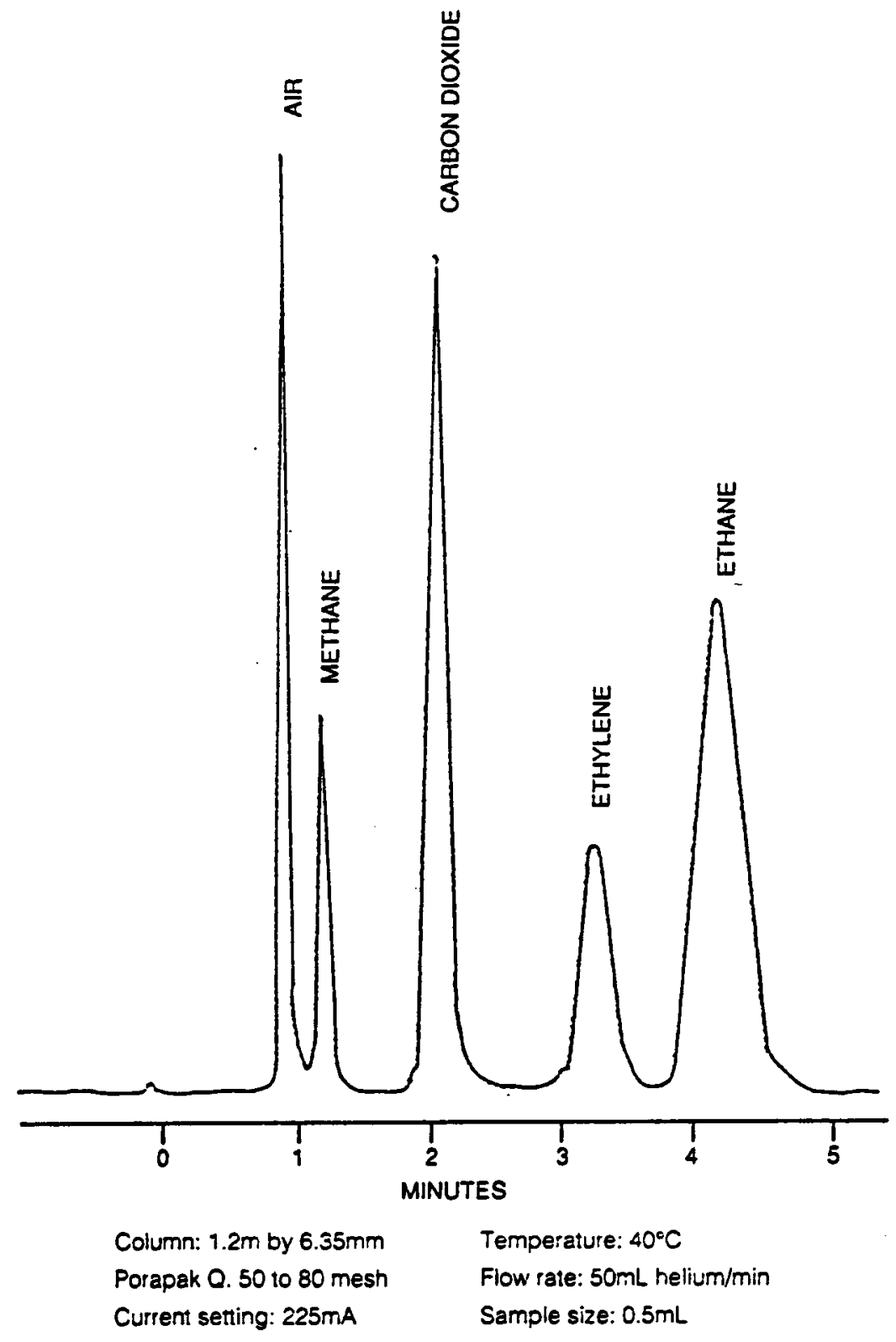

Figure 2. Chromatogram of Reformed Gas on Porapak Q Column (Reproduced from ASTM D 1946) 
complete separation is not possible with packed columns, multiple injections using different columns and/or conditions may be used. Capillary columns at reduced temperatures may also be used.

\subsection{Column Temperature Control}

4.6.1 Isothermal: When isothermal operation is utilized, the analytical columns shall be maintained at a temperature constant to $0.3^{\circ} \mathrm{C}$ (as specified by the manufacturer) during the course of the sample run and the corresponding reference run.

\subsubsection{Temperature Programming: Temperature programming may be used, as appro-} priate. The oven or subambient temperature shall not exceed the recommended temperature limits for the materials in the column.

4.7 Carrier Gas: The instrument shall be equipped with suitable facilities to provide flow of carrier gas through the analyzer and detector under isothermal conditions at a flow rate that is constant to $1 \%$ throughout the analysis of the sample and the reference standard. The purity of the carrier gas may be improved by flowing the carrier gas through selective filters prior to its entry into the chromatograph.

\subsection{REAGENTS}

5.1 Reference Standards: Certified gas mixtures of known composition are required for making a standard calibration curve. They must contain known percentages of the analytes listed in Table 1. All components in the reference standard must be homogeneous in the vapor state at the time of use and should be traceable to a nationally recognized standard.

\subsection{SAMPLE COLLECTION, PRESERVATION, AND HANDLING}

6.1 Samples will be collected using the sampling procedures described in the WIPP Experimental-Waste Characterization Program QAPP.

6.2 There are no method specific requirements for sample preservation or holding times prior to analysis. To facilitate timely reporting of the analysis data, sample holding times should not exceed 28 days. 
6.3 All sample handling and chain-of-custody procedures described in the QAPP for the WIPP Experimental-Waste Characterization Program must be followed.

\subsection{PROCEDURE}

\subsection{GC Analytical System Operating Conditions}

7.1.1 Column Preparation: Prepacked or capillary columns are generally recommended (mole sieve 5a, poraPLOT $\mathrm{Q}$ or equivalent). Variations in column material, dimensions, and mesh sizes of packing are permissible if the columns produce the desired separations as shown in Figures 1 and 2.

\subsubsection{Operating Conditions (Recommended):}

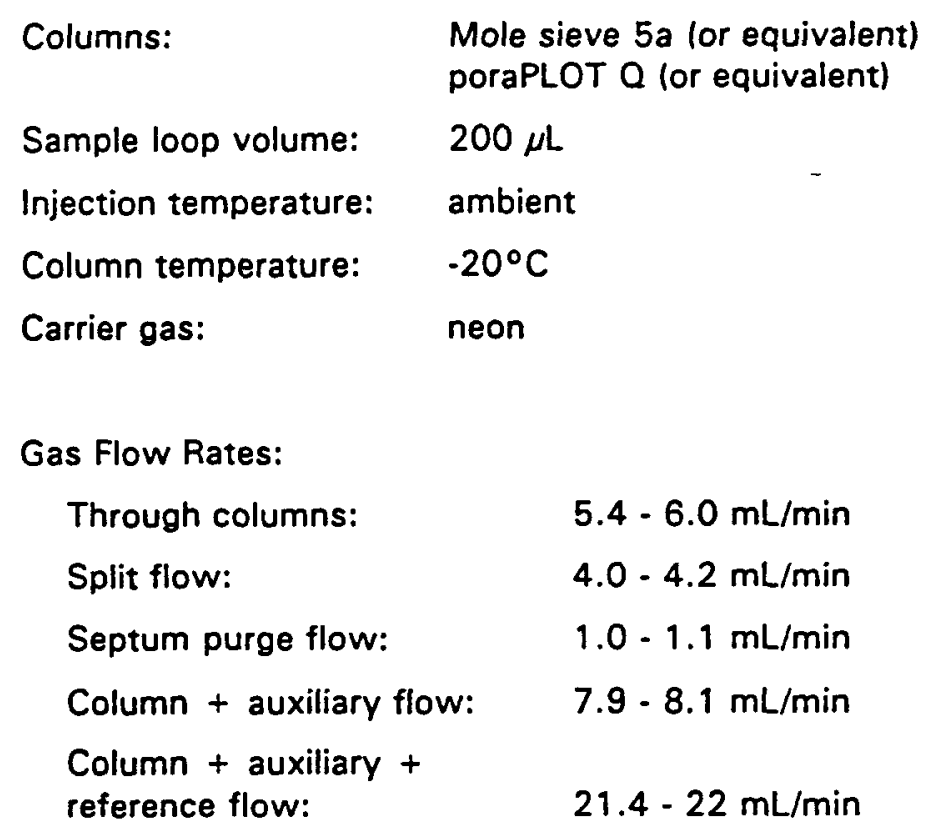

The above operating conditions are for a HP 5890 gas chromatograph. These values vary depending on the GC used.

It shall be the responsibility of the analyst to verify the instrument configuration and operating conditions (sample volume, column type, temperatures, flow rates, etc.) used to satisfy the analytical requirements and to maintain $\mathrm{QC}$ data confirming instrument performance and analytical results.

\subsection{Calibration Curves and Verification}

7.2.1 Calibration Curves: A calibration curve is constructed from a minimum of three certified gas standards. The resulting calibration curve must yield a straight line with correlation 
coefficient $(R) \geq 0.995$. The low standard should be at five times the MDL or the PRDL (Table 1), whichever is higher, and the high standard will be such that it brackets the expected sample concentrations and remains in the linear range of the instrument.

All standards and samples must be analyzed under the same operating conditions. Tabulate peak area response of the analyte against the amount injected. Calculate a response factor (RF) for each compound as follows:

$$
R F=\frac{A_{x}}{C_{x}}
$$

where

$$
\begin{aligned}
& A_{x}=\text { Total area response for the compound } \\
& C_{x}=\text { Amount (mole) of the compound being measured. }
\end{aligned}
$$

To calculate the number of moles of each gas, the following equation may be used:

$$
C_{x}=\frac{(V o l \%) V_{s} P_{s}}{R T_{s}}
$$

where:

$$
\begin{aligned}
& \text { Vol\% }=\text { Volume percent of the gas in the standard } \\
& V_{s} \quad=\text { Volume of the sample loop }(\mathrm{mL}) \\
& P_{s} \quad=\text { Pressure of the sampling loop (atm) } \\
& R \quad=82.057 \mathrm{~mL} \text { atm } /(\mathrm{K} \text { mole) } \\
& T_{s} \quad=\text { Temperature of the sampling loop (K). }
\end{aligned}
$$

Alternately, the standards may be converted to values at standard temperature and pressure. The sample inlet pressure may vary, and this variation must be compensated.

7.2.2 Calibration Verification: Calibration check standards must be analyzed at the beginning of every 12 hours of operation and prior to the analysis of any samples to verify working curves and retention time windows. The response factor (or concentration) of the continuing calibration check and retention time window (Section 7.3) will be compared to the corresponding values from the most recent valid calibration.

7.2.2.1 The verification procedure involves calculating the RF for each compound in the calibration verification standard and comparing these to the average RFs for each compound 
from the initial calibration sequence. The validity of the initial calibration RF is checked by calculating the relative percent difference for individual compounds as follows:

$$
\% D=\frac{\left(R F_{1}\right)-\left(R F_{c}\right)}{\left(R F_{1}\right)} \times 100
$$

where

$$
\begin{aligned}
& \% D=\text { Relative percent difference } \\
& R F_{1}=\text { Average RF from initial calibration } \\
& R F_{c}=\text { Response factor from the check standard. }
\end{aligned}
$$

Alternately, the value of the calibration verification may be calculated and the percent recovery of the expected value determined.

7.2.2.2 The absolute retention time for each component must fall within the retention time window (Section 7.3) established for that compound during the most recent initial calibration. If the retention time window criterion is not met, corrective action must be taken. If no source of the problem can be determined after corrective action has been taken, a new three-point initial calibration curve must be generated.

\subsection{Retention Time Window}

7.3.1 Retention time windows for each compound are determined by calculating the standard deviation of the absolute retention time for each compound over the three initial calibration standards. The retention time window shall be defined as plus or minus three times the standard deviation of the absolute retention time of each compound determined from the most recent initial calibration.

7.3.2 Retention time windows for each compound must be determined whenever a new initial calibration curve is generated or when a new GC column is installed. Retention time windows must be established for all gases in Table 1.

7.3.3 For the calibration verification standard, the midpoint of the retention time window shall be the average absolute retention time for each compound from the most recent initial calibration.

7.3.4 The midpoint of the retention time shall be the absolute retention time for each analyte from the calibration verification standard for that calibration period. 


\subsection{Data Interpretation}

7.4.1 Qualitative Analysis: The gases listed in Table 1 are identified by retention time. The retention time must be within the retention time window for the most recent calibration verification standard.

\subsubsection{Quantitative Analysis}

7.4.2.1 Once a compound has been qualitatively identified, the quantitation of that compound will be based on the area response. The column used for quantitation should be interferant-free in the retention time window corresponding to the analyte.

7.4.2.2 Calculate the concentration in volume percent of each identified analyte in the sample as follows:

$$
\text { Concentration (vol. \%) }=\frac{A_{x}(R)\left(T_{y}\right)}{(R F)\left(V_{2}\right)\left(P_{2}\right)}
$$

with the variables as described in equations (1) and (2).

If equations (1) and (2) were not used to calculate the response factors, the samples must be adjusted for sample loop pressure in the same manner as the standards.

7.5 Data Report: Sample data must be reported according to the requirements specified by DOENWPO. The quantitative values shall be reported in units of volume percent (vol. \%) down to the MDL. Values for analytes analyzed for but not detected shall be reported as the MDL with the required "U" flag (i.e., if the MDL is 0.001 vol. \%, report $0.001 \mathrm{U}$ ). Analytical results, including MDL values used in reporting, must be corrected for dilution.

The numerical value assigned to the MDL for a given analyte shall include two signficant figures when the MDL is greater than or equal to $10 \%$ of the corresponding PRDL. If the MDL is less than $10 \%$ of the PRDL, its numerical value shall be reported with one significant figure. (Note: Under no circumstances is a value reported as all zeros acceptable.) Analytical results above the MDL shall be reported to a sufficient number of decimal places to include the least significant figure of the MDL, but to no more than three significant figures.

To illustrate implementation of these reporting requirements, consider the example of an analyte having a PRDL of $0.1 \mathrm{vol} . \%$. If the MDL for this analyte is 0.024 vol. \%, then a result of 0.09265 would be reported as 0.093 . If the MDL is 0.0008 vol. \%, then 0.09265 would be reported as 0.0926 . 


\subsection{QUALITY CONTROL}

8.1 This section outlines the minimum necessary OC operations to satisfy the analytical requirements of the WIPP-QAPP. The laboratory $O C$ frequency and acceptance criteria are summarized in Table 2. The following $\mathrm{QC}$ operations must be performed:

8.1.1 Calibration Curves: A calibration curve is constructed from a minimum of three certified gas standards and a blank. The resulting calibration curve must yield a straight line with a correlation coefficient $(R) \geq 0.995$. The low standard should be at five times the MDL or the PRDL (Table 1), whichever is higher, and the high standard will be such that it brackets the expected sample concentrations and remains in the linear range of the instrument.

8.1.2 Calibration Verification: Calibration checks will be run at the beginning of each 12 hour shift. The RF (or concentration) of the continuing calibration check will be compared to the corresponding average RF (or concentration) from the most recent valid calibration. If the RPD between the average response factor and the continuing calibration check is less than $10 \%$, the GC system will be considered calibrated. If it is not within the control limits, then the problem should be identified and the instrument must be recalibrated.

\subsubsection{Blank Analysis}

8.1.3.1 Laboratory Blanks (LBs): SUMMA' canisters will be filled with ultra high purity nitrogen $(99.999 \%$ pure) in the laboratory using the sample preparation manifold. The same analytical procedure used to prepare the field samples for analysis will be used to prepare the laboratory blanks. Laboratory blank results will be acceptable if the concentrations of the TAGs (except nitrogen) are less than the PRDLs. Remedial action must be taken for blanks which exceed the PRDL. Laboratory blanks will be analyzed daily during analytical operations.

\subsubsection{Duplicate Analysis}

8.1.4.1 Laboratory Duplicate: The laboratory shall analyze field samples in duplicate. Single canisters shall be analyzed in duplicate at a frequency of one per analytical batch. Single canisters analyzed in duplicate shall be used to assess laboratory precision. Laboratory duplicate results shall be considered acceptable for gases if the RPD is less than $\pm 10 \%$ for concentrations greater than 10 times the PRDL, and the absolute difference is less than \pm PRDL for concentrations less than 10 times the PRDL. The RPD for duplicate analyses is the difference between the 
Table 2. Scheduled $\mathrm{QC}$ and Calibration

\begin{tabular}{|c|c|c|c|}
\hline $\begin{array}{l}\text { Section } \\
\text { Number }\end{array}$ & Procedure & $\begin{array}{l}\text { Frequency of } \\
\text { OC Procedure }\end{array}$ & Acceptance Criteria \\
\hline 8.1 .1 & $\begin{array}{l}\text { Initial } \\
\text { calibration }\end{array}$ & $\begin{array}{l}\text { Initially and } \\
\text { as needed }\end{array}$ & $R>0.995$ \\
\hline 8.1 .2 & $\begin{array}{l}\text { Continuing } \\
\text { calibration }\end{array}$ & 12 hours & $\begin{array}{l}\% D \text { for all compounds } \\
\text { within } 10 \% \text { of initial } \\
\text { calibration }\end{array}$ \\
\hline 8.1 .5 & $\begin{array}{l}\text { Laboratory } \\
\text { control } \\
\text { sample }\end{array}$ & $\begin{array}{l}\text { One per analytical } \\
\text { batch }\end{array}$ & $\begin{array}{l} \pm 10 \% \text { recovery of certified } \\
\text { known value }\end{array}$ \\
\hline 8.1 .4 & $\begin{array}{l}\text { Laboratory } \\
\text { duplicate }\end{array}$ & $\begin{array}{l}\text { One per analytical } \\
\text { batch }\end{array}$ & $\begin{array}{l}\% \mathrm{D} \text { for all compounds } \\
\text { within } 10 \% \text { of sample } \\
\text { results }\end{array}$ \\
\hline 8.1 .3 & $\begin{array}{l}\text { Laboratory } \\
\text { blank }\end{array}$ & $\begin{array}{l}\text { One per analytical } \\
\text { batch }\end{array}$ & $\begin{array}{l}\text { Target analytes less than } \\
\text { the PRDLs (Table 1) }\end{array}$ \\
\hline 8.2 & $\begin{array}{l}\text { Proficiency } \\
\text { testing }\end{array}$ & $\begin{array}{l}\text { Each laboratory, } \\
\text { every six months }\end{array}$ & \\
\hline
\end{tabular}


two values divided by the average times 100 . Duplicates which do not meet these criteria should be flagged. It will be the responsibility of the laboratory QA Officer to monitor and document the results.

8.1.5 Laboratory Control Sample (LCS): The LCS is used to monitor the laboratory's dayto-day performance of routine analytical methods. An LCS consists of a standard gas mixture and must be from sources independent of those used for instrument calibration. The LCS is analyzed with WIPP gas samples to provide evidence that the laboratory is performing the procedure within accepted QC guidelines.

The LCS must be analyzed at least once per analytical batch. The LCS analysis is designed to ensure that laboratory performance $\mathrm{OC}$ is available with each batch of samples processed. If the LCS recovery is greater than $\pm 10 \%$ of the certified known value, the system must be recalibrated and all of the samples in that batch must be reanalyzed.

8.2 All laboratories using these methods must demonstrate acceptable performance prior to the analysis of any samples. Demonstration of acceptable performance will be achieved by analyzing commercially available (Scott Specialty Gases or equivalent) gas standards. These standards will contain all of the analytes listed in Table 1 at concentrations appropriate (two to five times the PRDLs) to determine the parameters specified in Table 1. The analysis of four replicate samples must meet the criteria specified for precision, accuracy, and method detection limits in Table 1. Demonstration of acceptable method and analyst performance must be repeated at a minimum of every six months. It shall be the responsibility of the QA Officer at each participating laboratory to monitor and document method and analyst performance. The laboratory QA Officer shall have the responsibility to implement corrective actions when acceptable method performance is not met.

8.3 The laboratory will be required to analyze blind audit samples. These audit samples are part of the WIPP Performance Demonstration Program Plan. Details of this Program are in the Performance Demonstration Program Plan.

\subsection{REFERENCES}

1. "D-1946 Standard Method for Analysis of Reformed Gas by Gas Chromatography," Annual Book of ASTM Standards, Vol 5.05.

2. "E-260 Recommended Practice for General Gas Chromatography Procedures," Annual Book of ASTM Standards, Vol 14.01. 


\section{PROCEDURE 530.1}

\section{DETERMINATION OF THE COMBINED NITROGEN OXIDES CONTENT OF HEADSPACE GAS SAMPLES}

\subsection{SCOPE AND APPLICATION}

1.1 This procedure covers the determination of the combined nitrogen dioxide $\left(\mathrm{NO}_{2}\right)$ and nitric oxide (NO) content in headspace gas in the range from 10 to 1000 ppm. Samples must be reported in volume percent according to the quality assurance objectives shown in Table 1. Following the guidelines set forth in the WIPP Experimental-Waste Characterization Program QAPP and in this section will assure compliance for gas determination.

\subsection{SUMMARY OF PROCEDURE}

2.1 NO in a gas phase sample is quantitatively converted to $\mathrm{NO}_{2}$ by a chromic acid oxidizer. The resulting $\mathrm{NO}_{2}$, plus the $\mathrm{NO}_{2}$ already present, are absorbed in an azo-dye-forming reagent. A redviolet color is produced within 15 min, the intensity of which is measured spectrophotometrically at $550 \mathrm{~nm}$.

\subsection{INTERFERENCES}

3.1 Any significant interferences due to sulfur dioxide $\left(\mathrm{SO}_{2}\right)$ should be negated by the oxidation step. The addition of acetone to the reagent retards color-fading by forming a temporary addition product with $\mathrm{SO}_{2}$. This will protect the reagent from incidental exposure to $\mathrm{SO}_{2}$ and will permit reading the color intensity for 4 to $5 \mathrm{~h}$ (instead of $45 \mathrm{~min}$ without acetone) without appreciable losses.

3.2 A five-fold ratio of ozone to $\mathrm{NO}_{2}$ will cause a small interference, the maximal effect occurring in $3 \mathrm{~h}$. The reagent assumes a slightly orange tint.

3.3 The interferences from nitrous oxide and nitrogen pentoxide, and other gases that might be found in polluted air are considered to be negligible. 
Table 1. Quality Assurance Objectives

\begin{tabular}{|c|c|c|c|c|c|}
\hline Analyte Gas & CAS No. & $\begin{array}{c}\text { Precision } \\
\text { (\%RSD or RPD) }\end{array}$ & $\begin{array}{c}\text { Accuracy } \\
(\% R)\end{array}$ & $\begin{array}{c}\text { PRDL } \\
\text { (vol. \%) }\end{array}$ & $\begin{array}{c}\text { Completeness } \\
(\%)\end{array}$ \\
\hline $\begin{array}{l}\text { Nitrogen Oxides } \\
\text { (combined) }\end{array}$ & & $\pm 20 \%$ & $\pm 50 \%$ & $0.01 \%$ & $90 \%$ \\
\hline $\begin{array}{l}\text { Nitric Oxide } \\
\text { Nitrogen Oxide }\end{array}$ & $\begin{array}{l}10102-43-9 \\
10102-44-0\end{array}$ & & & & \\
\hline $\begin{array}{r}\% R= \\
\% R P D= \\
\text { RPD }= \\
\text { PRDL }=\end{array}$ & $\begin{array}{l}=\text { Percent recov } \\
=\text { Relative stanc } \\
=\text { Relative perce } \\
=\text { Program requ }\end{array}$ & $\begin{array}{l}\text { ery } \\
\text { ard deviation } \\
\text { nt difference } \\
\text { red detection limit }\end{array}$ & & & \\
\hline
\end{tabular}




\subsection{APPARATUS}

4.1 Absorber: An all-glass bubbler with a $60 \mu \mathrm{m}$ maximum pore diameter frit, commonly labeled "coarse," similar to that illustrated in Figure 1.

4.1.1 The porosity of the fritted bubbler, as well as the sampling flow rate, affect absorption efficiency. The porosity may be determined in accordance with ASTM Standard E128. An efficiency of over $95 \%$ may be expected with a flow rate of 0.4 liters $/ \mathrm{min}$ or less and a maximum pore diameter of $60 \mu \mathrm{m}$. Frits having a maximum pore diameter less that $60 \mu \mathrm{m}$ will have a higher efficiency but will require an inconvenient pressure drop for sampling.

4.1.2 If the frit is clogged, visibly discolored, or calibration standards fail, carefully clean with concentrated chromic-sulfuric acid mixture, rinse well with water, and redetermined the maximum pore diameter, as per ASTM E128.

4.1.3 Rinse the bubbler thoroughly with water and allow to dry before using.

4.2 Oxidizing Tube: $8^{\prime \prime}$ glass tube $(8 \mathrm{~mm}$ diameter $)$ containing $10 \mathrm{~cm}$ of an oxidizing compound on a support medium (MS4A molecular sieve or equivalent [see 5.2.3]) and plugged at both ends with glass wool.

4.3 Vacuum Pump: Must be capable of easily attaining $<10$ microns pressure in the sample cylinder (Figure 2).

4.4 Sample Cylinder: Stainless steel cylinder ( 40 to $75 \mathrm{ml}$ volume) with Nupro SS-2H2 or its equivalent valves at both ends. The sample cylinder is used for isolating a known quantity of sample or standard from a SUMMA canister (Figure 2) to be bubbled through an absorbing solution.

4.5 Air-metering Device: A calibrated glass variable-area flowmeter, or dry gas meter coupled with a flow indicator capable of measuring a flow of 100 to $200 \mathrm{~mL} / \mathrm{min}$ is suitable (GasMet or equivalent).

4.6 Vacuum/Pressure Gauge: Omega Model DP-87 or equivalent.

4.7 Thermometer: For measuring room temperature to $\pm 1^{\circ} \mathrm{C}$. 
Exhaust Line

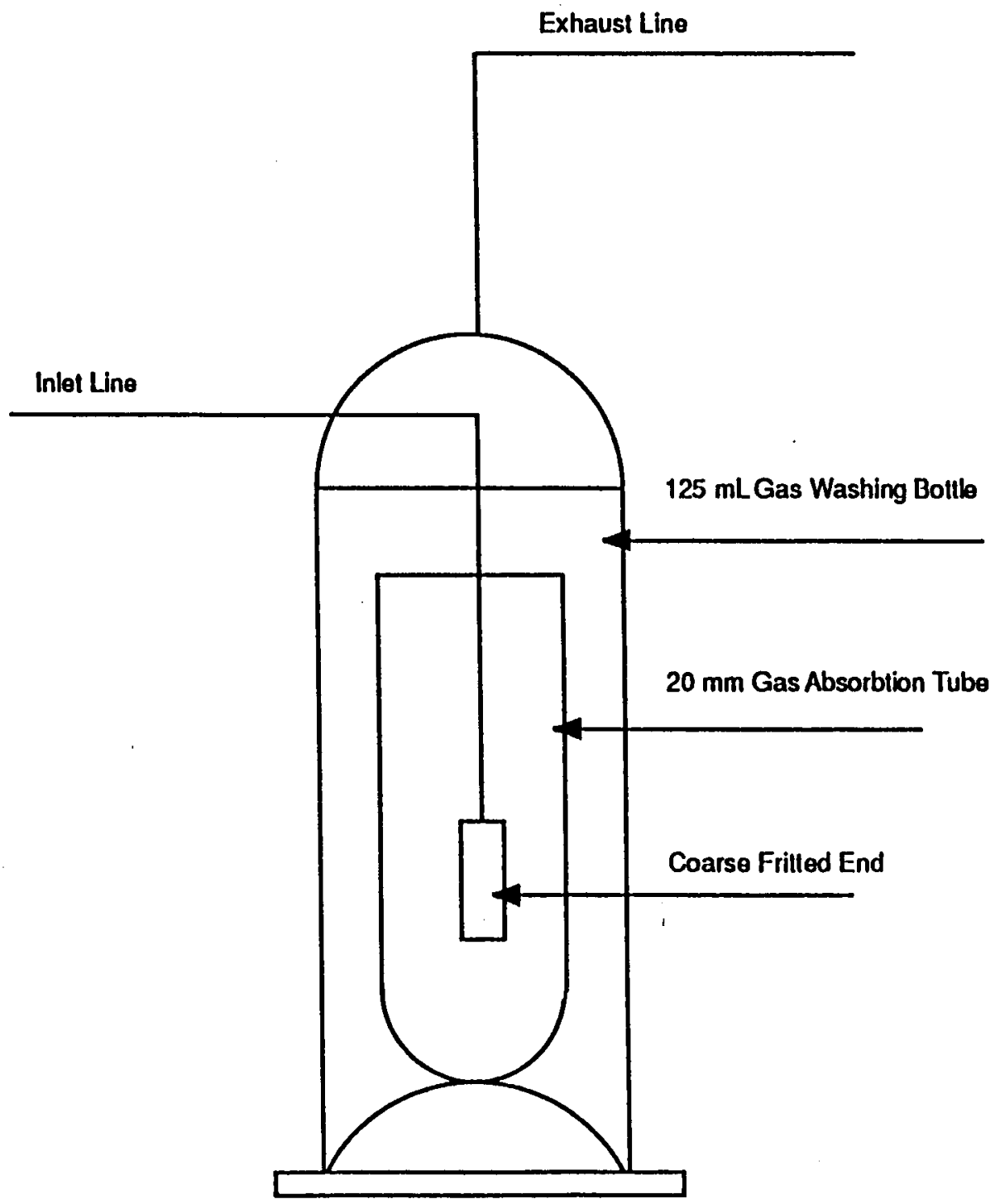

Figure 1. $125 \mathrm{~mL}$ Gas Washing Bollle 


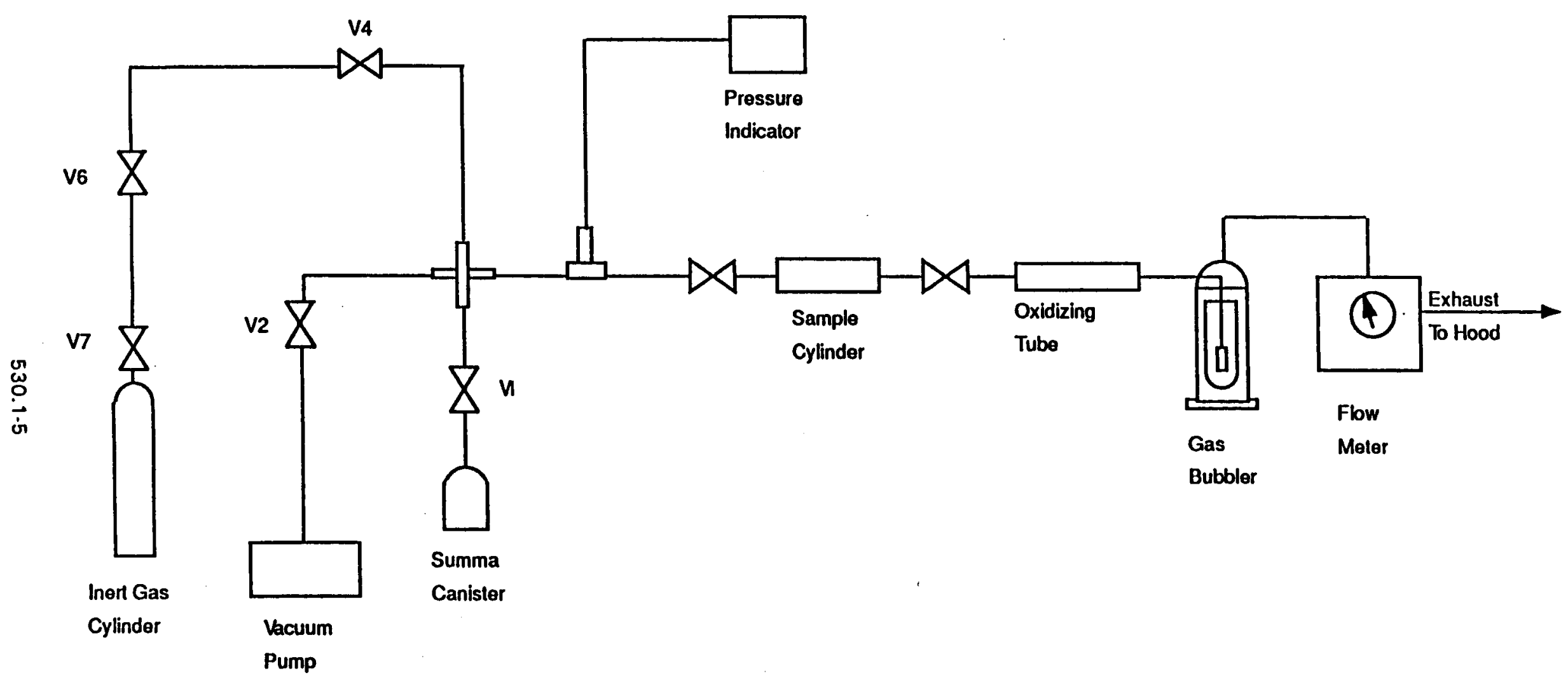

Figure 2. NOx Gas Analysis Equipment 
4.8 Inert Gas: High pressure inert gas cylinder.

4.9 Spectrophotometer or Colorimeter: A laboratory instrument suitable for measuring the intensity of the red-violet color at $550 \mathrm{~nm}$, with stoppered tubes or cuvettes. The wavelength bandwidth is not critical for this determination.

\subsection{REAGENTS}

5.1 Purity of Reagents: Reagent grade chemicals shall be used in all tests. Unless otherwise indicated it is intended that all reagents shall conform to the specifications of the Committee on Analytical Reagents of the American Chemical Society, where such specifications are available. Other grades may be used, provided it is first demonstrated that the reagent is of sufficiently high purity to permit its use without lessening the accuracy of the determination.

\subsection{Analysis: The following reagents are required for analysis:}

5.2.1 Water: Unless otherwise indicated, references to water shall be to deionized water in accordance with specifications in ASTM D1 193 Type II reagent water. Water must be nitrite-free.

5.2.2 Absorbing Reagent: Dissolve $5 \mathrm{~g}$ of anhydrous sulfanilic acid (or $5.5 \mathrm{~g}$ of the monohydratel in almost a liter of water containing $140 \mathrm{~mL}$ of glacial acetic acid. Gentle heating is permissible to speed up the process. To the cooled mixture, add $20 \mathrm{~mL}$ of the $0.1 \%$ stock solution of $\mathrm{N}-(1$-naphthyl)-ethylenediamine dihydrochloride and $10 \mathrm{~mL}$ acetone. Dilute to 1 liter. The solution will be stable for several months if kept well-stoppered in a brown bottle in the refrigerator. The absorbing reagent must be at room temperature before use. Avoid lengthy contact with air during both preparation and use, since absorption of nitrogen dioxide will discolor the reagent.

5.2.3 Chromic Acid Oxidizer: Dissolve $17 \mathrm{~g}$ of chromium trioxide in $100 \mathrm{~mL}$ water. Add $15 \mathrm{~g}$ of support medium (sand, molecular sieve, firebrick, etc.) slowly to prevent overheating. Let stand for approximately $30 \mathrm{~min}$. Pour off excess liquid and dry the support medium in an oven at $105^{\circ} \mathrm{C}$ until dry. The solid oxidizer should have a dull pink color. Let the solid oxidizer stand in the open air for 24 hours or until it turns bright vellow.

5.2.4 N(1-Naphthyl)-Ethylenediamine Dihydrochloride Stock Solution (0.1\%): Dissolve $0.1 \mathrm{~g}$ of the reagent in $100 \mathrm{~mL}$ of water. The solution will be stable for several months if kept well- 
stoppered in a brown bottle in the refrigerator. (Alternatively, weighed small amounts of the solid reagent may be stored.)

5.2.5 Sodium Nitrite $\left(\mathrm{NaNO}_{2}\right)$ Standard Solution $\left.10.0246 \mathrm{~g} / \mathrm{L}\right)$ : One milliliter of this working solution of $\mathrm{NaNO}_{2}$ produces a color equivalent to that of $20 \mu \mathrm{g}$ of $\mathrm{NO}_{2}$ in $1 \mathrm{~L} \mathrm{of} \mathrm{air} \mathrm{at} 760 \mathrm{~mm}$ $\mathrm{Hg}\left(101 \mathrm{kPa}\right.$ ) and $25^{\circ} \mathrm{C}$ (see 10.1 ). Prepare fresh just before use by diluting from a stock solution containing $2.46 \mathrm{~g} / \mathrm{L}$ of $\mathrm{NaNO}_{2}$ (calculated as $100 \%$ ). It is desirable to assay the solid reagent. The stock solution is stable for 90 days at room temperature, and for a year in a brown bottle under refrigeration.

5.3 Calibration Verification Standard: A calibration verification gas standard should be obtained and must be certified to $\pm 5 \%$. It must contain both $\mathrm{NO}$ and $\mathrm{NO}_{2}$ and be approximately midrange of the calibration curve.

\subsection{SAMPLE COLLECTION, PRESERVATION, AND HANDLING}

6.1 Samples will be collected using the sampling procedures described in the WIPP Experimental-Waste Characterization Program QAPP.

6.2 There are no method specific requirements for sample preservation or holding times prior to analysis. To facilitate timely reporting of the analysis data, sample holding times should not exceed 28 days.

6.3 All sample handling and chain-of-custody procedures described in the QAPP for the WIPP Experimental-Waste Characterization Program must be followed.

\subsection{PROCEDURE}

7.1 Canister Sampling Operation: Assemble apparatus for bubbling a known quantity of headspace gas from a SUMMA canister through an $\mathrm{NO}_{2}$ trapping solution. An example of such an apparatus is shown in Figure 2.

7.1.1 Add absorbing reagent to gas absorption tube $1=10 \mathrm{~mL}$ is required for 40 to 75 $\mathrm{mL}$ of gas sample). The exact quantity must be known and recorded. 
7.1.2 Evacuate the sample cylinder using the vacuum pump. Fill the sample cylinder with gas from the SUMMA canister. The exact quantity of gas (pressure, temperature, volume) must be known and recorded.

7.1.3 Flush gas from the sample cylinder through the oxidizer and bubbler using an inert gas. The flow should be such that quality assurance objectives (Table 1) can be met (between 130 and $180 \mathrm{~mL} / \mathrm{min}$ has been used).

7.1.4 Disconnect the gas bubbler from the system and let the absorbing solution stand for 15 minutes.

7.1.5 Measure the absorbance of the solution at $550 \mathrm{~nm}$ on the spectrophotometer.

7.1.6 After using the bubbler, rinse well with distilled water and dry. If the fritted tip is visibly discolored, clean according to the procedure in Section 4.1.2.

7.1.7 Remove the SUMMA canister from the apparatus unless a second analysis of the sample is necessary.

\subsection{Calibration:}

7.2.1 Standardization is based upon the empirical observation that $0.82 \mathrm{~mol}$ of $\mathrm{NaNO}_{2}$ produces the same color as $1 \mathrm{~mol}$ of $\mathrm{NO}_{2}$. One milliliter of the working standard solution contains 24.6 $\mu g$ of $\mathrm{NaNO}_{2}$. Since the molecular weight of $\mathrm{NaNO}_{2}$ is 69.0 this is equivalent to: $(24.6 / 69.0) \mathrm{X}$ $(46.0 / 0.82)=20 \mu \mathrm{g}$ of $\mathrm{NO}_{2}$.

7.2.2 Prepare a minimum of three standards and a blank by pipetting suitable volumes of standard solution into a series of $25-\mathrm{mL}$ volumetric flasks, and dilute to the marks with absorbing reagent. Mix, allow $15 \mathrm{~min}$ for complete color development, and read the absorbance. Standards must bracket the concentrations of the samples.

7.2.3 Plot the absorbances of the standards against micrograms of $\mathrm{NO}_{2}$ per milliliter of absorbing reagent. The plot follows Beer's law. Calculate the line of best fit using regression analysis by the method of least squares. Determine the value of the standardization factor, $K$, the number of micrograms of $\mathrm{NO}_{2}$ intercepted at an absorbance of exactly 1.0 (the reciprocal of the slope of the 
standardization curve). The correlation coefficient for the least squares fit of the data must be $\geq 0.995$.

7.2.4 Calibration checks must be run at the beginning and end of each analytical batch and at a frequency of $10 \%$ during the analytical sequence. The value from the continuing calibration check shall be compared to the corresponding value from the most recent valid calibration. If the RPD between the calibration curve value and the certified calibration check is less than $50 \%$, the $\mathrm{NO}_{\mathrm{x}}$ system shall be considered calibrated.

\subsection{Order of Analysis}

7.3.1 After measuring the calibration standards and assuring a linear calibration curve, the gas calibration verification standard and a gas laboratory control standard (LCS) should be measured. The samples should be measured following the LCS. Calibration checks are run at a frequency of $10 \%$ and at the end of the run.

\subsection{Analysis}

7.4.1 After canister sampling, development of the red-violet color is complete within 15 $\mathrm{min}$ at room temperatures. Transfer to a stoppered cuvette and read in a spectrophotometer at 550 $\mathrm{nm}$, using distilled water as a reference. The absorbance of the reagent blank must be subtracted from that of the sample.

7.4.2 Colors too dark to read may be quantitatively diluted with unexposed absorbing reagent. The resulting concentration is then corrected for the dilution factor.

\subsection{Calculations}

7.5.1 Compute the concentrations of total $\mathrm{NO}$ plus $\mathrm{NO}_{2}$ in the sample as follows:

Vol. $\% N O_{x}=\frac{\text { absorbance } \times K \times V_{a} \times R \times T}{M W_{N O_{2}} \times V \times P \times 10^{3}} \times 100$ 
where:

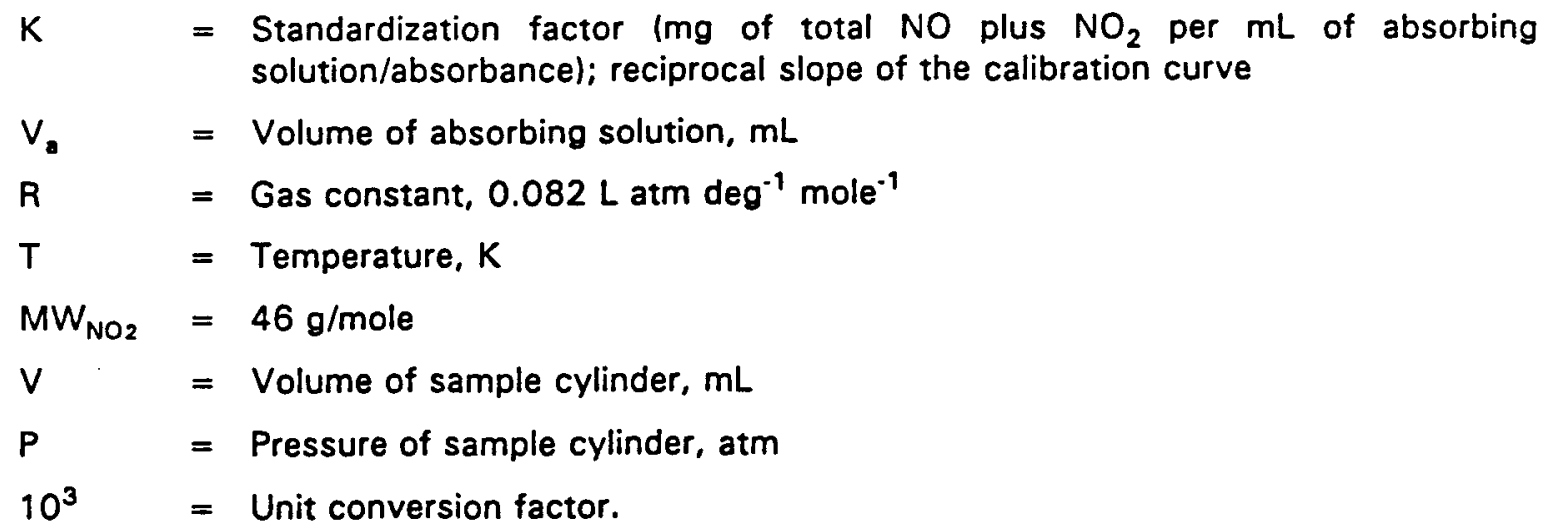

\subsection{Report}

7.6.1 Sample data must be reported according to the requirements specified by DOEMPO. The quantitative values shall be reported in units of volume percent (vol. \%) down to the MDL. Values for analytes analyzed for but not detected shall be reported as the MDL with the required "U" flag (i.e., if the MDL is 0.001 vol. \%, report $0.001 \mathrm{U}$ ). Analytical results, including MDL values used in reporting, must be corrected for dilution.

The numerical value assigned to the MDL for a given analyte shall include two significant figurs when the MDL is greater than or equal to $10 \%$ of the corresponding PRDL. If the MDL is less than $10 \%$ of the PRDL, its numerical value shall be reported with one significant figure. (Note: Under no circumstances is a value reported as all zeros acceptable.) Analytical results above the MDL shall be reported to a sufficient number of decimal places to include the least significant figure of the MDL, but to no more than three significant figures.

To illustrate implementation of these reporting requirements, consider the example of an analyte having a PRDL of 0.1 vol. \%. If the MDL for this analyte is 0.024 vol. \%, then a result of 0.09265 would be reported as 0.093 . If the MDL is 0.0008 vol. \%, then 0.09265 would be reported as 0.0926 .

\subsection{QUALITY CONTROL}

8.1 This section outlines the minimum necessary $Q C$ operations. The laboratory $O C$ frequency and acceptance criteria are summarized in Table 2. The following OC operations must be performed.

8.1.1 Calibrations Curves: A calibration curve is constructed from a minimum of three certified standards and a blank. The resulting calibration curve must yield a straight line with a 
Table 2. Scheduled $\mathrm{QC}$ and Calibration

\begin{tabular}{|c|c|c|c|}
\hline $\begin{array}{l}\text { Section } \\
\text { Number }\end{array}$ & Procedure & $\begin{array}{l}\text { Frequency of } \\
\text { OC Procedure }\end{array}$ & Acceptance Criteria \\
\hline 8.1 .1 & $\begin{array}{l}\text { Initial } \\
\text { calibration }\end{array}$ & Daily & $R>0.995$ \\
\hline 8.1 .2 & $\begin{array}{l}\text { Continuing } \\
\text { calibration }\end{array}$ & $\begin{array}{l}\text { At the beginning } \\
\text { and end of each } \\
\text { analytical batch } \\
\text { and at a frequency } \\
\text { of } 10 \%\end{array}$ & $\begin{array}{l}\% D \text { for all compounds } \\
\text { within } 50 \% \text { of initial } \\
\text { calibration }\end{array}$ \\
\hline 8.1 .5 & $\begin{array}{l}\text { Laboratory } \\
\text { control } \\
\text { sample }\end{array}$ & $\begin{array}{l}\text { One per analytical } \\
\text { batch }\end{array}$ & $\begin{array}{l} \pm 50 \% \text { recovery of certified } \\
\text { known value }\end{array}$ \\
\hline 8.1 .4 & $\begin{array}{l}\text { Laboratory } \\
\text { duplicate }\end{array}$ & $\begin{array}{l}\text { One per analytical } \\
\text { batch }\end{array}$ & $\begin{array}{l}\% D \text { for all NOx within } \\
20 \% \text { of the sample } \\
\text { results }\end{array}$ \\
\hline 8.1 .3 & $\begin{array}{l}\text { Laboratory } \\
\text { blank }\end{array}$ & $\begin{array}{l}\text { One per analytical } \\
\text { batch }\end{array}$ & $\begin{array}{l}\text { Target analytes less than } \\
\text { the PRDLs (Table } 1 \text { ) }\end{array}$ \\
\hline 8.2 & $\begin{array}{l}\text { Proficiency } \\
\text { testing }\end{array}$ & $\begin{array}{l}\text { Each laboratory, } \\
\text { every six months }\end{array}$ & \\
\hline
\end{tabular}


correlation coefficient $(R) \geq 0.995$. The low standard should be at five times the MDL or the PRDL (Table 1), whichever is higher, and the high standard will be such that it brackets the expected sample concentrations and remains in the linear range of the instrument.

8.1.2 Calibration Verification: Calibration verification is done prior to and after running samples and at a frequency of $10 \%$. This is done by measuring the quality check standard. This standard should be $\pm 50 \%$ of the accepted value. If it is not within these control limits, then the problem should be identified, the appropriate corrective action should be taken, the system should be recalibrated, and the samples should be reanalyzed back to the last good check standard.

\subsubsection{Blank Analysis}

8.1.3.1 Laboratory Blanks (LBs): SUMMA canisters will be filled with ultra-high purity nitrogen $(99.999 \%$ pure) in the laboratory using the sample preparation manifold. The same analytical procedure used to prepare the field samples for analysis will be used to prepare the laboratory blanks. Laboratory blank results will be acceptable if the concentration of the $\mathrm{NO}_{\mathrm{x}}$ is less than the PRDL. Remedial action must be taken for blanks which exceed the PRDL. Laboratory blanks will be analyzed daily during analytical operations.

\subsubsection{Duplicate Analysis}

8.1.4.1 Laboratory Duplicate: The laboratory shall analyze field samples in duplicate. Single canisters shall be analyzed in duplicate at a minimum frequency of one per analytical batch.

Single canisters analyzed in duplicate shall be used to assess laboratory precision. Laboratory duplicate results shall be considered acceptable for $N_{x}$ if the RPD is less than $\pm 20 \%$ for concentrations greater than five times the PRDL (Table 1), and the absolute difference is less than $\pm P R D L$ for concentrations less than five times the PRDL. Duplicates which do not meet these criteria should be flagged. It will be the responsibility of the laboratory QA Officer to monitor and document the results.

\subsubsection{Laboratory Control Sample (LCS): The LCS is used to monitor the laboratory's day-} to-day performance of routine analytical methods. An LCS consists of a standard gas mixture and must be from sources independent of those used for instrument calibration. The LCS is analyzed with field samples to provide evidence that the laboratory is performing the method within acceptable QC guidelines. The LCS must be run at least once per analytical batch. The value of this control standard 
should be $\pm 50 \%$ of its certified value. If it is not within the control limits, the problem should be identified and the appropriate corrective action should be taken. The system must be recalibrated and all of the samples in that batch must be reanalyzed.

8.2 All laboratories using these methods must demonstrate acceptable performance prior to the analysis of any samples. Demonstration of acceptable performance will be achieved by analyzing commercially available (Scott Specialty Gases or equivalent) gas standards. These standards shall contain $\mathrm{NO}$ and $\mathrm{NO}_{2}$ at concentrations appropriate (two to five times the PRDLs) to determine the parameters specified in Table 1. The analysis of four replicate samples must meet the criteria specified for precision, accuracy, and method detection limits in Table 1. Demonstration of acceptable method and analyst performance must be repeated at a minimum of every six months. It will be the responsibility of the QA Officer at each participating laboratory to monitor and document method and analyst performance. The laboratory $Q A$ Officer shall have responsibility to implement corrective actions when acceptable method performance is not met.

8.3 The laboratory will be required to analyze blind audit samples. These audit samples are part of the WIPP Performance Demonstration Program Plan. Details of this program are in the Performance Demonstration Program Plan.

\subsection{REFERENCES}

1. "D-3608 Standard Test Method for Nitrogen Oxides (Combined) in the Atmosphere by the Griess-Saltzman Reaction," Annual Book of ASTM Standards, Vol. 11.03.

2. "D-1193 Specification for Reagent Water," Annual Book of ASTM, Standards, Vol. 11.01.

3. "D-1356 Definitions of Terms Relating to Atmospheric Sampling and Analysis," Annual Book of ASTM Standards, Vol. 11.03.

4. "E-128 Test Method for Maximum Pore Diameter and Permeability of Rigid Porous Filters for Laboratory Use," Annual Book of ASTM Standards, Vol. 14.02. 


\subsection{INORGANICS}

As appropriate, future revisions of this Guidance Manual will include sample preparation procedures, clean-up procedures, and analytical procedures for the determination of metals, anions, and cations. 


\subsection{ASSAYS}

Future revisions of this Guidance Manual will provide guidance on quality control requirements for the determination of total alpha activity. 


\subsection{CHARACTERISTICS AND PROPERTIES}

This Guidance Manual contains the following procedure for the evaluation of headspace samples:

Procedure 810.1 provides a flame test procedure for the evaluation of the flammability of waste container headspace samples collected using SUMMA passivated canisters. 


\section{MODIFIED ASTM METHOD E 681-85 FOR THE EVALUATION OF THE FLAMMABILITY OF WASTE CONTAINER HEADSPACE USING SUMMA* PASSIVATED CANISTER SAMPLING}

\subsection{SCOPE AND APPLICATION}

1.1 This document describes a procedure for the use of a flame test apparatus (Figures 1 through 8) to evaluate the flammability of waste container headspace samples that contain upper confidence limit concentrations of flammable VOCs (Table 1) greater than $500 \mathrm{ppmv}$. This procedure is based on ASTM Method E 681-85, "Standard Method for Concentration Limits of Flammability of Chemicals" (ASTM, 1989). Waste container headspace sampling requirements are described in the QAPP for the WIPP Experimental-Waste Characterization Program; waste container headspace sampling procedures are described in this Guidance Manual (Method 110 .

1.2 Unlike ASTM Method E 681-85, this procedure is not designed to obtain a quantitative value for the upper explosive limit (UEL) or the lower explosive limit (LEL). Rather, this procedure specifies the conditions for the use of a flame test apparatus to qualitatively evaluate whether or not waste container headspace samples are flammable. The terms "upper explosive limit" and "lower explosive limit" are synonymous with upper flammability limit and lower flammability limit, respectively.

1.3 This procedure involves the use of hazardous materials, operations, and equipment. This procedure does not purport to address all of the safety problems associated with its use. It is the responsibility of each laboratory involved in flammability testing to consult and establish appropriate safety and health practices.

\subsection{SUMMARY OF PROCEDURE}

2.1 The flame test vessel and all connecting lines are evacuated. A SUMMA canister holding a headspace sample is attached to the flame test apparatus and the flame test vessel is pressurized with sample. After all safety precautions have been taken, a spark is delivered to the sample. If the sample propagates a flame upward and outward from the ignition source, it is flammable. Otherwise, it is not. 
Table 1. Flammable Volatile Organic Compounds" (VOCs) and their Lower Explosive Limit (LEL) Values ${ }^{\text {b.c }}$.

\begin{tabular}{|l|c|}
\hline \multicolumn{1}{|c|}{ VOCs } & LEL $(v / v \%)$ \\
\hline Acetone & 2.5 \\
\hline Benzene & 1.3 \\
\hline n-Butanol & 1.4 \\
\hline 2-Butanone & 1.4 \\
\hline Chlorobenzene & 1.3 \\
\hline Cyclohexane & 1.3 \\
\hline $1,1-$ Dichloroethane & 5.6 \\
\hline $1,2-$ Dichloroethane & 6.2 \\
\hline 1,1 -Dichloroethene & 6.5 \\
\hline cis-1,2-Dichloroethene & 5.6 \\
\hline Ethyl benzene & 1.0 \\
\hline Diethyl ether & 1.9 \\
\hline Methanol & 6.0 \\
\hline 4-Methyl-2-pentanone & 1.2 \\
\hline Toluene & 1.2 \\
\hline $1,2,4-$ Trimethylbenzene & 0.9 \\
\hline $1,3,5$-Trimethylbenzene & 1.4 \\
\hline o-Xylene & 1.0 \\
\hline m-Xylene & 1.1 \\
\hline p-Xylene & 1.1 \\
\hline
\end{tabular}

-VOCs identified as flammable by EPA for the No-Migration Determination. EPA determined flammability by assessing potential fire hazards under WIPP conditions on a compound by compound basis.

${ }^{b}$ The lower explosive limit is also refered to as the lower flammable limit (ASTM, 1989 and NFPA, 1986)

'Lower explosive limit values are reprinted from NFPA, 1986 and GPC, 1988. 
Revision: 0

Date: June 14, 1991

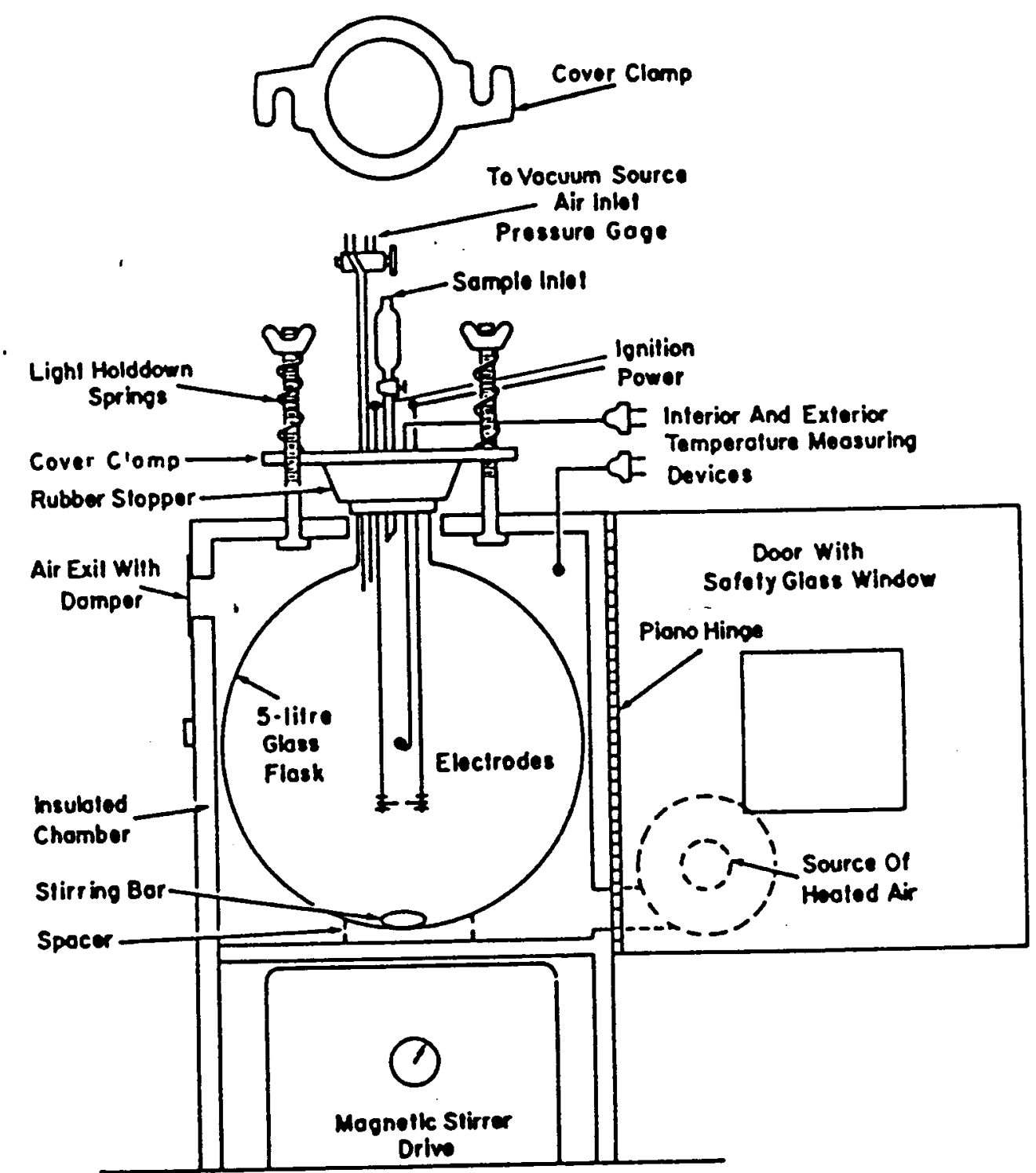

Figure 1. Schematic Diagram I of Flame Test Apparatus as Specified in ASTM Method E 681-85 


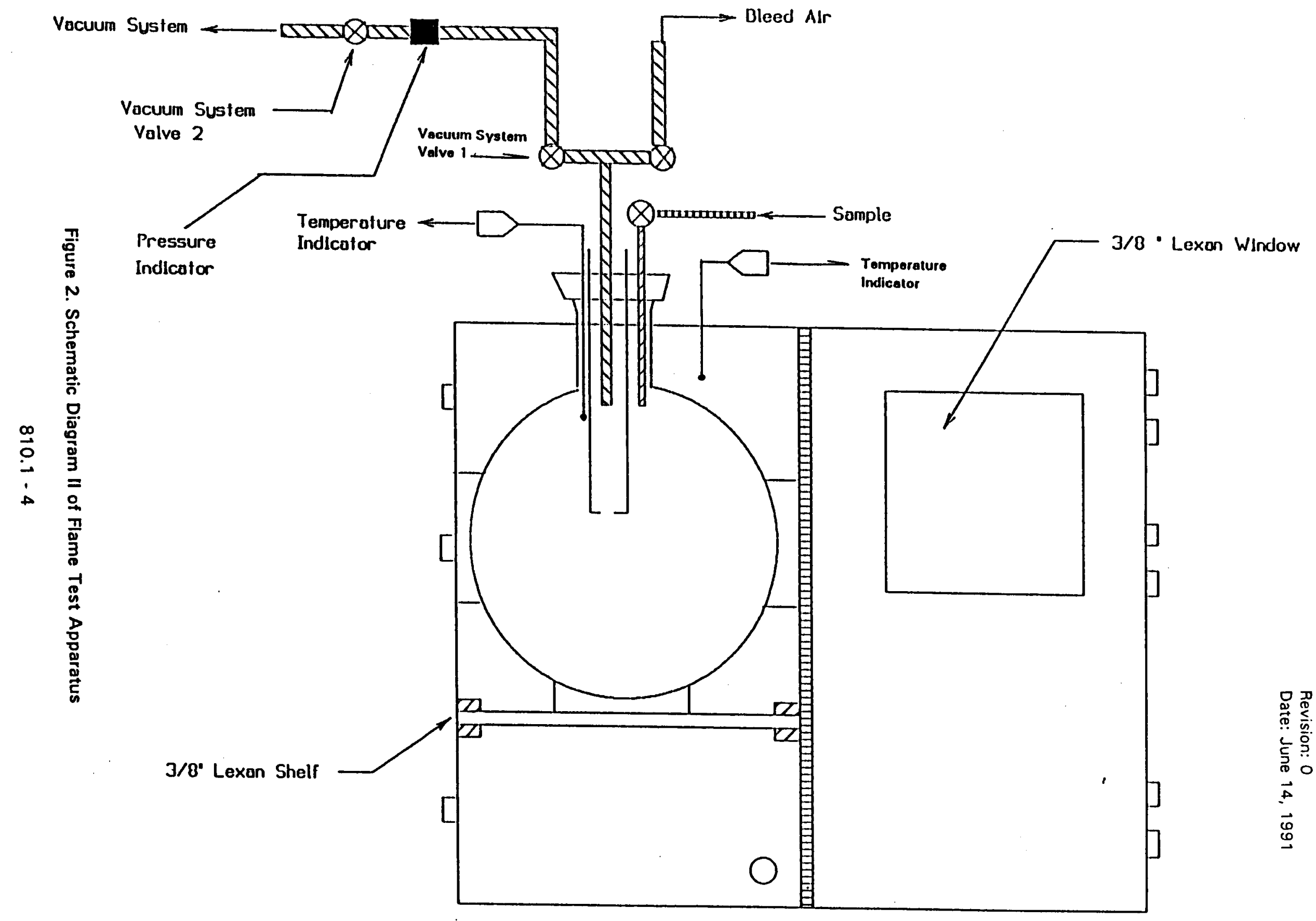




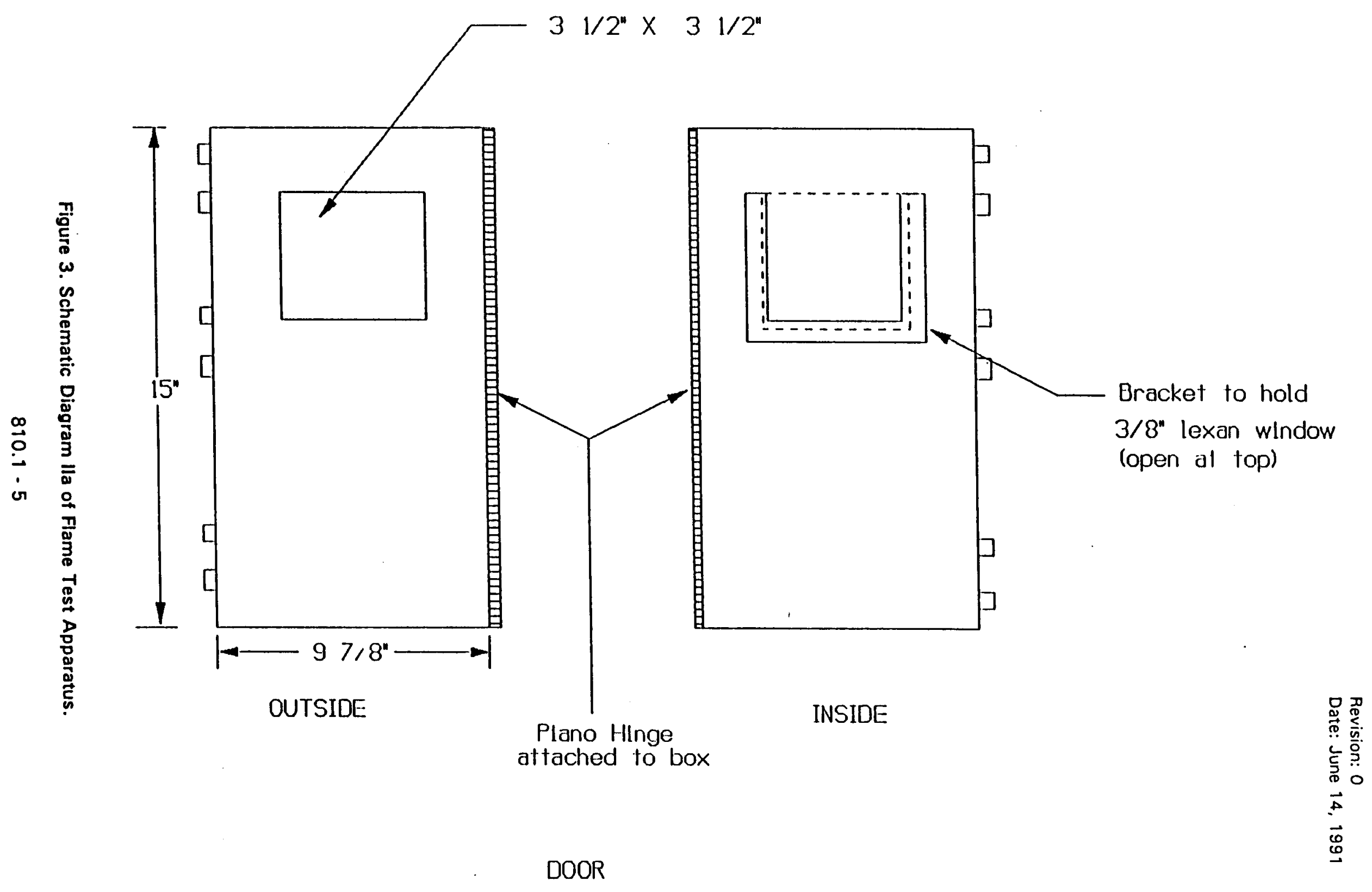




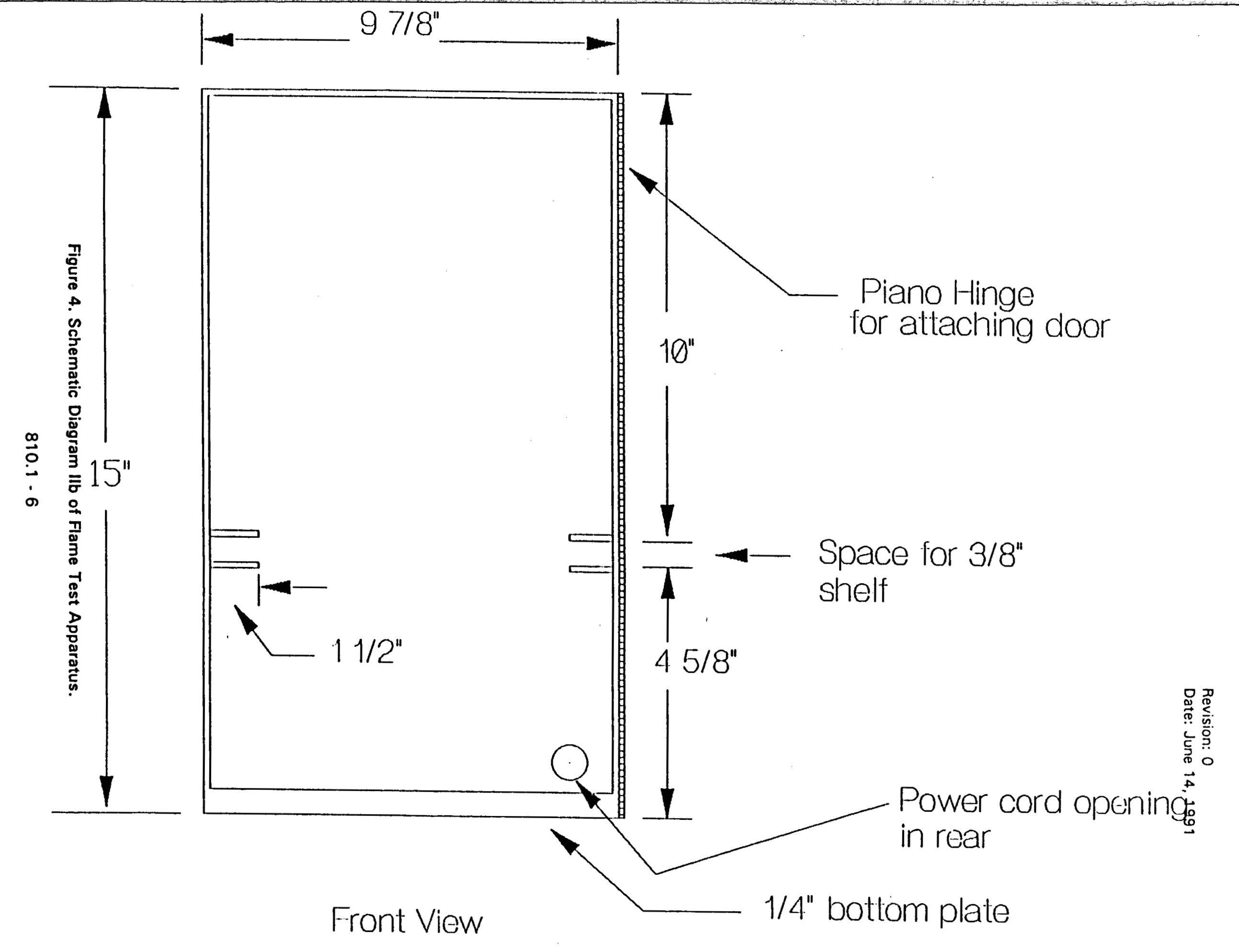




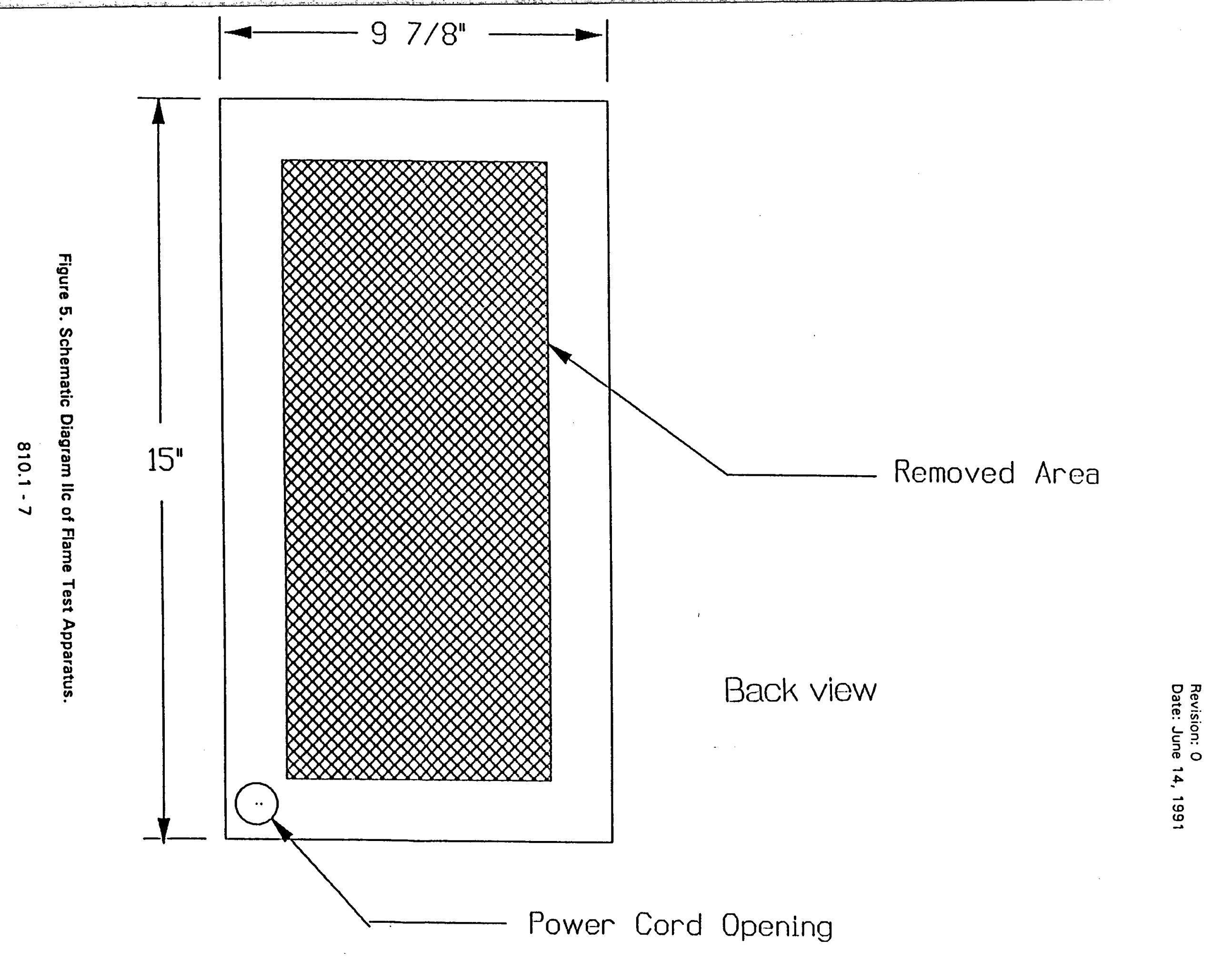




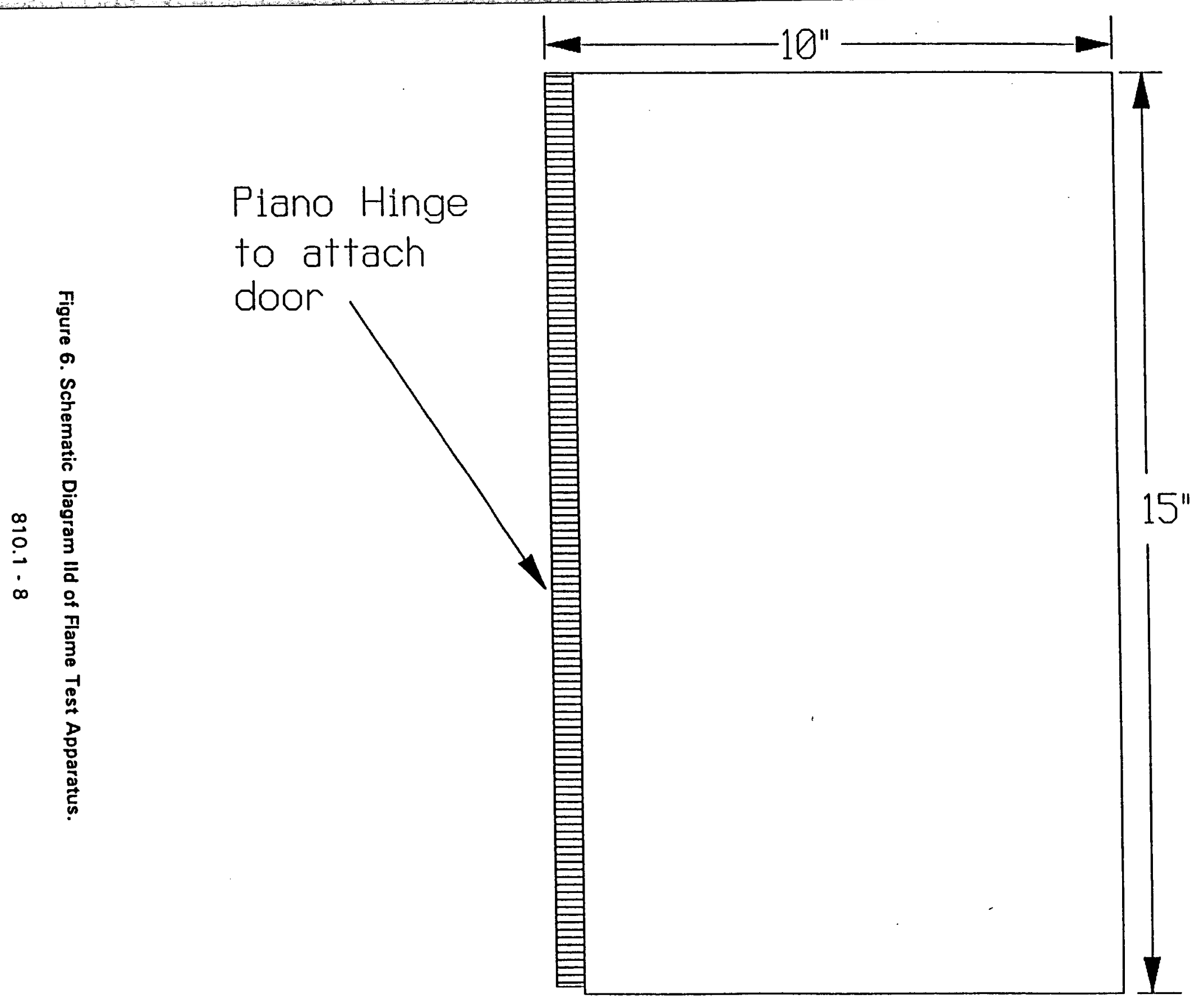

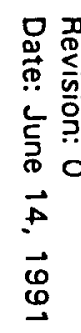

Right side 


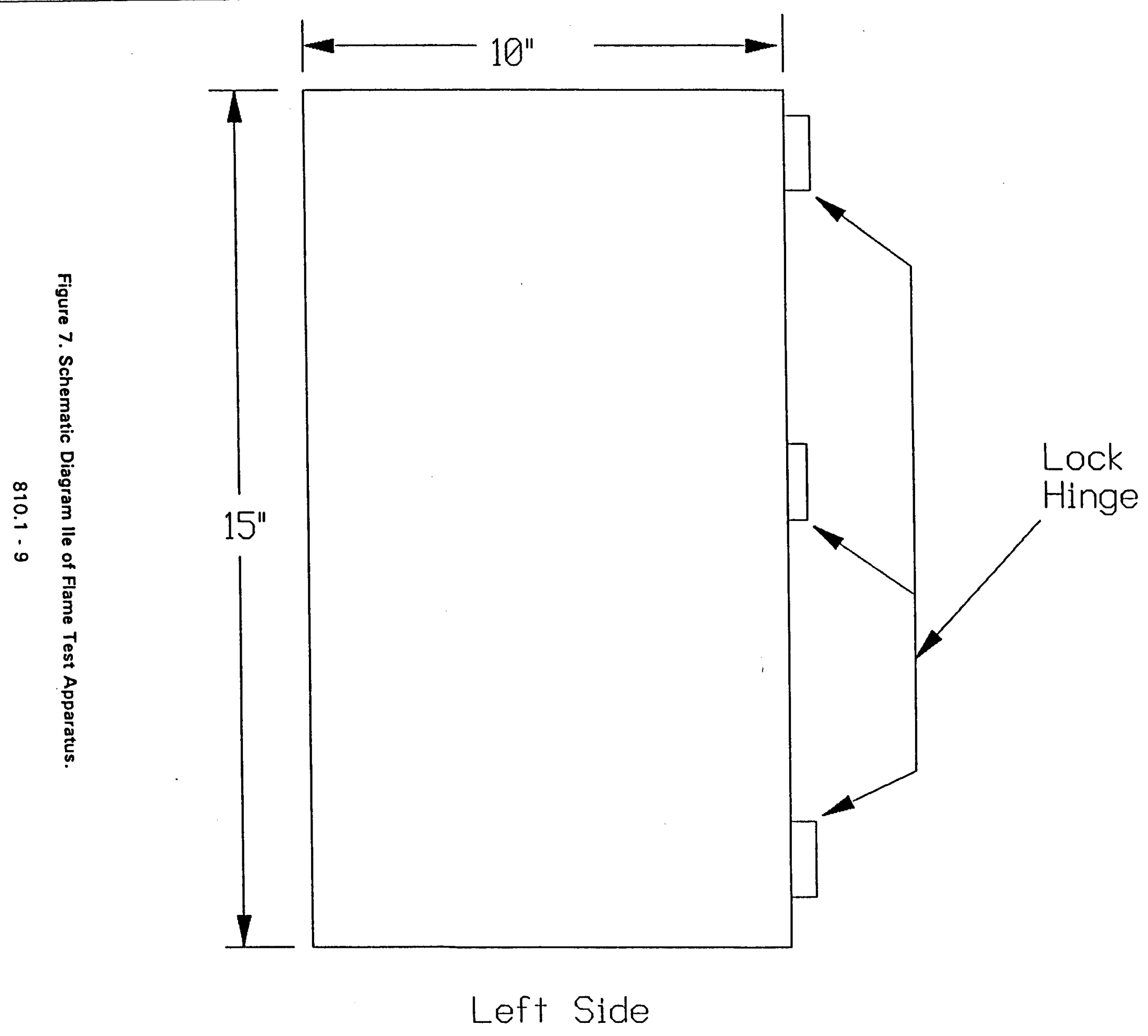

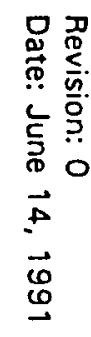




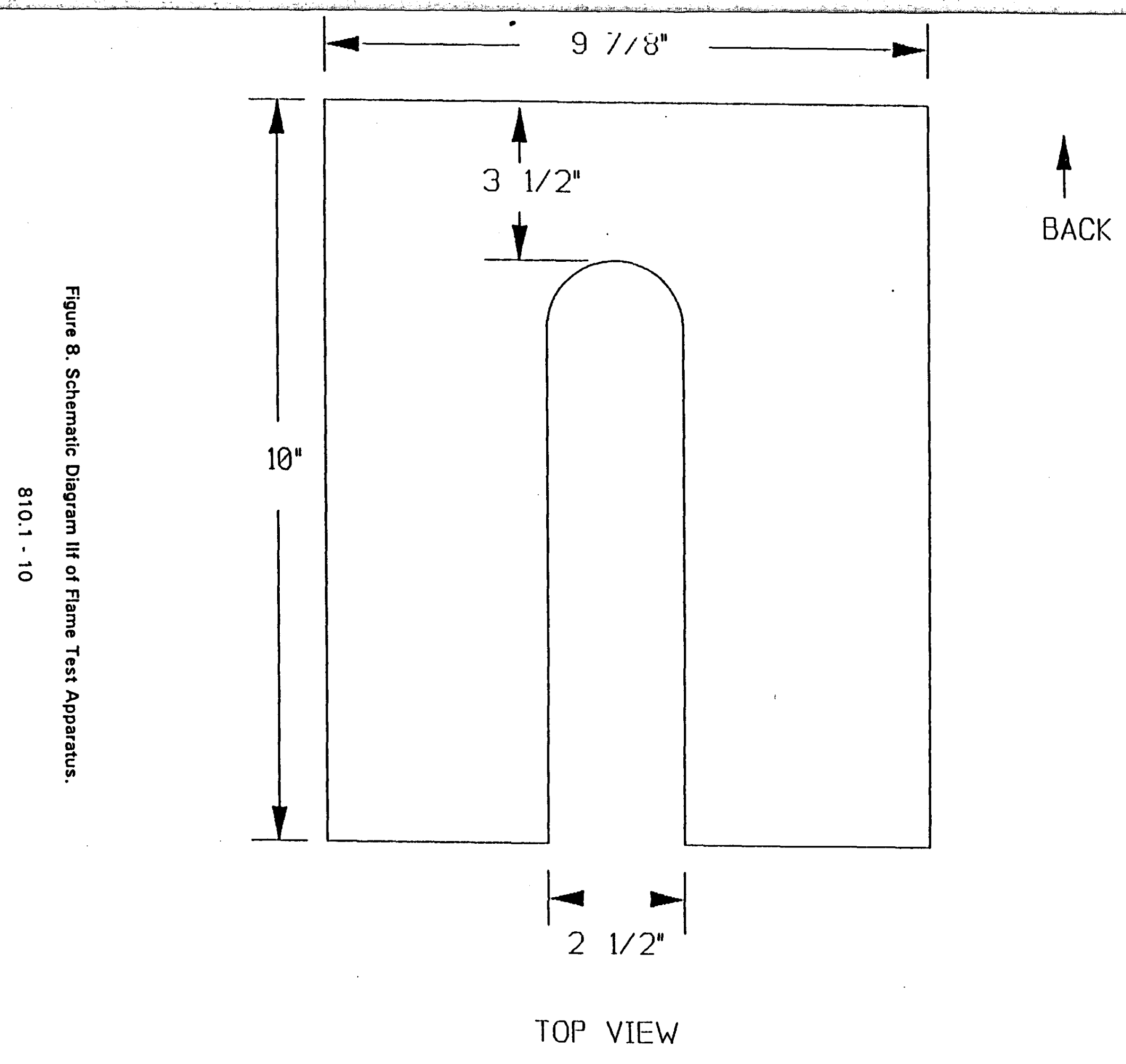




\subsection{INTERFERENCES}

3.1 Flame quenching effects of the test vessel walls are mitigated by the diameter of the flame test vessel used. The $5-L$ flame test vessel used in this procedure has a sufficient diameter to eliminate the effects of flame quenching for most materials.

3.2 The slightest vacuum leak can invalidate the test results. Prior to each flammability determination, the flame test apparatus and all connecting lines must pass a vacuum inspection.

3.3 The neat compounds used for generating initial and continuing calibration standards may condense before reaching the test vessel. Applying a gentle heat source to the connections above the test vessel during pressurization will ensure quantitative transfer of all neat compound material to the test vessel.

3.4 Normal variations in atmospheric pressure do not appreciably affect flammability limits. However, the effect of large pressure variations is neither simple nor uniform but is specific to each flammable mixture (Coward and Jones, 1952). The change in the LEL for the first few hundred millimeters of mercury fall in pressure below atmospheric is imperceptible; thereafter the range of flammability is narrowed by raising the LEL and lowering the UEL. Flammability testing must be conducted at a pressure not less than 400 torr and not more than 760 torr. All calibrations and subsequent field sample evaluations must be performed at ambient temperature and the same final pressure.

\subsection{APPARATUS AND MATERIALS}

\subsection{Flame Test Apparatus Structural Components}

4.1.1 Stainless Steel Enclosure: An enclosure made entirely of welded stainless steel with overall dimensions sufficient to accommodate the flame test vessel and all necessary connections (Figures 2 through 8 ). Corners should be turned for structural integrity.

4.1.2 Flame Test Vessel: A 5-L, borosilicate glass, long-neck boiling flask (\# 601000-1045, Kontes Chemistry and Life Science Products, Vineland, NJ or equivalent).

4.1.3 Shelf: A shelf inserted inside the stainless steel enclosure to hold the test vessel. 
4.1.4 Test Vessel Cover: A \#14 rubber stopper to rest on top of the test vessel not inside the neck. The stopper should be drilled with the appropriate holes to accommodate electrodes, temperature sensors, sample inlet tubing, etc.

\subsection{Flame Test Apparatus Electrical Components}

4.2.1 Temperature Measuring Device (internall): A thermocouple, resistance thermometer, or thermistor capable of measuring the temperature inside the test vessel to $\pm 1^{\circ} \mathrm{C}$ under ambient conditions (\#PRX-NP-100-E-7 and \#DP703RTD, Omega Engineering Inc., Stanford, CT or equivalents).

4.2.2 Temperature Measuring Device (externall: A thermocouple, resistance thermometer, or thermistor capable of measuring the temperature outside the test vessel but within the stainless steel enclosure to $\pm 4^{\circ} \mathrm{C}$ under ambient conditions (\#PRX-AP-100-E-8 and \#DP703RTD, Omega Engineering Inc., Stanford, CT or equivalents).

4.2.3 Magnetic Stirrer and Stirring Bar: A magnetic stirrer capable of propelling a 2 1/2-in., egg-shaped stirring bar within the test vessel (\#11-500-4S, Fisher Scientific, Pittsburgh, PA or equivalent).

4.2.4 Heat Gun: A heat source capable of providing steady heat to the connections associated with the introduction of standards into the test vessel (\#09-201-10, Fisher Scientific, Pittsburgh, PA or equivalent).

4.2.5 Ignition Source: A power system able to deliver approximately $30 \mathrm{~mA}$ at $15 \mathrm{KV}$ (Gaslighter Mark 6 Model 12E, Channel Products, Chesterland, $\mathrm{OH}$ or equivalent).

4.2.6 Electrode Rods: Stainless steel rods $1 / 8$ to 3/16-in. diameter approximately 12 1/2in. long. The upper ends are threaded for connection to the high voltage source; the lower ends bent to form a spark gap. The rods should be spaced at least 1 1/4-in. apart, and form a spark gap of about $1 / 4$-in (stainless steel welding rods or equivalent).

\subsection{Flame Test Apparatus Vacuum Components}

\subsubsection{Pressure-Measuring System: A pressure-measuring system capable of indicating} pressures in the range from 5 to 760 torr with an accuracy of $\pm 0.2 \%$ (\#PX-425-015AV plus \#DP280P10, Omega Engineering Inc., Stanford, CT or equivalents). 
4.3.2 Vacuum Source: A vacuum source capable of reducing the pressure within the test vessel and all connecting lines to less than 30 torr (\#DD-20, Fisher Scientific, Pittsburgh, PA or equivalent). If a vacuum pump is used, an oil trap should be placed in-line between the vacuum pump and the test vessel to prevent back-flow of vacuum pump oil into the test vessel.

\subsection{REAGENTS}

5.1 Calibration Standards: Initial calibration standards are $8.0 \%(\mathrm{v} / \mathrm{v})$ methanol, $15 \%(\mathrm{v} / \mathrm{v})$ methanol, and $1.0 \%(\mathrm{v} / \mathrm{v})$ diethyl ether. The $8.0 \%(\mathrm{v} / \mathrm{v})$ methanol is used for continuing calibration.

5.1.1 Calibration standards are prepared by volatilizing and then air-diluting neat compounds in the flame test vessel. Liquids used for standards must be reagent grade or better. Calibrations and subsequent sample evaluations are performed at ambient temperature and a final test vessel pressure between 400 and 760 torr. The final test vessel pressure used for calibrations must be used for sample evaluations. The volume of liquid standard required to produce a calibration concentration in the test vessel is calculated as,

$$
\begin{gathered}
\mu L=\frac{(M W)(P)(V \%)(1000)}{(d)(T)(k)} \\
k=\frac{\left(V_{0}\right)\left(P_{0}\right)(100)}{(V)\left(T_{0}\right)}
\end{gathered}
$$

where:

$\mu \mathrm{L} \quad=$ volume of liquid standard to be injected into the test vessel

$\mathrm{MW}=$ molecular weight of liquid standard, $\mathrm{g} \mathrm{mol}^{-1}$

$P \quad=$ final test vessel pressure, $\mathrm{mm} \mathrm{Hg}$

$v \%=$ concentration of volatilized standard in test vessel, $v / v \%$

$d=$ density of liquid standard at $20^{\circ} \mathrm{C}, \mathrm{g} \mathrm{cm}^{-3}$

$\mathrm{T} \quad=$ temperature within test vessel, $K$

$V_{0}=$ standard molar volume, $22.4 \mathrm{~L}$

$P_{0}=$ standard pressure, $760 \mathrm{~mm} \mathrm{Hg}$

$V \quad=$ volume of test vessel, $\mathrm{L}$

$T_{0}=$ standard temperature, $273 \mathrm{~K}$. 
For example, the molecular weight of methanol is $32 \mathrm{~g} / \mathrm{mole}$ and at $20^{\circ} \mathrm{C}$ the density of methanol is $0.7913 \mathrm{~g} / \mathrm{cm}^{3}$. In a 5.000 liter test vessel at $25^{\circ} \mathrm{C}$ and $400 \mathrm{torr}$, the required volume for the $8.0 \%$ $(v / v)$ methanol calibration standard is $348 \mu \mathrm{L}$.

\subsection{SAMPLE COLLECTION, PRESERVATION AND HANDLING}

6.1 Samples must be collected using the sampling procedures described in the WIPP ExperimentalWaste Characterization Program QAPP.

6.2 Sample canisters must be stored at room temperature. Sample holding times cannot exceed 28 days.

6.3 All sample handling and chain-of-custody procedures described in the QAPP for the WIPP Experimental-Waste Characterization Program must be followed.

\subsection{PROCEDURE}

\subsection{Equipment Calibration}

7.1.1 The test vessel volume must be accurately determined. Weigh the clean, dry test vessel to $\pm 1 \mathrm{~g}$. Fill the flask with distilled water and insert all components allowing the excess water to overflow. Remove the components, dry the outside of the vessel, and reweigh. Record the difference in grams as the net volume of the vessel in cubic centimeters. The measurement of volume will need to be performed once on every $5 \mathrm{~L}$ flask used as a test vessel. Slight errors associated with water density differences are beyond the accuracy of this procedure.

7.1.2 Calibrate temperature-measuring devices and the pressure-measuring system in accordance with manufacturer's specifications.

\subsection{Initial Calibration}

7.2.1 Disconnect any canisters from the apparatus. Examine the structural integrity of the test vessel and ensure that no residual material, either from previous calibrations or from the testing of samples, is present. 
7.2.2 Open all valves and turn on the vacuum pump. Flush the apparatus and all connecting lines with air for about 30 seconds. Turn off the vacuum pump and close all valves.

7.2.3 Ensure that a spark is generated between the electrodes by activating the ignition source.

7.2.4 Attach a septum to the end of the bleed-air line. With the sample valve and the bleedair valve closed, open both vacuum system valves. Evacuate the test vessel to a pressure of 30 torr. Close vacuum system valve 2 and shut off the vacuum pump.

7.2.5 Leak-check the apparatus by monitoring the pressure within the apparatus for five minutes. If during the five minute period the pressure fluctuates by more than 1.5 torr, corrective action must be taken. Calibration cannot continue until the apparatus is capable of maintaining a vacuum of $30.0 \pm 1.5$ torr for five minutes.

7.2.6 Using Equations (1) and (2), calculate the volume of liquid standard to be injected into the test vessel.

7.2.7 Take up the calculated volume of liquid standard with an appropriately-sized microliter syringe, and inject the liquid standard into the bleed-air line through the septum.

7.2.8 Turn on the magnetic stirrer and allow the apparatus to equilibrate at ambient temperature. Record the temperature within the test vessel. Slowly open the bleed-air line valve to allow the liquid sample to be drawn into the test vessel. Insure the quantitative transfer of the liquid sample through the bleed-air line into the test vessel by gently heating all bleed-air line connections.

7.2.9 As the pressure stabilizes, remove the septum on the bleed-air line and bring the pressure within the test vessel to a pressure between 400 and 760 torr. Close the bleed-air valve and record the final pressure within the apparatus. Close vacuum system valve 1 .

7.2.10 Allow the apparatus to equilibrate for five minutes.

7.2.11 After equilibration, note the temperature within the test vessel and record the temperature within the enclosure. An elevated temperature reading from within the test vessel may result from heating the bleed-air line (Section 7.2.8). 
7.2.12 Turn off both internal and external temperature-measurement devices and the pressure-measuring device. Confirm that vacuum system valves 1 and 2 are closed and the vacuum pump is turned off.

7.2.13 Take appropriate safety precautions. Darken the viewing area. Activate the ignition source.

7.2.14 If the mixture is flammable, a flame is generated with enough explosive force to propagate to the test vessel walls and lift the rubber stopper about 10 millimeters off the top of the flask.

7.2.15 Calibration is successful if the $8.0 \mathrm{v} / \mathrm{v} \%$ methanol and the $15 \mathrm{v} / \mathrm{v} \%$ methanol standards are evaluated as flammable, and the $1.0 \mathrm{v} / \mathrm{v} \%$ diethyl ether standard is evaluated as nonflammable.

7.2.16 An initial calibration must be performed at least once per 20 field samples.

\subsection{Continuing Calibration}

7.3.1 Continuing calibration must be performed at the beginning of each working day prior to the evaluation of samples.

7.3.2 Follow the procedures described in Sections 7.2.1 through 7.2.13 using an $8.0 \mathrm{v} / \mathrm{v} \%$ methanol standard.

7.3.3 Continuing calibration is achieved when the $8.0 \mathrm{v} / \mathrm{v} \%$ methanol standard is evaluated as flammable.

\subsection{Sample Evaluation}

7.4.1 Disconnect any canisters from the apparatus. Examine the structural integrity of the test vessel and ensure that no residual material, either from previous calibrations or from the testing of samples, is present.

7.4.2 Open all valves and turn on the vacuum pump. Flush the apparatus and all connecting lines with air for about 30 seconds. Turn off the vacuum pump and close all valves. 
7.4.3 Ensure that a spark is generated between the electrodes by activating the ignition source.

7.4.4 Attach a canister containing a headspace sample to the sample line. With the canister valve and the bleed-air valve closed, open the sample valve. Open both vacuum system valves. Turn on the vacuum pump and evacuate the test vessel to a pressure of 30 torr. Close vacuum system valve 2 and turn off the vacuum pump.

7.4.5 Leak-check the apparatus by monitoring the pressure within the apparatus for five minutes. If during the five minute period the pressure fluctuates by more than 1.5 torr, corrective action must be taken. Sample evaluation cannot continue until the apparatus is capable of maintaining a vacuum of $30 \pm 1.5$ torr for five minutes.

7.4.6 Slowly open the sample valve and allow the pressure differential between the test vessel and canister to fill the test vessel.

7.4.7 Continue filling the test vessel with sample until the pressure within the test vessel is identical to the pressure at which initial and continuing calibrations were performed. Close the sample valve and record the pressure. Close vacuum system valve 1.

7.4.8 Close the canister valve and remove the canister.

7.4.9 Allow the apparatus to equilibrate at ambient temperature for five minutes.

7.4.10 After equilibration, record the temperature within the test vessel and the temperature within the enclosure.

7.4.11 Turn off both internal and external temperature-measurement devices and pressuremeasuring device. Confirm that vacuum valves 1 and 2 are closed and the vacuum pump is turned off.

7.4.12 Take appropriate safety precautions. Darken the viewing area. Activate the ignition source. 
7.4.13 If the sample is flammable, a flame is generated with enough explosive force to propagate to the test vessel walls and lift the rubber stopper about 10 millimeters off the top of the flask.

7.4.14 Record the results.

\subsection{QUALITY CONTROL}

8.1 Each laboratory that uses this procedure is required to operate a formal quality control program. Each laboratory must have standard operating procedures in place that document and describe the activities involved in utilizing this procedure.

8.2 The laboratory must retain records to document the quality of data generated. Figures 9 and 10 specify the information required to document data quality.

8.3 Quality control practices will include, but are not limited to, operational checks, operator training, duplicates, and blanks.

8.3.1 Operational checks include examination of the structural integrity of the test vessel, examination of the cleanliness of the test vessel, inspection of the ignition source power output, and verification of the pressure stability of the test vessel and all connecting lines.

8.3.2 Operators must successfully complete all applicable laboratory training program requirements. The samples of an analytical batch must be analyzed by the same operator who performed the initial and continuing calibrations associated with that analytical batch.

8.3.3 All duplicates will be evaluated in the same way as field samples. Laboratory duplicates will be evaluated at a minimum frequency of one per twenty field samples. All field duplicates will be evaluated.

8.3.4 The $1.0 \mathrm{v} / \mathrm{v} \%$ ether serves as a functional blank and none other is required.

8.4 All laboratories evaluating headspace samples for flammability must qualify the accuracy of their flammability testing apparatus. A flammability testing apparatus is considered qualified when it accurately evaluates the flammability of $8.0 \%(\mathrm{v} / \mathrm{v})$ methanol, $15 \%(\mathrm{v} / \mathrm{v})$ methanol, and $1.0 \%(\mathrm{v} / \mathrm{v})$ diethyl ether. Qualification must occur prior to the evaluation of field samples and annually thereafter. 
Revision: 0

Date: June 14, 1991

FLAMMABILITY TESTING DATA SHEET COVER PAGE

Analytical Batch No.:

Laboratory Name:

Initial Calibration Date:

Laboratory Code:

Operator Signature:

Initial Calibration Data:

\begin{tabular}{|c|c|c|c|c|c|c|}
\hline $\begin{array}{c}\text { Standard } \\
\text { (v/v\%) }\end{array}$ & $\begin{array}{l}\text { Vol. } \\
\text { Liquid, } \\
\text { Injected } \\
\text { (wL) }\end{array}$ & $\begin{array}{l}\text { Internal } \\
\text { Vessel } \\
\text { Temperature } \\
\left.{ }^{\circ} \mathrm{C}\right)\end{array}$ & $\begin{array}{l}\text { External } \\
\text { Vessel } \\
\text { Temperature } \\
\left.{ }^{\circ} \mathrm{C}\right)\end{array}$ & $\begin{array}{l}\text { lnitial } \\
\text { Vessel } \\
\text { Pressure } \\
\text { (torn) }\end{array}$ & $\begin{array}{l}\text { Final, } \\
\text { Vessel } \\
\text { Pressure } \\
\text { (torr) }\end{array}$ & $\begin{array}{l}\text { Flammability } \\
\text { Evaluation } \\
\text { (yes/no) }\end{array}$ \\
\hline $8.0 \%$ Methanol & & & & & & \\
\hline $15 \%$ Methanol & & & & & & \\
\hline $1.0 \%$ Diethyl ether & & & & & & \\
\hline
\end{tabular}

Samples Analyzed:

\begin{tabular}{|l|l|l|l|l|}
\hline Field Sample & Laboratory & & Field Sample & Laboratory \\
\hline & Sample & & Number & Sample \\
\hline & Number & & Number \\
\hline & & & & \\
\hline & & & & \\
\hline & & & & \\
\hline & & & & \\
\hline & & & & \\
\hline
\end{tabular}

Authorization to Release Data:

Date:

Title:

Figure 9. Sample Cover Page.

EXAMPLE ONLY 
Revision: 0

Date: June 14, 1991

FLAMMABILITY TESTING DATA SHEET

Analytical Batch No.:

Initial Calibration Date:

Operator Signature:

Continuing Calibration Data:

\begin{tabular}{|c|c|c|c|c|c|c|c|}
\hline $\begin{array}{l}\text { Continuing } \\
\text { Calibration } \\
\text { Date }\end{array}$ & $\begin{array}{l}\text { Vol, MeOH } \\
\text { Injected } \\
\text { lul })_{\text {p }}\end{array}$ & $\begin{array}{l}\text { Internal } \\
\text { Vessel } \\
\text { Temperature } \\
l^{\circ} \mathrm{Cl}\end{array}$ & $\begin{array}{l}\text { External } \\
\text { Vessel } \\
\text { Temperaturo } \\
\text { ( }{ }^{\circ} \mathrm{C} \text { ) }\end{array}$ & $\begin{array}{l}\text { Initial } \\
\text { Vessel } \\
\text { Pressure } \\
\text { (torr) }\end{array}$ & $\begin{array}{l}\text { Final } \\
\text { Vessel } \\
\text { Pressure } \\
\text { (torr) }\end{array}$ & $\begin{array}{l}\text { MeOH Conc. } \\
\text { in Vessel } \\
(v / v \%)\end{array}$ & $\begin{array}{l}\text { Flammability } \\
\text { Evaluation } \\
\text { (yes/no) }\end{array}$ \\
\hline & & & & & & & \\
\hline & & & & & & & \\
\hline & & & & & & & . \\
\hline & & & & & & & \\
\hline & & & & & & & \\
\hline
\end{tabular}

Laboratory Name:

Laboratory Code:

Sample Data:

\begin{tabular}{|c|c|c|c|c|c|c|c|}
\hline $\begin{array}{c}\text { Leboratory } \\
\text { Sample } \\
\text { No. }\end{array}$ & $\begin{array}{l}\text { Date } \\
\text { Sample } \\
\text { Received }\end{array}$ & $\begin{array}{l}\text { Date } \\
\text { Sample } \\
\text { Analyzed }\end{array}$ & $\begin{array}{l}\text { Internal } \\
\text { Vessel } \\
\text { Tempersture } \\
\left({ }^{\circ} \mathrm{C}\right)\end{array}$ & $\begin{array}{l}\text { External } \\
\text { Vessel } \\
\text { Temperature } \\
\left.{ }^{\circ} \mathrm{C}\right)\end{array}$ & $\begin{array}{l}\text { Initial } \\
\text { Vessel } \\
\text { Pressure } \\
\text { (torr) }\end{array}$ & $\begin{array}{l}\text { Final } \\
\text { Vessel } \\
\text { Pressure } \\
\text { (torr) }\end{array}$ & $\begin{array}{l}\text { Flammability } \\
\text { Evaluation } \\
\text { (yes/no) }\end{array}$ \\
\hline & & & & & & & \\
\hline & & & & & & & \\
\hline & & & & & & & \\
\hline & & & & & & & \\
\hline & & & & & & & \\
\hline & & & & & & & \\
\hline & & & & & & & \\
\hline & & & & & & & \\
\hline & & & & & & & \\
\hline & & & & & & & \\
\hline & & & & & & & \\
\hline
\end{tabular}

Figure 10. Sample Data Sheet. EXAMPLE ONLY 


\subsection{REFERENCES}

1. American Society for Testing and Materials, 1989, Method E 681-85, "Standard Method for Concentration Limits of Flammability of Chemicals", 1916 Race St., Philadelphia, Pennsylvania ASTM - See American Society for Testing and Materials

2. Coward, HF and Jones, GW, 1952, Limits of Flammability of Gases and Vapors, NTIS No. AD-701 575, US Dept. of Commerce, Springfield, Virginia

3. Channel Products, Chesterland, OH 44026

4. Fisher Scientific, Pittsburgh, PA (412) $562-8300$

5. Genium Publishing Corporation, 1988, Material Safety Data Sheet from Genium's Reference Collection, Genium Publishing Corporation, Schnectady, New York

6. GPC - See Genium Publishing Corporation

7. Kontes Chemistry and Life Sciences Products, Vineland, NJ (609) 692-8500

8. National Fire Protection Association, 1986, Fire Protection Guide on Hazardous Materials, 9th. ed., Batterymarch Park, Quincy, Massachusetts

9. NFPA - See National Fire Protection Association

10. Omega Engineering Inc., Stanford, CT (203) 359-1660 